IN VITRO AND IN VIVO CHARACTERISATION OF P. AERUGINOSA OXIDOREDUCTASE ENZYMES IN PATHOGENESIS AND THERAPY

BY

\title{
LAURA KAY GREEN
}

A thesis

Submitted to the Victoria University of Wellington

in fulfilment of the requirements for the degree of

Doctor of Philosophy

Victoria University of Wellington

2012 


\section{Abstract}

Pseudomonas aeruginosa, an increasingly multi-drug resistant human pathogen, is now one of the top three causes of opportunistic infection and there is much interest in identifying novel therapeutic targets for treatment. As a bacterial pathogen, $P$. aeruginosa encounters innate immune system defences and must continue to adapt to its defence strategies to accommodate the ever-changing environment. Though $P$. aeruginosa virulence determinants have been heavily characterised over the last several decades, most recent work acknowledges the complex interaction between the human host and the pathogen as an on-going dialogue of virulence factors adapting to the continuum that is the immune response. A major challenge that $P$. aeruginosa must overcome are reactive oxygen species (ROS) that are released at all stages of infection. Based on previous work which demonstrated a role for soluble nitro- and quinone oxidoreductase (NQOR) enzymes in protecting a related bacterium (Pseudomonas putida) from oxidative stress, we hypothesized that $P$. aeruginosa would similarly utilize NQORs to withstand ROS. This thesis seeks to understand the role of ROS-protecting enzymes in pathogenesis as well as their potential applications in a therapeutic context.

Several NQORs of $P$. aeruginosa were identified to possess biochemical characteristics consistent with the enzymatic capacity to indirectly reduce reactive species like $\mathrm{H}_{2} \mathrm{O}_{2}$. However, when individual genes encoding NQORs were deleted from $P$. aeruginosa, no apparent $\mathrm{H}_{2} \mathrm{O}_{2}$ sensitivity was seen. In contrast, when candidate genes were overexpressed, certain NQOR enzymes conferred the ability to tolerate $\mathrm{H}_{2} \mathrm{O}_{2}$ challenge at low concentrations; indicating that these NQORs may play a protective role whose effects are masked in vitro by genetic redundancy as well as a highly active endogenous catalase. By developing a novel in vivo cell culture infection model, the survival of $P$. aeruginosa post exposure to immunocompetent murine macrophages was also assessed. This not only demonstrated that several putative NQORs were activated in the presence of macrophages but also that an in vivo modelling system is likely to be more appropriate for discovering virulence determinants.

In a different aspect of this study it was investigated whether the reductive capacity of the $P$. aeruginosa-derived NQORs might hold potential for gene-directed enzymeprodrug therapy (GDEPT). Prodrugs, such as 5-(aziridin-1-yl)-2,4-dinitrobenzamide (CB1954) or the nitro-chloromethyl benzindoline SN 26438, are nontoxic in their native form, but become highly toxic upon reduction of their nitro functional groups. The $P$. aeruginosa NQORs, were tested to identify enzymes capable of efficient activation of CB1954 or SN 26438. Although none of these enzymes exhibited greater activity with CB1954 than the "best in class" Eschericha coli enzymes NfsA or NfsB, the $P$. aeruginosa $\mathrm{NfsB}$ orthologue (PA5190) demonstrated greater than 20-fold improved activity over $\mathrm{NfsB}$ from Escherichia coli in its ability to sensitise human cells to $\mathrm{SN}$ 26438. This finding offers promise for development of PA5190 and SN 26438 as a novel enzyme-prodrug paradigm for GDEPT. 


\section{Acknowledgements}

Many, many thanks are owed to C. Albright, G. Iaccarino, J. Vann, J. Squirrell, and P. Stoitzner for being the greatest mentors that ever were. Your encouragement and guidance are what kick-started my research career. Without this inspiration I may well have succumbed to the temptations of fame and fortune and would now, in all likelihood be living the tedious and no doubt troubled life of either an ex-Gold-Medal-winning Olympic speed-skater or possibly a Women's NBA superstar.

To my supervisors, Anne and Dave, with your patience, understanding, compassion and more than a healthy dose of prodding, I was able to make it to the finish line. Together you have allowed me the freedom to shape my own project and pursue an endeavour that at times appeared beyond the scope of possibility. I would never have made it to this point without Anne's pick-me-up "rays of sunshine" or Dave's unwavering confidence in my abilities. Thanks especially to Dave for pushing me so far beyond what I thought I was capable of in the last few weeks of write-up; I will assuredly appreciate this cruel form of torture in the weeks and months ahead once my sleep patterns have returned to normal and the caffeine levels in my blood have diminished.

I would like to thank the Health Research Council of New Zealand for my first several years of funding. I would also like to acknowledge the generous tax payers of this beautiful nation I now call home for footing the bill for my last two years of study. I promise to work hard to repay my substantial debt for the next many a year.

To my family, in New Zealand and America, you are all part of the team that made this thesis possible. To Beth and Robert, for welcoming me into your family and providing a home away from home to have "mini holiday weekends" and for the regular supply of cakes, casseroles and a perpetually full pantry to see me (mostly Paul) through the long days and late nights. To Mom and Dad for everything and all that you do, for being my sounding board, for wrapping me up and making things right. Without your unconditional love and steadfast belief in me, none of this would have been possible. To Mom for your amazingly themed care packages, for Twizzlers, cupcake mixes, and an endless supply of warm fuzzy socks! To Dad, for your FiveYear Plans and self-help books planted in my bedroom and for your wisdom and calm in the face of my frequent angst and agitation. To my brother Dave for my Beats Audio life-line and gangster rap that has helped get me through these last few months and for making Mom teach us chemistry; if we never had science class at home I may have grown up to be an accountant!

Thanks to Marijke for stress baking, to Vicky for helping to keep me sane, to Deanna for being my little slice of USA and allowing me American moments and to Jess for hilarious stories, dawn breakfasts in lycra and for showing how tennis can be an extreme sport.

And last, but by far not least, II mio Campionissimo e il mio amore, Paul, questa tesi è tua tanto quanto è mio. Thank you for spending late nights in the lab with me and car deliveries magically full of food. For getting me out of bed and out the door in the morning and letting me wear pyjamas in public. Thank you for glasses of wine, pots of coffee and for leisurely reading about enzymes then explaining the chemistry using jazz hands. My Champion of champions, my love, this thesis is as much yours as it is mine. 


\section{Table of Contents}

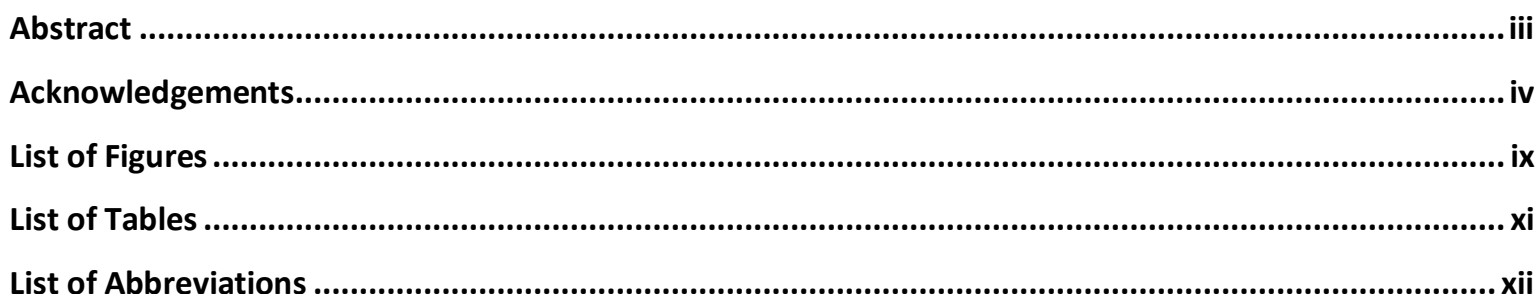

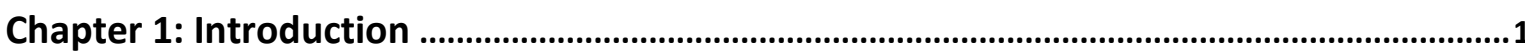

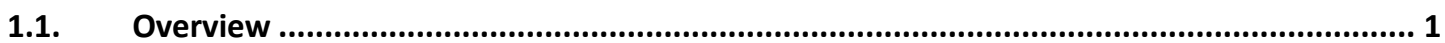

1.2. Pseudomonas aeruginosa, an opportunistic pathogen................................................... 1

1.2.1. Oxidative stress increases the prevalence of $P$. aeruginos $a$ infections ..................... 2

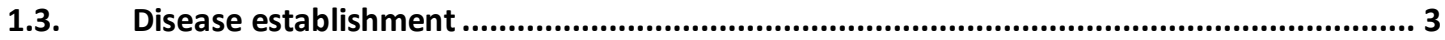

1.3.1. Airway epithelial cell barriers........................................................................... 3

1.3.2. P. aeruginosa evasion of epithelial defence mechanisms ...................................... 5

1.4. Phagocytosis and a dual role for ROS production .......................................................... 7

1.4.1. Oxygen-dependent killing mechanism of phagocytes....................................... 7

1.4.2. Mechanisms by which $P$. aeruginosa can withstand phagocytosis........................... 9

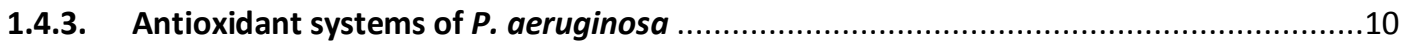

1.4.3.1. NAD(P)H-dependent nitro- and quinone oxidoreductases (NQOR) - alternative

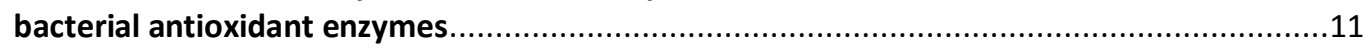

1.4.3.2. NQOR metabolism of nitro-aromatic compounds ....................................13

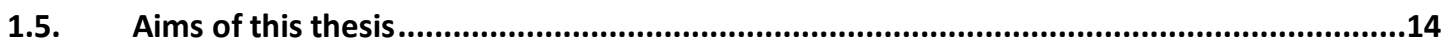

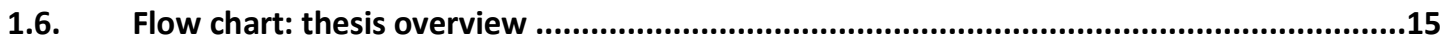

Chapter 2: General Methods and Materials .................................................................16

2.1. Chemicals, enzymes, reagents, media .........................................................................16

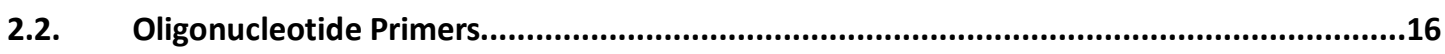

2.3. Bacterial strains (including clinical isolates) and plasmids.............................................18

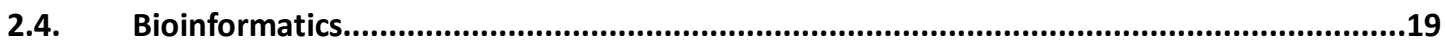

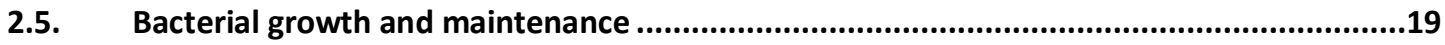

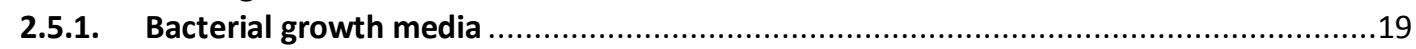

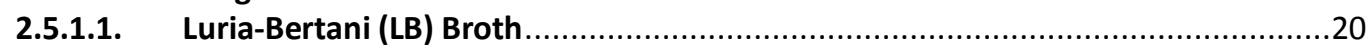

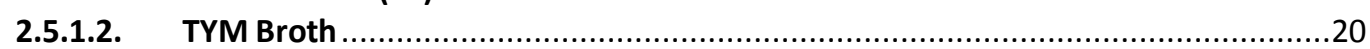

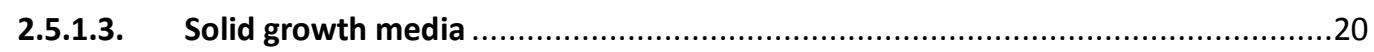

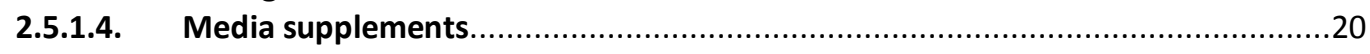

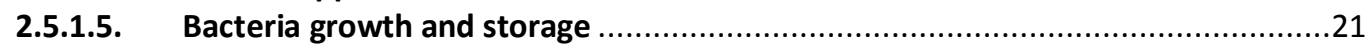

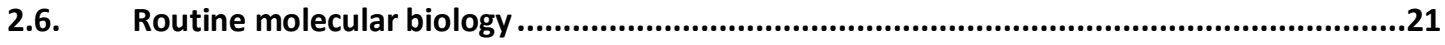

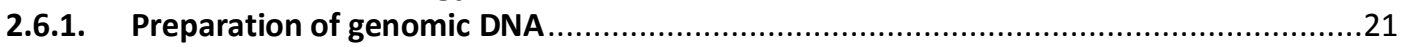

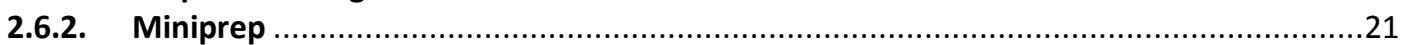

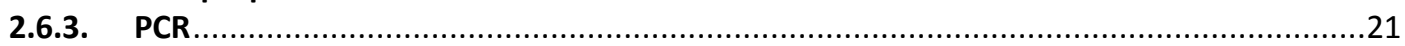

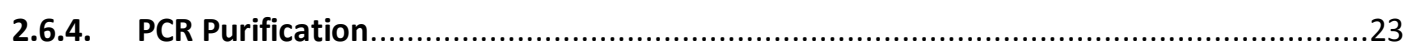

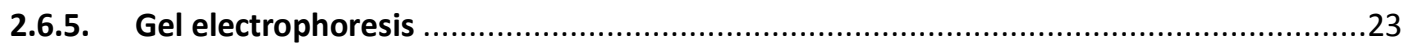

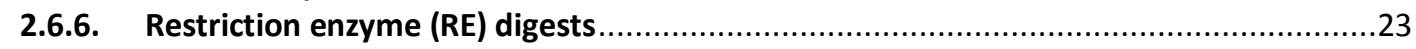

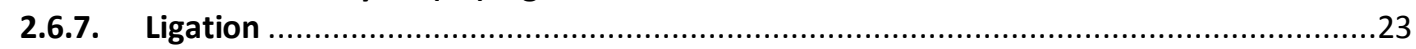

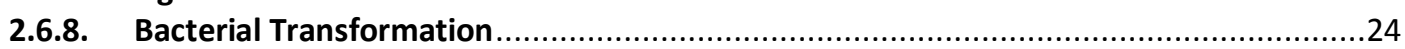

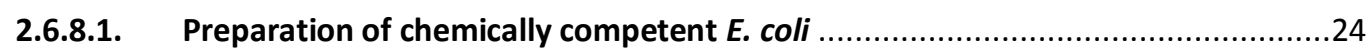




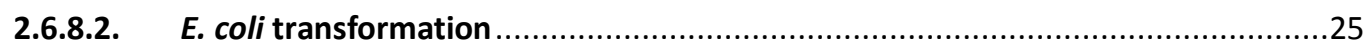

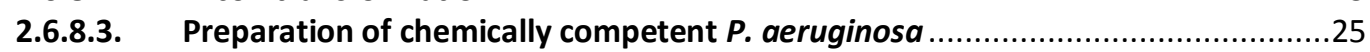

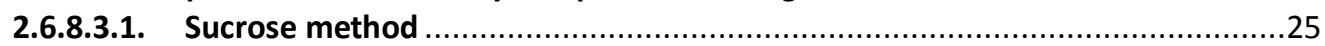

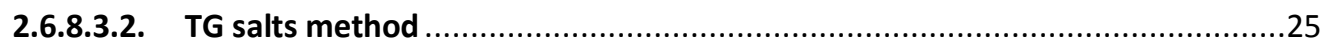

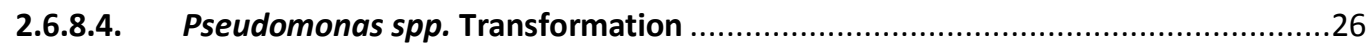

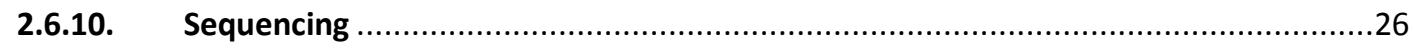

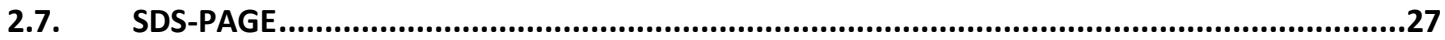

2.8. Purification of recombinant His6-tagged enzymes .......................................................28

2.8.1. E. coli enzyme expression and biochemical characterization ...................................28

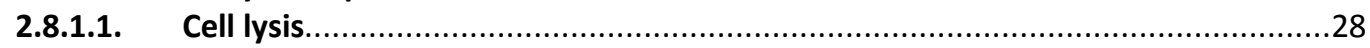

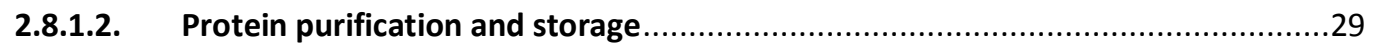

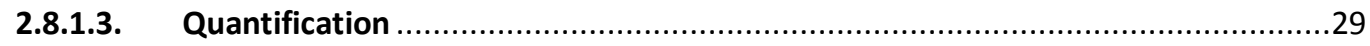

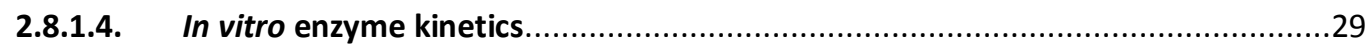

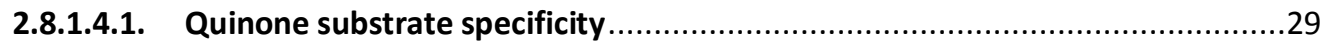

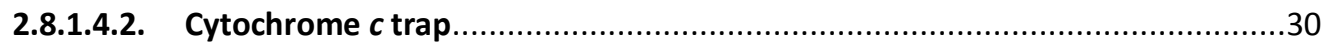

2.8.1.4.3. Determination of SN 26438 extinction coefficients ...................................30

2.8.1.4.4. In vitro purified NQORs kinetics with prodrug .....................................30

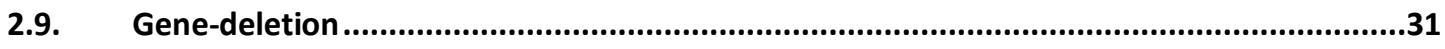

2.10. $\mathrm{H}_{2} \mathrm{O}_{2}$ challenge and Amplex ${ }^{\circledR}$ Red hydrogen peroxide assay.........................................34

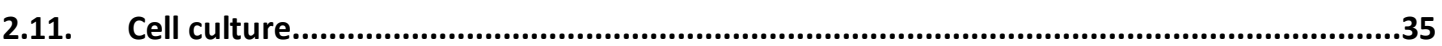

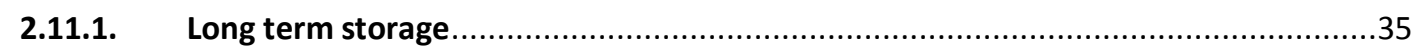

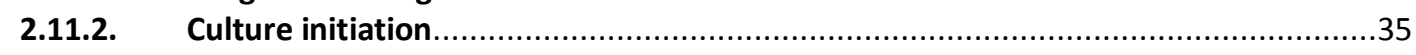

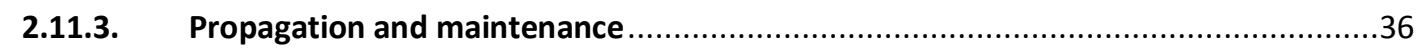

2.12. Reactive oxygen release by RAW-264.7 macrophages ...................................................36

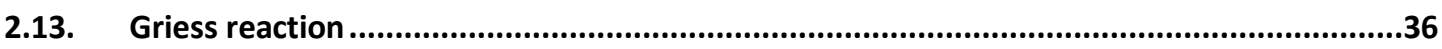

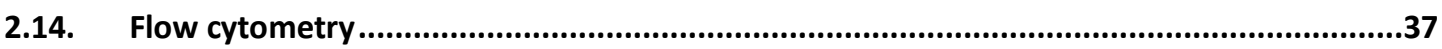

2.14.1. Live $P$. aeruginosa population analysis by flow cytometry - a gating strategy for

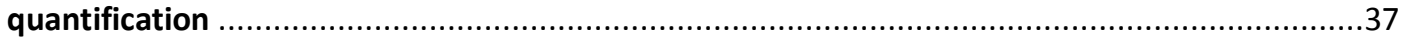

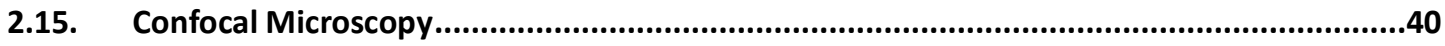

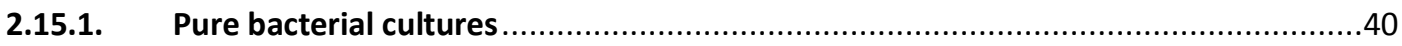

2.15.2. Mammalian cell monolayers ........................................................................ 40

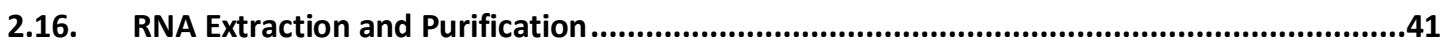

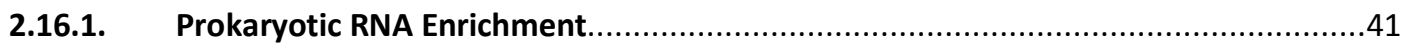

2.16.2. RNA Quality Control..................................................................................

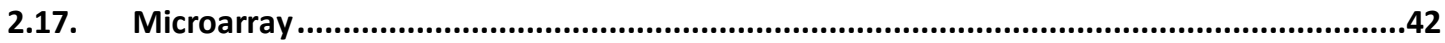

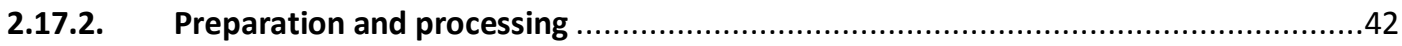

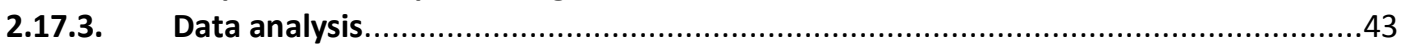

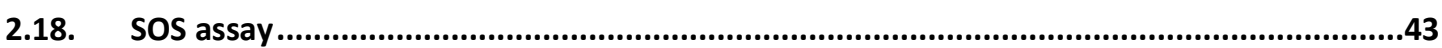

2.19. Sensitisation of HCT-116 cells to CB1954 and SN 26438 using E. coli expressing candidate NQORs

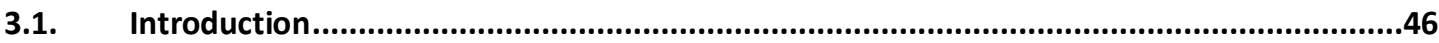

3.1.1. Soluble nitro- and quinone oxidoreductases .................................................

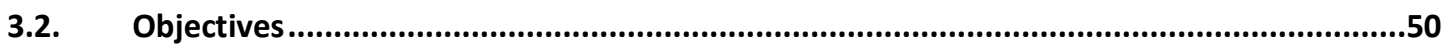

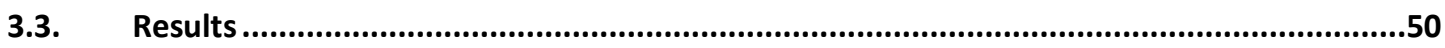

3.3.1. Biochemical characterisation of $P$. aeruginosa $\mathrm{NAD}(\mathrm{P}) \mathrm{H}$ quinone oxidoreductase

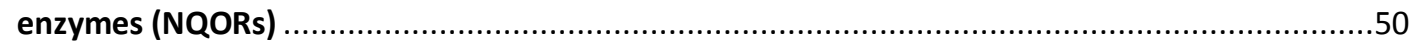

3.3.1.1. Selection of NQOR candidates for this study ........................................50 
3.3.1.2. In vitro quinone reduction by $P$. aeruginosa NQOR-like enzymes.......................51

3.3.1.3. Simultaneous two electron transfer by $P$. aeruginosa NQORs............................54

3.3.2. Assessment of $\mathrm{NQOR} \mathrm{H}_{2} \mathrm{O}_{2}$-protective phenotypes in vitro.....................................56

3.3.2.1. NQOR Gene-deletion...............................................................................59

3.3.2.2. Effect of $P$. aeruginosa NQOR over-expression on $P$. aeruginosa $P A O 1$ and $P$. putida $\mathrm{KT2440}$ survival in $\mathrm{H}_{2} \mathrm{O}_{2}$-amended media ...................................................60

3.3.2.3. Over-expression of PA2580 in P. aeruginosa PAO1 .....................................65

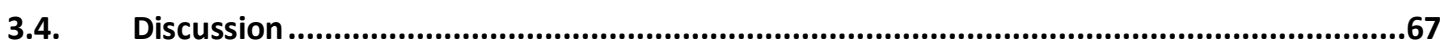

Chapter 4: The $\boldsymbol{P}$. aeruginosa response to challenge by murine macrophages

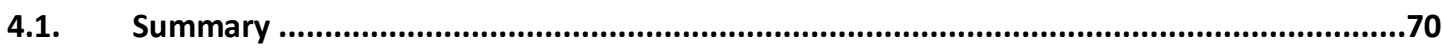

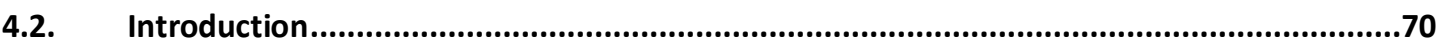

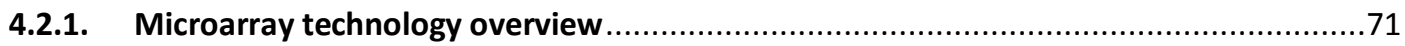

4.2.2. Previous microarray studies of oxidative stress in $P$. aeruginosa............................72

4.2.2.1. In vitro studies with oxidative stress as a constituent of culture media...............73

4.2.2.2. In vivo cell culture studies involving eukaryotic cell interaction with $P$. aeruginosa 74

4.3. Objectives .76

4.4. Development of an in vivo cell culture infection model to evaluate the $P$. aeruginosa response to challenge by murine macrophages

4.4.1. Using flow cytometry to quantify bacteria ........................................................77

4.4.1.1. Proof of Principle: Using flow cytometry and confocal microscopy to quantify antimicrobial properties of nanoparticle-coated wool .................................................. 80

4.4.2. Collection method of macrophage-engulfed $P$. aeruginosa .....................................82

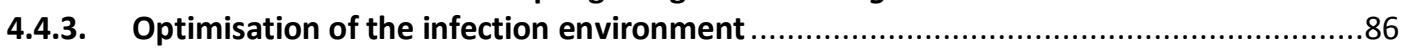

4.5. In vivo cell culture infection screening of $P$. aeruginosa strains ....................................92

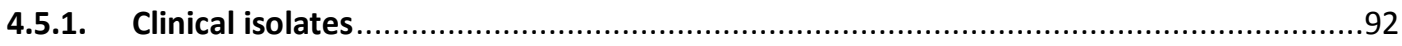

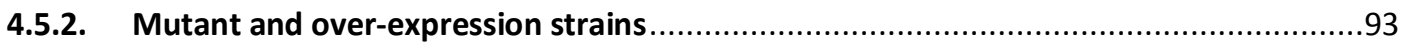

4.6. Modification of infection conditions for RNA isolation .................................................97

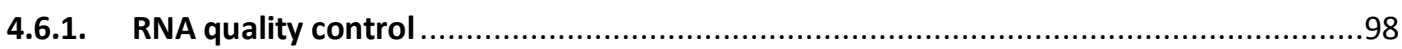

4.7. Transcriptional profile of internalised $P$. aeruginosa...................................................99

4.7.1. Interpretation and analysis of functional gene groups .....................................102

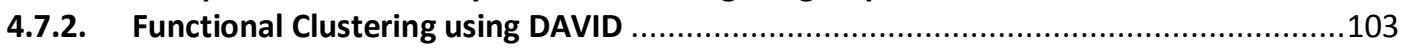

4.7.3. Expression data cross referenced with annotated operons in DOOR ......................105

4.7.3.1. The three P's: Pyocin, Pyoverdine, and Pyochelin .........................................107

4.7.3.2. Oxidoreductases activated by phagocytosis .................................................. 109

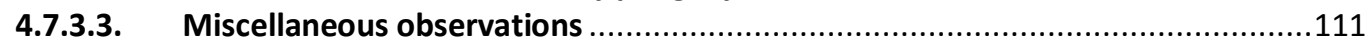

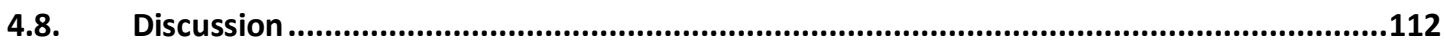

\section{Chapter 5: Activation of the nitro-chloromethyl benzindoline prodrug SN 26438 by}

P. aeruginosa NfsB-like NQOR: A novel enzyme-prodrug pairing for GDEPT.

5.1. Introduction.

5.2. Objectives.

5.3. Results ...............................................................................................................122

5.3.1. Evaluation of CB1954 and SN 26438-reducing activity of candidate $P$. aeruginosa NQORs through their ability to induce the $E$. coli SOS response......................................122

5.3.2. In vitro kinetics of purified NQOR candidates with CB1954 and SN 26438 .................125

5.3.3. Sensitisation of the human colon carcinoma cell line HCT-116 to CB1954 and SN 26438 using the in vivo cell culture $\mathrm{IC}_{50}$ assay 
5.3.4. Determination of the $P$. aeruginosa candidate PA5190 bystander effect using three

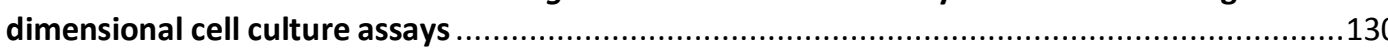

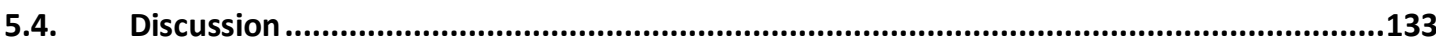

Chapter 6: Summary, conclusions, and future directions .....................................................136

6.1. Research Motivation and Summary ............................................................................136

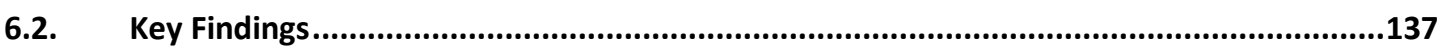

6.2.1. Potential functional redundancy of NQORS in $P$. aeruginosa..................................137

6.2.2. Development of an in vivo cell culture infection model for the isolation of RNA from viable, macrophage-engulfed $P$. aeruginosa ..........................................................138

6.2.3. The transcriptional response of macrophage-engulfed $P$. aeruginosa .....................138

6.2.4. PA5190 and SN 26438: A novel enzyme-prodrug pairing for GDEPT ......................139

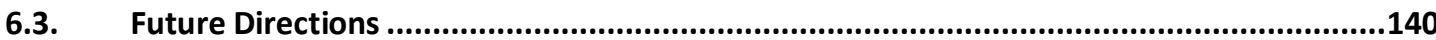

6.3.1. Elucidating a biological role for $P$. aeruginosa NQORs ............................................ 140

6.3.2. Implications of research for $P$. aeruginosa therapy ............................................. 140

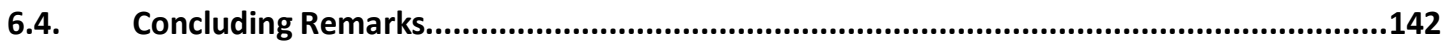

Appendix 1: Antimicrobial properties nanoparticle-coated wool fibres ...........................................143

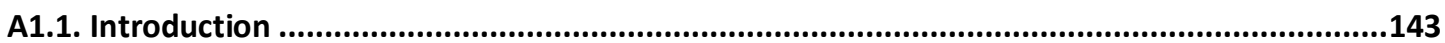

A1.2. Summary of findings............................................................................................................144

Appendix 2: Microarray Top Tables of Gene Expression.................................................................148

Appendix 3: Microarray Data Sets by Functional Classification ........................................................160

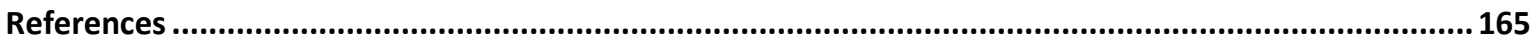




\section{List of Figures}

List of Figures

Figure 1.1. Summary of immunomodulation compounds released upon epithelia- $P$. aeruginosa contact 5

Figure 1.2. Schematic overview of quinone reduction .12

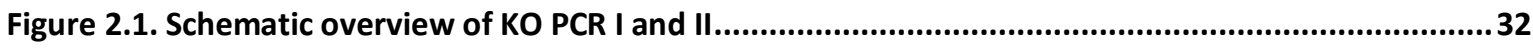

Figure 2.2. Schematic overview of primary and secondary recombinant integration ..................................34

Figure 2.3. P. aeruginosa flow cytometry controls ................................................................................. 38

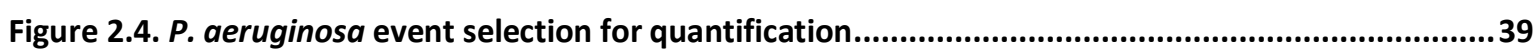

Figure 3.1. The major ROS components produced by the phagocyte-derived oxidative burst .....................46

Figure 3.2. $\mathrm{H}_{2} \mathrm{O}_{2}$-scavenging compared to growth in P. putida .....................................................................49

Figure 3.3. Purified His6 tagged NQORs on SDS-PAGE .........................................................................52

Figure 3.4. Structures of quinone substrates used to measure steady-state kinetic parameters .................53

Figure 3.5. Determination of simultaneous two electron transfer - cytochrome $c$ trap ...............................55

Figure 3.6. Growth of wild type $P$. aeruginosa PAO1 and P. putida $\mathrm{KT} 2440$ in $\mathrm{H}_{2} \mathrm{O}_{2}$ amended media ........57

Figure 3.7. Effect of $k a t A$ over-expression in different $P$. aeruginosa PAO1 and P. putida KT2440

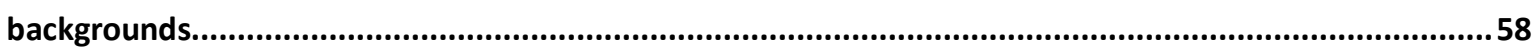

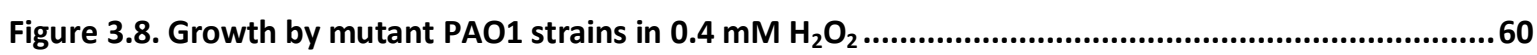

Figure 3.9. PA4975 and PA0949 over-expression in PAO1 .....................................................................62

Figure 3.10. The influence of the $\mathrm{pSX}$ over-expression vector on $\mathrm{H}_{2} \mathrm{O}_{2}$ tolerance by the $\Delta$ katA strain .......63

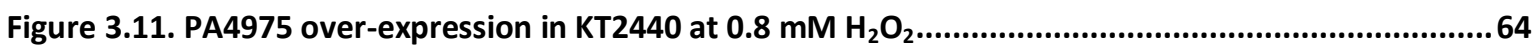

Figure 3.12. Conversion of $\mathrm{OD}_{660}$ to percentage growth for $\mathrm{PA2580}$ over-expressing $P$. aeruginosa PAO1 relative to the empty-plasmid control .66

Figure 4.1. Schematic overview of Affymetrix GeneChip ${ }^{\circledR}$ technology .....................................................71

Figure 4.2. P. aeruginosa quantification by flow cytometry and CFU .........................................................78

Figure 4.3. Live and dead PAO1 quantification by flow cytometry versus CFU counts ...............................79

Figure 4.4. Bacterial survival assessment by flow cytometry, CFU and confocal microscopy .....................81

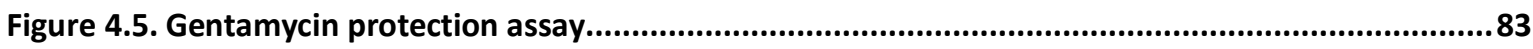

Figure 4.7. Total prokaryotic RNA recovered from RAW-264.7 macrophage-engulfed $P$. aeruginosa .........85

Figure 4.8. Confocal images of RAW-264.7 infected with P. aeruginosa ....................................................... 86

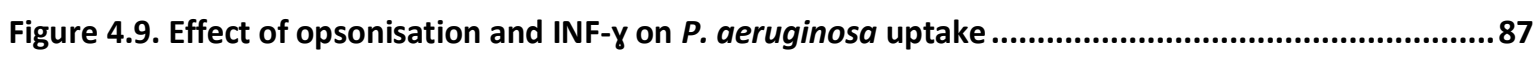

Figure 4.10. Recovery of viable $P$. aeruginosa post-phagosomal maturation ..........................................8

Figure 4.11. Viability of PAO1 during phagosomal maturation at MOIs of 50 and 100 ..............................90

Figure 4.12. Phenotypic changes of PAO1 viabilty (MOI 50) as represented on cytogram ..........................91

Figure 4.13. Viable $P$. aeruginos $a$ clinical strains recovered from macrophage infection............................93

Figure 4.14. Viable $P$. aeruginosa NQOR-deletion and NQOR over-expression strains recovered from macrophage infection 
Figure 4.17. Distribution of activated and repressed genes between Internal PAO1 and External

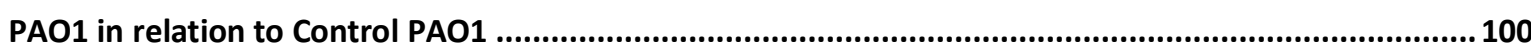

Figure 4.18. Functional classification of Internal PAO1 genes ...........................................................101

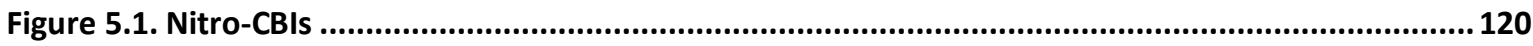

Figure 5.2. Schematic representation of the bystander effect ............................................................121

Figure 5.3. Expression of $P$. aeruginosa NQORs in SOS-R2 ..................................................................123

Figure 5.4. CB1954- and SN 26438-induced SOS responses from NQOR over-expression strains of SOS-R2

Figure 5.5. Expression of E. coli NTRs in the human colon carcinoma cell line HCT-116 as detected by

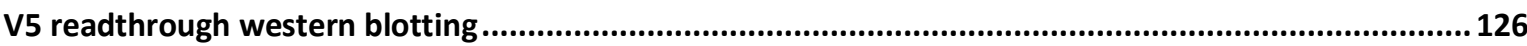

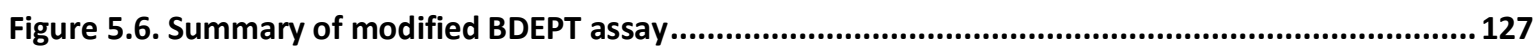

Figure 5.7. HCT-116 human carcinoma BDEPT model assay................................................................129

Figure 5.8. Enhanced bystander efficiency of SN 26438 was demonstrated with PA5190-dependent activation compared to $\mathrm{NfsB}$ 


\section{List of Tables}

Table 2.1.Oligonucleotide primers used in this study ...............................................................................16

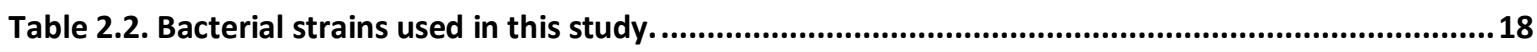

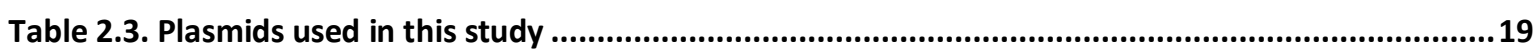

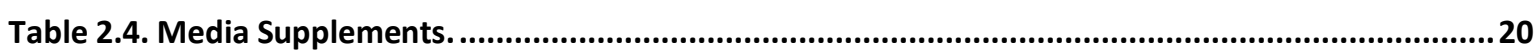

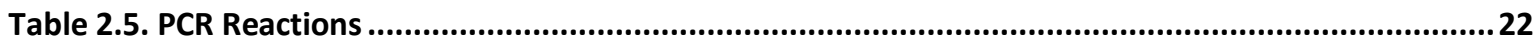

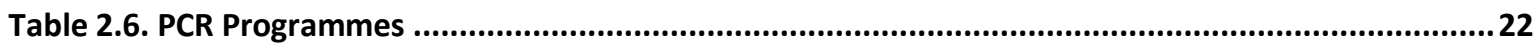

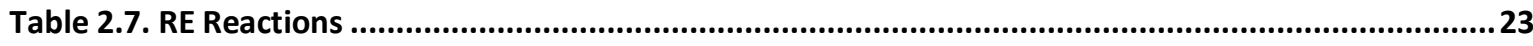

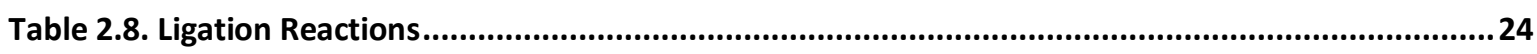

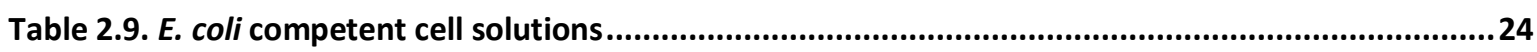

Table 2.10. P. aeruginosa competent cell solutions (TG salts) ......................................................................26

Table 2.11. SDS-PAGE materials ................................................................................................................27

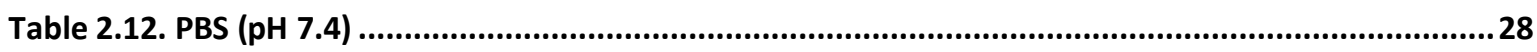

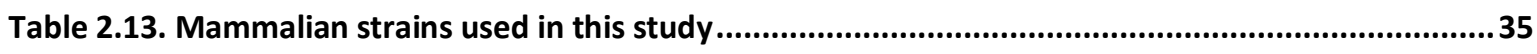

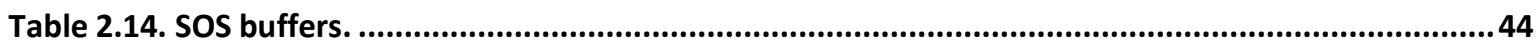

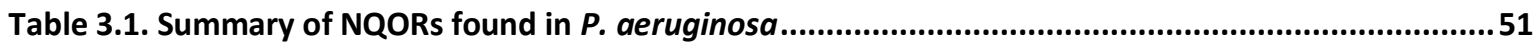

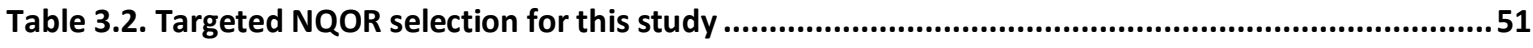

Table 3.3. Steady-state Michaelis-Menten kinetic parameters..............................................................53

Table 4.1. Summary of PAO1 microarrays investigating oxidative stress in vitro.......................................73

Table 4.2. Summary of PAO1 microarrays involving eukaryotic cell interaction...........................................75

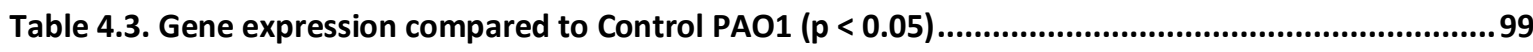

Table 4.4. Internal PAO1 up-regulated functional clustering ...................................................................... 104

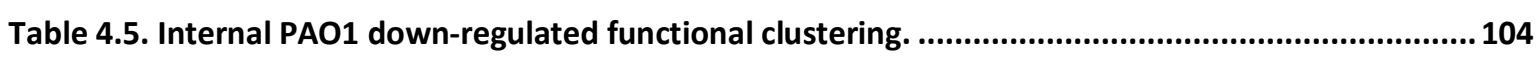

Table 4.6. Transcriptional regulators (Internal PAO1 up-regulated data set) ...............................................106

Table 4.7. Putative NQORs identified in Internal PAO1 up-regulated operons ..........................................110

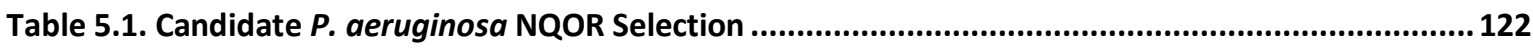

Table 5.2. Steady-state kinetics of purified enzymes for CB1954 reduction...........................................125

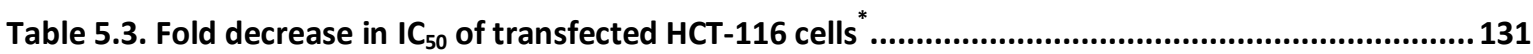




\section{List of Abbreviations}

\begin{tabular}{|c|c|}
\hline ACSRC & Auckland Cancer Society Research Centre \\
\hline Amp & Ampicillin \\
\hline BDEPT & Bacteria directed enzyme prodrug therapy \\
\hline BEE & Bystander effect efficiency \\
\hline BLAST & Basic Local Alignment Search Tool \\
\hline BQ & Benzoquinone \\
\hline BSA & Bovine Serum Albumin \\
\hline CB1954 & (5-(aziridin-1-yl)-2,4-dinitrobenzamide) \\
\hline Chl & Chloramphenicol \\
\hline CMEM & Dubelco's Modified Eagle Media amended with FCS and HEPES \\
\hline CMEM-AIG & CMEM amended with ampicillin, IPTG, and glucose \\
\hline CM-H2DCFDA & $\begin{array}{l}\text { 5-(and-6)-chloromethyl-2',7'-dichlorodihydrofluorescein diacetate, } \\
\text { acetyl ester }\end{array}$ \\
\hline CoQ1 & Coenzyme Q1 \\
\hline DAVID & Database for Annotation, Visualization and Integrated Discovery \\
\hline DMSO & Dimethylsulfoxide \\
\hline DOOR & Database of prOkaryotic OpeRons \\
\hline e- & Electron \\
\hline ESR & Environmental Science \& Research \\
\hline FACS & Fluorescence-activated cell sorting \\
\hline FAD & Flavin adenine dinucleotide \\
\hline FCS & Foetal calf serum \\
\hline FMN & Flavin mononucleotide \\
\hline FSC & Forward side scatter \\
\hline GDEPT & Gene-directed enzyme-prodrug therapy \\
\hline GFP & Green fluorescent protein \\
\hline Gm & Gentamycin \\
\hline $\mathrm{H}_{2} \mathrm{O}_{2}$ & Hydrogen peroxide \\
\hline HEPES & (4-(2-hydroxyethyl)-1-piperazineethanesulfonic acid) \\
\hline $\mathrm{IC}_{50}$ & $\begin{array}{l}\text { Concentration of test compound required to inhibit growth of test } \\
\text { organism to } 50 \% \text { of control (unchallenged) levels }\end{array}$ \\
\hline IL & Interleukin \\
\hline IPTG & Isopropyl-D-thiogalactoside \\
\hline Kan & Kanamycin \\
\hline$k_{\text {cat }}$ & $\begin{array}{l}\text { The turnover number fo an enzyme giving the number of substrate } \\
\text { molecules converted to product per enzyme active site per unit of time. }\end{array}$ \\
\hline$\kappa_{m}$ & $\begin{array}{l}\text { Kinetic constant describing the concentration of substrate at which rate } \\
\text { of catalysis of a specific enzyme is exactly half of the } V_{\text {max }} \text {, under the } \\
\text { conditions tested. }\end{array}$ \\
\hline KO & Knockout \\
\hline LB & Luria-Bertani \\
\hline LB-AIG & Luria-Bertani broth with ampicillin, IPTG, and glucose \\
\hline LpDH & Lipoyl dehydrogenase \\
\hline
\end{tabular}




$\begin{array}{ll}\text { LPS } & \text { Lipopolysaccharide } \\ \text { MCL } & \text { Multi-cell layer } \\ \text { Men } & \text { Menadione } \\ \text { MIMR } & \text { Malaghan Institute for Medical Research } \\ \text { MOI } & \text { Multiplicity of infection } \\ \text { NADH } & \text { Nicotinamide adenine dinucleotide } \\ \text { NADPH } & \text { Nicotinamide adenine dinucleotide phosphate } \\ \text { NCBI } & \text { National Centre for Biotechnology Information } \\ \text { Nitro-CBI } & \text { Nitro-chloromethylbenzindolines } \\ \text { NO } & \text { Nitric oxide } \\ \text { NQOR } & \text { Nitro- and quinone oxidoreductase } \\ \text { O2 } & \text { Superoxide } \\ \text { OD } & \text { Optical density (at a certain wavelength) } \\ \text { OH } & \text { Hydroxyl radical } \\ \text { ORF } & \text { Open reading frame } \\ \text { PCR } & \text { Polymerase chain reaction } \\ \text { PI } & \text { Propidium iodide } \\ \text { PMN } & \text { Polymorphonuclear leukocyte } \\ \text { RIN } & \text { RNA integrity number } \\ \text { ROS } & \text { Reactive oxygen species } \\ \text { rpm } & \text { Revolutions per minute } \\ \text { SN 26438 } & \text { Nitro-CBl analogue } \\ \text { SOD } & \text { Superoxide dismutase } \\ \text { SSC } & \text { Side scatter } \\ \text { TLR } & \text { Toll like receptor } \\ \text { Tris } & \text { Tris(hydroxymethyl)aminomethane } \\ \text { V/v } & \text { Volume per volume } \\ \text { W/v } & \text { Weight per volume } \\ & \end{array}$




\section{Chapter 1: Introduction}

\subsection{Overview}

The research described in this thesis began with a biochemical and genetic investigation into a novel antioxidant defence mechanism, aiming to discern whether the opportunistic bacterial pathogen Pseudomonas aeruginosa might rely on a substantial contribution from soluble quinone oxidoreductase enzymes to withstand assault by host-generated oxidative stress during the course of pathogenesis. This preliminary investigation (described in Chapter 3) provided a foundation for two quite different follow up studies, one examining more globally the response of $P$. aeruginosa to challenge by host macrophages (Chapter 4), and the other looking at potential applications of the oxidoreductase enzymes in activation of prodrugs for cancer gene therapy (Chapter 5). Owing to the quite different focus of each of these arms of the study, detailed chapter-specific introductions have been written for each of them. In contrast, this Introduction chapter seeks to provide a more general overview of how $P$. aeruginosa causes infection and the importance of reactive oxygen species to that process.

\subsection{Pseudomonas aeruginosa, an opportunistic pathogen}

Pseudomonas aeruginosa, a Gram-negative bacterium, has emerged as major cause of life-threatening secondary infections in medical care facilities. It is an opportunistic pathogen accounting for over $10 \%$ of all hospital-acquired infections (1). Its genetic flexibility allows $P$. aeruginosa to survive in almost all environments and with minimal nutrition, resulting in an organism that is (i) phenotypically unstable and difficult to track epidemiologically, (ii) capable of persisting in different physical settings from community to hospital settings, and (iii) efficient in developing multi-drug resistance (2). P. aeruginosa is now one of the top three causes of opportunistic human infection and there is much interest in identifying novel therapeutic targets for treatment (3).

In the US alone, care and treatment associated with hospital-acquired infections costs the medical community $\$ 28-34$ billion per annum (4). With hospital-acquired 
infections now the $4^{\text {th }}$ leading cause of death in the US (5), $P$. aeruginosa has become a key pathogen considered in medical infection control plans and is particularly prevalent in immunocompromised patients suffering from AIDS, cystic fibrosis, cancer, pneumonia, or those recovering from surgery (3). P. aeruginosa is also the most common Gram-negative bacterium isolated from nosocomial infections. The International Federation for Infection Control found that "at any one time about 1 in 10 patients in acute care hospitals have a nosocomial infection, and an additional 10$60 \%$ of infections may present after discharge" (6).

\subsubsection{Oxidative stress increases the prevalence of $P$. aeruginosa infections}

Mechanical ventilation is a necessity for patients with respiratory difficulties. However, hyperoxia ( $95 \% \mathrm{O}_{2}$ exposure) brought on by mechanical ventilation, vastly increases the occurrence of $P$. aeruginosa infections $(2,7-9)$. $P$. aeruginosa has been identified as the causative agent for most ventilator associated pneumonias that commence post mechanical ventilation $(10,11)$. Additionally, persons living with cystic fibrosis (CF) commonly require ventilation assistance and chronic $P$. aeruginosa infections are the primary cause of morbidity and mortality for CF patients $(12,13)$. Due not only to the primary disease of the patient but also because of mechanical ventilation, $P$. aeruginosa has become the principal species colonizing CF tracheal aspirates (14).

The incidence of pseudomonal pneumonia brought on by ventilation further increases in patients who had previously had some type of antibiotic course $(8,9)$, as a consequence of this organism's propensity for multi-drug resistance. $P$. aeruginosa is naturally resistant to most clinically available antibiotics making it difficult to treat; and surveillance finds the prevalence of the organism in nosocomial infections increasing despite aggressive antibiotic administration $(7,12,15)$.

In addition to multi-drug resistance, $P$. aeruginosa produces a diverse array of virulence factors that may contribute to survival in the presence of hyperoxia and/or antimicrobial treatment; however, although such virulence determinants have been heavily characterised over the last several decades, most recent work acknowledges the complex interaction between the human host and the pathogen as an on-going dialogue of virulence factors adapting to the continuum that is the immune response. When surveying the literature from both a microbe-focused and immunological 
perspective, it appears increasingly likely that a key offensive mechanism of $P$. aeruginosa may be to antagonise the human inflammatory immune response as one stage in establishing disease. This introduction aims to present an overview of selected host- $P$. aeruginosa interactions that may underpin a role for oxidoreductase enzymes in oxidative stress protection.

\subsection{Disease establishment}

$P$. aeruginosa requires a breakdown of the skin or mucosal barriers or an impaired immune system to establish disease (16). Like most pathogenic bacteria, $P$. aeruginosa is assumed to have evolved a progression of virulence factors to adapt to and overcome the ever changing host immune responses. Many of these virulence factors have been studied in isolation by reductionist approaches as well as in more complex in vivo investigations. Still, there are "significant gaps in the understanding of how $P$. aeruginosa survives the inflammatory-rich environment" (17). It has been hypothesised that $P$. aeruginosa infections comprise three stages: (i) attachment and colonisation, (ii) local invasion, and (iii) systemic disease. Each of these three stages are regulated by bacterial virulence factors and these virulence factors are involved in the development of the specific syndromes (i.e. different forms of the disease; (18)).

\subsubsection{Airway epithelial cell barriers}

The role of the host epithelial cell barrier has been studied primarily in the context of P. aeruginosa colonisation of cystic fibrosis patients. Upon initial interaction with the bacteria, the host epithelial cells mount a range of antimicrobial defence mechanisms to prevent $P$. aeruginosa attachment and colonisation (19). The first line of defence is the physical barrier of tight junctions between the epithelial cells, which, are impermeable in normal tissue $(20,21)$. Secondly, epithelial cells can release antimicrobials like lactoferrin and ß-defensin, both cationic proteins capable of bacteriostatic and/or bactericidal activities (22-24). Epithelial cells also have a demonstrated ability to release reactive oxygen species (ROS; (25-27)). Like antimicrobial peptides, ROS have a dual function in that they can peform bactericidal activities as well as stimulate the release of cytokines and chemokines to attract 
immune active cells to the site of infection $(28,29)$. Additionally, epithelial cells release other immunomodulatory compounds like interleukin (IL)-8, IL-10, tumour necrosis factor alpha (TNF- $\alpha)$, and interferon gamma (IFN- $\gamma$ ) which help regulate antigen presentation, leukocyte recruitment and activation (Figure 1.1).

In mammals, the first line of active defence against bacterial invasion is the polymorphonuclear leukocyte (PMN) population including neutrophils and macrophages. These specialised white blood cells quell imposing bacteria through phagocytosis, and by release of microbiocidal compounds (16). The first stage in this process occurs during the contact phase of infection where $P$. aeruginosa interacts with and binds receptors in epithelial cells and resident phagocytic cells, signalling the commencement of the complement cascade (30). Activation of the complement cascade releases a rigorous series of effector proteins that coat the bacterial membrane - collectively referred to as opsonins - allowing recognition of bacteria by phagocytes (31-33). The release of complement compounds impact the continued immune system in three ways: (i) by recruiting additional immune cells to the site of infection, (ii) by aiding in pathogen recognition (opsonisation), and (iii) by creating a multi-opsonin membrane attack complex which "punctures" the bacterial membrane leading to cell death.

Opsonins act primarily by masking the ionic charge of the bacterial plasma membrane. With both the bacterial and phagocytic membranes being negatively charged, it is otherwise difficult for the two cells to come together $(34,35)$. By concealing the bacterium's membrane charge, phagocytes are more adept at initiating phagocytosis (36-38). Additionally, once phagocytic-opsonin recognition has occurred, the activated immune cell can then stimulate proximally located phagocytes to upregulate opsonin-specific receptors (39). 


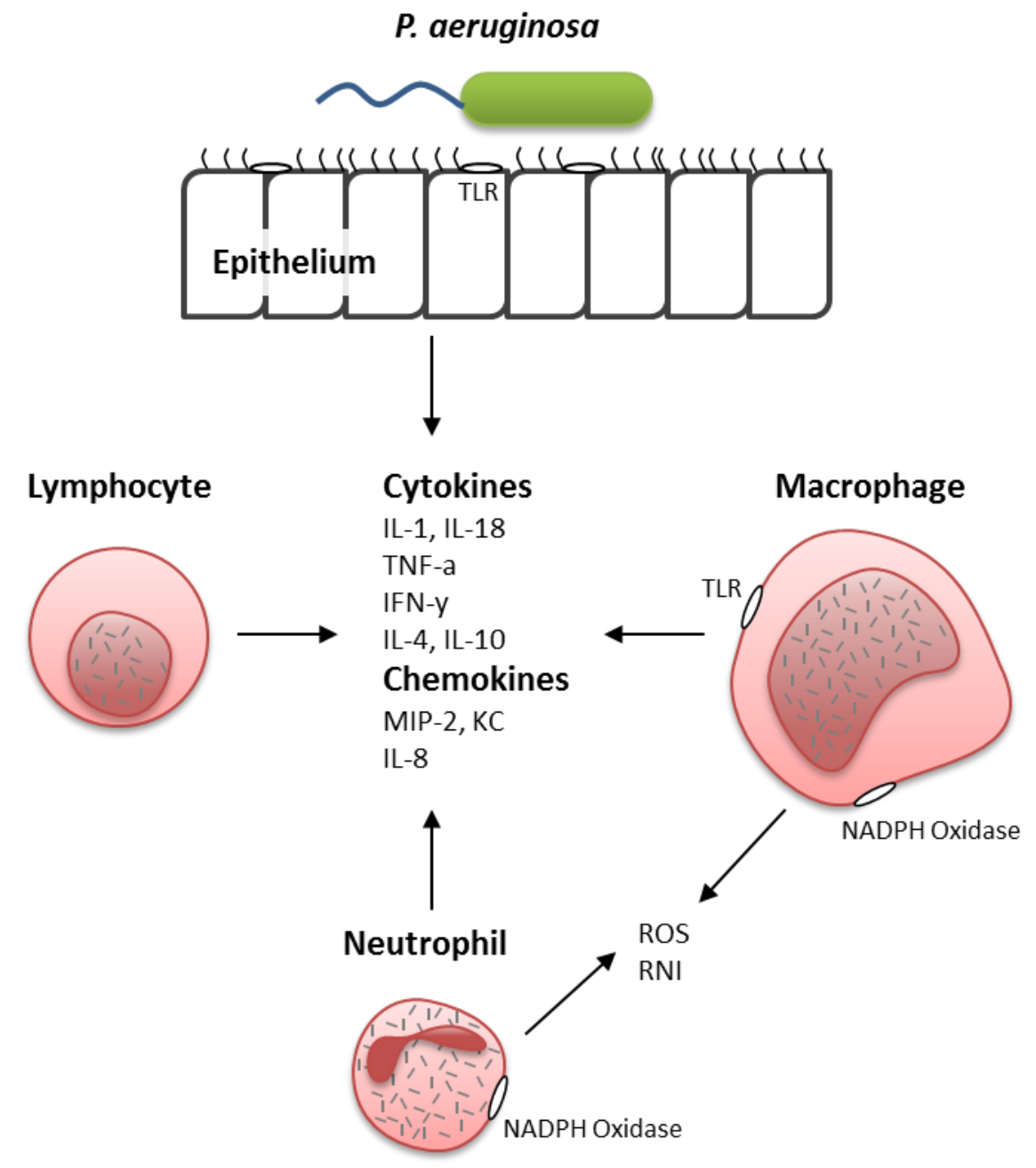

Figure 1.1. Summary of immunomodulation compounds released upon epithelia- $\boldsymbol{P}$. aeruginosa contact Image based on Sadikot et al (40).

\subsection{2. $P$. aeruginosa evasion of epithelial defence mechanisms}

In the context of a cystic fibrosis-like infection, non-mucoid $P$. aeruginosa are thought to be the first form of $P$. aeruginosa to colonise the injured airway after mucociliary clearance (40). To colonise the epithelia, and subsequently injure the tight junctions and disseminate systemically, $P$. aeruginosa enacts multiple defence strategies to withstand challenge by the host; and for each stage of infection the bacteria must continue to adapt its strategies to the ever changing immune environment.

Furthermore, the invading bacteria must continue to compete for growth-essential iron and avoid phagocytic leukocytes (40). It is here at the epithelial interface where $P$. aeruginosa cell-associated virulence factors like the flagellum and pili initiate the host 
system's innate immune response. Both $P$. aeruginosa pili and flagella act as ligands to bind epithelial toll like receptors (TLR) $(41,42)$. Different receptors have different virulence determinants, but both pili and flagellar interactions alike stimulate downstream immune inflammatory actions. For example, the pilus can bind the epithelial TLR-2 which not only stimulates proinflammatory expression but also enables attachment to the host, creating a pathway for injecting toxins into the epithelia, which causes damage and a means of entry past the tight junction barrier $(41,43,44)$. The flagella of $P$. aeruginosa can bind epithelial TLR-5 which stimulates IL-8 production (Figure 1.1; (2)). IL-8 is a chemoattractant of macrophages and induces ROS generation (45). Flagella and pili can also act as ligands for phagocytic cells (e.g. resident macrophages) which stimulate the recruitment of neutrophils to the site of infection (40).

P. aeruginosa also releases extracellular virulence factors to combat epithelial defence mechanisms. These consist of protolytic protease and elastase enzymes which act on the fibrin and elastin-rich adherence molecules between epithelia, thus disrupting the tight junctions and increasing permeability to bacteria $(40,46-48)$. Persistent production works to break down the epithelial tight junction barrier thereby establishing a port of entry for $P$. aeruginosa to enter the blood stream, ultimately resulting in sepsis.

Concomitant with attachment to TLRs, toxin injection, protease production, and the signalled recruitment of inflammatory response cells like macrophages and neutrophils, vascular leakage (oedema) occurs to allow passage of phagocytes from the blood to infected tissue. P. aeruginosa in turn exploits this oedema-induced swelling as yet another entry point to the blood stream causing sepsis $(49,50)$. This dialogue between $P$. aeruginosa, the epithelia and immunocompetent cells (macrophages, neutrophils, etc.) operates in a continual feedback loop prolonging the immune system-driven inflammation to cause extensive tissue damage.

Moreover, it has been shown that mucus production promotes and prolongs infection. Whereas non-mucoid $P$. aeruginosa are thought to be the first to directly interact with host epithelial cells by attachment, in many cases $P$. aeruginosa must undergo a conversion to the mucoid form, attributed by high alginate production $(51,52)$. In the conversion to this mucoid form, $P$. aeruginosa releases its flagella and the O-saccharide chain of the lipopolysaccharide, thus preventing TLR stimulation and 
therefore impeding recognition by phagocytes (40). The slimy surface from alginate production is also believed to make it harder for opsonins to "stick" to bacteria, further promoting immune system avoidance (53). Finally, the phenotypic conversion to alginate production has been shown to mediate $P$. aeruginosa antibiotic resistance by aiding in the development of biofilm (55-57) and to play a role in defending against $\operatorname{ROS}(40,58)$.

\subsection{Phagocytosis and a dual role for ROS production}

In the process of phagocytosis, the phagocyte changes morphology in response to encountering invading bacteria and engulfs the foreign organism by "wrapping" its plasma membrane around the bacterium. This phagocytic vesicle then combines with a lysosome to form the phagolysosome - a contained, plasma membrane enclosed environment where microbiocidal toxin release is confined to protect the rest of the phagocyte from experiencing self-damage.

Although the exact killing methods vary by cell type, they can be broadly classified as either oxygen-independent or oxygen-dependent systems. Macrophages can secrete over 100 different types of substances into their environment (59) and utilize both oxygen-dependent and independent mechanisms for bacterial control and killing. Oxygen-independent killing by macrophages appears to primarily involve the release of basic cationic proteins. It is known, however, that few of those molecules can directly cause damage to the bacterial membrane and are therefore causing death in an unknown manner $(60,61)$.

\subsubsection{Oxygen-dependent killing mechanism of phagocytes}

Upon binding bacteria, the macrophage dramatically increases its $\mathrm{O}_{2}$ consumption $10-$ 20 fold relative to the normal "resting" state $(62,63)$. This elevated oxygen uptake is not related to increases in mitochondrial respiration but instead the instigation of oxygen-dependent killing. The oxygen uptake depends on the activation of a plasma membrane bound enzyme complex which uses NADPH as an electron donor to convert $\mathrm{O}_{2}$ to superoxide $\left(\mathrm{O}_{2}{ }^{-}\right)$, released on the extracellular surface. Since the phagosome is essentially formed by "wrapping" the plasma membrane around engulfed bacteria, the 
phagocyte cytoplasm is protected from the high flux of $\mathrm{O}_{2}{ }^{-}$as this charged radical cannot passively diffuse across the lipid-rich membrane.

The $\mathrm{O}_{2}{ }^{-}$accumulation within the phagolysosome also results in a simultaneous influx of protons into the phagolysosome which are consumed to generate hydrogen peroxide $\left(\mathrm{H}_{2} \mathrm{O}_{2}\right)$. The initial $\mathrm{pH}$ rises from 7.4 to 7.8 during proton consumption. This is essential for the activation of superoxide dismutase, which converts $\mathrm{O}_{2}{ }^{-}$to the highly reactive $\mathrm{H}_{2} \mathrm{O}_{2}$. The $\mathrm{pH}$ of the phagolysosome then steadily decreases to 6.0-6.5 over the following $2 \mathrm{~h}$, as protons continue to enter the phagolysosome $(64,65)$. This collective release of concentrated ROS is referred to as the respiratory burst.

Following engulfment by macrophages $P$. aeruginosa is therefore exposed to considerable oxidative stress $(68,69)$, however, it is unlikely that increased superoxide concentration directly kills bacteria due to its inability to cross cellular membranes and its low reactivity in aqueous solution. $\mathrm{H}_{2} \mathrm{O}_{2}$, on the other hand, is a major constituent of the ROS generated within the phagolysosome and, being uncharged, is capable of crossing membranes making it more likely to be the source of direct bacterial killing (67). While $\mathrm{H}_{2} \mathrm{O}_{2}$ may not directly damage DNA, in the presence of certain metal ions, $\mathrm{H}_{2} \mathrm{O}_{2}$ is quickly converted via Fenton chemistry to the hydroxyl radical $\left(\mathrm{OH}^{\bullet}\right)$. The hydroxyl radical can directly attack DNA by interacting with both the purine and pyrimidine bases of DNA to form adducts $(68,69)$. $\mathrm{OH}^{\bullet}$ can either disrupt the deoxyribonucleic sugar ring or add to double bonds to the bases thereby creating $\mathrm{OH}$ adduct radicals and interfering with DNA replication and transcription $(68,70)$. $\mathrm{OH}^{\bullet}$ also fragments the deoxyribose sugar backbone and attacks proteins. When sugar-derived and protein-derived radicals meet, crosslinks can be formed thus interfering with protein folding, DNA repair, replication and transcription (71).

$\mathrm{H}_{2} \mathrm{O}_{2}$ is generated at low levels during normal cellular respiration, however, when responding to bacteria, $\mathrm{H}_{2} \mathrm{O}_{2}$ release can swell $\sim 80$ fold above metabolic levels in granuloctyes $(72)$ and $>1000$ fold by PMNs $(73)$ thus inhibiting bacterial growth $(74,75)$. After bacterial internalization by the phagocyte, the membrane-bound enzyme respiratory burst oxidase, catalyses consecutive one electron reductions of oxygen to produce $\mathrm{H}_{2} \mathrm{O}_{2}$ within the phagosome potentially exposing the bacteria to even higher concentrations of $\mathrm{H}_{2} \mathrm{O}_{2}$ than have been measured extracellularly (76). Although it is currently not possible to quantitate levels of $\mathrm{H}_{2} \mathrm{O}_{2}$ released within intracellular spaces (i.e. mitochondria and phagosomes), qualitative observations made using redox 
sensitive dyes have demonstrated rapid increase in $\mathrm{H}_{2} \mathrm{O}_{2}$ levels within organelles immediately after application of ROS generating compounds (77).

$\mathrm{H}_{2} \mathrm{O}_{2}$, as well as being a potent antimicrobial, is a natural trigger of the innate immune system (78). $\mathrm{H}_{2} \mathrm{O}_{2}$, at low sub millimolar concentrations, signals increases in vascular permeability, macrophage proliferation, and stimulates macrophages to adhere, as adherent cells are better at making ROS (79). It has been demonstrated that even bacteria that are actively expressing strong antioxidant systems do not completely eliminate ROS but maintain a low level balance between oxidant and antioxidant $(80,81)$. It has been hypothesised that cells like B-lymphocytes perhaps use the NADPH oxidase systems to communicate through $\mathrm{O}_{2}^{-}$production (70). ROS also stimulates IL-8 secretion by epithelia which feed back into macrophage recruitment, thereby completing a cycle of phagocytosis and ROS production (54).

\subsubsection{Mechanisms by which $P$. aeruginosa can withstand phagocytosis}

$P$. aeruginosa has, within its repertoire of virulence factors, a broad selection of antiphagocytic options to draw upon when exposed to immunocompetent and ROSproducing cells. These potentially include mechanisms to quench reactive species, to directly damage the attacking host cell, or to interfere with the phagocytic process at some stage (e.g. recognition and uptake). For example, as noted above, the production of alginate in biofilm formation masks the surface receptors (opsonins) required for phagocytic cell recognition, thereby disrupting phagocytosis and limiting the production of superoxide (82). P. aeruginosa can likewise produce a series of extracellular proteases (83) that inactivate the complement cascade (opsonin production) and inactivate cytokines (chemical signals) required for immune response activation. Extracellular toxins (e.g. exotoxins $\mathrm{A}, \mathrm{S}$ and $\mathrm{U}$ ) produced by $P$. aeruginosa also act to inhibit phagocytic activity by causing direct damage to the host cell. For example, exotoxin A disrupts the eukaryotic elongation factor 2 which directly affects host cell protein synthesis (40).

Like eukaryotic host cells, $P$. aeruginosa synthesises protective chaperone ("heat shock") proteins to deal with stressful environments, such as those experienced during phagocytosis. In addition to oxidative stress, phagocytosis also induces slight fluctuations in temperature (natural inflammation, increase blood supply, etc.). Heat 
shock proteins of $P$. aeruginosa are known to be transcriptionally upregulated under $\mathrm{H}_{2} \mathrm{O}_{2}$ exposure and other stressors (84). These proteins then act as molecular chaperones to protect protein folding mechanisms under stress (84). Furthermore, because heat shock proteins are highly conserved across bacterial species, they serve as major antigens for immune system microbial recognition (85). It has been hypothesised that microbe-specific heat shock determinants can then, through molecular mimicry $(86,87)$, become expressed on the cell surface of macrophages making the macrophage self-reactive (autoimmune) $(88,89)$.

Because $\mathrm{H}_{2} \mathrm{O}_{2}$ can cross the plasma membrane, it means that the macrophage is susceptible to damage as well, and it has antioxidant systems in place to contain the ROS that escape the phagolysosome. $P$. aeruginosa produces a bacteriocin called pyocin that interferes with these antioxidant systems by inhibiting catalases $(90,91)$ and inducing apoptosis $(92,93)$. At the same time, $P$. aeruginosa produces a pyocin immunity protein (94) which neutralises internal pyocin to prevent it from causing free radical damage within the bacterium $(95,96)$.

\subsubsection{Antioxidant systems of $P$. aeruginosa}

The antioxidant systems available to $P$. aeruginosa can be categorised as either nonenzymatic or enzymatic defence systems. Although most strategies to withstand oxidative stress involve an enzyme-based process, the production of alginate and biofilm is an example of a non-enzymatic ROS defence. Production of alginate not only stimulates the increase of other $P$. aeruginosa antioxidant systems $(97,98)$ but also protects against and scavenges for $\operatorname{ROS}(51,99,100)$. Some of the most significant and best studied enzymatic antioxidant systems are superoxide dismutase (SOD), peroxidase and catalase which detoxify $\mathrm{O}_{2}{ }^{\circ}, \mathrm{H}_{2} \mathrm{O}_{2}$, and peroxides respectively during phagocytosis $(101,102)$.

$P$. aeruginosa has two forms of SOD that scavenge for and catabolise $\mathrm{O}_{2}{ }^{\bullet}$ radicals, and which are active in the presence of manganese (MnSOD) or iron (FeSOD) (103-105). Although both FeSOD and MnSOD expression can be detected in most clinical $P$. aeruginosa isolates when grown under in vitro conditions, MnSOD only appears to be active in (i) the presence of oxygen and (ii) the absence of iron $(106,107)$. The absence of MnSOD in some clinical isolates suggests that MnSOD 
activity may not be essential for virulence $(107,108)$. FeSOD, on the other hand requires the presence of iron and has been shown to play a more important role in resistance to oxidative stress and pyocin biosynthesis (108).

Peroxidase and catalase enzymes directly catabolise cytotoxic $\mathrm{H}_{2} \mathrm{O}_{2}$ to water and $\mathrm{O}_{2}$ and, like FeSOD, require the presence of iron $(107,109,110)$. When challenged with $\mathrm{H}_{2} \mathrm{O}_{2}, P$. aeruginosa is known to use three catalase enzymes (KatA, KatB, and KatE) in the detoxification of $\mathrm{H}_{2} \mathrm{O}_{2}(109,110)$. KatA, the most potent of the three catalases, has been shown to be critical in the adaptation to $\mathrm{H}_{2} \mathrm{O}_{2}$ stress and for full virulence of $P$. aeruginosa (111). KatB and KatE, on the other hand do not appear to play a substantial role in $P$. aeruginosa in virulence (111). KatA of $P$. aeruginosa has well demonstrated stability compared to major catalases of other bacterial organisms and has been shown to be particularly important to $\mathrm{H}_{2} \mathrm{O}_{2}$ resistance at high concentrations $(112,113)$. Conversely, at low concentrations, catalase has been shown to act like a peroxidase and require the presence of a hydrogen donor to oxidise $\mathrm{H}_{2} \mathrm{O}_{2}$ (114). However, KatA has a very high $\mathrm{K}_{m}$ for $\mathrm{H}_{2} \mathrm{O}_{2}$ (44.7 mM; (110)); based on this we hypothesised that $P$. aeruginosa might possess additional systems for quenching $\mathrm{H}_{2} \mathrm{O}_{2}$ that have a greater affinity for this molecule, and that also contribute to $P$. aeruginosa virulence.

\subsubsection{1. $N A D(P) H-d e p e n d e n t ~ n i t r o-$ and quinone oxidoreductases (NQOR) - alternative bacterial antioxidant enzymes}

Nitro- and quinone- oxidoreductase enzymes (NQORs), also known as flavindependent quinone reductases, can be capable of simultaneous two-electron reduction of quinones leading to formation of quinol, avoiding the highly reactive semi-quinone intermediate (Figure 1.2, (115-117)). NQORs, although largely recognised for their membrane-bound role in respiration, can also be found in the cytosol of prokaryotes and eukaryotes alike (118) where their biological role is less understood. However, NQORs that are obligatory two-electron reducers are thought to contribute to oxidative protection by direct quinone-mediated quenching of $\mathrm{H}_{2} \mathrm{O}_{2}$ (119). 


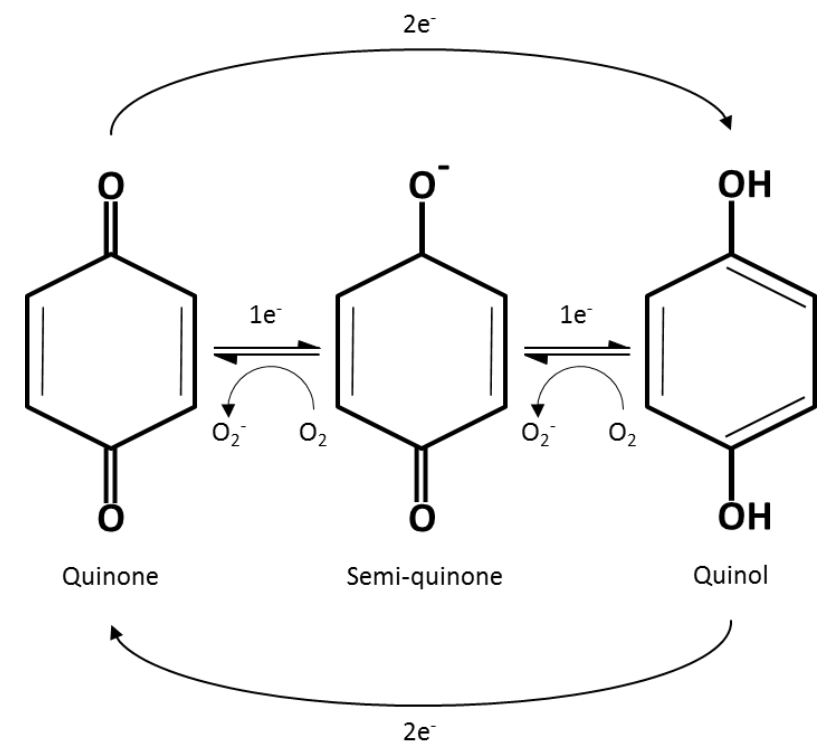

Figure 1.2. Schematic overview of quinone reduction

Single electron reduction of the parental quinone compound results in a highly reactive and unstable semi-quinone radical capable of acting on the parental quinone to produce superoxide. In the presence of a two-electron reduction step, the parental quinone is reduced to a stable quinol.

The soluble $\mathrm{NAD}(\mathrm{P}) \mathrm{H}$ quinone oxidoreductase enzyme of mammals, first described by Ernster et al in 1958 (NQO1, or DT-Diaphorase; (120)), is believed to have a primary role as an antioxidant (121). It has been extensively characterised as possessing the ability to detoxify thereby contributing to oxidative stress protection $(116,117,120,122)$. DT-diaphorase has also been shown to have the chemical flexibility to donate electrons to a broad range of substrates in addition to quinones $(115,123)$.

Nearly four decades after Ernster et al began to establish the mammalian quinone oxidoreductase as a potent antioxidant; prokaryotic enzymes exhibiting DTdiaphorase-like properties were identified. In the mid-1990s, the tryptophan repressor-binding protein, WrbaA (124), was shown to have sequence identity and structural homology with the mammalian protein $(125,126)$. Since then, several soluble bacterial quinone oxidoreductases have been identified and reported to display the biochemical properties necessary to perform simultaneous two electron reduction of quinones (Ackerley et al 2004 (127), Wang et al 2004 (128), Adams et al 2005 (129), Gonzalez et al 2005 (130), Akhtar et al 2006 (131), Patridge et al 2006 (132), and Guina et al 2007 (133)).The identification of NQORs in bacteria further suggests that NQORs may have a role in oxidative stress protection which will be explored further in Chapter 3. 


\subsubsection{NQOR metabolism of nitro-aromatic compounds}

Although highly promiscuous with regard to substrates that have been tested in vitro (e.g. chromate, quinone, and nitro-compounds; $(127,134,135))$, the above bacterial NQORs, like their mammalian DT-diaphorase counterparts, are believed to have a primary biological role of quinone metabolism. Even so, a number of these enzymes have been pursued for their ability to reduce nitro-aromatic anti-cancer prodrugs by the same simultaneous two electron transfer mechanism (136-139). This simultaneous two mechanism is important, as a large number of the prodrugs relevant to this field were originally developed as substrates for human 1-electron reductases, which can in analogous fashion to the 1-electron quinone reductases of Figure 1.2 - only reduce them through to their cytotoxic form in the absence of oxygen (i.e. under tumour hypoxia; (140). In contrast, the divalent bacterial NQORs can activate these prodrugs independent of the oxygen status of the cell, and are therefore of interest for gene therapy strategies that seek to target both aerobic and hypoxic tumour tissues.

Whilst this work primarily sought to characterise the role of NQORs as antioxidant enzymes in $P$. aeruginosa, the opportunity arose to investigate $P$. aeruginosa NQORs for their possible application in activation of nitro-aromatic prodrugs as well. Reductive prodrugs such as 5-aziridinyl-2,4-dinitrobenzamide (CB 1954), are relatively non-toxic in their administered form, but become highly toxic upon reduction $(136,141,142)$. The focus on activation of such compounds by bacterial NQORs stems from the observations that 1) although mammalian DT-diaphorase gene expression is known to be elevated in tumour cells it is almost entirely inactive with CB1954, and far less active with other promising nitro-aromatic prodrugs than many bacterial NQORs; and 2) the ubiquitous presence of DT-diaphorase in normal tissue would likely cause severe side effects if a prodrug that was a good substrate for this enzyme were to be administered systemically (143). Development of effective vector systems that specifically target tumour cells and can therefore be used to deliver therapeutic genes such as bacterial NQORs is of great interest $(139,140)$. This anticancer gene therapy strategy is generally known as gene directed enzyme prodrug therapy (GDEPT; $(140,144-147))$. The potential relevance of the $P$. aeruginosa NQORs to this strategy is explored further in Chapter 5. 


\subsection{Aims of this thesis}

Although NQORs have been proposed to play a role in bacterial virulence on the basis of biochemical and genetic evidence, a potential biological role for NQORs in $P$. aeruginosa antioxidant defence has yet to be determined; and given the need to better understand the mechanisms by which $P$. aeruginosa is able cause disease, we investigated the role of NQORs under oxidative stress as potential therapeutic targets. Additionally, because virulence involves a complex interplay between $P$. aeruginosa and immune system, we sought to examine the role of NQORs in the presence of murine macrophages to further examine a possible ROS-protective role for NQORs, as well as taking a more global view of the $P$. aeruginosa transcriptional response to challenge by activated macrophages. Finally, during the course of this thesis we had the opportunity to test the ability of the $P$. aeruginosa NQORs to activate a promising new prodrug for GDEPT. On the basis of some highly promising preliminary results this was pursued further and ultimately became a major aim of this study. The majority of the enzymes that were selected as the primary focus of this thesis have not previously been studied in $P$. aeruginosa in any context.

The primary goals of this project were:

1) To biochemically characterise NQORs of $P$. aeruginosa and assess the NQORs for their ability to protect against oxidative stress in vitro (Chapters 3 and 4).

2) To develop a method whereby NQORs of macrophage-engulfed $P$. aeruginosa can be assessed for their ability to protect against phagocytosis and subsequently, to apply this method for genome-wide expression analysis (Chapter 4).

3) To investigate the ability of $P$. aeruginosa NQORs to activate anti-tumour prodrugs for GDEPT (Chapter 5). 


\subsection{Flow chart: thesis overview}

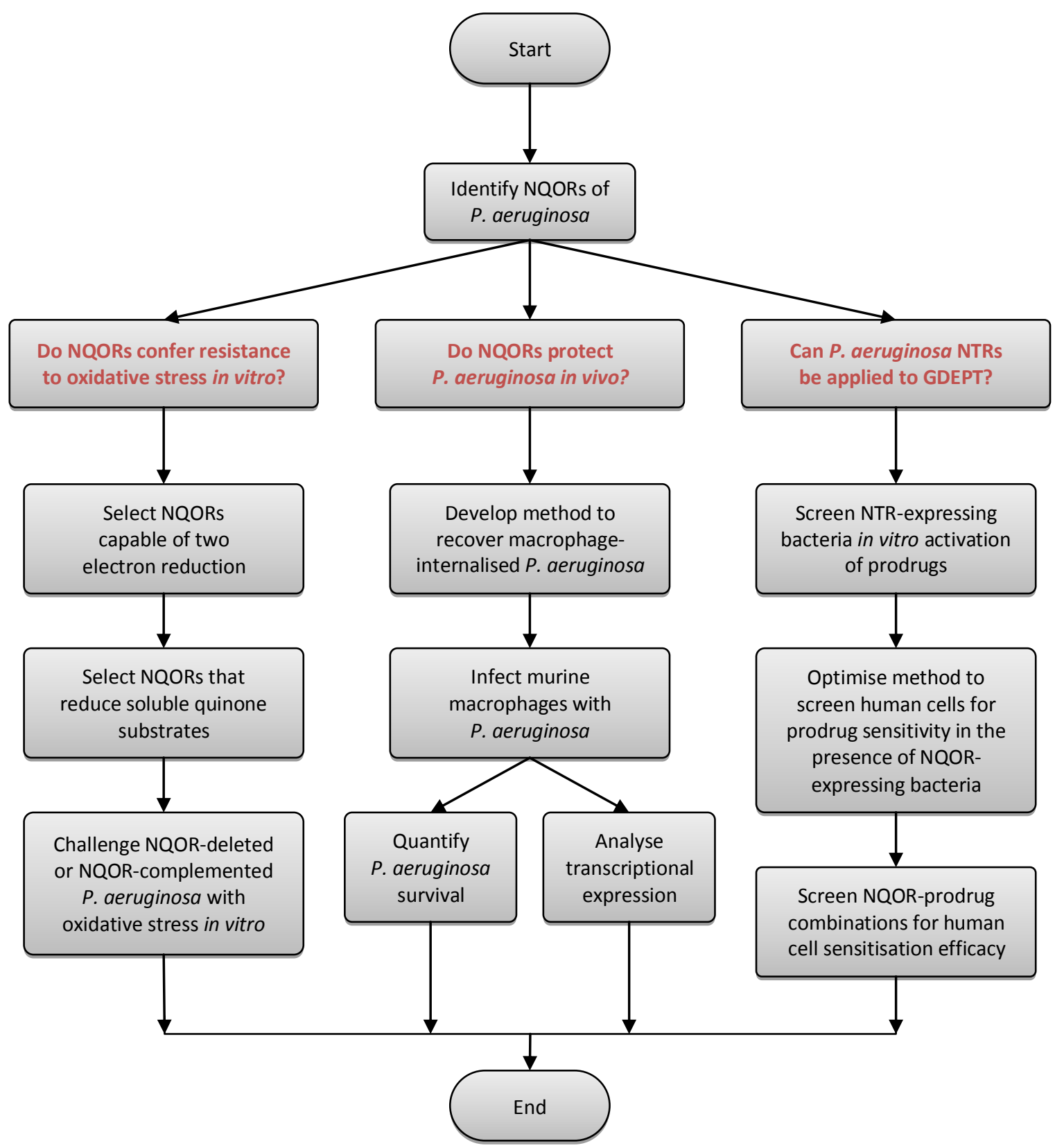




\section{Chapter 2: General Methods and Materials}

\subsection{Chemicals, enzymes, reagents, media}

All chemicals, reagents and media used in this study were obtained from Sigma-Aldrich (St. Louis. MO, USA) or Thermo Fisher Scientific (Waltham, MA, USA) unless otherwise stated. IPTG (isopropyl-D-thiogalactoside) and BioMix ${ }^{\mathrm{TM}}$ Red Mix were supplied by Bioline (London, UK). Restriction enzymes were supplied by New England Biolabs (NEB; Ipswich, MA, USA). T4 DNA ligase was supplied by Invitrogen (Carlsbad, CA, USA). Phusion $^{\mathrm{TM}}$ high-fidelity DNA polymerase was supplied by Finnzymes (Espoo, Finland).

\subsection{Oligonucleotide Primers}

Oligonucleotide primers used in this study were synthesized by Integrated DNA Technologies (IDT; Coralville, IA, USA). Lyophilized primers were resuspended to 100 $\mu \mathrm{M}$ in $1 \times \mathrm{TE} \mathrm{pH} 8.0$ (10 mM Tris-Cl pH 8.0, $0.1 \mathrm{mM}$ EDTA) and stored at $-20{ }^{\circ} \mathrm{C}$. For working stocks, aliquots were diluted in autoclaved, $0.22 \mu \mathrm{m}$ filter-sterilized distilled and deionised $\left(\mathrm{ddH}_{2} \mathrm{O}\right)$ water to $10 \mu \mathrm{M}$ final concentration.

Table 2.1.Oligonucleotide primers used in this study

Bold sequences indicate restriction sites. Underlined sequences indicate regions of homology used in recombination.

\begin{tabular}{ll}
\hline Primer name & Sequence (5' to 3') \\
\cline { 1 - 2 } Gene specific primers & \\
PA0853_Fwd & GGGGCATATGGTTGAAACCGCCAAGACC \\
PA0853_Rev & GGGGGTCGACCTAGACCTGGCTGCCGAGCT \\
PA0949_Fwd & GGGGCATATGTTGAGCAGTCCCTACATCCT \\
PA0949_Rev & GGGGGTCGACTCAACTCCCCAGCTTGCCGG \\
PA1204_Fwd & GGGGCATATGAGCGACGACATCAAG \\
PA1204_Rev & GGGGGTCGACTCAACCGCGCAGGCGGCGCA \\
PA1224_Fwd & GGGGCATATGAACGTACTCATCGTC \\
PA1224_Rev & GGGGCGGCCGCTCATTCGGC \\
PA1224_Rev_HindIII & GGGGAAGCTTTCATTCGGCCA \\
PA1225_Fwd & GGGGCATATGCATGCCCTGATCGTC \\
PA1225_Rev & GGGGGTCGACTCAGGCCTCCAGCGGCTGCG \\
PA1962_Fwd & GGGGCATATGAAACTTTTGCATATCGAT \\
PA1962_Rev & GGGGGTCGACTCAGGCCGCGGCGAACTGCCC \\
PA2357_Fwd & CCCCCATATGACCAGCCCCTTCAAA
\end{tabular}


PA2357_Rev
PA2580_Fwd
PA2580_Rev
PA2932_Fwd
PA2932_Rev
PA3208_Fwd
PA3208_Rev
PA3223_Fwd
PA3223_Rev
PA4236_Fwd
PA4236_Rev
PA4975_Fwd
PA4975_Rev
PA5190_Fwd
PA5190_Rev

Gene deletion primers

0949_KO_UL

0949_KO_LL

0949_KO_UR

0949_KO_LR

1204_KO_UL

1204_KO_LL

1204_KO_UR

1204_KO_LR

1224_KO_UL

1224_KO_LL

1224_KO_UR

1224_KO_LR

2580_KO_UL

2580_KO_LL

2580_KO_UR

2580_KO_LR

4236_KO_UL

4236_KO_LL

4236_KO_UR

4236_KO_LR

4975_KO_UL

4975_KO_LL

4975_KO_UR

4975_KO_LR
CCCGTCGACTCAGGCGATCTTCAACGG

CCCCATATGATGAAAAACATTCTCCTGC

CCCGTCGACTCAGCCGGCGC

CCCCATATGTCCAACCTGCTCCTCTCCCCGCTC

CCCCTCGAGGGGAATGCCAAGCACGGTGGCGGC

GGGGCATATGGAGGCTCTGACGCCT

GGGGTCGACTCAGCCCGGCCAGGCGCT

GGGGCATATGTCCCGTGTCCTGGTTATC

GGGGGTCGACTCACCGGAGCGTTGGCAGAGG

GGGGCATATGATGGAAGAGAAGACCCGCCT

GGGGGCGGCCGCTCAGTCCAGCTTCAGGCCGA

GGGGCATATGAACGTACTGATCGTC

GGGGGTCGACTCAGCGCGCCAGCGGCTGGA

GGGGCATATGCATATCGAAGACGCC

GGGGGTCGACTCAGAAGCGGTCGCGAATGA

CCCCTCTAGACCAGCAGCGTC

ACTGCTCAAGCGAGGATCTC

ICCTGGAGATCCTCGCTTGAGCAGTGGGAGTTGAAATGGCCCGCA

CCCCTCTAGACTGCATCGACG

GGGGTCTAGAATCGGAACGCCGCTTCGCCG

GTCGTCGCTCATGCCGTGCT

CGAGGAGCACGGCATGAGCGACGACCTGCGCGGTTGAATGCCTCG

GGGGTCTAGACAGGTCGGGATGATGGTCGA

GGGGTCTAGAATTCGCGTCGGCGCCAGCTC

GAGTACGTTCATGGCGGGTC

CCGGAGACCCGCCATGAACGTACTCCTGGCCGAATGACCCCGGGG

GGGGTCTAGACGCGCTTCGGCCTGGCCGAC

CCCCTCTAGACGGAGGCCATG

AATGTTTTTCATGGTGTTCC

TGTACGGAACACCATGAAAAACATTCGCGCCGGCTGAGTTCGACG

CCCCTCTAGACAGGTCCCAGT

GGGGTCTAGA GACAGCGTCGCCAACCGTCG

СТСТTССАТTTAСТСТСТСС

GTTGAGGAGAGAGTAAATGGAAGAGCTGGACTGATGGCCTGATGA

GGGGTCTAGA CCAACTGGGCCTTGAGGATG

CCCCTCTAGATCGCCGAGCGC

CAGTACGTTCATCGTGGTTT

ITCGGAAACCACGATGAACGTACTGCTGGCGCGCTGACCCGGGCG CCCCTCTAGAACGAGCGCTTC 
Sequencing and/or plasmid-specific primers

pMMBFwd

GGCTCGTATAATGTGTGG

pMMBRev

GACCGCTTCTGCGTTCTGAT

T7promoter

TAATACGACTCACTATAGGG

T7terminator

GCTAGTTATTGCTCAGCGG

M13Fwd

GTAAAACGACGGCCAG

M13Rev

CAGGAAACAGCTATGAC

pEX18GFwd

TGGGTAACGCCAGGGTTTTC

pEX18GRev

TGTGGAATTGTGAGCGGATA

\subsection{Bacterial strains (including clinical isolates) and plasmids}

Table 2.2. Bacterial strains used in this study

\begin{tabular}{|c|c|c|}
\hline Strain & Relevant characteristics & Source \\
\hline \multicolumn{3}{|c|}{ Escherichia coli strains } \\
\hline $\mathrm{DH} 5 \alpha \lambda$ pir & $\begin{array}{l}\text { F- endA1 gInV44 thi-1 recA1 relA1 gyrA96 } \\
\text { deoR nupG } \Phi 80 \mathrm{~d} / a c Z \Delta \mathrm{M} 15 \Delta(\text { lacZYA- } \\
\operatorname{argF)} \mathrm{U} 169, \text { hsdR17 }\left(\mathrm{r}_{\mathrm{K}}^{-} \mathrm{m}_{\mathrm{K}}^{+}\right), \lambda(\text { pir })\end{array}$ & Invitrogen \\
\hline S17-1 $\lambda$ pir & $\begin{array}{l}\text { TpR SmR recA, thi, pro, hsdR-M }{ }^{+} R P 4: \text { 2-Tc:Mu: } \\
\text { Km Tn7 } \lambda \text { (pir) }\end{array}$ & Lab stock \\
\hline BL21 & $\begin{array}{l}\mathrm{F}^{-} \text {ompT gal dcm lon hsdS }{ }_{B}\left(r_{B}^{-} m_{B}^{-}\right) \lambda(\text { DE3 [lacl } \\
\text { lacUV5-T7 gene1 ind1 sam7 nin5]) }\end{array}$ & Novagen \\
\hline SOS-R2 & ADA520 $\Delta n f s A \Delta n f s B \Delta t o l C$ & Prosser et al, 2010 (139) \\
\hline$\triangle 6 K O$ & $\begin{array}{l}\text { W3110 } \Delta n f s A \Delta n f s B \Delta a z o R \Delta y i e F \triangle y c a K \\
\Delta m d a B\end{array}$ & created by Claire Horvat \\
\hline$\triangle A B$ & ADA510 $\Delta n f s A \Delta n f s B$ & Prosser et al, 2010 (139) \\
\hline W3110 & Wild type Escherichia coli & Lab stock \\
\hline AKN98 & $\begin{array}{l}\text { Conjugative helper strain containing the } \\
\text { mobilizing plasmid pRK } 600 \text { carrying the } \\
\text { RP4/RK2 conjugation system }\end{array}$ & Lab stock \\
\hline \multicolumn{3}{|c|}{ Pseudomonas aeruginosa strains } \\
\hline PAO1 & Wild type genome sequenced strain & (148) \\
\hline ARL 1024 & Clinical sample: catheter, urine & $\mathrm{ESR}^{\mathrm{b}}$ \\
\hline ARL 1205 & Clinical sample: left thigh ${ }^{\mathrm{a}}$ & $\mathrm{ESR}^{\mathrm{b}}$ \\
\hline ARL001 1323 & Clinical sample: cystic fibrosis sputum & $\mathrm{ESR}^{\mathrm{b}}$ \\
\hline ARL 1079 & Clinical sample: tracheal aspirate ${ }^{a}$ & $\mathrm{ESR}^{\mathrm{b}}$ \\
\hline $\begin{array}{l}\text { ARL } 1236 \\
{ }^{\mathrm{a}} \text { from same pa } \\
{ }^{\mathrm{b}} \text { Environmenta }\end{array}$ & $\begin{array}{l}\text { Clinical sample: blood } \\
\text { tient } \\
\text { I Science and Research Culture Collection, Poriru }\end{array}$ & $\mathrm{ESR}^{\mathrm{b}}$ \\
\hline KT2440 & $\begin{array}{l}\text { Wild type genome sequenced Pseudomonas } \\
\text { putida strain }\end{array}$ & Lab stock \\
\hline \multicolumn{3}{|c|}{ Pseudomonas aeruginosa gene-deleted strains } \\
\hline$\Delta k a t A$ & $\mathrm{PAO} 1 \Delta k a t A$ & This study \\
\hline$\Delta 1204$ & $\mathrm{PAO} 1 \Delta 1204$ & This study \\
\hline$\Delta k a t A, \Delta 1204$ & $\mathrm{PAO} 1 \Delta k a t A, \Delta 1204$ & This study \\
\hline
\end{tabular}


Table 2.3. Plasmids used in this study

\begin{tabular}{|c|c|c|}
\hline Plasmid & Relevant characteristics & Source \\
\hline pEX18Gm & $\mathrm{Gm}^{\mathrm{R}}$, sacB. Suicide vector for gene deletion. & $\begin{array}{l}\text { Hoang et al, } 1998 \\
\text { (149) }\end{array}$ \\
\hline pET28a(+) & $\begin{array}{l}\text { Kan }^{R} \text {. Expression vector for } 6 \text { His-tagged } \\
\text { enzyme purification. } T 7 \text { promoter. }\end{array}$ & Novagen \\
\hline pUCX & $\begin{array}{l}\mathrm{Amp}^{\mathrm{R}} \text {. E. coli expression vector. tac promoter, } \\
\text { lac operator, } \mathrm{pET} 28 \mathrm{a}(+) \mathrm{RBS}\end{array}$ & $\begin{array}{l}\text { Prosser et al, } 2010 \\
\text { (139) }\end{array}$ \\
\hline pDM4 & $\mathrm{Chl}^{\mathrm{R}}$, sacB. Suicide vector for gene deletion. & $\begin{array}{l}\text { Milton et al, } 1996 \\
\text { (150) }\end{array}$ \\
\hline pSX & $\begin{array}{l}\mathrm{Gm}^{\mathrm{R} .} \text { pUCP22 backbone with expression } \\
\text { region from pUCX, allows IPTG regulated } \\
\text { expression of His-Tagged proteins in } \\
\text { Pseudomonas species. }\end{array}$ & Owen et al, 2011 (151) \\
\hline
\end{tabular}

\subsection{Bioinformatics}

Protein homology searches were carried out using the alignment search algorithm BLAST, hosted by the National Centre for Biotechnology Information (NCBI) protein database (http://blast.ncbi.nlm.nih.gov/; (152)) or the Pseudomonas Genome Database (http://www.pseudomonas.com/; (153)).

Sequence alignments were performed using the ClustalW2 Multiple Sequence Alignment (http://www.ebi.ac.uk/Tools/msa/clustalw2/; (154)) with input sequence data from the Pseudomonas Genome Database (3). Microarray data analysis was performed using BRB ArrayTools (155) in conjunction with DAVID Bioinformatics Resources 6.7 (http://david.abcc.ncifcrf.gov/; (156)).

\subsection{Bacterial growth and maintenance}

\subsubsection{Bacterial growth media}

All media used to support bacterial growth in this study was prepared using the appropriate volume of $\mathrm{ddH}_{2} \mathrm{O}$ unless otherwise stated. Sterilization was achieved either by autoclaving for $30 \mathrm{~min}$ at $121{ }^{\circ} \mathrm{C}$ or by sterile filtration using a $0.22 \mu \mathrm{m}$ filter (Millipore; Billerica, MA, USA) prior to use. Media were stored at room temperature (no supplements) or at $4{ }^{\circ} \mathrm{C}$ (supplemented or heat sensitive). 
2.5.1.1. Luria-Bertani (LB) Broth

\begin{tabular}{ll}
\hline Compound & Final Concentration \\
\hline Tryptone & $1 \%(\mathrm{w} / \mathrm{v})$ \\
Yeast extract & $0.5 \%(\mathrm{w} / \mathrm{v})$ \\
$\mathrm{NaCl}$ & $1 \%(\mathrm{w} / \mathrm{v})$ \\
\hline
\end{tabular}

2.5.1.2. TYM Broth

\begin{tabular}{ll}
\hline Compound & Final Concentration \\
\hline Tryptone & $2 \%(\mathrm{w} / \mathrm{v})$ \\
Yeast extract & $0.5 \%(\mathrm{w} / \mathrm{v})$ \\
$\mathrm{NaCl}$ & $100 \mathrm{mM}$ \\
$\mathrm{MgCl}_{2}{ }^{\mathrm{a}}$ & $10 \mathrm{mM}$ \\
${ }^{\mathrm{a}}$ Solution was added after autoclaving
\end{tabular}

\subsubsection{Solid growth media}

Solid media was prepared by adding molecular-grade agar to LB at $1.5 \%(\mathrm{w} / \mathrm{v})$ prior to sterilization by autoclaving for $30 \mathrm{~min}$ at $121{ }^{\circ} \mathrm{C}$. Media was allowed to cool to $50{ }^{\circ} \mathrm{C}$ prior to the addition of antibiotic supplements. Cooling media was then aseptically distributed to $90 \mathrm{~mm}$ diameter Petri dishes at $20 \mathrm{~mL}$ per plate and allowed to solidify at room temperature. All agar plates were stored at $4{ }^{\circ} \mathrm{C}$ and subsequently warmed to room temperature prior to use.

\subsubsection{Media supplements}

All stock solutions for antibiotic and other media supplements in this study were made up in the appropriate solvent to the indicated concentration and stored at $-20{ }^{\circ} \mathrm{C}$. Except for those compounds resuspended in absolute ethanol, all were filter sterilized using a $0.22 \mu \mathrm{m}$ filter. The final concentration used for routine growth or selection of $P$. aeruginosa and/or E. coli strains are listed below.

Table 2.4. Media Supplements

\begin{tabular}{lll|ll}
\hline Compound & Stock Solution & Solvent & P. aeruginosa & E. coli \\
\hline Ampicillin & $100 \mathrm{mg} / \mathrm{mL}$ & $\mathrm{ddH}_{2} \mathrm{O}$ & - & $100(\mu \mathrm{gg} / \mathrm{mL})$ \\
Kanamycin & $50 \mathrm{mg} / \mathrm{mL}$ & $\mathrm{ddH}_{2} \mathrm{O}$ & - & $50(\mu \mathrm{g} / \mathrm{mL})$ \\
Chloramphenicol & $34 \mathrm{mg} / \mathrm{mL}$ & ethanol & $200(\mu \mathrm{g} / \mathrm{mL})$ & $34(\mu \mathrm{g} / \mathrm{mL})$ \\
Gentamycin & $50 \mathrm{mg} / \mathrm{mL}$ & $\mathrm{ddH}_{2} \mathrm{O}$ & $100(\mu \mathrm{g} / \mathrm{mL})$ & $10(\mu \mathrm{g} / \mathrm{mL})$ \\
IPTG & $100 \mathrm{mg} / \mathrm{mL}$ & $\mathrm{ddH}_{2} \mathrm{O}$ & - & - \\
\hline
\end{tabular}




\subsubsection{Bacteria growth and storage}

Unless otherwise stated, bacterial cultures used in this study were prepared by inoculating LB medium (containing the appropriate supplements) from glycerol stocks; bacteria in liquid media containing $40 \%(\mathrm{w} / \mathrm{v})$ glycerol stored at $-80{ }^{\circ} \mathrm{C}$. Inoculated cultures were incubated at $37{ }^{\circ} \mathrm{C}$ under rotational agitation at 250 revolutions per minute (rpm). Bacteria on solid media were stored at $4{ }^{\circ} \mathrm{C}$ for up to 3 weeks.

\subsection{Routine molecular biology}

\subsubsection{Preparation of genomic DNA}

Bacterial genomic DNA (gDNA) was extracted from stationary-phase culture using the DNeasy ${ }^{\mathrm{TM}}$ Blood \& Tissue kit (Qiagen, GmbH, Germany) and prepared according to the manufacturer's specifications. Genomic DNA was stored at $-20{ }^{\circ} \mathrm{C}$.

\subsubsection{Miniprep}

Plasmid DNA was prepared according to the manufacturer's instructions using either Zyppy $^{\mathrm{TM}}$ Plasmid Miniprep kit (Zymo Research; Irvine, CA, USA) or Geneaid High-Speed Plasmid Mini Kit (Geneaid Biotech Ltd.; Sijhih City, Taiwan).

\subsubsection{PCR}

For amplification of all genes for cloning, Phusion ${ }^{\mathrm{TM}}$ high-fidelity polymerase was used. For all other PCRs, including colony screening, diagnostic and overlap PCR for gene knockout, BioMix ${ }^{\mathrm{TM}}$ Red was used. 
Table 2.5. PCR Reactions

\begin{tabular}{ll}
\hline Phusion $^{\text {TM }}$ Mixture & Volume per $20 \mu \mathrm{L}$ reaction \\
\hline $5 \times$ Phusion $^{\text {TM }} \mathrm{GC}$ buffer & $4 \mu \mathrm{L}$ \\
$10 \mathrm{mM}$ dNTPs & $0.4 \mu \mathrm{L}$ \\
$10 \mu \mathrm{M}$ primer 1 & $1 \mu \mathrm{L}$ \\
$10 \mu \mathrm{M}$ primer 2 & $1 \mu \mathrm{L}$ \\
Template gDNA & $1 \mu \mathrm{L}$ \\
DMSO & $0.6 \mu \mathrm{L}$ \\
$\mathrm{ddH}_{2} \mathrm{O}$ & Add to $20 \mu \mathrm{L}$ final volume \\
\hline & \\
\hline BioMix $^{\text {TM }}$ Red Mixture & Volume per $\mathbf{2 0} \mu \mathrm{L}$ reaction \\
\hline $2 \times$ BioMix $^{\mathrm{TM}}$ Master Mix & $10 \mu \mathrm{L}$ \\
$10 \mu \mathrm{M}$ primer 1 & $1 \mu \mathrm{L}$ \\
$10 \mu \mathrm{M}$ primer 2 & $1 \mu \mathrm{L}$ \\
Template DNA & $0.2-1 \mu \mathrm{L}$ \\
$\mathrm{DMSO}^{\mathrm{a}}$ & $1 \mu \mathrm{L}$ \\
$\mathrm{ddH}_{2} \mathrm{O}$ & $\mathrm{Add}$ to $20 \mu \mathrm{L}$ final volume \\
\hline
\end{tabular}

${ }^{\mathrm{a}}$ For colony PCR screens, a small amount of $E$. coli colony, picked directly from an agar plate with a sterile toothpick or pipette tip, was used as template DNA

Table 2.6. PCR Programmes

\begin{tabular}{|c|c|c|}
\hline Temperature $\left({ }^{\circ} \mathrm{C}\right)$ & Time & Cycles \\
\hline \multicolumn{3}{|l|}{ Phusion $^{\mathrm{TM}}$} \\
\hline 98 & $1 \mathrm{~min}$ & \\
\hline 98 & $30 \mathrm{sec}$ & \\
\hline $65^{a}$ & $30 \mathrm{sec}$ & 9 \\
\hline 72 & 30 sec per kb & \\
\hline 98 & $30 \mathrm{sec}$ & \\
\hline 55 & $30 \mathrm{sec}$ & $25-30$ \\
\hline 72 & 30 sec per kb & \\
\hline 72 & $5 \mathrm{~min}$ & \\
\hline 12 & Hold & \\
\hline \multicolumn{3}{|l|}{ BioMix ${ }^{T M}$ Red } \\
\hline 94 & $5 \mathrm{~min}$ & \\
\hline 94 & $30 \mathrm{sec}$ & \\
\hline $65^{a}$ & $30 \mathrm{sec}$ & 9 \\
\hline 72 & 1 min per kb & \\
\hline 94 & $30 \mathrm{sec}$ & \\
\hline 55 & $30 \mathrm{sec}$ & $25-30$ \\
\hline 72 & 1 min per kb & \\
\hline 72 & $5-10 \mathrm{~min}$ & \\
\hline 12 & Hold & \\
\hline
\end{tabular}




\subsubsection{PCR Purification}

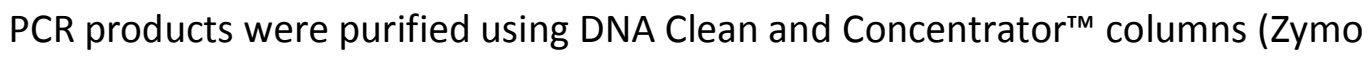
Research; Irvine, CA, USA) according to the manufacturer's instructions. Products were eluted using sterile-filtered $(0.22 \mu \mathrm{m}) \mathrm{dd}_{2} \mathrm{O}$ and stored at $-20^{\circ} \mathrm{C}$.

\subsubsection{Gel electrophoresis}

Qualitative assessment of DNA size and purity was performed by agarose gel electrophoresis ( $1 \%$ agarose $(\mathrm{w} / \mathrm{v})$ in $1 \mathrm{x}$ TAE buffer, $1 \mu \mathrm{g} / \mathrm{mL}$ ethidium bromide) run in 1x TAE buffer at 100-140 V for 30-60 min. HyperLadder ${ }^{\mathrm{TM}}$ (Bioline; London, UK) DNA marker was used for size comparison and DNA was visualized using ultra-violet light.

\subsubsection{Restriction enzyme (RE) digests}

To reduce supercoiling, DNA for restriction digests was incrementally heated from 23 ${ }^{\circ} \mathrm{C}$ to $70{ }^{\circ} \mathrm{C}$ for $15 \mathrm{~min}$ and then cooled to $37{ }^{\circ} \mathrm{C}$ prior to the addition of restriction enzyme and mixture components. Mixtures were then incubated at $37{ }^{\circ} \mathrm{C}$ for $6 \mathrm{~h}$ and subsequently heat inactivated at $65^{\circ} \mathrm{C}$ for $20 \mathrm{~min}$. Successful DNA digestion was confirmed by gel electrophoresis before being purified by DNA Clean and Concentrator ${ }^{\mathrm{TM}}$ kit (Zymo Research; Irvine, CA, USA) according to the manufacturer's instructions. Purified product was either used immediately or stored at $-20{ }^{\circ} \mathrm{C}$.

\section{Table 2.7. RE Reactions}

\begin{tabular}{ll}
\hline Solution & Volume per $20 \mu \mathrm{L}$ reaction \\
\hline 10x Restriction buffer & $2 \mu \mathrm{L}$ \\
20x Bovine Serum Albumin & $0.2 \mu \mathrm{L}$ \\
$\mathrm{RE} 1$ & $1 \mathrm{U} / \mu \mathrm{g}$ DNA \\
$\mathrm{RE} 2$ & $1 \mathrm{U} / \mu \mathrm{g}$ DNA (if required) \\
DNA & $50-1000 \mathrm{ng}$ \\
$\mathrm{ddH}_{2} \mathrm{O}$ & Add to $20 \mu \mathrm{L}$ final volume \\
\hline
\end{tabular}

\subsubsection{Ligation}

Ligation reactions were performed at room temperature overnight. Each individual reaction contained no more than $100 \mathrm{ng}$ total DNA at a concentration of $<10 \mathrm{ng}$ total 
DNA per $\mu \mathrm{L}$. Vector and insert DNA was combined using a molar ratio of 1:3 vector: insert. Products were then used directly to transform chemically competent $E$. coli.

Table 2.8. Ligation Reactions

\begin{tabular}{ll}
\hline Solution & Volume per $\mathbf{1 0} \mu \mathrm{L}$ reaction \\
\hline 5x Ligation buffer & $2 \mu \mathrm{L}$ \\
T4 DNA ligase & $1 \mathrm{U}$ \\
Digested vector DNA & $40-60 \mathrm{ng}$ \\
Digested insert DNA & $10-50 \mathrm{ng}$ \\
$\mathrm{ddH}_{2} \mathrm{O}$ & Add to $10 \mu \mathrm{L}$ final volume \\
\hline
\end{tabular}

\subsubsection{Bacterial Transformation}

\subsubsection{Preparation of chemically competent $E$. coli}

Overnight cultures of $E$. coli strains, inoculated from glycerol stocks, were grown in TYM broth to late-stationary phase then diluted to an optical density of $\mathrm{OD}_{600} 0.1$ in 50 $\mathrm{mL}$ fresh TYM broth. Cultures were grown at $37^{\circ} \mathrm{C}, 250 \mathrm{rpm}$ for $2-3 \mathrm{~h}$ until the $\mathrm{OD}_{600}$ reached between 0.5 and 1.0. Cells were then chilled on ice for $20 \mathrm{~min}$ and centrifuged at $4,000 \mathrm{rpm}$ for $10 \mathrm{~min}$ at $4{ }^{\circ} \mathrm{C}$. The cell pellet was resuspended in 1 volume ice-cold TFB I and left on ice for 2-3 h. Centrifugation was repeated and the cell pellet then resuspended in 0.1 volumes of TFB II and distributed to $100 \mu \mathrm{L}$ aliquots in sterile 1.5 $\mathrm{mL}$ microfuge tubes, snap frozen in a pre-chilled $-80{ }^{\circ} \mathrm{C}$ metal tube rack, and finally stored at $-80^{\circ} \mathrm{C}$.

Table 2.9. E. coli competent cell solutions

\begin{tabular}{ll}
\hline Compound & Final Concentration \\
\hline$T F B I$ & \\
Potassium acetate & $30 \mathrm{mM}$ \\
$\mathrm{MnCl}_{2}$ & $50 \mathrm{mM}$ \\
$\mathrm{CaCl}_{2}$ & $10 \mathrm{mM}$ \\
Glycerol & $15 \%(\mathrm{v} / \mathrm{v})$ \\
& \\
$T F B ~ I I$ & \\
$\mathrm{MOPS}, \mathrm{pH} 7.0$ & $10 \mathrm{mM}$ \\
$\mathrm{CaCl}$ & $75 \mathrm{mM}$ \\
$\mathrm{KCl}$ & $10 \mathrm{mM}$ \\
$\mathrm{Glycerol}$ & $15 \%(\mathrm{v} / \mathrm{v})$ \\
\hline
\end{tabular}




\subsubsection{E. coli transformation}

For each transformation reaction, one $100 \mu \mathrm{L}$ aliquot of chemically competent cells was thawed on ice for $5 \mathrm{~min}$. Plasmid DNA was added (no more than 0.1 the volume of competent cells being used) to the cells and the mixture was chilled on ice for $30 \mathrm{~min}$. Cells were then heat shocked at $42{ }^{\circ} \mathrm{C}$ for $90 \mathrm{sec}$ on a heating block, returned to ice for $5 \mathrm{~min}$, and recovered in $600 \mu \mathrm{L} \mathrm{LB}$ at $37{ }^{\circ} \mathrm{C}, 250 \mathrm{rpm}$ for $45 \mathrm{~min}$. Recovered cells were then spread onto solid media plates containing the necessary antibiotics for selection and incubated at $37{ }^{\circ} \mathrm{C}$ overnight until colonies became visible.

\subsubsection{Preparation of chemically competent $P$. aeruginosa}

Competent $P$. aeruginosa cells were made chemically competent by treatment with either sucrose or TG salts. These cells were used for transformation with complementation vectors.

\subsection{Sucrose method}

Overnight cultures of $P$. aeruginosa strains, inoculated from glycerol stock, were grown to late-stationary phase and diluted 1:10 in fresh LB. Cultures were allowed to continue growth at $37{ }^{\circ} \mathrm{C}, 250 \mathrm{rpm}$ until optical density reached $\mathrm{OD}_{600} 1.0$ at which point $6 \mathrm{~mL}$ aliquots of the cells were made in $15 \mathrm{~mL}$ conical tubes on ice. The bacteria were then pelleted by centrifugation at 5,000 rpm for 3 min and washed twice in $4 \mathrm{~mL}$ of room temperature $300 \mathrm{mM}$ sucrose. Competent cells were stored on ice (up to $1 \mathrm{~h}$ ) until ready for transformation.

\subsection{TG salts method}

$P$. aeruginosa cells to be made competent by treatment with TG salts were grown overnight in LB and diluted 1:10 in fresh LB. Upon returning to an optical density of $O D_{600} 1.0,1 \mathrm{~mL}$ aliquots of the culture were prepared in $1.5 \mathrm{~mL}$ microfuge tubes on ice. The cells were then pelleted at room temperature for $30 \mathrm{sec}, 10,000 \mathrm{rpm}$, the supernatant discarded, and the pellet resuspended in $1 \mathrm{~mL}$ ice-cold $100 \mathrm{mM} \mathrm{MgCl}$. 
Centrifugation was repeated and the cell pellet then resuspended in $1 \mathrm{~mL}$ ice-cold TG salts. The suspension was left to stand on ice for $10 \mathrm{~min}$, pelleted at 10,000 rpm for 30 sec, and resuspended in $200 \mu \mathrm{L}$ TG salts. Competent cells were stored on ice (up to $1 \mathrm{~h}$ ) until ready for transformation.

Table 2.10. $P$. aeruginosa competent cell solutions (TG salts)

\begin{tabular}{ll}
\hline Component & Final Concentration \\
\hline Glycerol & $10 \%(\mathrm{v} / \mathrm{v})$ \\
$\mathrm{MgCl}_{2}$ & $6 \mathrm{mM}$ \\
$\mathrm{CaCl}_{2}$ & $75 \mathrm{mM}$ \\
\hline
\end{tabular}

\subsubsection{Pseudomonas spp. Transformation}

For each aliquot of freshly prepared competent $P$. aeruginosa cells, $600 \mathrm{ng}$ of plasmid DNA was added and the mixture allowed to sit on ice for $15 \mathrm{~min}$ before being heat shocked at $37^{\circ} \mathrm{C}$ for $4 \mathrm{~min}$ in a water bath. Transformation mixtures were then recovered in $600 \mu \mathrm{L}$ fresh LB for $45 \mathrm{~min}$ at $37^{\circ} \mathrm{C}, 250 \mathrm{rpm}$. Recovered cells were spread onto solid media plates containing the necessary antibiotics for selection and incubated at $37{ }^{\circ} \mathrm{C}$ overnight until colonies became visible.

\subsubsection{DNA quantification}

Quantification of DNA in solution, including PCR products and purified plasmids, was performed using a NanoDrop ND-1000 spectrophotometer (Thermo Scientific), according to the manufacturer's instructions.

\subsubsection{Sequencing}

All DNA sequence confirmation in this study was carried out by Macrogen Inc. (Seoul, South Korea). 
2.7. SDS-PAGE

For qualitative assessment of protein products by SDS-PAGE, 15\% polyacrylamide gels (1 $\mathrm{mm}$ thickness) were prepared and run in the Bio-Rad Protean $\mathrm{II}^{\mathrm{TM}}$ apparatus. Each gel was constructed by loading $5 \mathrm{~mL}$ of $12 \%$ separating gel into the casting system and overlaying with $1 \mathrm{~mL}$ of $100 \%$ drum isopropanol. After the separating gel had set (30 $\mathrm{min})$, the isopropanol was removed and replaced with $1.5 \mathrm{~mL} 4 \%$ stacking gel. A multiwell comb was inserted into the gel cast and the stacking gel allowed to set ( $30 \mathrm{~min}$ ).

Samples run on SDS-PAGE were prepared in $3 \times$ SDS-loading buffer (total volume $30 \mu \mathrm{L}$ ) and boiled at $95{ }^{\circ} \mathrm{C}$ for $5 \mathrm{~min}$. Gels were run in $1 \times$ SDS-Run buffer at 200 $\checkmark$ for $45 \mathrm{~min}$. Protein bands in gels were stained by soaking in Coomassie blue stain solution for $30 \mathrm{~min}$. Visualization was achieved by removing the Coomassie by gentle agitation in destain solution.

Table 2.11. SDS-PAGE materials

\begin{tabular}{ll}
\hline Component & Final Concentration \\
\hline $15 \%$ separating gel & \\
$40 \%$ acrylamide solution & $36.5 \%(\mathrm{v} / \mathrm{v})$ \\
$2 \%$ Bis-acrylamide solution & $20.1 \%(\mathrm{v} / \mathrm{v})$ \\
Tris-Cl, pH8.8 & $375 \mathrm{mM}$ \\
SDS & $0.1 \%(\mathrm{w} / \mathrm{v})$ \\
APS $^{\mathrm{a}}$ & \\
TEMED $^{\mathrm{a}}$ & \\
& \\
$4 \%$ stacking gel & \\
$40 \%$ acrylamide solution & $9.6 \%(\mathrm{v} / \mathrm{v})$ \\
$2 \%$ Bis-acrylamide solution & $5.2 \%(\mathrm{v} / \mathrm{v})$ \\
Tris-Cl, pH6.8 & $125 \mathrm{mM}$ \\
SDS & $0.1 \%(\mathrm{w} / \mathrm{v})$ \\
APS & \\
TEMED & \\
& \\
$3 \times$ SDS loading buffer & \\
Tris-Cl, pH6.8 & \\
SDS & $150 \mathrm{mM}$ \\
Bromophenol blue & $6 \%(\mathrm{w} / \mathrm{v})$ \\
Glycerol & $0.3 \%(\mathrm{w} / \mathrm{v})$ \\
B-mercaptoethanol & $30 \%(\mathrm{v} / \mathrm{v})$ \\
& $300 \mathrm{mM}$ \\
1x SDS Running Buffer & \\
Glycine & $14.4 \mathrm{~g}$ \\
Tris & $3.03 \mathrm{~g}$ \\
SDS & $1 \mathrm{~g}$
\end{tabular}


Coomassie blue stain solution

Coomassie Brilliant Blue

Abs. ethanol

$450 \mathrm{~mL}$

Acetic acid

$100 \mathrm{~mL}$

Destain Solution

Methanol

$400 \mathrm{~mL}$

Acetic acid

$100 \mathrm{ml}$

${ }^{\mathrm{a}}$ Compound added immediately prior to use

\subsection{Purification of recombinant His6-tagged enzymes}

\subsubsection{E. coli enzyme expression and biochemical characterization}

E. coli BL21 cultures containing $\mathrm{pET} 28 \mathrm{a}(+)$ constructs were grown overnight to latestationary phase in $3 \mathrm{~mL} \mathrm{LB}$ at $37^{\circ} \mathrm{C}, 250 \mathrm{rpm}$. Cultures were then diluted to $\mathrm{OD}_{600} 0.1$ in $50 \mathrm{~mL} 1 \mathrm{M}$ sorbitol, $2.5 \mathrm{mM}$ betaine, LB and incubated at $18{ }^{\circ} \mathrm{C}, 200 \mathrm{rpm}$ until the $\mathrm{OD}_{600}$ reached 0.5 at which point enzyme expression was induced by addition of IPTG ( $0.5 \mathrm{mM}$ final concentration). Induced cultures were returned to incubation at $18{ }^{\circ} \mathrm{C}$, $200 \mathrm{rpm}$ for an additional $9 \mathrm{~h}$. Bacterial cultures were then placed on ice for $15 \mathrm{~min}$ before being collected by centrifugation for $15 \mathrm{~min}$ at $4,000 \mathrm{rpm}, 4{ }^{\circ} \mathrm{C}$. Cell pellets were either promptly processed or stored at $-80{ }^{\circ} \mathrm{C}$ until required for lysis.

\subsubsection{Cell lysis}

The lysates for the extraction of protein were prepared by either three passages through a French press in ice-cold 1x phosphate buffered saline (PBS) or by the commercially available BugBuster ${ }^{\mathrm{TM}}$ protein-extraction reagent (Novagen; Merck, Darmstadt, Germany) according to the manufacturer's instructions.

Table 2.12. PBS (pH 7.4)

\begin{tabular}{ll}
\hline Component & Final Concentration \\
\hline $\mathrm{NaCl}$ & $1.45 \mathrm{M}$ \\
$\mathrm{Na}_{2} \mathrm{HPO}_{4}-12 \mathrm{H}_{2} \mathrm{O}$ & $20 \mathrm{mM}$ \\
$\mathrm{NaH}_{2} \mathrm{PO}_{4}-2 \mathrm{H}_{2} \mathrm{O}$ & $2 \mathrm{mM}$ \\
\hline
\end{tabular}




\subsubsection{Protein purification and storage}

Soluble protein fractions for purification were collected by centrifugation at 13,000 $\mathrm{rpm}, 4{ }^{\circ} \mathrm{C}$ for $15 \mathrm{~min}$ and the aqueous layer transferred to a fresh $15 \mathrm{~mL}$ conical tube on ice. Recombinant His6-tagged NQORs were then purified by nickel-affinity chromatography using the His $\bullet$ Bind Kit (Novagen) and following the manufacturer's instructions. Eluted protein was collected in $1.5 \mathrm{~mL}$ microfuge tubes on ice and incubated with $100 \mathrm{mM}$ FMN cofactor at $4{ }^{\circ} \mathrm{C}$ overnight. Purified proteins were then desalted using a $5 \mathrm{~mL} \mathrm{HiTrap}{ }^{\text {TM }}$ Desalting column (GE Healthcare, Chalfont St. Giles, UK) into $40 \mathrm{mM}$ Tris- $\mathrm{Cl}$, pH 7.0 according to the manufacturer's instructions. Samples were added to $40 \%$ glycerol $(\mathrm{v} / \mathrm{v})$ final concentration and stored at $-20^{\circ} \mathrm{C}$ indefinitely.

\subsubsection{Quantification}

Quantification of purified protein was performed by using the $\mathrm{Dc}^{\mathrm{TM}}$ protein assay kit (Bio-Rad) according to the manufacturer's instructions. Comparative protein standards of bovine serum albumin (BSA) were used for standard curve calculations.

\subsubsection{In vitro enzyme kinetics}

\subsection{Quinone substrate specificity}

Bacterial NAD(P)H oxidoreductase activity in the presence of quinone substrates was measured spectrophotometrically, following diminishing NADPH absorbance at $340 \mathrm{~nm}$ using a Helios UV-Vis spectrophotometer. One $\mathrm{ml}$ reaction mixtures consisted of 25$250 \mu \mathrm{M}$ quinone substrate, $0.1-5.0 \mu \mathrm{g}$ of purified recombinant NQOR in $50 \mathrm{mM}$ Tris-Cl buffer pH7.4 and were initiated by the addition of $250 \mathrm{mM}$ NADPH. Benzoquinone was added from $10 \mathrm{mM}$ stock in double-distilled water, menadione from $10 \mathrm{mM}$ stock dissolved in ethanol, and coenzyme Q1 from a $200 \mathrm{mM}$ stock in N,Ndimethylformamide that was subsequently diluted to a $10 \mathrm{mM}$ working stock in ethanol. For steady-state kinetic analysis, non-linear regression and Michaelis-Menten curve fitting was performed using GraphPad ${ }^{\mathrm{TM}}$ Prism 5 (GraphPad Software, La Jolla California USA). 


\subsection{Cytochrome $c$ trap}

In order to measure simultaneous two electron transfer by NQORs during benzoquinone reduction, the substrate cytochrome $c$ was used as a trap for semiquinone generation as it can only be reduced by univalent electron transfer. The reactive mixture consisted of $0.1-5.0 \mu \mathrm{g}$ of purified NQOR proteins or control lipoyl dehydrogenase from Clostridium kluyveri (Sigma) and $75 \mu \mathrm{M}$ cytochrome $c$ from horse heart (Sigma) in $50 \mathrm{mM}$ Tris- $\mathrm{Cl}$ pH 6.0. The reaction was activated by addition of 250 $\mu \mathrm{M} N A D(P) H$ and monitored at $550 \mathrm{~nm}$ for increased absorbance indicating reduction of the cytochrome $c$ substrate.

\subsection{Determination of SN 26438 extinction coefficients}

$100 \mu \mathrm{M}$ SN 26438 in 10 mM Tris-Cl pH 7.0, 4 \% DMSO was scanned for total absorbance between 325 and $500 \mathrm{~nm}$ wavelength, with a $1 \mathrm{~cm}$ path length, using a Helios y UV-Vis spectrophotometer (Thermo-Fisher). The same concentration of SN 26438 was then incubated with $300 \mu \mathrm{M}$ NADPH and $\sim 1 \mu$ g purified recombinant NfsA (this enzyme has innate $\mathrm{NAD}(\mathrm{P}) \mathrm{H}$ oxidase activity, so will convert any residual $\mathrm{NADPH}$ to $\mathrm{NADP}^{+}$thus eliminating the absorbance background from NADPH at $\left.\mathrm{OD}_{340}\right)$. This reaction was left to run for 20 min until NADPH oxidation had terminated as measured by decrease in. This reaction was then scanned for total absorbance as above. The wavelength at which the difference in absorbance between parent and reduced substrate was greatest was chosen for determination of the extinction coefficient $(\varepsilon)$. This was calculated by using Beer-Lambert Law adjusting for substrate concentration, so that the final constant was expressed in units of $\mathrm{M}^{-1} \mathrm{~cm}^{-1}$.

\subsection{In vitro purified NQORs kinetics with prodrug}

Steady-state enzyme kinetics with purified enzymes were measured as reported previously by Prosser et al (139) with minor modifications. Briefly, prodrug reduction was monitored spectrophotometrically at $420 \mathrm{~nm}$ for CB1954 $\left(\varepsilon=1200 \mathrm{M}^{-1} \mathrm{~cm}^{-1}\right)$ and $340 \mathrm{~nm}$ for SN $26438\left(\varepsilon=4770 \mathrm{M}^{-1} \mathrm{~cm}^{-1}\right)$. Reactions were performed in a total of $60 \mu \mathrm{l}$ reactions in UVettes (Eppendorf, Hamburg, Germany) containing reaction buffer (10 
$\mathrm{mM}$ Tris- $\mathrm{Cl}$ ( $\mathrm{pH}$ 7.0), 4\% DMSO, $0.25 \mathrm{mM} \mathrm{NAD(P)H}$ and varying concentrations of CB1954 (0 $\mu \mathrm{M}-2400 \mu \mathrm{M})$ or SN $26438(0 \mu \mathrm{M}-100 \mu \mathrm{M})$. Six $\mu$ l of the appropriate purified enzyme dilution was added to $54 \mu$ of reaction buffer and changes in absorbance were measured at $10 \mathrm{~s}$ intervals over $30 \mathrm{~s}$. For calculation of $\mathrm{K}_{\mathrm{m}}$ and $\mathrm{k}_{\mathrm{cat}}$ values, substrate concentrations were varied from $\sim 0.2 \times$ to $5 \times \mathrm{Km}$. Non-linear regression analyses and Michaelis-Menten curve fitting were performed using GraphPad Prism (Graph-pad software, Inc).

\subsection{Gene-deletion}

A two-step overlap PCR was used to generate a truncated NQOR gene to be integrated to host bacteria genome by a recombination event during bacterial conjugation. Using a modified touchdown protocol, KO PCR I, sequence $500-1000$ bp upstream and downstream of the target sequence was amplified, including the first and last three codons of the NQOR gene sequence. The downstream forward primer (UR primer) contained ( $5^{\prime}$ to $\left.3^{\prime}\right) 20$ nucleotides homologous to the $3^{\prime}$-end of the upstream sequence. The upstream forward primer (UL primer) and the downstream reverse primer (LR primer) were both flanked by Xbal restriction sites for cloning purposes. In the second-stage overlap KO PCR II, purified upstream and downstream PCR products from KO PCR I were added to a primer-less PCR reaction. At a lowered annealing temperature of $55^{\circ} \mathrm{C}$, the two homologous regions were allowed to selfanneal for three cycles before adding the upstream forward (UL) and downstream reverse (LR) primers to amplify the complete truncated NQOR gene sequence. Truncated gene sizes were confirmed by gel electrophoresis. 
A

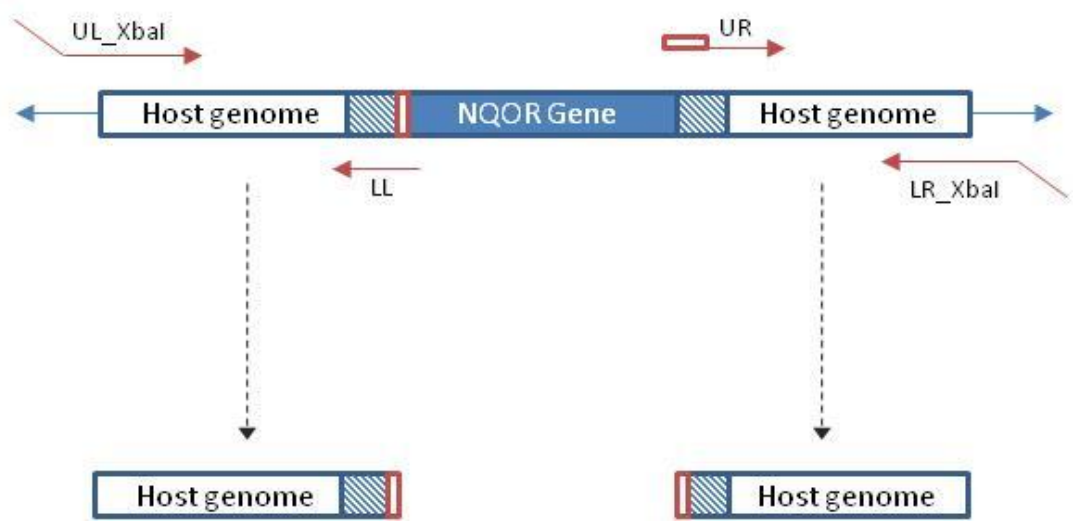

B

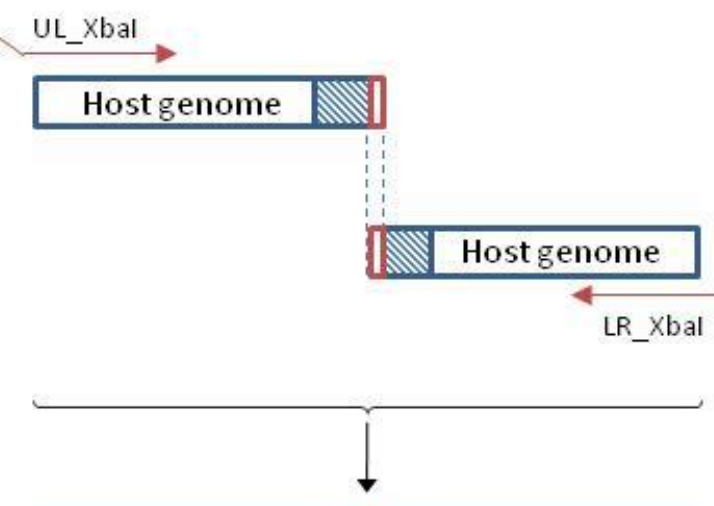

Host genome $\mathbb{N} / \mathbb{N}$ Host genome

Figure 2.1. Schematic overview of KO PCR I and II

(A) Amplification of regions upstream and downstream of the target gene to be truncated. (B) Selfannealing of upstream and downstream products in a primer-less PCR prior to amplification of the full length construct using gene primers.

The truncated NQOR gene was cloned into either the pDM4 or pEX18Gm $P$. aeruginosa replication vector and transformed into competent DH5alpha $\lambda$ pir E.coli. Tri-parental conjugation was then used to integrate the smaller gene form into host $P$. aeruginosa strain. In summary, competent host recipient (e.g. wild type $P$. aeruginosa) was combined with the donor strain (DH5 containing replication vector) and helper strain (ANK98 containing pRK600 mobilization vector carrying the RP4/RK2 conjugation system).

Donor and helper strains were inoculated from glycerol stock and grown $16 \mathrm{~h}$ in nutrient broth (containing the appropriate antibiotic) at $37{ }^{\circ} \mathrm{C}, 250 \mathrm{rpm}$. The recipient strain (PAO1) was grown $16 \mathrm{~h}$ in antibiotic-free nutrient broth supplemented with $0.4 \%$ 
$\mathrm{KNO}_{3}$ at $42{ }^{\circ} \mathrm{C}$ under static conditions. Each log-phase liquid culture was then diluted 10 -fold and returned to shaking incubation at $37{ }^{\circ} \mathrm{C}$ until $\mathrm{OD}_{600} 1.0$ was reached $(\sim 2-3$ h). Helper and donor strains were let to settle at room temperature for $15 \mathrm{~min}$ while the recipient was heat shocked at $50{ }^{\circ} \mathrm{C}$ for $4 \mathrm{~min}$ and settled on ice until required.

The tri-parental conjugation mixture $(0.75 \mathrm{~mL}$ recipient, $0.5 \mathrm{ml}$ donor, $0.2 \mathrm{ml}$ helper) was combined by gentle inversion and centrifuged briefly $(10,000 \mathrm{rpm}$ for 30 sec) at which point the supernatent was discarded and the bacterial pellet resuspended in $50 \mu \mathrm{L} \mathrm{LB}$ (no antibiotic). The suspension was then gently added to dry nutrient agar plate an incubated for $16 \mathrm{~h}$ at $37^{\circ} \mathrm{C}$ to allow for conjugation. The conjugation culture was then scraped from the agar and resuspended in $2 \mathrm{~mL}$ PBS at room temperature. Dilutions of the conjugation culture were seeded onto two nutrient agar plates containing a) no antibiotic as control or b) chloramphenicol (100 ng/mL), kanamycin $(20 \mathrm{ng} / \mathrm{mL})$ for pDM4 vectors and gentamycin (200 ng/ $\mu \mathrm{L})$, kanamycin (20 $\mathrm{ng} / \mathrm{\mu L}$ ) for $\mathrm{pEX} 18 \mathrm{Gm}$ vectors. These plates were then incubated at $37{ }^{\circ} \mathrm{C}$ for $16-24 \mathrm{~h}$ to select for primary integration of the truncated gene into the host genome. The initial integration step was selected on plates containing $100 \mu \mathrm{g} / \mathrm{mL} \mathrm{Chl} \mathrm{and} 20 \mu \mathrm{g} / \mathrm{mL} \mathrm{Kan}$ presence of the mutagenic plasmid confirmed by colony PCR.

The second recombination event was then forced by growing primary integrants overnight in nutrient broth supplemented with $5 \%$ sucrose $(w / v)$ incubated at $37{ }^{\circ} \mathrm{C}, 250 \mathrm{rpm}$. Serial dilutions were plated on solid medium containing $10 \% \mathrm{w} / \mathrm{v}$ sucrose and incubated at $37{ }^{\circ} \mathrm{C}$ until colonies appeared. After development, 20-40 colonies were patched onto LB medium with and without $100 \mu \mathrm{g} / \mathrm{mL} \mathrm{Chl}$ and $20 \mu \mathrm{g} / \mathrm{mL}$ Kan. Colonies which had reverted to $\mathrm{Chl}$ sensitivity were taken to be double crossover events and were confirmed by PCR. 


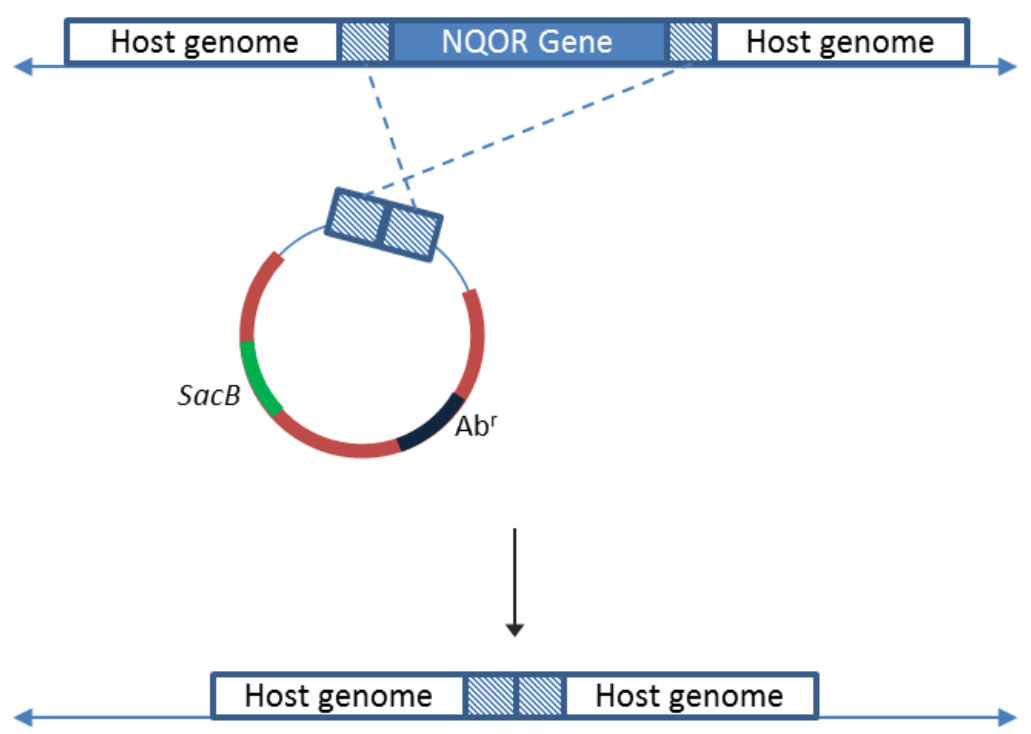

Figure 2.2. Schematic overview of primary and secondary recombinant integration Primary integration is selected for by the acquisition of antibiotic resistance. After incubation with sucrose, recombinants that have lost the $s a c B$-containing vector and completed full integration of the truncated NQOR are selected for by loss of antibiotic resistance.

\subsection{0. $\mathrm{H}_{2} \mathrm{O}_{2}$ challenge and Amplex ${ }^{\circledR}$ Red hydrogen peroxide assay}

Three $\mathrm{mL}$ overnight cultures were diluted to $\mathrm{OD}_{600} 0.1$ in fresh $\mathrm{LB}$ containing the appropriate antibiotic supplements, dispensed at $200 \mu$ l per well in a 96 well-plate, and allowed to recover for $45 \mathrm{~min}, 37^{\circ} \mathrm{C}, 250 \mathrm{rpm}$. At this point, time zero, the $\mathrm{OD}_{600}$ was measured. Serial dilutions of $100-0.1 \mathrm{mM} \mathrm{H}_{2} \mathrm{O}_{2}$ (final concentration) were added to the bacterial cultures to a final volume of $120 \mu \mathrm{L}$. The cultures were returned to incubation and the $\mathrm{OD}_{600}$ recorded every $60 \mathrm{~min}$ for $9 \mathrm{~h}$. Bacterial strain viability was measured as a percentage of growth relative to the unchallenged replicate.

For the Amplex ${ }^{\circledR}$ Red hydrogen peroxide assay, to measure quenching of $\mathrm{H}_{2} \mathrm{O}_{2}$ by $P$. aeruginosa strains, cultures were prepared as above. At each $60 \mathrm{~min} \mathrm{OD}_{600}$ measurement, $50 \mu \mathrm{L}$ of bacterial culture was removed and mixed 1:1 with Amplex ${ }^{\circledR}$ Red reaction mix (10 mM Amplex Red reagent, $10 \mathrm{U} / \mathrm{mL}$ horseradish peroxidase, $1 \mathrm{x}$ reaction buffer). Reactions were developed at room temperature for $30 \mathrm{~min}$ protected from light. Using an EnSpire ${ }^{\mathrm{TM}} 2300$ Multilabel Reader (PerkinElmer, Waltham, MA, USA) microplate reader equipped for excitation in the range of 530-560 nm and fluorescence emission detection at $\sim 590 \mathrm{~nm}, \mathrm{H}_{2} \mathrm{O}_{2}$ concentrations were calculated using a standard curve of $\mathrm{H}_{2} \mathrm{O}_{2}$ serial dilution controls in the same reaction mix. 


\subsection{Cell culture}

The established macrophage-like cell lines, RAW264.7 and J774.1, were generous gifts from the Malaghan Institute for Medical Research (MIMR; Wellington, NZ). HCT-116, a colorectal carcinoma cell line was a gift from the Auckland Cancer Society Research Centre, New Zealand. All cell lines in this study were maintained as described below.

Table 2.13. Mammalian strains used in this study

\begin{tabular}{lll}
\hline Strain & Organism \& Characteristics & Source \\
\hline J774A.1 & Murine, BALB/CN derived adherent macrophage & MIMR $^{\mathrm{a}}$ \\
RAW-264.7 & Murine, BALB/C derived adherent macrophage & $\mathrm{MIMR}^{\mathrm{a}}$ \\
HCT-116 & Human, colorectal carcinoma adherent epithelium & ACSRC $^{\mathrm{b}}$ \\
\hline
\end{tabular}

${ }^{a}$ Malaghan Institute for Medical Research

${ }^{\mathrm{b}}$ Auckland Cancer Society Research Centre

\subsubsection{Long term storage}

All cell lines used in this study were stored in aliquots of $2 \times 10^{6}$ cells per $1 \mathrm{~mL}$ freezing solution containing heat inactivated and filter sterilized Foetal Calf Serum (FCS), and $10 \%$ (v/v) DMSO. Aliquots were kept in 1.8 mL Nunc Cryo Tubes ${ }^{\mathrm{TM}}$ (Nunc GmbH \& Co. KG ; Germany) and stored long term in liquid nitrogen.

\subsubsection{Culture initiation}

Frozen liquid nitrogen stocks were revived by pre-warming $10 \mathrm{~mL}$ of complete culture media (CMEM; 1x Penicillin-streptomycin, 25 mM HEPES Buffer solution, 10\% FCS in basal media Gibco ${ }^{\circledR} \mathrm{DMEM}$; Invitrogen) to $37{ }^{\circ} \mathrm{C}$ in a water bath for $30 \mathrm{~min}$. The cryo vial of frozen cells was quickly thawed in $37{ }^{\circ} \mathrm{C}$ water and immediately transferred to the pre-warmed CMEM and centrifuged for $4 \mathrm{~min}$ at $400 \mathrm{xg}$, room temperature. The DMSO-containing supernatant was discarded and the cell pellet resuspended in fresh 7 $\mathrm{mL}$ CMEM and seeded into a CELLSTAR ${ }^{\circledR} 25 \mathrm{~cm}^{2}$ cell culture flask and incubated for 72 h at $37{ }^{\circ} \mathrm{C}, 5 \% \mathrm{CO}_{2}$. 


\subsubsection{Propagation and maintenance}

Routine cell line cultures were maintained every 72 hours as follows:

Nutrient-exhausted media was aseptically removed from the cellular monolayer. The monolayer was then rinsed with 1x Dubelco's Phosphate Buffered Saline (DPBS). The monolayer was then mechanically disrupted (RAW-264.7, J774A.1) or lifted using 0.05\% trypsin-EDTA (HCT-116). Cell suspensions were then transferred to a sterile 15 $\mathrm{mL}$ conical tube, centrifuged at $400 \mathrm{x} g$ for $4 \mathrm{~min}$, and then resuspended in fresh complete media before seeding into a new CELLSTAR $^{\circledR} 75 \mathrm{~cm}^{2}$ cell culture flask at $1 \times 10^{6}$ cells per $15 \mathrm{~mL}$ CMEM.

\subsection{Reactive oxygen release by RAW-264.7 macrophages}

RAW-264.7 macrophages were seeded at $8.4 \times 10^{3}$ cells per well in a 96 well microtitre plate. Approximately $48 \mathrm{~h}$ later positive control cells were treated with $10 \mu \mathrm{M} \mathrm{H}_{2} \mathrm{O}_{2}$ for $2 \mathrm{~h}$. Untreated and $\mathrm{H}_{2} \mathrm{O}_{2}$ treated cells washed twice in PBS and then incubated in the dark in $10 \mu \mathrm{M} \mathrm{CM}-\mathrm{H} 2 \mathrm{DCFDA}, \mathrm{PBS}$ at $37^{\circ} \mathrm{C}, 5 \% \mathrm{CO}_{2}$ for $30 \mathrm{~min}$ to load the dye (N.B. Untreated cells not loaded with dye were used as a negative control to examine cellular autofluorescence.). Wild type $P$. aeruginosa at MOI 50, MOI 100 or $200 \mathrm{ng} / 100$ $\mu \mathrm{L}$ LPS was applied to the dye-loaded RAW-264.7 cells in PBS and then incubation continued for $\sim 8 \mathrm{~h}$. Fluorescence was measured at 30-60 min intervals using an EnSpire $^{\mathrm{TM}} 2300$ Multilabel Reader (PerkinElmer, Waltham, MA, USA) microplate reader equipped for excitation at $495 \mathrm{~nm}$ and fluorescence emission detection at $520 \mathrm{~nm}$.

\subsection{Griess reaction}

To measure the release of the effector molecule, nitric oxide (NO), by macrophages, a Greiss reaction was used to measure the more stable NO breakdown product nitrite in a 96-well microtitre plate format. RAW264.7 macrophages were seeded at $8.4 \times 10^{3}$ cells per well and applied with MOI 100 P. aeruginosa, $8 \mu \mathrm{g}$ lipopolysaccharide (LPS), or $8 \mu \mathrm{g}$ LPS $+100 \mathrm{U} / \mathrm{mL}$ interferon-gamma including media only controls in triplicate. 50 $\mu \mathrm{L}$ of supernatants were removed at time intervals $0-11 \mathrm{~h}$ and added to a new 96well microtitre plate. Griess solutions $A$ and B were mixed in equal volumes and $50 \mu \mathrm{L}$ 
added to each well, final volume of $100 \mu \mathrm{L}$, and measured spectrophotometrically at absorbance $570 \mathrm{~nm}$. Nitrite concentration was determined by a standard curve prepared with sodium nitrite standards $(500 \mu \mathrm{M}-0 \mu \mathrm{M})$ in culture media and calculated using GraphPad Prism (Graph-pad software, Inc).

\subsection{Flow cytometry}

Bacteria cultures for flow cytometric analysis were washed 3 times in $1 x$ PBS to remove all traces of nutrient media and transferred to a round-bottom $5 \mathrm{~mL}$ polystyrene tube (BD Falcon, BD Biosciences; USA). The washed bacteria were then collected by centrifugation at $4000 \mathrm{x}$ for 4 minutes at room temperature and the supernatant removed. Cell pellets were then resuspended by vortex in $500 \mu \mathrm{L}$ normal saline viability staining solution containing $20 \mu \mathrm{g} / \mathrm{mL}$ propidium iodide, $1 \mathrm{x}$ SYTO ${ }^{\circledR} \mathrm{BC}$ and $1 \mathrm{x}$ microsphere standards (Bacteria Counting Kit, Molecular Probes, Invitrogen). Stained cells were incubated in the dark for 15 min before being processed on a FACSCanto II flow cytometer (BD Biosciences). Analysis was performed using FlowJo 7.6.1 software (Tree Star, Inc.).

\subsubsection{Live $P$. aeruginosa population analysis by flow cytometry - a gating} strategy for quantification

To gauge the survivability of $P$. aeruginosa using the FACSCanto II flow cytometer (BD Biosciences), all samples were first normalised against single stained and unstained controls to set collection parameters of threshold, mode, and voltage (Figure A4.1). For all analyses, forward scatter (FSC) and side scatter (SSC) were set log mode. Initial cellular debris discrimination was achieved by triggering events on SSC at a threshold of 2.0. Further event discrimination is described below. FSC and SSC voltages were set to $380-410$. $\mathrm{FL} 1$ (SYTO ${ }^{\circledR} \mathrm{BC}$ ) and FL2 (PI) channels were also set to log mode with voltages at 310-410. Acquisition-derived fluorescence compensation, as determined by the unstained and single-stained controls, was set at FL1-FL2 $=7.50 \%$ and FL2-FL1 $=$ $2.10 \%$. Minor adjustments to compensation post-collection were achieved using FlowJo v7.6.1 Compensation Editor Wizard. 


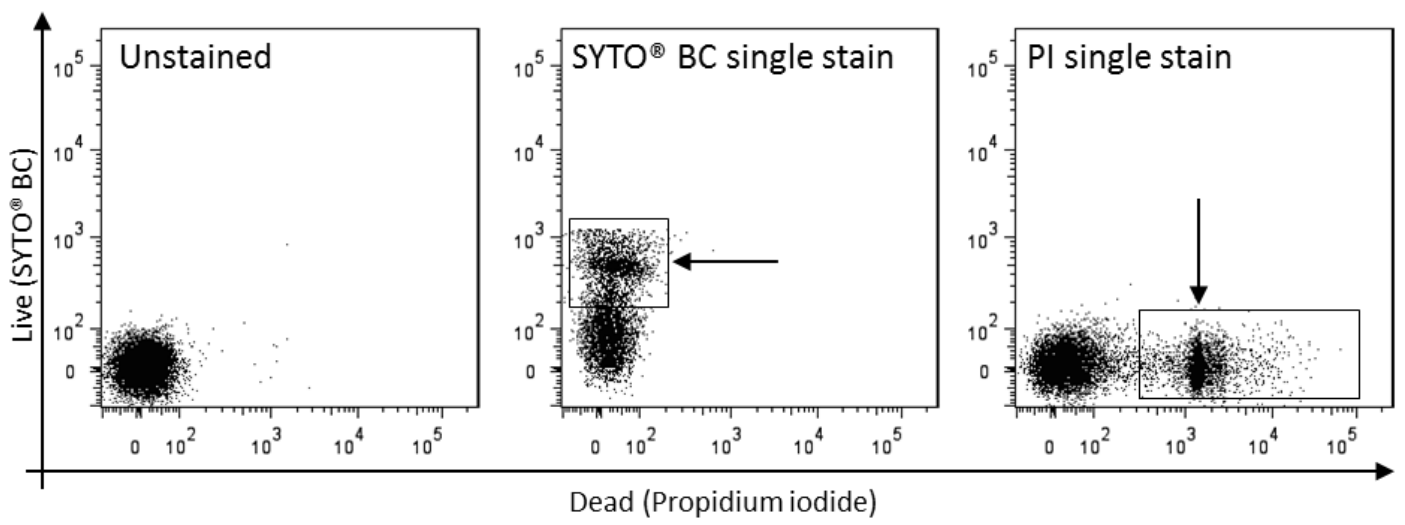

Figure 2.3. $P$. aeruginosa flow cytometry controls

$P$. aeruginosa cultures grown overnight in LB and washed in PBS were either left untreated, stained only with SYTO ${ }^{\circledR} \mathrm{BC}(1: 1000)$, or only with $\mathrm{PI}(1: 20)$. All staining solutions prepared in $0.9 \% \mathrm{NaCl}$, were stained for $15 \mathrm{~min}$ at room temperature in the dark as described in Chapter 4. Samples were then run on FACSCanto II flow cytometer (BD Biosciences) at the medium collection setting ( $60 \mu \mathrm{l} / \mathrm{min}$ ) having an acquisition rate of $\sim 1000$ events/second.

To quantify live (SYTO ${ }^{\circledR} \mathrm{BC}$ ) events, as represented on a dot plot cytogram, fluorescent bacterial signals were first differentiated from Bacteria Counting Kit microsphere bead standards by size using FSC and SSC (Figure A4.2a). The laser noise was then removed by excluding double negative events on $\mathrm{FL} 1\left(\mathrm{SYTO}^{\circledR} \mathrm{BC}\right)$ versus $\mathrm{FL} 2$ (PI) plots therefore selecting all bacteria-only signals (Figure A4.2b). Because the cell sorting determined that SYTO ${ }^{\circledR} \mathrm{BC}+\mathrm{PI}+$ populations were viable, total "live" populations for quantification were determined by gating all $\mathrm{SYTO}^{\circledR} \mathrm{BC}+$ events of all bacteria-only signals on a histogram (Figure A4.2c). 


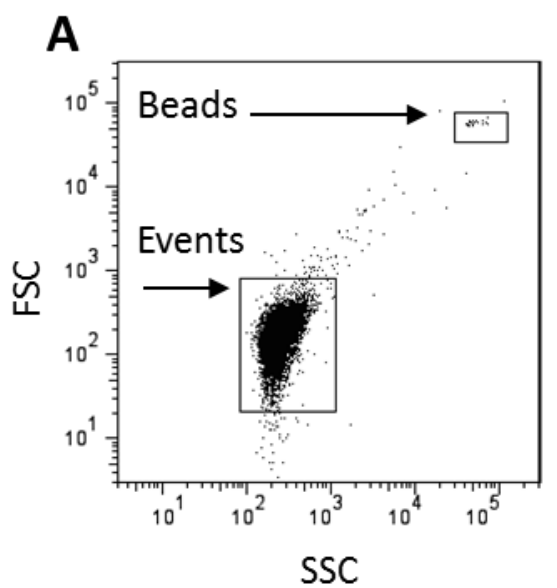

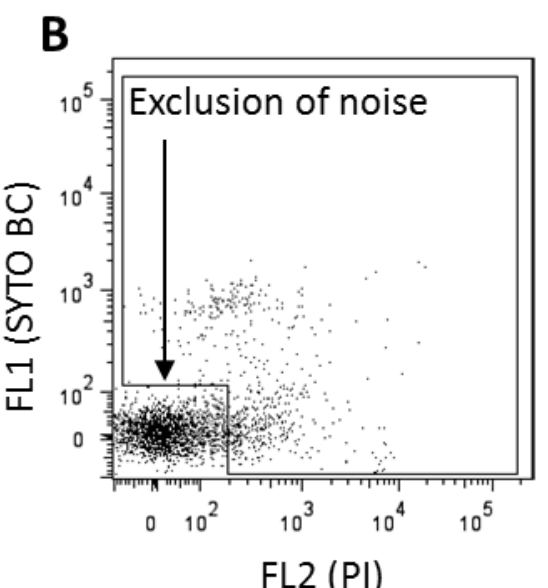

$\mathrm{FL} 2(\mathrm{PI})$

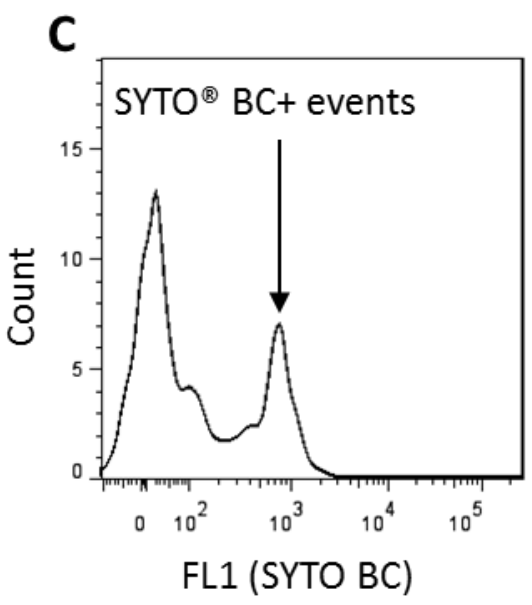

\section{Figure 2.4. $P$. aeruginosa event selection for quantification}

(A) P. aeruginosa stained with the flow cytometry staining solution as described in Chapter 4, were differentiated from Bacteria Counting Kit bead standards using FSC vs. SSC in log mode. (B) False (laser noise) events were then excluded by gating FL1 vs. FL2. (C) All true bacterial flow signals were then displayed in a histogram of SYTO ${ }^{\circledR} \mathrm{BC}+$ events.

The ratio of $\mathrm{SYTO}^{\circledR} \mathrm{BC}+$ bacterial signals (Figure $\mathrm{A} 4.2 \mathrm{c}$ ) to bead signals (Figure A4.2a) was then calculated and adjusted for dilution to accurately quantify the number of bacteria present.

$\frac{\text { Bacteria signals }}{\text { Bead signals }} \times$ Dilution factor $=$ Bacteria per $\mathrm{mL}$




\subsection{Confocal Microscopy}

Both bacterial and mammalian cell cultures were visualized using the laser-scanning confocal microscope FlowView ${ }^{\circledR}$ FV1000 (Olympus; USA). Images were analysed using Olympus FV10-ASW Viewer Ver.2.0b.

\subsubsection{Pure bacterial cultures}

Washed and pelleted bacteria in $5 \mathrm{~mL}$ polystyrene tubes were resuspended in $50 \mu \mathrm{L}$ normal saline viability staining solution (minus microsphere standards) and developed in the dark for $15 \mathrm{~min}$. As mounted on pre-cleaned $2 \mathrm{~mm}$ microscope slide, $5 \mu \mathrm{L}$ of stained cells were mixed 1:1 with $4 \%$ paraformaldehyde in normal saline. Samples were excited sequentially at $473 \mathrm{~nm}$ and $559 \mathrm{~nm}$ for viability on FlowView ${ }^{\circledR}$ FV1000.

\subsubsection{Mammalian cell monolayers}

For the visualization of $P$. aeruginosa uptake by murine macrophage cells, pure bacterial cultures were washed and prepared as above but stained only with $2 \mu \mathrm{L}$ SYTO ${ }^{\circledR} \mathrm{BC}$ in $50 \mu \mathrm{L}$ normal saline. Murine macrophage cell cultures were prepared by seeding $7.5 \times 10^{5}$ macrophages per $3 \mathrm{~mL}$ Gibco ${ }^{\circledR}$ DMEM (Dubelco's Modified Eagle Medium) culture media free of phenol red indicator (Invitrogen) supplemented with $10 \%$ foetal calf serum, 0.25 mM HEPES in a $35 \mathrm{~mm}$ glass-bottom FluoroDish ${ }^{\text {TM }}$ cell culture dish (World Precision Instruments, Inc.; Sarasota, Florida, USA). Cellular monolayers were washed twice with fresh, room temperature media and infected with stained $P$. aeruginosa as described in Chapter 4. Post-infection monolayers were gently washed with room temperature $1 x$ PBS and stained with the counterstain Image-iT ${ }^{\mathrm{TM}}$ LIVE Plasma Membrane and Nuclear Labelling Kit (Molecular Probes, Invitrogen) according to the manufacturer's instructions and imaged as above. 


\subsection{RNA Extraction and Purification}

All samples in the preparation of purified bacterial RNA for microarray analysis were treated with one millilitre of Trizol $^{\circledR}$ (Invitrogen) per $\mathrm{cm}^{2}$ culture surface. Trizol ${ }^{\circledR}$ treated samples were stored at $-80^{\circ} \mathrm{C}$ overnight before being thawed at room temperature for RNA extraction. Room temperature chloroform was added at $0.2 \mathrm{~mL}$ per $1 \mathrm{~mL}$ Trizol ${ }^{\circledR}$. The extraction solution was then shaken, incubated at room temperature for 3 minutes, and centrifuged at $4000 \mathrm{x}$ for $15 \mathrm{~min}$. The aqueous layer was collected to a new RNase-free tube and 2 volumes of room temperature absolute ethanol was added while gently vortexing. The ethanol mixture was then applied to an RNeasy Mini Kit spin column (QIAGEN; Germany) by centrifugation at 13,000 rpm in a 2 $\mathrm{mL}$ collection tube. The column was washed with $350 \mu \mathrm{l}$ Wash Buffer RW1, centrifuged for 15 seconds at 13,000 rpm, washed with $500 \mu \mathrm{l}$ Wash Buffer RPE and centrifuged for another 15 seconds at 13,000 rpm. After discarding the flow through, the column was washed for a second time with $500 \mu$ l Wash Buffer RPE and centrifuged for 2 minutes at 13,000 rpm. The washed column was then placed in a new collection tube and centrifuged for an additional 1 minute at 13,000 rpm to completely dry the RNA and remove traces of ethanol. The column was then transferred to an RNase-free $1.5 \mathrm{~mL}$ microfuge tube. RNA was eluted from the column by twice applying $30 \mu$ l of RNase-free $\mathrm{H}_{2} \mathrm{O}$ (for microarray application) or RNase-free 1x TE buffer (for enrichment). Internal PAO1 RNA was eluted in TE buffer ( $\mathrm{pH} 7.4$ ) for prokaryotic RNA enrichment using Ambion ${ }^{\circledR}$ MICROBEnrich ${ }^{\text {TM }}$ Kit (Life Technologies - Applied Biosystems/Ambion).

\subsubsection{Prokaryotic RNA Enrichment}

Bacterial RNA samples harvested from host macrophage cultures were enriched for using the Ambion ${ }^{\circledR}$ MICROBEnrich ${ }^{\mathrm{TM}}$ Kit (Life Technologies - Applied Biosystems/Ambion; Austin, TX, USA) according to manufacturer's specifications. MICROBEnrich ${ }^{\mathrm{TM}}$ uses magnetic separation to remove the 18S rRNA, 28S rRNA, and polyadenylated mRNA of eukaryotic tissue. Final enriched Internal PAO1 RNA was resuspended in $25 \mu \mathrm{L}$ RNase-free water for direct microarray application. For each RNA preparation, an aliquot of $1.5 \mu \mathrm{L}$ was removed for quality control. 


\subsubsection{RNA Quality Control}

The Agilent 2100 Bioanalyzer and Agilent RNA 6000 Nano Kit (Agilent Technologies; Santa Clara, CA, USA) were provided by Environmental Sciences and Research (ESR; Porirua, NZ) and used to assess the quality of purified RNA according to manufacturer's specifications.

\subsection{Microarray}

The GeneChip ${ }^{\circledR}$ Pseudomonas aeruginosa Genome Arrays (Affymetrix Inc., Santa Clara, CA, USA) were prepared and processed at according manufacturer's specifications by Liam Williams at the Centre for Genomics and Proteomics at the University of Auckland, New Zealand.

\subsubsection{Chips}

"The GeneChip ${ }^{\circledR}$ P. aeruginosa Genome Array contains probe sets for over 5,500 ORFs from the PAO1 strain of P. aeruginosa, 199 probe sets corresponding to 100 intergenic sequences, and 117 additional genes from $P$. aeruginosa strains other than PAO1. During the design process, the $P$. aeruginosa sequences were pruned against the GeneChip Human Genome U95 Genome Array, facilitating experiments on the $P$. aeruginosa samples isolated from human tissues. The $P$. aeruginosa Genome Array includes probe sets to intergenic sequences which may lead to the identification of new genes. In addition to sequences from the $P$. aeruginosa strain PAO1, the array also includes unique sequences from other strains to broaden the research possibilities with the array. Genes encoding serological determinants and pathogenicity islands 4 are included on the array and have proven useful in genotyping $P$. aeruginosa strains isolated from human infections originating from different tissues" (157).

\subsubsection{Preparation and processing}

cDNA preparation and hybridisation was performed by the Centre for Genomics, Proteomics and Metabolomics (CGPM) at the University of Auckland. Processing and 
scanning was completed according to the manufacturer's recommendations (www.affymetrix.com/support/technical/manual/expression_manual.affx).

\subsubsection{Data analysis}

Raw data normalisation and initial analysis was performed by Donia MacartneyCoxson, at Environmental Science and Research, using Bioconductor package V2.9 with R V2.14 (open source software, http://www.bioconductor.org/). Analysis was made by fitting data to a linear regression. The initial hypotheses were for differences in sample: Internal vs Control, External vs Control and Internal vs External. Many probes reached significance at an adjusted (for multiple testing using the Benjamini and Hochberg (method to control false discovery rate) $p$ value of $<0.05$. Further analysis RMA (robust multi-array chip averaging) as instigated in the Affy and Oligo Bioconductor packages uses quantile normalisation. This method was introduced by Bolstad et al 2003 and is one of the most commonly used methods. The method aims to give each chip the same empirical distribution, and brings the quantiles for each chip together. As RMA (with quantile normalisation) is often the method used in the literature, subsequent analyses used this method and all defaults background=True $(\mathrm{rma})$, normalisation $=$ True (quantile), pmcorrect $($ probe specific correction) $=$ pmonly and summary.method (summarisation of probe level data for a probe set) = median polish.

\subsection{SOS assay}

Individual wells of 96-well microtitre plates, containing $150 \mu \mathrm{l}$ LB amended with 100 $\mathrm{mg} / \mathrm{mL}$ ampicillin and $0.4 \%$ glucose, were inoculated with SOS-R2 strains expressing candidate nitroreductases in pUCX, together with a pUCX (empty plasmid) control. Cultures were incubated for $16.5 \mathrm{~h}$ at $30{ }^{\circ} \mathrm{C}$ with shaking at $200 \mathrm{rpm}$. Fifteen $\mu \mathrm{l}$ of overnight cultures were transferred to individual wells of a 96 -well microtitre plate containing fresh $200 \mu \mathrm{l}$ of LB amended with $100 \mathrm{mg} / \mathrm{mL}$ ampicillin, $0.05 \mathrm{mM}$ IPTG and $0.4 \%$ glucose (LB-AIG) per well for a final volume of $215 \mu \mathrm{L}$. Cells were grown at $30^{\circ} \mathrm{C}$, $200 \mathrm{rpm}$ for $3 \mathrm{~h}$, at which point they were diluted 1:1 in LB-AIG containing $20 \mu \mathrm{M}$ CB1954 or $5 \mu \mathrm{M}$ SN26438 in the final volume of $200 \mu \mathrm{L}$. Cells were then challenged for 
$3.5 \mathrm{~h}$ at $30{ }^{\circ} \mathrm{C}, 200 \mathrm{rpm}$. At this point the $\mathrm{OD}_{600}$ for each well was recorded and $20 \mu \mathrm{l}$ from each well were added to $130 \mu \mathrm{l}$ ZOB buffer (Table 2.13.) in a new 96-well microtitre plate for $\beta$-galactosidase analysis. Reactions were then incubated at $37{ }^{\circ} \mathrm{C}$ for $25 \mathrm{~min}$, or until the yellow colour of $o$-nitrophenyl- $\beta$-D-galactopyranoside (ONPG) substrate was clearly visible. The assay was terminated by addition of $50 \mu$ of $1 \mathrm{M}$ $\mathrm{Na}_{2} \mathrm{CO}_{3}$ and the $\mathrm{OD}_{420}$ and $\mathrm{OD}_{550}$ were recorded. Miller units were calculated using the modified Miller equation described by Prosser et al (139).

\section{Table 2.14. SOS buffers}

\begin{tabular}{|c|c|}
\hline Component & Final Concentration \\
\hline \multicolumn{2}{|l|}{1 M Sodium phosphate buffer $p H 7.0$} \\
\hline $\mathrm{Na}_{2} \mathrm{HPO}_{4}$ & $0.577 \mathrm{M}$ \\
\hline $\mathrm{NaH}_{2} \mathrm{PO}_{4}$ & $0.423 \mathrm{M}$ \\
\hline \multicolumn{2}{|l|}{ Z-buffer } \\
\hline $\mathrm{Na}_{2} \mathrm{HPO}_{4}$ & $0.126 \mathrm{M}$ \\
\hline $\mathrm{NaH}_{2} \mathrm{PO}_{4}$ & $0.126 \mathrm{M}$ \\
\hline $\mathrm{MgSO}_{4}$ & $2 \mathrm{mM}$ \\
\hline $\mathrm{MnSO}_{4}$ & $0.4 \mathrm{mM}$ \\
\hline CTAB & $399 \mathrm{mg} / \mathrm{mL}$ \\
\hline Sodium deoxycholate & $199.5 \mathrm{mg} / \mathrm{mL}$ \\
\hline B-mercaptoethanol ${ }^{\mathrm{a}}$ & $0.174 \mathrm{M}$ \\
\hline \multicolumn{2}{|l|}{ T-base } \\
\hline$\left(\mathrm{NH}_{4}\right)_{2} \mathrm{SO}_{4}$ & $15.1 \mathrm{mM}$ \\
\hline $\mathrm{K}_{2} \mathrm{HPO}_{4}$ & $0.08 \mathrm{M}$ \\
\hline $\mathrm{KH}_{2} \mathrm{PO}_{4}$ & $0.044 \mathrm{M}$ \\
\hline Sodium citrate & $1 \mathrm{~g} / \mathrm{L}$ \\
\hline o-nitrophenyl-ß-D-galactopyranoside ${ }^{a}$ & $8 \mathrm{mg} / \mathrm{mL}$ \\
\hline \multicolumn{2}{|l|}{ ZOB buffer } \\
\hline T-base & 1 part \\
\hline Z-buffer & 4 part \\
\hline
\end{tabular}


2.19. Sensitisation of HCT-116 cells to CB1954 and SN 26438 using E. coli expressing candidate NQORs

HCT-116 colorectal carcinoma cells (American Type Culture Collection, Manassas, VA, USA) were seeded into 96-well microtitre plate wells at $5 \times 10^{3}$ per $100 \mu \mathrm{L}$ of culture media [CMEM (Gibco, Invitrogen Corporation, Grand Island, NY, USA) supplemented with 5\% FCS and $10 \mathrm{mM}$ HEPES (Gibco)] and incubated at $37{ }^{\circ} \mathrm{C}, 5 \% \mathrm{CO}_{2}$ for $48 \mathrm{~h}$. E. coli $\triangle 6 K O$ and SOS-R2 strains expressing candidate NQORs from plasmid pUCX were prepared by inoculating $150 \mu \mathrm{L} L B+100 \mu \mathrm{g} / \mathrm{mL}$ amp $+0.4 \%(\mathrm{w} / \mathrm{v})$ glucose and incubating $16.5 \mathrm{~h}$ at $30^{\circ} \mathrm{C}, 200 \mathrm{rpm} .15 \mu \mathrm{L}$ of overnight cultures were then used to inoculate $200 \mu \mathrm{L}$ fresh culture media amended with $100 \mu \mathrm{g} / \mathrm{mL}$ amp + $0.05 \mathrm{mM}$ IPTG + $0.4 \%(\mathrm{w} / \mathrm{v})$ glucose (CMEM-AIG). NQORs-expressing E. coli were then grown at $30{ }^{\circ} \mathrm{C}$, $200 \mathrm{rpm}$ for an additional $3 \mathrm{~h}$. Meanwhile dilutions of CB1954 (200 $\mu \mathrm{M}-0 \mu \mathrm{M})$ and SN $26438(10 \mu \mathrm{M}-0 \mu \mathrm{M})$ were prepared in CMEM-AIG. Day cultures of E. coli $\triangle 6 K O$ and SOS-R2 strains expressing NQORs were mixed 1:1 with prodrug dilutions (SN 26438 and CB1954 respectively) in a final volume of $210 \mu \mathrm{L}$. HCT-116 monolayers were then washed twice with PBS. $100 \mu \mathrm{L}$ of E. coli:prodrug mixtures were applied to the washed $\mathrm{HCT}-116$ monolayer and incubated $4 \mathrm{~h}$ at $37{ }^{\circ} \mathrm{C}, 5 \% \mathrm{CO}_{2}$, after which the bacteria were removed from the HCT-116 monolayer by washing four times in PBS. The HCT-116 cells were then allowed to recover at $37{ }^{\circ} \mathrm{C}, 5 \% \mathrm{CO}_{2}$ for $48 \mathrm{~h}$ in culture media amended with $100 \mu \mathrm{g} / \mathrm{mL}$ chloramphenicol. $20 \mu \mathrm{L}$ of CellTiter $96^{\circledR} \mathrm{AQ}_{\text {ueous }}$ One Solution (Promega) was added to the cell culture and incubated for $2 \mathrm{~h}$ at $37^{\circ} \mathrm{C}, 5 \% \mathrm{CO}_{2}$. The $\mathrm{OD}_{490}$ was recorded to measure formazan levels, indicative of the number of respiring HCT-116 cells present in the culture. The $I C_{50}$ values were then calculated by comparing $\mathrm{OD}_{490}$ of the challenged cells with the unchallenged cells using GraphPad Prism (Graph-pad software, Inc). 


\section{Chapter 3: Characterisation of the antioxidant role of $\boldsymbol{P}$. aeruginosa NQORs}

\subsection{Introduction}

During the course of $P$. aeruginosa infections the bacterium encounters many forms of oxidative stress, with a major constituent being the highly reactive $\mathrm{H}_{2} \mathrm{O}_{2}$. P. aeruginosa, like most aerobes (including mammals), possess the potent antioxidant defence enzymes SOD and catalase. The $P$. aeruginosa-specific catalase, KatA, has previously been described as an $\mathrm{H}_{2} \mathrm{O}_{2}$-quenching virulence factor (111); and like SOD enzymes, is able to scavenge for and reduce ROS. Together, the bacterial SOD and catalase enzymes are thought to be the first line of defence when it comes to oxidative stress, a challenge $P$. aeruginosa must overcome at all stages of infection. The phagocytederived oxidative burst, and primary source of concentrated ROS exposure, can be considered to occur in two overlapping stages whereby the host phagocyte (i) initially produces superoxide through the NADH oxidase, succeeded then by (ii) sequential one-electron reductions of the superoxide radical to form hydrogen peroxide (158). SOD enzymes in general have a high affinity for the superoxide radical (159) and the superoxide reduction creates the $\mathrm{H}_{2} \mathrm{O}_{2}$ substrate that catalase is highly efficient at degrading into water and $\mathrm{O}_{2}$ (Figure $3.1 ;(160)$ ). Therefore, SOD and catalase have complementary activities and act in tandem to protect the bacterium (161).

$$
\begin{aligned}
& \text { Reaction (i) } \mathrm{O}_{2}^{\bullet-}+\mathrm{O}_{2}^{\bullet-}+2 \mathrm{H}^{+} \stackrel{\mathrm{SOD}}{\longrightarrow} \mathrm{O}_{2}+\mathrm{H}_{2} \mathrm{O}_{2} \\
& \text { Reaction (ii) } \quad \mathrm{H}_{2} \mathrm{O}_{2}+\mathrm{H}_{2} \mathrm{O}_{2} \stackrel{\text { KatA }}{\longrightarrow} 2 \mathrm{H}_{2} \mathrm{O}+\mathrm{O}_{2}
\end{aligned}
$$

Figure 3.1. The major ROS components produced by the phagocyte-derived oxidative burst

Upon stimulation, phagocytes produce large amounts of $\mathrm{O}_{2}{ }^{--}$which is quickly converted by SOD to oxygen and $\mathrm{H}_{2} \mathrm{O}_{2}$ (Reaction (i)). The $\mathrm{H}_{2} \mathrm{O}_{2}$ that is thereby generated is rapidly broken down into water and oxygen by the $P$. aeruginosa catalase, KatA (Reaction (ii)). 
As noted previously, the superoxide radical is unlikely to be the source of direct bacterial damage due to its inability to cross lipid membranes. Rather, it is believed that $\mathrm{H}_{2} \mathrm{O}_{2}$ is the toxic species most likely to require control by phagocyte-engulfed bacteria. By acting as both the hydrogen acceptor and donor, catalase enzymes rapidly detoxify otherwise lethal concentrations of $\mathrm{H}_{2} \mathrm{O}_{2}$. Catalase enzymes also are attractive defence mechanisms in terms of their efficiency and low energy expenditure, enabling catalytic degradation of $\mathrm{H}_{2} \mathrm{O}_{2}$ at times of stress without redirecting energy from alternative defence systems (80). Additionally, catalases can have a very high turnover rate (162) and are therefore not easily saturated, allowing for $\mathrm{H}_{2} \mathrm{O}_{2}$ control over a wide range of intracellular concentrations (114).

While the $P$. aeruginosa catalase KatA has a very high $k_{\text {cat }}$ for $\mathrm{H}_{2} \mathrm{O}_{2}$, it also has a very high $K_{m}$ (the substrate concentration at which half maximal velocity is achieved, and therefore an approximate measure of an enzyme's affinity for that substrate; for KatA the $K_{m}$ for $\mathrm{H}_{2} \mathrm{O}_{2}$ has been measured at $\left.44.7 \mathrm{mM}(110)\right)$. Although KatA can function at low concentrations of $\mathrm{H}_{2} \mathrm{O}_{2}$, this requires an additional hydrogen donor and the enzyme drops several orders of magnitude in catalytic efficiency $(114,163)$. Moreover, low concentrations of $\mathrm{H}_{2} \mathrm{O}_{2}(<0.6 \mathrm{mM})$ have actually been shown to be more dangerous in terms of forming oxidative DNA lesions (164). Therefore, it is possible that antioxidants with greater affinity for $\mathrm{H}_{2} \mathrm{O}_{2}$ may also contribute to guarding against oxidative stress. However, other $P$. aeruginosa catalases do not appear to fulfil this role (111). At low concentrations KatB is also upregulated by exposure to $\mathrm{H}_{2} \mathrm{O}_{2}$ but unlike KatA, does not demonstrate protective catalase activity (113). Studies by Elkins et al (1999; (113))and Ma et al (1999; (110)), also established that in the absence of KatA not only is KatB not catalytically active, but that $P$. aeruginosa still shows a high degree of tolerance to $\mathrm{H}_{2} \mathrm{O}_{2}$ assault, and therefore it was concluded that catalase activity alone is not the sole determinant of $\mathrm{H}_{2} \mathrm{O}_{2}$ resistance. More recently, water-soluble NQORs have been shown to confer $\mathrm{H}_{2} \mathrm{O}_{2}$ tolerance via an indirect mechanism of detoxification. 


\subsubsection{Soluble nitro- and quinone oxidoreductases}

Soluble NQORs are thought to play important roles in direct defence against quinone cytotoxicity; whereby redox cycling of quinone compounds can result in the production of high levels of $\mathrm{H}_{2} \mathrm{O}_{2}(165,166)$. Quinones are widely distributed compounds that share a core structure of a central six-carbon aromatic ring with two ketone substituents, and are best understood for their role in respiration as membrane bound electron acceptors in the electron transport chain. These compounds can exist in three distinct forms depending on their degree of oxidation-reduction: fully-oxidised (quinone); one-electron reduced (semi-quinone); two-electron reduced (quinol) (Figure 1.2). The semi-quinone form can react with molecular oxygen to produce the $\mathrm{O}_{2}{ }^{\bullet-}$ radical which, as described above (Figure 3.1), is a primary source of biological $\mathrm{H}_{2} \mathrm{O}_{2}$. This superoxide radical also reduces iron which, in the presence of $\mathrm{H}_{2} \mathrm{O}_{2}$ can catalyse formation of the particularly damaging hydroxyl radical $\left({ }^{\circ} \mathrm{OH}\right)$ via Fenton chemistry (167).

NQORs catalyse the reduction of quinones by accepting electrons from either NADH or NADPH thereby either (i) reducing the quinone by one electron to form the reactive semi-quinone, or (ii) simultaneously transferring two electrons to the quinone substrate to directly form quinol. These enzymes are typically flavoproteins, defined by possession of one or more bound flavin adenine dinucleotide (FAD) or flavin mononucleotide (FMN) cofactor per enzyme complex. NQORs can exist as either lipid membrane bound or soluble cytoplasmic flavoproteins $(46,47)$. It is the latter that are generally implicated in antioxidant defence. NQORs that employ a simultaneous two electron transfer mechanism for quinone reduction not only avoid formation of the highly reactive semi-quinone intermediate, but may also play a key role in maintaining a pool of reduced quinones that are in turn able to quench $\mathrm{H}_{2} \mathrm{O}_{2}$ directly $(115,117)$.

Although several soluble NQORs have now been identified based on structural and/or sequence conservation with mammalian DT-diaphorase, a 2005 study by Gonzalez et al was the first to demonstrate an overt role for NQORs in guarding bacteria against oxidative stress when challenged with $\mathrm{H}_{2} \mathrm{O}_{2}$ (Figure 3.2; (130)). The soluble chromate reductase (ChrR) from Pseudomonas putida was demonstrated to be important for $\mathrm{H}_{2} \mathrm{O}_{2}$ tolerance. Over-expression of $c h r R$ was able to quench exogenously added $3 \mathrm{mM} \mathrm{H}_{2} \mathrm{O}_{2}$ and reinitiate growth in $6 \mathrm{~h}$, whereas the $\Delta c h r R$ mutant took nearly 
$12 \mathrm{~h}$ to commence growth recovery in the presence of $\mathrm{H}_{2} \mathrm{O}_{2}$, a time point that corresponded with the extracellular $\mathrm{H}_{2} \mathrm{O}_{2}$ level dropping below $5 \mu \mathrm{M}$. Based on this previous work in a related Pseudomonas species, we hypothesized that $P$. aeruginosa would similarly utilise NQORs to withstand oxidative stress, particularly at lower levels where KatA is not so efficient.

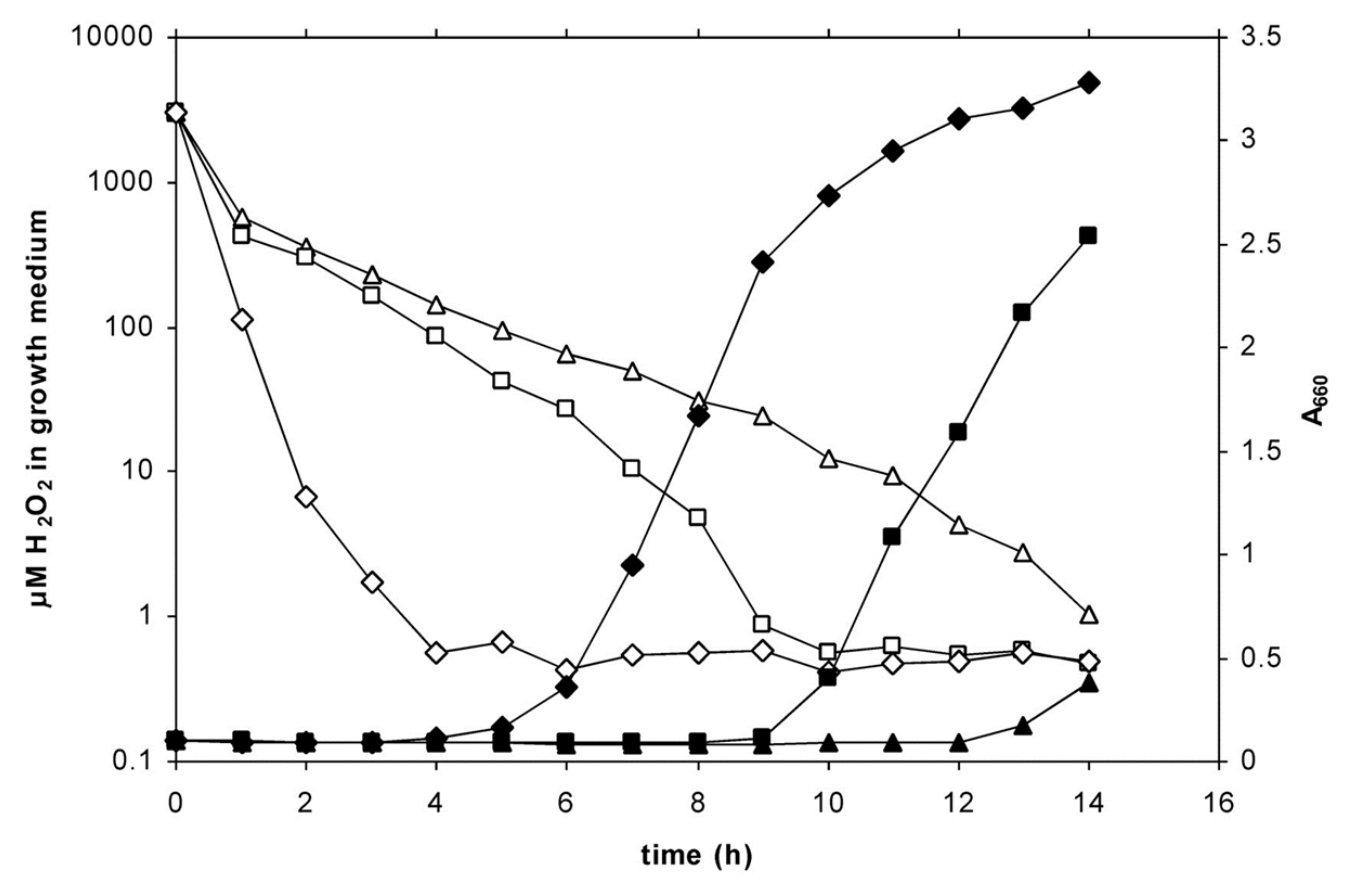

Figure 3.2. $\mathrm{H}_{2} \mathrm{O}_{2}$-scavenging compared to growth in $P$. putida

Strains of $P$. putida wild type $(\mathbf{\square}), \operatorname{chr} R$ mutant $(\boldsymbol{\Delta})$, and $c h r R+$ overexpression $(\downarrow)$ were inoculated to an initial $\mathrm{OD}_{660} 0.1$ in $\mathrm{LB}$ amended with $3 \mathrm{mM} \mathrm{H}_{2} \mathrm{O}_{2}$. Growth (closed symbols) was monitored at $\mathrm{OD}_{660}$ while residual $\mathrm{H}_{2} \mathrm{O}_{2}$ (open symbols) was assayed with the fluorescent dye Amplex ${ }^{\circledR}$ Red via excitation/emission at $540 \mathrm{~nm}$ and $585 \mathrm{~nm}$ respectively. This experiment was repeated in triplicate, with consistent results. The figure presents representative data from one of the replicates. Reproduced with permission from $\mathrm{Dr}$ David Ackerley (130).

Similar to ChrR of P. putida, enzymes from other bacterial species have also been characterised as divalent quinone reductases with antioxidant potential. WrbA, $\mathrm{NfsB}$, and NfsA of $E$. coli have been shown to exhibit divalent reduction of quinone substrates $(132,168,169)$. Similarly, MdaB of Helicobacter pylori, an enzyme that shares high levels of amino acid identity with mammalian DT-diaphorase, has also been shown to contribute to oxidative stress defence, both in vitro and in a mouse model $(128,170)$. Although NQORs are distributed widely throughout bacterial species, at the time this work began no roles for soluble NQOR-like enzymes in $P$. aeruginosa virulence had previously been described. 


\subsection{Objectives}

- Identify promising NQOR candidates of $P$. aeruginosa and characterise their ability to reduce quinone substrates as well as their mechanism of electron transfer (one electron vs. simultaneous two electron).

- Determine whether $P$. aeruginosa NQORs can confer protection against oxidative stress when the host cell is challenged with hydrogen peroxide in vitro.

\subsection{Results}

\subsubsection{Biochemical characterisation of $P$. aeruginosa $N A D(P) H$ quinone oxidoreductase enzymes (NQORs)}

\subsubsection{Selection of NQOR candidates for this study}

NQORs for this study were chosen based on (i) shared identity with P. putida ChrR; (ii) shared identity with mammalian DT-diaphorase; or (iii) shared identity with E. coli WrbA, MdaB, NfsA or NfsB, other bacterial quinone oxidoreductases that have been described as having a likely antioxidant role. In conducting BLAST searches of the $P$. aeruginosa genome, it became apparent that $P$. aeruginosa possesses several NQORs with shared protein identity to most of the above described enzymes from other species (although no NfsA-like enzyme was identified; Table 3.1). Using an arbitrarily selected cut off of $>25 \%$ protein identity with their closest NQOR orthologue, the following $P$. aeruginosa genes were selected for a targeted investigation: PA0949, PA1204, PA2580, PA4975, and PA5190 (Table 3.2). 
Table 3.1. Summary of NQORs found in P. aeruginosa

\begin{tabular}{|c|c|c|c|}
\hline Family $^{a}$ & Organism $^{b}$ & Locus ID ${ }^{c}$ & \%ID $^{d}$ \\
\hline NfsA & Escherichia coli & - & - \\
\hline \multirow[t]{2}{*}{ NfsB } & Escherichia coli & 3208 & $28 \%$ \\
\hline & & 5190 & $25 \%$ \\
\hline \multirow[t]{3}{*}{ MdaB } & Escherichia coli & 2580 & $64 \%$ \\
\hline & & 4975 & $26 \%$ \\
\hline & & 1224 & $29 \%$ \\
\hline WrbA & Escherichia coli & 0949 & $39 \%$ \\
\hline \multirow[t]{2}{*}{ ChrR } & Pseudomonas putida & 1204 & $46 \%$ \\
\hline & & 3223 & $31 \%$ \\
\hline \multirow{4}{*}{$\begin{array}{c}\text { DT- } \\
\text { Diaphorase }\end{array}$} & Homo sapiens & 4975 & $35 \%$ \\
\hline & & 1224 & $34 \%$ \\
\hline & & 1225 & $31 \%$ \\
\hline & & 0853 & $28 \%$ \\
\hline
\end{tabular}

${ }^{a}$ Family names were determined based on protein homology grouping with previously identified NQOR enzymes.

${ }^{\mathrm{b}}$ Organisms listed refer to the species origin of the previously identified NQOR orthologue.

${ }^{c}$ Locus ID describes the position of each P. aeruginosa gene within the annotated genome database (www.pseudmonas.com).

\%ID indicates the amino acid identity as calculated by the ClustalW program of each NQOR to the originally characterised species sequence.

Table 3.2. Targeted NQOR selection for this study

\begin{tabular}{lll}
\hline Gene & NQOR Family & Reference \\
\hline PA0949 & WrbA & $(132,171-173)$ \\
PA1204 & ChrR & This study \\
PA2580 & MdaB & $(129)$ \\
PA4975 & DT-diaphorase & This study \\
PA5190 & NfsB & This study \\
\hline
\end{tabular}

\subsubsection{In vitro quinone reduction by $P$. aeruginosa NQOR-like enzymes}

To investigate the ability of the $P$. aeruginosa NQORs to reduce quinones, as well as their mechanism of reduction (one electron vs. simultaneous two electron), purified Nterminal $\mathrm{His}_{6}$ tagged recombinant proteins were expressed from each target gene and purified by nickel chromatography as described in section 2.8. SDS-PAGE was used to confirm that the mass of the expressed enzyme was consistent with the predicted mass and also to examine the relative level of purification of soluble protein prior to commencing biochemical analysis (Figure 3.3). Each enzyme appeared yellow during and post purification, indicating that the flavin cofactors were co-purifying; however 
previous work in the Ackerley laboratory has demonstrated that there is always partial loss of cofactor during nickel affinity chromatography (Gareth Prosser, personal communication). Therefore each purified enzyme was incubated with FMN cofactor overnight (FAD for MdaB) prior to desalting and buffer exchange.

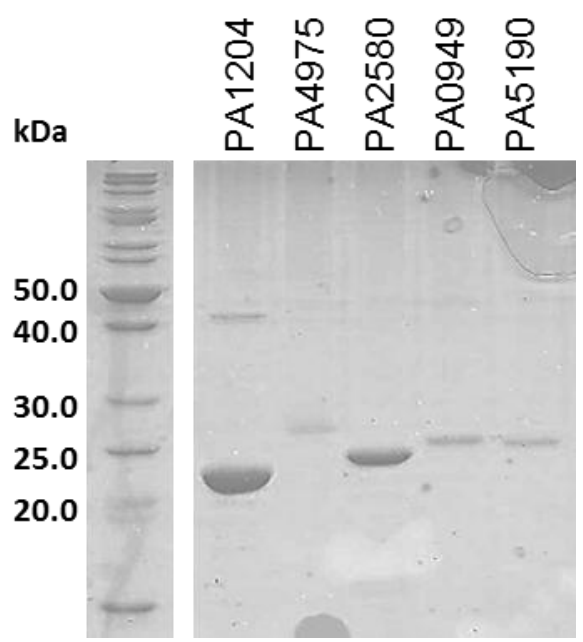

Figure 3.3. Purified His6 tagged NQORs on SDS-PAGE

Sequence-verified NQOR genes were over-expressed from pET28a(+) with as described in Section 2.8, purified by nickel affinity chromotography and run on SDS-PAGE. Each lane was loaded with $\sim 1 \mu \mathrm{g}$ of total protein as determined by the Bio-Rad $\mathrm{Dc}^{\mathrm{TM}}$ protein assay.

Each purified NQOR was tested for activity with three different quinone substrates, exhibiting varying degrees of water solubility: benzoquinone (BQ) - most soluble $(\log P=0.265) ;$ menadione $(M e n)$ - intermediate solubility $(\log P=1.6)$; coenzyme $Q_{1}(\mathrm{CoQ} 1)$ - least soluble $(\log P=2.2$; Figure 3.4$)$. Where activity was detected in preliminary assays, detailed measurements of the steady state (MichaelisMenten) kinetics were conducted. All five enzymes were quite active with $B Q$, but varied widely in their ability to metabolise the more lipophilic quinone substrates (Table 3.3). The most active enzymes, PA4975 and PA1204, had specificity constants for benzoquinone that were comparable to previously reported values for the $\mathrm{H}_{2} \mathrm{O}_{2}$ quenching ChrR of $P$. putida $\left(k_{\text {cat }} / K_{m}=8.5 \times 10^{6} \mathrm{M}^{-1} \mathrm{~s}^{-1} ;(130)\right)$. The remaining NQORs, PA2580, PA5190, and PA0949, were also able to reduce benzoquinone but at substantially reduced rates compared to PA4975 and PA1204. However, PA2580 was able to turn over the least soluble substrate, CoQ1, with a $k_{\text {cat }} / K_{m} 25$-fold higher than PA1204, the only other NQOR that exhibited CoQ1 reduction. 
<smiles>O=C1C=CC(=O)C=C1</smiles>

Benzoquinone<smiles>CC1=CC(=O)c2ccccc2C1=O</smiles><smiles>COC1=C(OC)C(=O)C(CC=C(C)C)=C(C)C1=O</smiles>

Coenzyme Q1

Figure 3.4. Structures of quinone substrates used to measure steady-state kinetic parameters

Three different quinone substrates were used in this study, each exhibiting different degrees of water solubility: benzoquinone (BQ) - most soluble menadione (Men) - intermediate solubility; coenzyme Q1 (CoQ1) - least soluble.

Table 3.3. Steady-state Michaelis-Menten kinetic parameters

\begin{tabular}{|c|c|c|c|c|c|}
\hline Protein & Substrate & $V_{\max }(\mu \mathrm{mol} / \mathrm{min} / \mathrm{mg})$ & $K_{m}(\mu M)^{b}$ & $k_{\text {cat }}\left(\mathrm{s}^{-1}\right)^{\mathrm{b}}$ & $k_{\text {cat }} / K_{m}\left(M^{-1} s^{-1}\right)$ \\
\hline \multirow{3}{*}{ PA4975 } & $B Q$ & $608 \pm 75$ & $321 \pm 52$ & $405 \pm 50$ & $1.3 \times 10^{6}$ \\
\hline & Men & $0.03 \pm 0.01$ & $1371 \pm 481$ & $0.02 \pm 0.01$ & $1.6 \times 10^{1}$ \\
\hline & CoQ1 & N.D. ${ }^{a}$ & N.D. ${ }^{a}$ & N.D. ${ }^{a}$ & N.D. ${ }^{a}$ \\
\hline \multirow{3}{*}{ PA1204 } & $B Q$ & $877 \pm 63$ & $288 \pm 35$ & $585 \pm 42$ & $2.0 \times 10^{6}$ \\
\hline & Men & $190 \pm 19$ & $775 \pm 93$ & $127 \pm 13$ & $1.6 \times 10^{5}$ \\
\hline & CoQ1 & $15 \pm 5.4$ & $1495 \pm 603$ & $10 \pm 3.5$ & $6.8 \times 10^{3}$ \\
\hline \multirow{3}{*}{ PA2580 } & $\mathrm{BQ}$ & $0.1 \pm 0.04$ & $0.3 \pm 0.28$ & $0.1 \pm 0.03$ & $3.0 \times 10^{5}$ \\
\hline & Men & $0.1 \pm 0.02$ & $0.4 \pm 0.27$ & $0.1 \pm 0.01$ & $2.2 \times 10^{5}$ \\
\hline & CoQ1 & $0.4 \pm 0.29$ & $2.6 \pm 2.3$ & $0.2 \pm 0.2$ & $1.4 \times 10^{5}$ \\
\hline \multirow{3}{*}{ PA5190 } & $\mathrm{BQ}$ & $0.03 \pm 0.01$ & $0.9 \pm 0.4$ & $0.02 \pm 0.01$ & $2.3 \times 10^{4}$ \\
\hline & Men & $0.03 \pm 0.01$ & $2.6 \pm 0.8$ & $0.02 \pm 0.01$ & $6.8 \times 10^{3}$ \\
\hline & CoQ1 & N.D. ${ }^{a}$ & N.D. ${ }^{a}$ & N.D. ${ }^{a}$ & N.D. ${ }^{a}$ \\
\hline \multirow{3}{*}{ PA0949 } & $\mathrm{BQ}$ & $0.1 \pm 0.03$ & $1.5 \pm 0.8$ & $0.04 \pm 0.02$ & $3.4 \times 10^{4}$ \\
\hline & Men & N.D. ${ }^{a}$ & N.D. ${ }^{a}$ & N.D. ${ }^{a}$ & N.D. ${ }^{a}$ \\
\hline & CoQ1 & N.D. ${ }^{a}$ & N.D. ${ }^{a}$ & N.D. ${ }^{a}$ & N.D. ${ }^{a}$ \\
\hline
\end{tabular}

\footnotetext{
${ }^{a}$ Not detectable
}

${ }^{\mathrm{b}}$ These are apparent values as measured at $250 \mu \mathrm{M}$ NADH 


\subsubsection{Simultaneous two electron transfer by $P$. aeruginosa NQORs}

With all NQOR candidates selected for this study having been shown to actively reduce quinone substrates, the mode of electron transfer was determined by implementation of a cytochrome $c$ trap. Previous work has indicated that cytochrome $c$ is rapidly reduced by benzo-semi-quinone, but not by benzoquinol; and that this reduction leads to a quantifiable increase in absorbance at $\mathrm{OD}_{550} \mathrm{~nm}$ that can be monitored spectrophotometrically (130). Therefore, appearance of the reduced cytochrome $c$ species in the presence of an NQOR-catalysed reaction indicates a preference for univalent electron transfer, while lack of reduced cytochrome $c$ signifies exclusive twoelectron transfers. Lipoyl dehydrogenase (LpDH), an obligate one-electron reducer from Clostridium kluyveri (purchased pre-purified from Sigma-Aldrich) was used as a positive control for cytochrome $c$ reduction (Figure 3.5). In this experiment, all five target NQORs demonstrated a preference for simultaneous two electron transfer. 


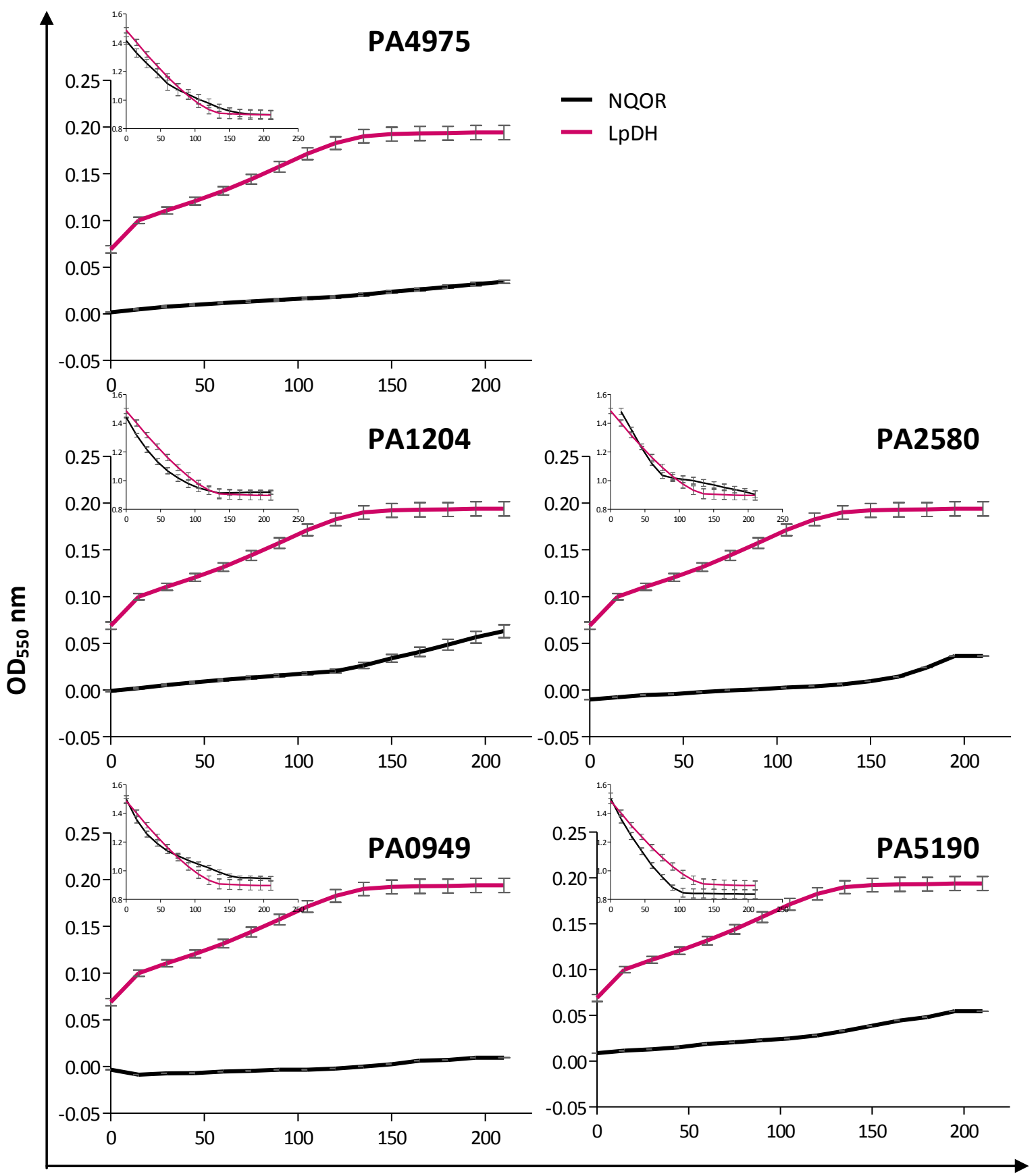

Time (s)

Figure 3.5. Determination of simultaneous two electron transfer - cytochrome $c$ trap Recombinant purified NQOR proteins (black) or control LpDH (red) at $0.1-5.0 \mu \mathrm{g} / \mathrm{mL}$ were mixed with $75 \mu \mathrm{M}$ cytochrome $c$ in the presence of $100 \mu \mathrm{M}$ benzoquinone substrate. Reactions were instigated by the addition of $250 \mu \mathrm{M} \mathrm{NADH}$ and were monitored over $5 \mathrm{~min}$ at $\mathrm{OD}_{550}$ for reduction of cytochrome $c$ (increase in absorbance). Inset, in each graph is the same reaction monitored at $\mathrm{OD}_{340}$ to observe the NADH consumption rate ( $y$-axis shows $O D_{340}$ and $x$-axis shows time in seconds). Each graph presents the mean of three replicates \pm 1 standard deviation. 


\subsubsection{Assessment of $\mathrm{NQOR} \mathrm{H}_{2} \mathrm{O}_{2}$-protective phenotypes in vitro}

With all NQOR candidates having demonstrated a primarily simultaneous two electron mechanism of BQ, these were further assessed for their ability to actively protect $P$. aeruginosa from $\mathrm{H}_{2} \mathrm{O}_{2}$ challenge in vitro. Preliminary trials to establish appropriate test concentrations for $P$. aeruginosa growth in media amended with $\mathrm{H}_{2} \mathrm{O}_{2}$ demonstrated that PAO1 is substantially less sensitive to $\mathrm{H}_{2} \mathrm{O}_{2}$ challenge than the $P$. putida strain (KT2440) that ChrR studies were conducted in. PAO1 exhibited substantial tolerance to $\mathrm{H}_{2} \mathrm{O}_{2}$ until concentrations exceeded $100 \mathrm{mM}$ (e.g. Figure 3.6A), whereas $P$. putida was unable to sustain growth in concentrations $>3 \mathrm{mM}$ (this study and (130); e.g. Figure 3.6B). At the very high concentrations of $\mathrm{H}_{2} \mathrm{O}_{2}$ required to impair growth of wild type PAO1, no protective effect conferred by NQOR gene over-expression was discernible (not shown). This in turn led us to consider whether or not the activity of the unusually active $P$. aeruginosa catalase, KatA, might potentially be interfering with any prospective NQOR phenotype in the presence of $\mathrm{H}_{2} \mathrm{O}_{2}$.

To test whether KatA might be "masking" NQOR contributions to $\mathrm{H}_{2} \mathrm{O}_{2}$ quenching, a $\triangle k a t A$ mutant in PAO1 was generated by in-frame gene deletion as described in section 2.9. When the $\Delta k a t A$ strain was challenged with $50 \mathrm{mM} \mathrm{H}_{2} \mathrm{O}_{2}$ no growth at all was evident across a $9 \mathrm{~h}$ time course, whereas a wild type PAO1 control grown in parallel recovered normally (Figure 3.6A). In contrast, when the $\Delta k a t A$ mutation was complemented by a katA gene over-expressed from plasmid $\mathrm{pSX}, \mathrm{H}_{2} \mathrm{O}_{2}$ tolerance and a wild type growth phenotype were restored (Figure 3.7A). Likewise, when the $P$. aeruginosa katA gene was over-expressed in $P$. putida KT2440, the ability of this bacterium to tolerate $6 \mathrm{mM} \mathrm{H}_{2} \mathrm{O}_{2}$ challenge was greatly enhanced, although it did not achieve the same levels of growth as the unchallenged control (Figure 3.7B). Nonetheless, these findings together provide evidence that $P$. aeruginosa PAO1 is inherently more resistant to $\mathrm{H}_{2} \mathrm{O}_{2}$ challenge than $P$. putida $\mathrm{KT} 2440$ due to its powerful KatA catalase. 
A

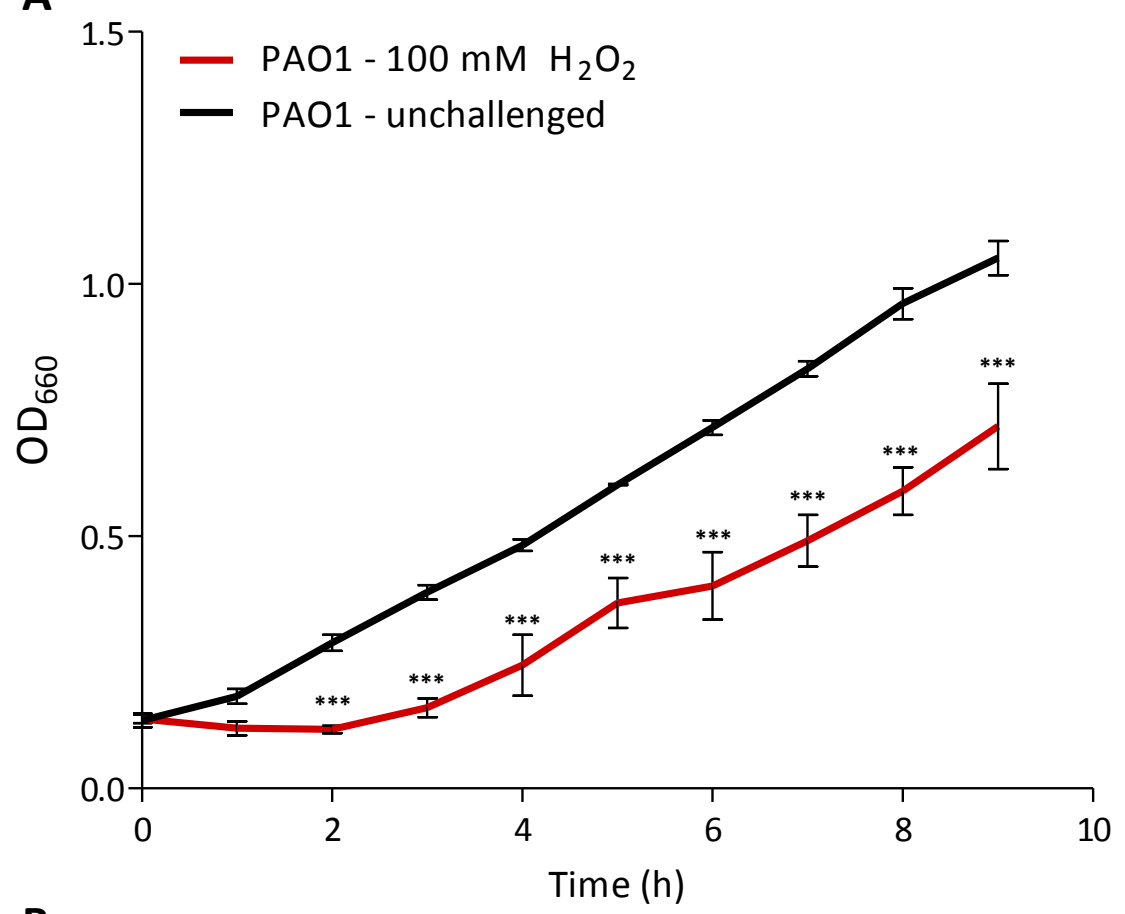

B

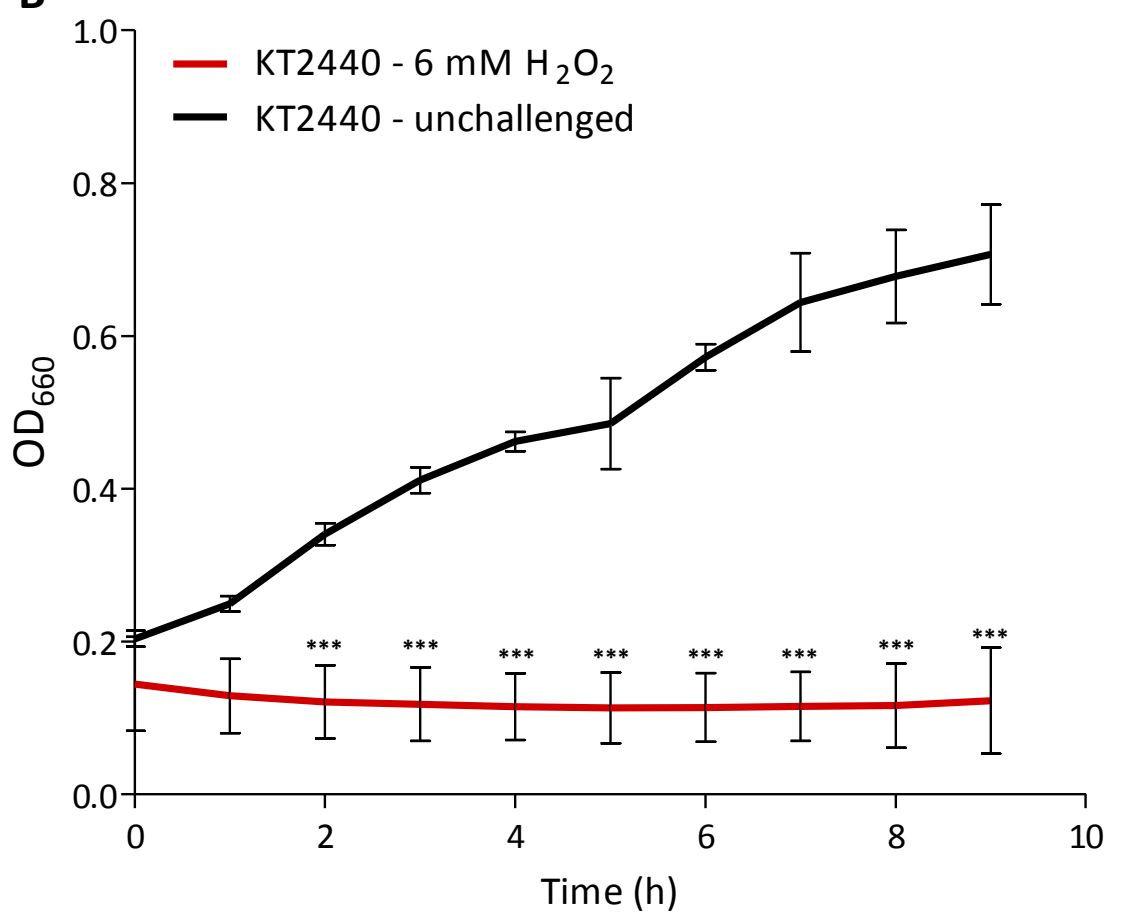

Figure 3.6. Growth of wild type $P$. aeruginosa $\mathrm{PAO} 1$ and $P$. putida $\mathrm{KT} 2440$ in $\mathrm{H}_{2} \mathrm{O}_{2}$ amended media

Wild type strains of (A) PAO1 or (B) KT2440 were inoculated to an initial $\mathrm{OD}_{660}$ of 0.1 in LB amended with $100 \mathrm{mM} \mathrm{H}_{2} \mathrm{O}_{2}$, or $6 \mathrm{mM} \mathrm{H}_{2} \mathrm{O}_{2}$ respectively, and growth was monitored at $\mathrm{OD}_{660}$ over $9 \mathrm{~h}$. The plotted $\mathrm{OD}_{660}$ data are means of 3 independent experiments, each performed in duplicate, and error bars are \pm 1 standard deviation. Significance is reported where $p<0.001(* * *)$ is compared to unchallenged controls. 

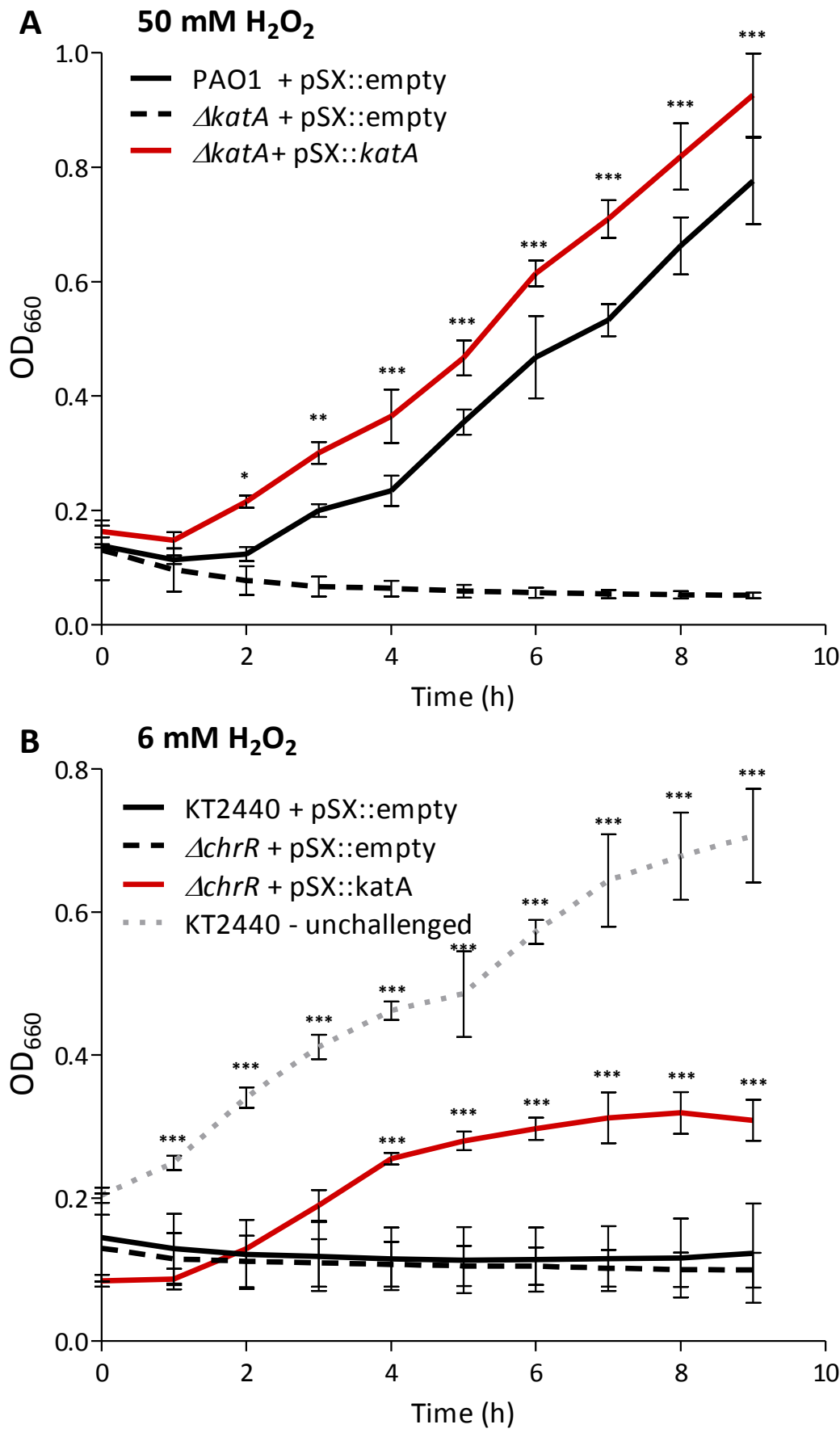

Figure 3.7. Effect of $k a t A$ over-expression in different $P$. aeruginosa PAO1 and $P$. putida KT2440 backgrounds

(A) Growth of wild type $P$. aeruginosa PAO1 bearing an empty control plasmid (PAO1 + pSX::empty) and the $\triangle$ katA mutant bearing either an empty control plasmid ( $\triangle k a t A+\mathrm{pSX}:: e m p t y)$ or pSX over-expressing the katA gene $(\Delta k a t A+\mathrm{pSX}:: k a t A)$ in the presence of $50 \mathrm{mM} \mathrm{H}_{2} \mathrm{O}_{2}$. (B) Growth of wild type $P$. putida KT2440 bearing an empty control plasmid (KT2440 + pSX::empty) and the $\Delta c h r R$ mutant bearing either an empty control plasmid ( $\triangle c h r R+\mathrm{pSX}:$ :empty) or pSX over-expressing the katA gene $(\Delta c h r R+$ pSX::katA) in the presence of $6 \mathrm{mM} \mathrm{H}_{2} \mathrm{O}_{2}$. Growth of an unchallenged wild type P. putida KT2440 control is also indicated. In both (A) and (B) the pSX plasmids were induced and maintained by amending the growth media with $0.25 \mathrm{mM}$ IPTG and $10 \mu \mathrm{g} / \mathrm{ml}$ gentamycin. Strains were inoculated to an initial $O_{660}$ of 0.1 in LB amended with $\mathrm{H}_{2} \mathrm{O}_{2}$ as indicated and growth was monitored by hourly $\mathrm{OD}_{660}$ readings over a $9 \mathrm{~h}$ timecourse. The plotted $\mathrm{OD}_{660}$ data are means of 3 independent experiments, each performed in duplicate, and error bars are \pm 1 standard deviation. Significance is reported where $p<0.05(*), p<0.01$ $\left({ }^{* *}\right)$, and $\mathrm{p}<0.001\left({ }^{* *}\right)$ is compared to $(\mathrm{A}) \Delta k a t A+\mathrm{pSX}:$ :empty, and $(\mathrm{B}) \Delta c h r R+\mathrm{pSX}:$ :empty controls. 


\subsubsection{NQOR Gene-deletion}

Having shown that KatA would be likely to "mask" any $\mathrm{H}_{2} \mathrm{O}_{2}$-sensitive phenotype resulting from NQOR gene-deletion, we aimed to investigate NQOR mutants in katAnull strains of PAO1. Creation of a gene knockout mutant strain for each of the NQORs listed in Table 3.2 was attempted, and an in-frame gene deletion of $\Delta 1204$ was successfully generated in both wild type PAO1 and the $\Delta k a t A$ mutant. Unfortunately, we were unable to replicate this success for any of the remaining four NQOR candidates, despite employing a large number of different mutagenesis strategies and culture conditions, including: use of four different suicide vectors bearing different antibiotic markers; increasing the regions of homology used to create recombination events; testing of a variety of selection media; and use of a range of historical and contemporary mating protocols, such as triparental and biparental filter mating, electroporation and heat shock transformation. Ultimately we were therefore only able to test the single mutant $\Delta 1204$ and double mutant $\Delta k a t A \Delta 1204$ strains of PAO1 for heightened sensitivity to challenge by $\mathrm{H}_{2} \mathrm{O}_{2}$.

Based on the ability of PA1204 to reduce all three quinone substrates tested in the purified protein assays, its apparent preference for simultaneous two electron transfer, and $46 \%$ amino acid identity with ChrR of P. putida KT2440 which has a proven antioxidant role, there was strong biochemical evidence to suspect PA1204 would, like ChrR, perform as an antioxidant enzyme in vitro. However, the single mutant, $\Delta 1204$, grew no differently from wild type PAO1 at all concentrations tested (0 $\mathrm{mM}$ to $275 \mathrm{mM}$; e.g. Figure 3.7). Moreover, the double mutant $\Delta k a t A \Delta 1204 \mathrm{did}$ not appear to be more sensitive to $\mathrm{H}_{2} \mathrm{O}_{2}$ than the $\Delta k a t A$ mutant (e.g. Figure 3.7.). At micromolar $\mathrm{H}_{2} \mathrm{O}_{2}$ concentrations as low as $0.4 \mathrm{mM}$, the $\Delta k a t A \Delta 1204$ mutant began to appear slightly more sensitive than $\Delta k a t A$, however, this difference was not statistically significant at any time point or concentration of $\mathrm{H}_{2} \mathrm{O}_{2}$ tested. 


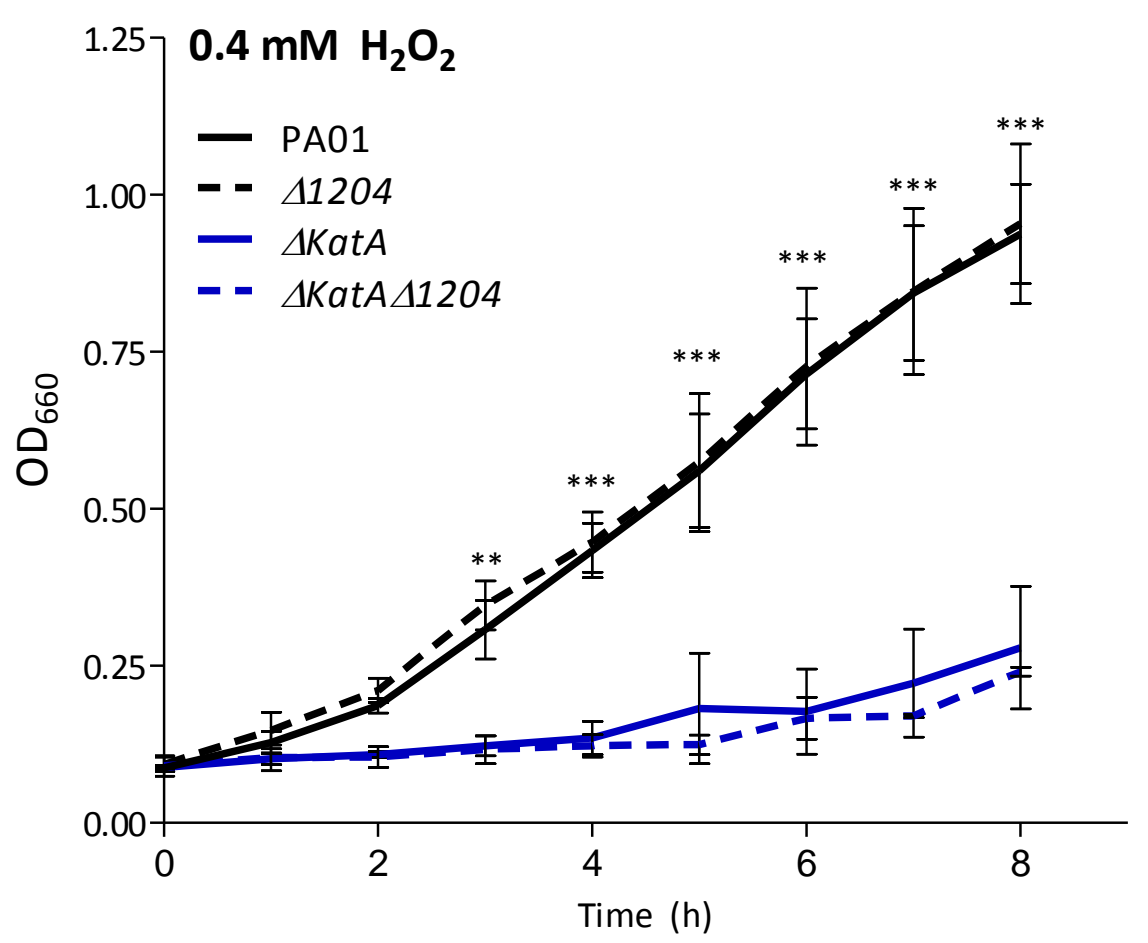

Figure 3.8. Growth by mutant PAO1 strains in $0.4 \mathrm{mM} \mathrm{H}_{2} \mathrm{O}_{2}$

The wild type $\mathrm{PAO} 1, \Delta 1204, \Delta k a t A$, and $\Delta k a t A \Delta 1204$ strains were inoculated to an $\mathrm{OD}_{660} 0.1$ in LB amended with $0.4 \mathrm{mM} \mathrm{H}_{2} \mathrm{O}_{2}$ and monitored at $\mathrm{OD}_{660}$ over $9 \mathrm{~h}$. These graphs indicate growth measured by $\mathrm{OD}_{660}$ and are the means of 3 independent experiments, each performed in duplicate, and error bars are \pm 1 standard deviation. Significance is reported where $p<0.01\left(^{* *}\right)$ and $p<0.001(* * *)$ are compared to $\triangle K a t A$.

\subsubsection{Effect of $P$. aeruginosa NQOR over-expression on $P$. aeruginosa $P A O 1$} and $P$. putida $\mathrm{KT} 2440$ survival in $\mathrm{H}_{2} \mathrm{O}_{2}$-amended media

We next sought to determine whether over-expression of the $P$. aeruginosa NQOR candidates in the $\triangle k a t A$ strain might reveal contributions to oxidative stress resistance that were not discernible in the wild type background. These NQOR candidates were also over-expressed in the $\Delta c h r R$ mutant of $P$. putida to determine whether they could restore tolerance to $\mathrm{H}_{2} \mathrm{O}_{2}$ challenge in a manner consistent with the activity of ChrR as depicted in Figure 3.2.

When the NQOR candidates PA0949, PA1204, PA4975 and PA5190 (omitting PA2580 for reasons that are discussed in section 3.3.2.3) were individually overexpressed from pSX in the $P$. aeruginosa $\Delta$ katA strain at a range of $\mathrm{H}_{2} \mathrm{O}_{2}$ concentrations (0 mM, 0.2 mM, $0.4 \mathrm{mM}, 0.8 \mathrm{mM}, 1.5 \mathrm{mM}, 3 \mathrm{mM}, 6 \mathrm{mM}, 12.5 \mathrm{mM}, 25 \mathrm{mM}, 50 \mathrm{mM}$, and $100 \mathrm{mM}$ ), it was found that in each case the over-expression strain was able to 
tolerate micromolar and low-millimolar concentrations of $\mathrm{H}_{2} \mathrm{O}_{2}$. Surprisingly however, the $\Delta$ katA strain containing an empty vector control now tolerated these $\mathrm{H}_{2} \mathrm{O}_{2}$ concentrations just as well. This was in stark contrast to the growth phenotype of the $\Delta k a t A$ strain depicted in Figure 3.7, with the only experimental difference being the presence of the pSX plasmid and the addition of $0.25 \mathrm{mM}$ IPTG and $10 \mu \mathrm{g} / \mathrm{mL}$ gentamycin to the growth medium to maintain that plasmid. Using PA4975 as an example, the $\triangle k a t A$ strain containing pSX::PA4975 reached an optical density similar to wild type PAO1 by $9 \mathrm{~h}$ post challenge with $0.8 \mathrm{mM} \mathrm{H}_{2} \mathrm{O}_{2}$, but so did the empty plasmid control $\Delta k a t A+p S X:: e m p t y$ (Figure 3.8A). The only instance where NQOR overexpression in the $\Delta k a t A$ strain appeared to reproducibly confer protection to $\mathrm{H}_{2} \mathrm{O}_{2}$ challenge relative to the empty plasmid control was for PA0949 at $1.5 \mathrm{mM}$ (Figure 3.8B). 

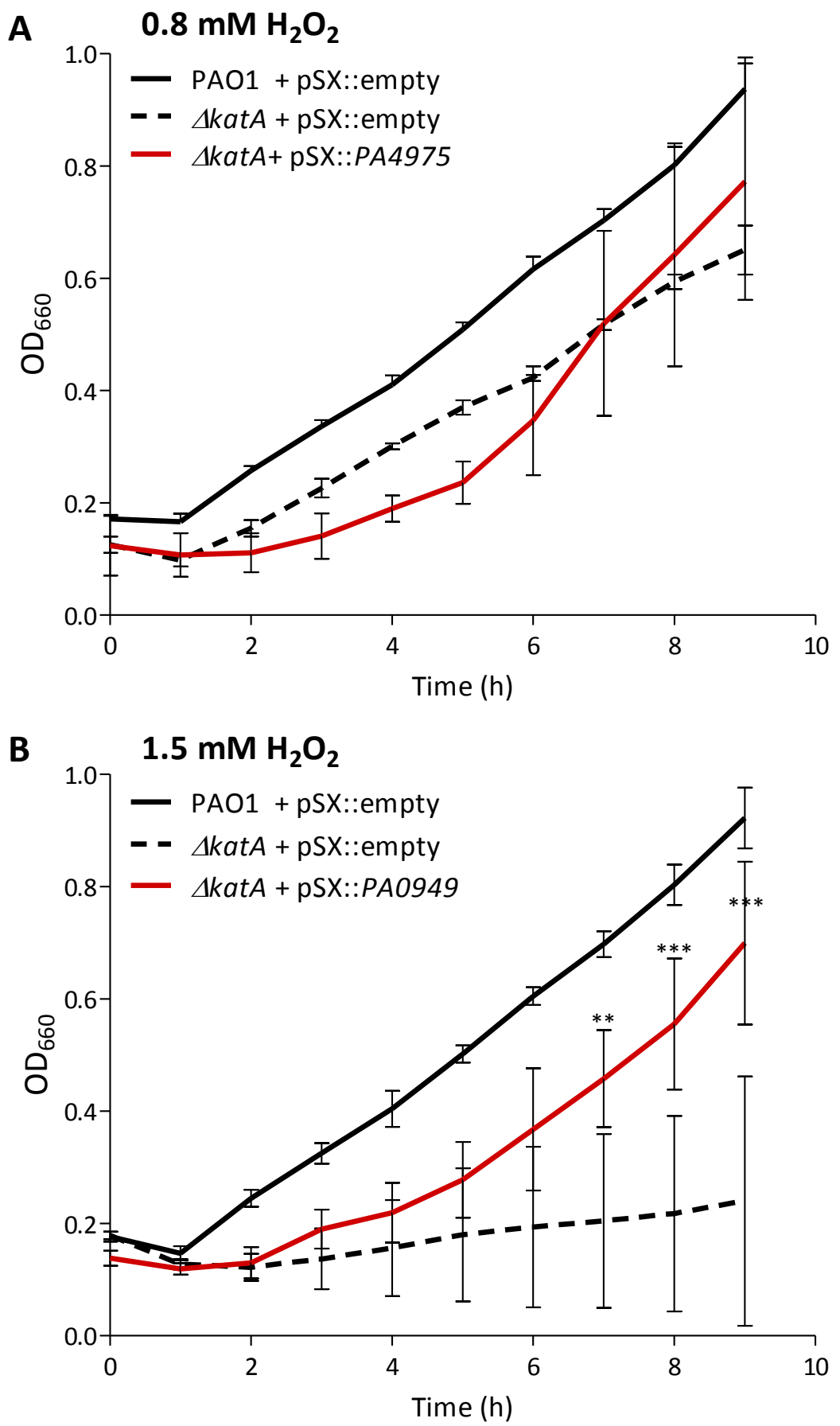

\section{Figure 3.9. PA4975 and PA0949 over-expression in PAO1}

(A) Growth of wild type $P$. aeruginosa PAO1 bearing an empty control plasmid (PAO1 + pSX::empty) and the $\triangle$ katA mutant bearing either an empty control plasmid ( $\triangle k a t A+p S X:: e m p t y)$ or pSX over-expressing the PA4975 gene ( $\triangle k a t A+p S X:: P A 4975)$ in the presence of $0.8 \mathrm{mM} \mathrm{H}_{2} \mathrm{O}_{2}$. (B) Growth of wild type $P$. aeruginosa PAO1 bearing an empty control plasmid (PAO1 + pSX::empty) and the $\triangle k a t A$ mutant bearing either an empty control plasmid ( $\triangle$ katA + pSX::empty) or pSX over-expressing the PA0949 gene ( $\triangle k a t A+$ pSX::PA0949) in the presence of $1.5 \mathrm{mM} \mathrm{H}_{2} \mathrm{O}_{2}$. In both (A) and (B) the pSX plasmids were induced and maintained by amending the growth media with $0.25 \mathrm{mM} I P T G$ and $10 \mu \mathrm{g} / \mathrm{mL}$ gentamycin. Strains were inoculated to an initial $\mathrm{OD}_{660}$ of 0.1 in $\mathrm{LB}$ amended with $\mathrm{H}_{2} \mathrm{O}_{2}$ as indicated and growth was monitored by hourly $\mathrm{OD}_{660}$ readings over a $9 \mathrm{~h}$ time course. The plotted $\mathrm{OD}_{660}$ data are means of 3 independent experiments, each performed in duplicate, and error bars are \pm 1 standard deviation. Significance is reported where $p<0.01\left({ }^{* *}\right)$ and $p<0.001\left({ }^{* * *}\right)$ are compared to $\Delta k a t A+p S X::$ empty. 
The apparent ability of the pSX::empty plasmid and concomitant IPTG and gentamycin to promote $\mathrm{H}_{2} \mathrm{O}_{2}$ tolerance in the $\Delta k a t A$ strain was proven by a head-tohead comparison of the plasmid free $\Delta k a t A$ strain grown in unamended LB vs. the $\Delta k a t A+p S X:: e m p t y$ strain grown in LB containing $0.25 \mathrm{mM}$ IPTG and $10 \mu \mathrm{g} / \mathrm{ml}$ gentamycin, across a wide range of $\mathrm{H}_{2} \mathrm{O}_{2}$ concentrations (Figure 3.9).

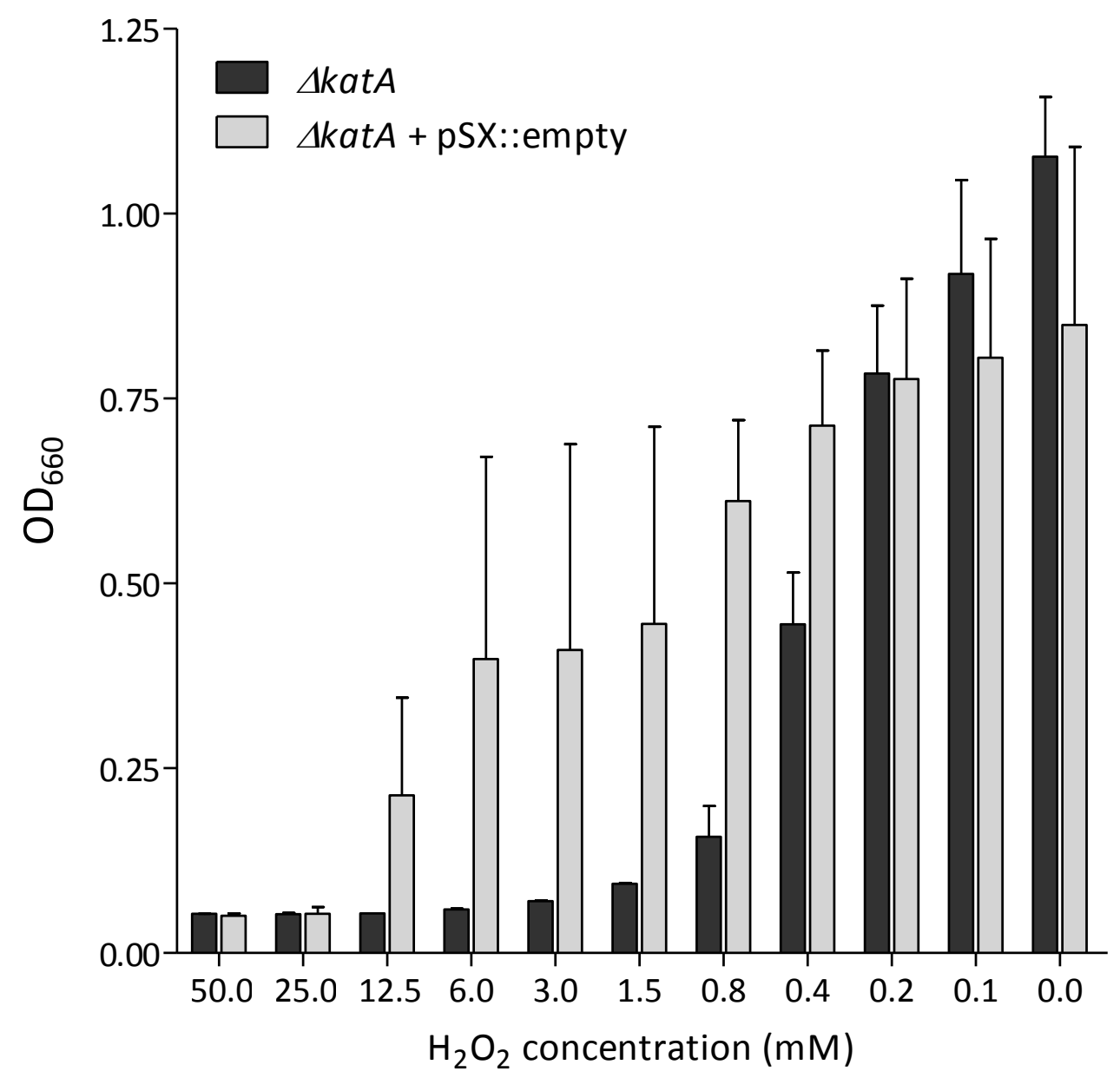

Figure 3.10. The influence of the pSX over-expression vector on $\mathrm{H}_{2} \mathrm{O}_{2}$ tolerance by the $\Delta$ katA strain

The $\triangle k a t A$ and $\triangle k a t A+p S X:: e m p t y$ strains were inoculated to an initial $\mathrm{OD}_{660}$ of 0.1 in $L B$ or $L B$ amended with $0.25 \mathrm{mM}$ IPTG and $10 \mu \mathrm{g} / \mathrm{mL}$ gentamycin, respectively, and 2-fold serial dilutions of $100 \mathrm{mM}$ down to $0.1 \mathrm{mM} \mathrm{H}_{2} \mathrm{O}_{2}$, as well as an unchallenged control for each strain. Cultures were monitored at $\mathrm{OD}_{660}$ over $9 \mathrm{~h}$. These graphs indicate end-point growth measured by $\mathrm{OD}_{660}$ at $9 \mathrm{~h}$ post treatment, and are the means of 9 independent experiments, each performed in duplicate, with error bars \pm 1 standard deviation.

Even more curiously, the induction of $\mathrm{H}_{2} \mathrm{O}_{2}$ tolerance conferred by the presence of pSX/IPTG/gentamycin appeared to be a $P$. aeruginosa specific phenomenon, which did not manifest in P. putida KT2440. Moreover, with the exception of PA1204 over- 
expression of all $P$. aeruginosa NQORs in the $\Delta c h r R$ mutant of $P$. putida was found to improve tolerance of $\mathrm{H}_{2} \mathrm{O}_{2}$. For example, $\mathrm{PA} 4975$ was reproducibly able to recover the P. putida $\Delta c h r R$ strain to near wild type levels of growth at $\leq 0.8 \mathrm{mM} \mathrm{H}_{2} \mathrm{O}_{2}$, whereas the $\triangle c h r R$ pSX::empty control strain remained sensitive (Figure 3.10). PA5190 had a fairly similar effect to PA4975, but PA2580 and PA0949 had an even more profound effect, conferring $\mathrm{H}_{2} \mathrm{O}_{2}$ tolerance to the $P$. putida $\Delta c h r R$ strain at concentrations as high as 6 $\mathrm{mM}$ (not shown).

The reason for a $P$. aeruginosa-specific growth advantage for the pSXcontaining strain in the presence of $\mathrm{H}_{2} \mathrm{O}_{2}$ is not understood. However, as the phenomenon is undoubtedly complex, and likely to be primarily of technical interest, it was not pursued further in this study.

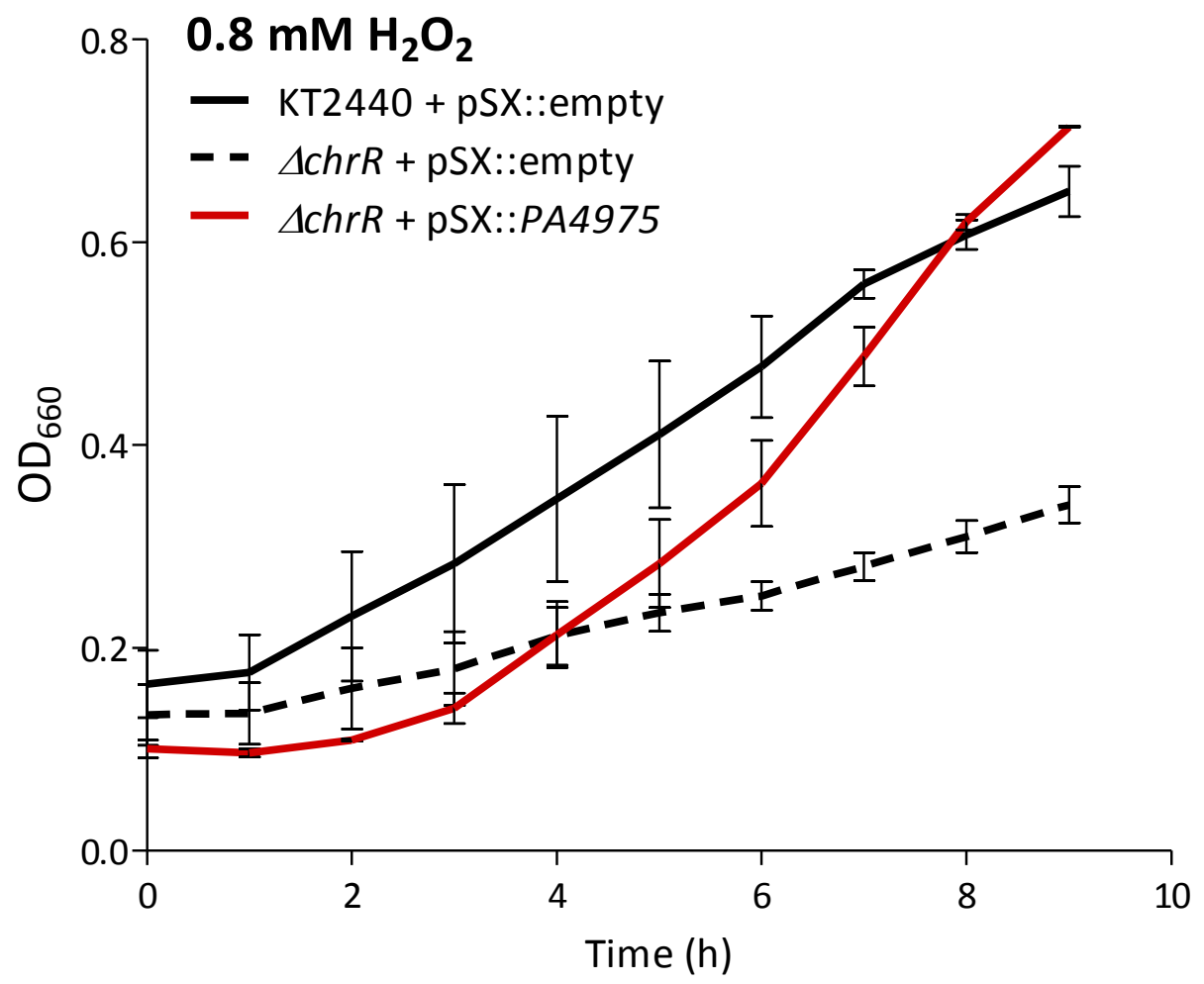

Figure 3.11. PA4975 over-expression in $\mathrm{KT} 2440$ at $0.8 \mathrm{mM} \mathrm{H}_{2} \mathrm{O}_{2}$

Growth of wild type P. putida KT2440 bearing an empty control plasmid (KT2440 + pSX::empty) and the $\triangle c h r R$ mutant bearing either an empty control plasmid ( $\triangle c h r R+p S X:: e m p t y)$ or pSX over-expressing the $P A 4975$ gene $(\triangle c h r R+p S X:: P A 4975)$ in the presence of $0.8 \mathrm{mM} \mathrm{H}_{2} \mathrm{O}_{2}$. The $\mathrm{pSX}$ plasmids were induced and maintained by amending the growth media with $0.25 \mathrm{mM}$ IPTG and $10 \mu \mathrm{g} / \mathrm{mL}$ gentamycin. Strains were inoculated to an initial $\mathrm{OD}_{660}$ of 0.1 in $\mathrm{LB}$ amended with $\mathrm{H}_{2} \mathrm{O}_{2}$ as indicated and growth was monitored by hourly $\mathrm{OD}_{660}$ readings over a $9 \mathrm{~h}$ time course. The plotted $\mathrm{OD}_{660}$ data are means of 3 independent experiments, each performed in duplicate, and error bars are \pm 1 standard deviation. Significance is reported where $p<0.05\left({ }^{*}\right), p<0.01(* *)$, and $p<0.001(* * *)$ is compared to $\Delta c h r R+$ pSX::empty. 


\subsubsection{Over-expression of $\mathrm{PA} 2580$ in P. aeruginosa $\mathrm{PAO1}$}

The PA2580 over-expression strain of $P$. aeruginosa was not discussed in section 3.3.2.2 because for an unknown reason over-expression of PA2580 (from pSX::PA2580) was found to inhibit growth of $P$. aeruginosa relative to either plasmid-free $P$. aeruginosa, or cells containing the empty plasmid (pSX::empty), independent of whether $\mathrm{H}_{2} \mathrm{O}_{2}$ challenge was applied (Figure 3.11A). Thus, growth of this strain could not be directly compared to growth of the empty plasmid control in any meaningful way. However, it was shown that if PA2580 growth data in response to $\mathrm{H}_{2} \mathrm{O}_{2}$ challenge is presented as a ratio of growth compared to its own unchallenged control (as per Figure 3.11C), PA2580 appears to confer substantial tolerance to $\leq 6 \mathrm{mM} \mathrm{H}_{2} \mathrm{O}_{2}$ whereas the $\Delta k a t A+p S X:: e m p t y$ strain remains sensitive (e.g. Figure 3.11C). 
A

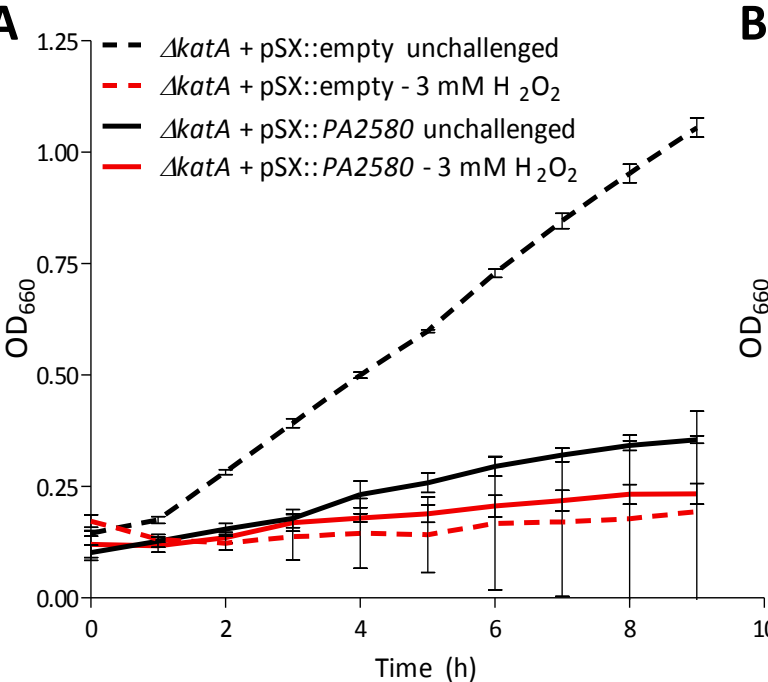

C

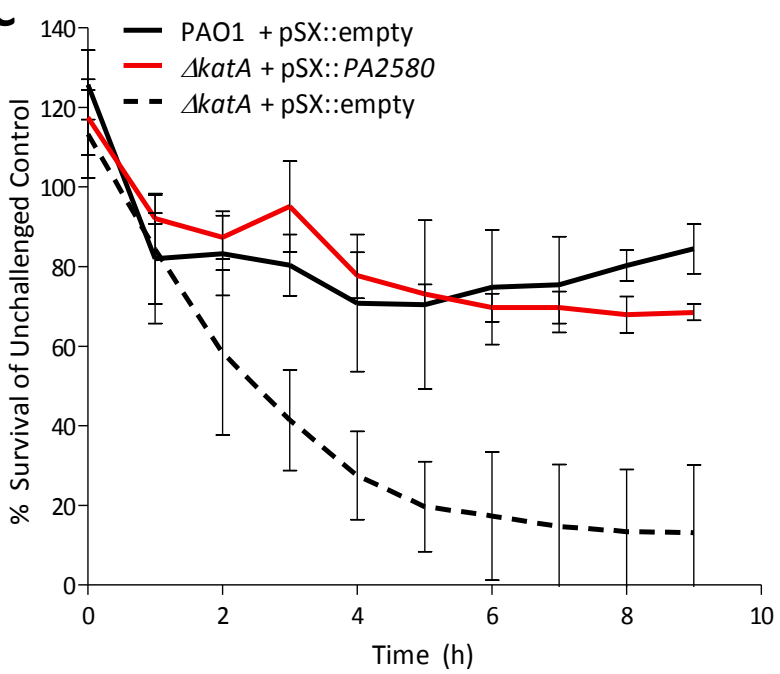

B

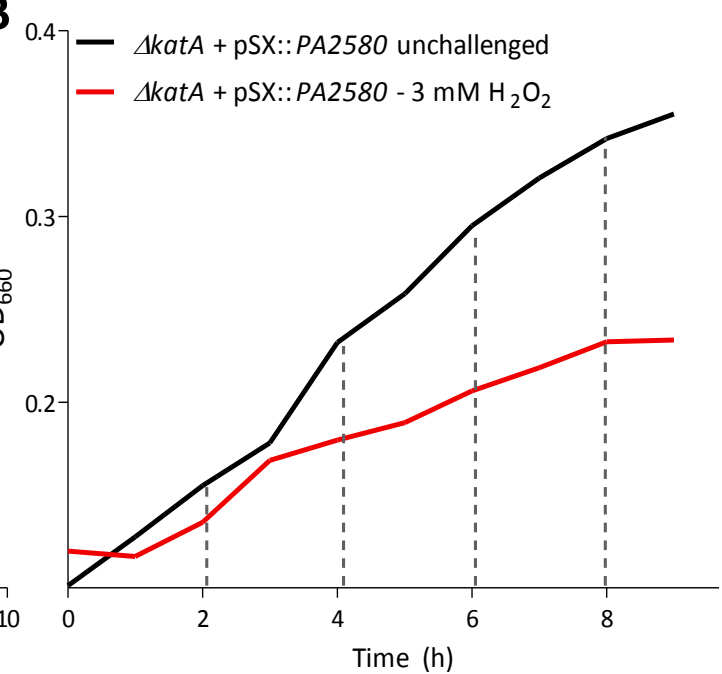

Figure 3.12. Conversion of $\mathrm{OD}_{660}$ to percentage growth for PA2580 over-expressing $P$. aeruginosa $\mathrm{PAO1}$ relative to the empty-plasmid control

(A) $\triangle k a t A+p S X:: e m p t y$ and $\triangle k a t A+p S X:: P A 2580$ were inoculated to an initial $O_{660}$ of 0.1 in LB amended with $0.25 \mathrm{mM} I P T G, 10 \mu \mathrm{g} / \mathrm{mL}$ gentamycin and $\pm 3 \mathrm{mM} \mathrm{H}_{2} \mathrm{O}_{2}$. The $\mathrm{OD}_{660}$ of challenged and unchallenged cultures was measured hourly for $9 \mathrm{~h}$. The plotted $\mathrm{OD}_{660}$ data are means of 3 independent experiments, each performed in duplicate, and error bars are \pm 1 standard deviation. (B) The $\mathrm{OD}_{660}$ data for the $\triangle k a t A+p S X:: P A 2580$ strain from graph $(\mathrm{A})$ is plotted on an $\mathrm{OD}_{660}$ scale 0.0 to 0.4 and the ratio of growth between challenged and unchallenged strains is calculated as the difference in OD at each time point measured. (C) The ratios of $\mathrm{OD}_{660}$ for unchallenged to challenged strains for each of $\triangle k a t A+$ pSX::empty and $\triangle k a t A+p S X:: P A 2580$ are presented as a percentage of survival at each time point measured. 


\subsection{Discussion}

A wealth of genome sequence data released over the last few years has revealed that soluble NQOR enzymes are ubiquitous in bacteria. Despite this, there is a dearth of information regarding their primary biological role(s). A few biochemical studies have focused on the simultaneous two electron quinone metabolising mechanisms of individual enzymes to speculate about a possible role in oxidative stress protection; these include WrbA from E. coli (132); ChrR from P. putida (130); and MdaB from $H$. pylori $(128,170)$ and M. tuberculosis (Rv3303c; (131)). However, only ChrR in P. putida and $\mathrm{MdaB}$ in $\mathrm{H}$. pylori have ever actually been found to exhibit an antioxidant phenotype. Here we demonstrate that protein identity, soluble quinone metabolism, and simultaneous two electron transfer alone does not directly implicate soluble NQORs as $\mathrm{H}_{2} \mathrm{O}_{2}$-protecting virulence factors in $P$. aeruginosa; but that nonetheless a contribution toward $\mathrm{H}_{2} \mathrm{O}_{2}$ tolerance can be observed for certain enzymes in an appropriate genetic background. In $P$. aeruginosa $\mathrm{PAO} 1$ this required a deletion of the gene encoding the potent KatA catalase, which was otherwise found to "mask" any contribution to $\mathrm{H}_{2} \mathrm{O}_{2}$ tolerance provided by individual NQORs.

Although PA1204 had the highest specificity constant for benzoquinone, the most soluble quinone substrate, gene-deletion of PA1204 failed to produce an $\mathrm{H}_{2} \mathrm{O}_{2}$ sensitive phenotype in even the $\Delta k a t A$ background. Despite our best efforts, genedeletion in PAO1 was largely unsuccessful; and we believe this is more likely to be a technical issue rather than due to the essential nature of the target genes, as the genes encoding PA0949, PA2580 and PA4975 have all been successfully knocked out by transposon insertion in P. aeruginosa strain PA14 (174). PA14 is widely regarded as being substantially more genetically tractable than PAO1, and may have proved a much easier strain to work with (Dr Jane Hill, personal communication); however PAO1 is still the most widely used strain for study of $P$. aeruginosa physiology and pathogenesis, and primarily for that reason was selected for this research. .

Greater success was observed when candidate NQORs were tested by overexpression from a broad host range plasmid. In the $\Delta k a t A$ background we found that PA2580 (as measured by percent survival relative to the unchallenged isogenic control) and PA0949 conferred significant improvements in growth relative to the empty plasmid control at up to $6 \mathrm{mM}$ and at $1.5 \mathrm{mM} \mathrm{H}_{2} \mathrm{O}_{2}$ challenge respectively. The same 
two NQORs were also found to be the most effective at promoting $\mathrm{H}_{2} \mathrm{O}_{2}$ tolerance in the $P$. putida $\triangle$ chrR strain, but in this heterologous host PA4975 and PA5190 were also found to promote growth at sub-millimolar levels of $\mathrm{H}_{2} \mathrm{O}_{2}$ challenge. An important difference between the two scenarios is that the $P$. aeruginosa $\Delta k a t A$ strain still contained endogenous copies of all the NQOR genes, which may have lessened the effect of candidate NQOR over-expression; whereas $P$. putida KT2440 does not appear to contain the same level of redundancy of soluble NQOR genes. Another possible explanation for PA5190 and PA4975 being found to confer protection in P. putida and not $P$. aeruginosa is that there may be a different complement of quinone substrates available for reduction between the two organisms. Studying this hypothesis further would require access to metabolomics equipment and expertise.

Although PA2580 did appear to provide the most substantial improvement in $\mathrm{H}_{2} \mathrm{O}_{2}$ tolerance of any NQOR in the $P$. aeruginosa $\triangle$ katA strain, this interpretation was complicated by the substantial reduction in growth caused by over-expression of this NQOR. PA2580 was the most promiscuous of our NQOR candidates, exhibiting fairly consistent levels of activity (specificity constant ca. $1-3 \times 10^{5} \mathrm{M}^{-1} \mathrm{~s}^{-1}$ ) with each of the three quinone substrates tested in our in vitro kinetic assays, despite their quite different partition coefficients $(\log P)$. Although highly speculative, it may be that the retarded growth of the PA2580 over-expressing strain was a consequence of this ability to effectively reduce less soluble, and perhaps membrane associated, quinone substrates. As the membrane associated quinones play key roles in the electron transport chain and therefore cellular respiration (175), an undesirable perturbation of this homeostasis may have promoted a detrimental growth phenotype. Another $P$. aeruginosa specific growth anomaly came in the form of the apparent ability of the pSX plasmid and/or associated IPTG and gentamycin to promote $\mathrm{H}_{2} \mathrm{O}_{2}$ tolerance in the $P$. aeruginosa $\Delta k a t A$ strain. Although this phenomenon was also not pursued further it does serve to highlight the profound influence (and potential for artefactual results) that slight variations in growth conditions can have on phenotype; a theme that is expanded upon in Chapter 4 also.

Although NQORs are distributed widely throughout bacterial species, at the time this work began, no roles for soluble NQOR-like enzymes in $P$. aeruginosa virulence had previously been described. However, the large selection of NQOR-like genes in the PAO1 genome, and the likelihood that, with the genetic flexibility of $P$. 
aeruginosa, many of these enzymes may display an overlap in function, suggested that adopting a more global strategy to interrogate NQOR function might prove more fruitful. Furthermore, as alluded to above there is an ever-present danger that an in vitro phenotype may not hold meaning for an in vivo scenario, as the choice of culture conditions may profoundly alter the complex and flexible response of $P$. aeruginosa. An alternative approach to understanding the role of NQORs in P. aeruginosa, and the one pursued in the next chapter of this thesis, was to establish a method for exposing $P$. aeruginosa to macrophages and then recovering the viable bacteria for analysis. This also enabled the employment of microarray techniques to monitor the genome-wide transcriptional profile of PAO1 in the presence of biologically relevant oxidative stress. 


\section{Chapter 4: The $P$. aeruginosa response to challenge by murine macrophages}

\subsection{Summary}

In the previous chapter, it was determined that the native $P$. aeruginosa catalase enzyme, katA, masks protective phenotypes that NQORs may confer toward $\mathrm{H}_{2} \mathrm{O}_{2}$ exposure even though individual purified NQORs were confirmed as efficient 2electron quinone reducing enzymes with potential to mediate the quenching of reactive species like $\mathrm{H}_{2} \mathrm{O}_{2}$. Because of this we decided to take a more global approach and assess how $P$. aeruginosa interacts with immunocompetent cells which would, in an infectious environment, expose $P$. aeruginosa to $\mathrm{H}_{2} \mathrm{O}_{2}$ as well as other immune defensive compounds (e.g. cytokines, complement, etc.). We hypothesised that this would not only allow us to investigate the effects of a biologically relevant immune challenge on the expression of individual NQOR genes, it would also allow us to take a more global view of the genetic responses that $P$. aeruginosa employs to defend itself against such a challenge. This approach to infection biology is technically challenging, and albeit previously employed successfully with Salmonella, Brucella, and Mycobacterium (176-179), had not been attempted with $P$. aeruginosa prior to this work. This chapter describes the development of an infection model and method for studying $P$. aeruginosa gene expression as well as a microarray analysis of the global transcriptional events taking place during exposure to the macrophage.

\subsection{Introduction}

The development of a high-throughput infection model for assessing $P$. aeruginosa responses to challenge by immunocompetent murine macrophages was achieved using a fluorescence-based assay to quantify bacterial survival without the requirement for modifying the bacteria by insertion of reporter genes. Screening in this manner allowed for a high-volume characterization of several $P$. aeruginosa genedeleted and gene over-expression strains under different infection conditions. Results obtained were, for the most part, consistent with the biochemical assessment of 
several NQORs in Chapter 3. Finally, this infection assay was employed for transcriptional analysis of the genome by microarray.

\subsubsection{Microarray technology overview}

The development of microarray technologies has allowed for advances in genomewide transcriptional profiling, through its capacity to explore the expression of thousands of genes in a single experiment (180). In one of its most common forms, a microarray is a method by which thousands of oligo fragments are immobilized to a glass or silicon surface. Fluorophore labelled test sample cDNA fragments are then washed over the fixed oligos. Where complementary band hybridisation occurs, a measurable fluorescence intensity is emitted (Figure 4.1). The intensity measurement is then converted to a numerical value and normalized against the technical standards within the chip.

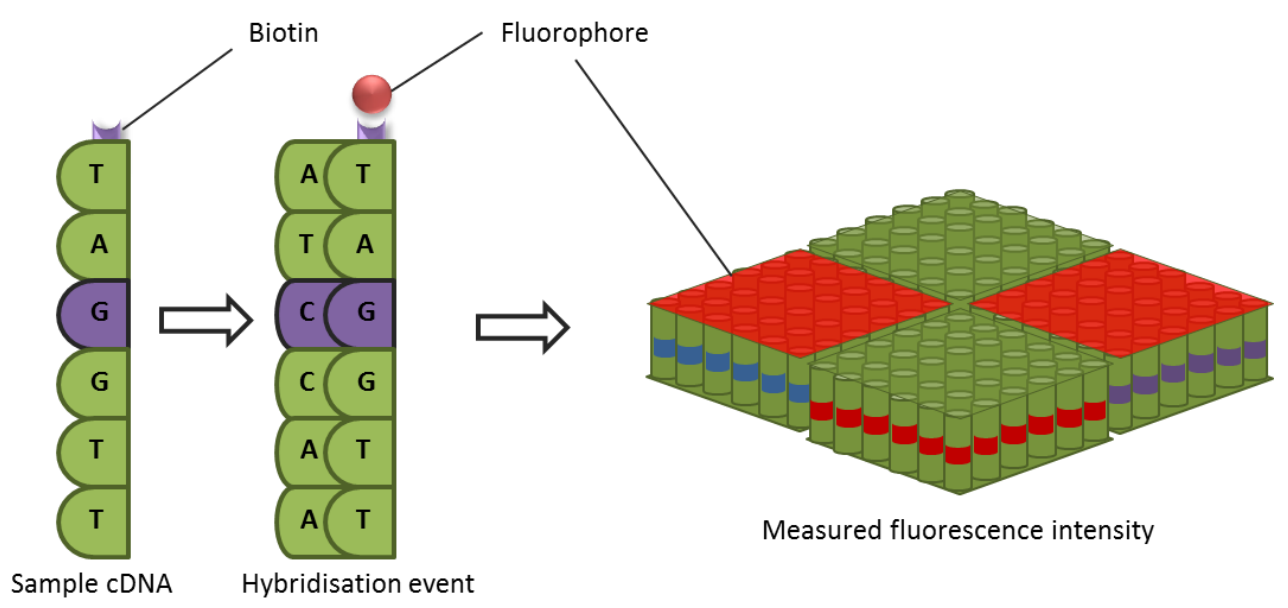

Figure 4.1. Schematic overview of Affymetrix GeneChip ${ }^{\circledR}$ technology

Multiple oligo fragments are immobilised on a glass or silicon surface. Oligos are then washed with biotinylated test CDNA and where hybridisation occurs, biotin-bound fluorophores emit measured fluorescence intensity. Recreated from Affymetrix GeneChip ${ }^{\circledR}$ Microarray Curriculum (181).

The Affymetrix Pseudomonas GeneChip ${ }^{\circledR}$ intensity array was chosen for this study based on the following characteristics: in silico probe design, a predesigned $P$. aeruginosa specific gene set, and inclusion of clinically relevant genome transcripts. The Affymetrix platform involves a high-density oligonucleotide matrix meaning that multiple biotinylated probes (10 - 20) target each gene. These probe sets for each gene 
target are selected in silico to be unique in the genome (acknowledging species specific codon usage) and non-overlapping in sequence (182). Additionally, the chip has been predesigned to represent $99.6 \%$ of the possible protein coding regions, tRNA genes, and intergenic regions of the $P$. aeruginosa laboratory strain, PAO1. The array also contains 117 genes present in $P$. aeruginosa strains other than PAO1, facilitating analysis of strains isolated from human infections (157).

Though now an accepted and widely used tool, microarray studies, if not designed properly, can still provide artificial and/or misleading results. Major sources of error in arrays can come from 1) technical variation within the chip, 2) inaccurate experimental design, or 3 ) errors in analysis (183). Because the fluorescence intensity of each probe set is assumed to be proportional to transcription, each Affymetrix probe has a corresponding mismatch probe (single base difference). By correcting for the hybridisation of the mismatch probes, non-specific events can be removed from the analysis. Though commercially prepared and processed microarrays do come with their own technical variation in probe generation and processing, through the implementation of housekeeping genes and the computational standards, microarray data can provide a robust starting point for expression profiling (184).

The applications of microbial genome arrays have been growing since their inception. Once strictly a measure of RNA transcription levels, microarrays are now a dominant tool in functional genomics not only to enable target downstream studies but to also relate molecular differences to a specific biological event, clinical strain or environmental stressor (185-187). One of the major caveats of studying bacterial transcription, however, is the instability and rapid turnover of microbial mRNAs (188192). With bacteria able to degrade expressed RNA in less than 3 minutes, and sometimes even during translation, methods of prokaryotic RNA isolation need to be highly efficient to prevent false readings of expression $(191,193-195)$. For this, microbial expression profiles should be carefully considered in the context of the experimental design.

\subsubsection{Previous microarray studies of oxidative stress in $P$. aeruginosa}

In the research domains of clinical microbiology and emerging infectious disease, transcriptional analysis of Pseudomonas spp. using the Affymetrix Pseudomonas 
GeneChip ${ }^{\circledR}$ is becoming common practice with over 240 reported studies listed in the NCBI Gene Expression Omnibus (196). The vast majority of these studies are comparative assessments of clinical Pseudomonas spp. isolated from cystic fibrosis patients. Aside from profiling clinical Pseudomonas spp., the majority of the published transcriptional research involving $P$. aeruginosa infections has been carried out using the laboratory strain PAO1.

\subsubsection{In vitro studies with oxidative stress as a constituent of culture media}

At the time of this study, three groups (Chang et al., Palma et al., and Salunke et al.) have previously published studies using transcriptional profiling of the laboratory strain PAO1 in response to challenge with exogenous $\mathrm{H}_{2} \mathrm{O}_{2}$ at varying concentrations and exposure times, as a purported model for how $P$. aeruginosa responds to oxidative challenge by mammalian immune defences (Table 4.1).

Table 4.1. Summary of PAO1 microarrays investigating oxidative stress in vitro

\begin{tabular}{|c|c|c|c|c|c|}
\hline Author & Date & Stressor & Media & Density $^{a}$ & Exposure \\
\hline Palma et al (197) & 2004 & $1 \mathrm{mM} \mathrm{H}_{2} \mathrm{O}_{2}$ & $\mathrm{TSB}^{\mathrm{b}}$ & 0.5 & $10 \min$ \\
\hline Palma et al (198) & 2005 & $0.5 \mathrm{mM} \mathrm{H}_{2} \mathrm{O}_{2}$ & $\mathrm{TSB}^{\mathrm{b}}$ & 0.5 & $10 \mathrm{~min}$ \\
\hline Chang et al (199) & 2005 & $1 \mathrm{mM} \mathrm{H}_{2} \mathrm{O}_{2}$ & LB & 0.8 & $20 \mathrm{~min}$ \\
\hline Salunkhe et al (78) & 2005 & $10 \mathrm{mM} \mathrm{H}_{2} \mathrm{O}_{2}$ & LB & $2.7-3.0$ & $2 \mathrm{~h}$ \\
\hline Goldová et al (200) & 2010 & $10 \mathrm{mM} \mathrm{H}_{2} \mathrm{O}_{2}$ & $\mathrm{Mg}^{\mathrm{c}}$ & 0.8 & $15 \mathrm{~min}$ \\
\hline
\end{tabular}

${ }^{a}$ Density is reported as an optical density at $600 \mathrm{~nm}$.

${ }^{\mathrm{b}}$ Tryptic soy broth, like LB, is a nutrient rich bacterial growth medium.

${ }^{\mathrm{C}} \mathrm{M} 9$ is a minimal growth medium.

While each project was able to justify their selection of experimental conditions, few comparable patterns were revealed between the studies. Additionally, while $\mathrm{H}_{2} \mathrm{O}_{2}$ is a major component of secreted ROS by the immune system, technical methods have not yet evolved to a point where we can appropriately quantify the concentration of $\mathrm{H}_{2} \mathrm{O}_{2}$ and other ROS compounds within the phagocytic environment. Though previous studies may have demonstrated $\mathrm{H}_{2} \mathrm{O}_{2}$ sensitivity by $P$. aeruginosa strains at distinct concentrations, these may not be relevant to a natural infection process for $P$. aeruginosa because of 1 ) the culture media, 2 ) the population density of the bacteria, and 3 ) the $\mathrm{H}_{2} \mathrm{O}_{2}$ concentration tested. For example, many of the above studies used bacteria cultured in some form of nutrient broth where, among other compounds, iron is readily available. In vertebrate systems, cells typically store iron in 
the cytoplasm thus forcing invading bacteria to develop a means of actively sequestering iron from its host to support growth (201). Similarly, it is well known that $P$. aeruginosa is sensitive to its population size and uses a density-dependent quorum sensing system to coordinate biological activities and expression of virulence factors such as biofilm formation (202).

\subsubsection{In vivo cell culture studies involving eukaryotic cell interaction with $P$. aeruginosa}

While the previous microarray work used constituents in the media to challenge PAO1, it must be appreciated that no simple system can reasonably be expected to model the complex interplay between $P$. aeruginosa and its host. As an isolated cell system may provide information regarding individual gene function, a more multifaceted approach needs to be implemented and perhaps complemented through the use of the microarray. In using the microarray to globally examine the complete transcriptome during stress responses, known relationships between genes with previously identified functions may be supported as well as identifying new target genes that give more understanding into $P$. aeruginosa-host interactions.

Frisk et al (2004; (203)) was arguably the first study to use a whole-genome transcriptional profile to assess how $P$. aeruginosa responds to eukaryotic host cells (30). During early stages of infection, $P$. aeruginosa must overcome interaction with the epithelium and find a way past the epithelial tight junctions. This study investigated the response of PAO1 (labelled with a GFP reporter gene) when exposed to airway epithelial cells. Later Chugani et al (2007; (204)) expanded upon this method by addressing PAO1 gene activation at $1 / 4$ the bacterial cell density of the Frisk et al study only to find that, for example, iron-responsive genes were differentially expressed (31).

In 2009, Alhede et al (2009; (205)) first published work investigating the interaction between PMNs and PAO1, based on the fact that PMN cells play a role in the initial host response to $P$. aeruginosa infection (32). Unlike Frisk et al and Chugani et al, Alhede's work sought to identify the gene regulation of PAO1 in a high cell density biofilm when exposed to human PMNs. The experimental design of these three studies is briefly summarised in Table 4.2. 
Table 4.2. Summary of PAO1 microarrays involving eukaryotic cell interaction

\begin{tabular}{|c|c|c|c|c|c|}
\hline Author & Date & Stressor & $\mathrm{MOI}^{a}$ & Exposure & Control \\
\hline Frisk et al (203) & 2004 & Human respiratory epithelia & $100: 1$ & $4 \mathrm{hrs}$ & DMEM \\
\hline \multirow{2}{*}{$\begin{array}{l}\text { Chugani et al } \\
(204)\end{array}$} & & & & & \multirow[t]{2}{*}{ TSB } \\
\hline & 2007 & Human respiratory epithelia & $25: 1$ & $5 \mathrm{hrs}$ & \\
\hline Alhede et al (205) & 2009 & Human PMNs & 1000:1 & $2 \mathrm{hrs}$ & $\mathrm{ABT}$ \\
\hline \multicolumn{6}{|c|}{${ }^{\mathrm{a}} \mathrm{MOI}$ refers to the number of bacteria for every eukaryotic cell; multiplicity of infection. } \\
\hline \multicolumn{6}{|c|}{$\begin{array}{l}{ }^{b} \text { Control refers to the media in which baseline samples were cultured; DMEM-F-12, a eukaryotic cell } \\
\text { culture medium containing } 0.4 \% \text { glucose, } 13 \mu \mathrm{M} \mathrm{Fe}^{2 / 3+}, 2 \mathrm{mM} \text { L-glutamine, } 15 \mathrm{mM} \mathrm{HEPES} \text {, and } 2 \%\end{array}$} \\
\hline
\end{tabular}

Although PMNs are more abundant in the blood, macrophages naturally reside at the epithelial interface in normal lung tissue, produce more ROS, and play an essential role in killing and controlling attacking bacterial pathogens (206-212). Based on this, we sought to develop a means to examine the PAO1 in the presence of macrophages. We focused on studying the initial macrophage phagocytic interaction that infecting non-mucoid $P$. aeruginosa may encounter in the early stages of pathogenesis.

Our approach incorporated the use of an in vivo cell culture model whereby viable bacteria were engulfed by macrophages and then isolated and assessed for survival or transcriptional response. The term "in vivo" is this case was used to indicate that the bacteria were in an environment different from in vitro LB cultures even though this model is still an "in vitro" model for the macrophages. All instances of the term "in vivo cell culture" refer to this infection model. Though the laboratory strain PAO1 has been highly passaged at a potential cost to its virulence (213), we chose to employ the PAO1 strain so that we could directly compare to transcriptional data from the above mentioned in vitro studies (Table 4.1.); and, unlike the earlier Frisk et al study (203) we chose not to incorporate a GFP tag, to keep the PAO1 as close to wild type as possible and thereby avoid any perturbation of standard transcriptional profiles arising from accumulation of non-native proteins in the cell. To achieve this it was first necessary to develop a method to isolate RNA from live $P$. aeruginosa located within the macrophage. Similar studies with Salmonella, Brucella, and Mycobacterium have indicated that this approach can be highly effective in identifying oxidative stressresponsive genes and enzymes (176-179); however, equivalent experiments had not previously been described for $P$. aeruginosa. 
In Chapter $3 P$. aeruginosa was identified as possessing numerous NQORs, most of which have not been experimentally characterised with regard to their biological role, and our results did not definitively identify whether these enzymes contribute to oxidative stress resistance. To further investigate this, the work described in this chapter sought to identify, at a global level, which genes or gene-groups in $P$. aeruginosa are activated in response to incubation with macrophage cells (the initial immune system responders to bacterial infection, which fight bacteria by releasing a transient "oxidative burst"). This analysis was conducted by using Affymetrix microarray technology to compare the gene expression of $P$. aeruginosa cells that have been incubated with macrophages against that of control cells which have not been exposed to oxidative stress $(214,215)$. As well as accumulating evidence for NQORs that may play a role in virulence, this study aimed to provide a global perspective on how this important human pathogen responds to the initial challenge of the human immune system.

\subsection{Objectives}

- Develop a macrophage infection model using flow cytometry to quantify survival in the absence of a GFP reporter system.

- Screen $P$. aeruginosa strains using in vivo cell culture infection model.

- Assess transcriptional profile of $P$. aeruginosa internalised by macrophages. 


\subsection{Development of an in vivo cell culture infection model to evaluate the $\boldsymbol{P}$. aeruginosa response to challenge by murine macrophages}

\subsubsection{Using flow cytometry to quantify bacteria}

A standard means of quantifying bacterial survival is to use colony forming unit (CFU) counts of samples exposed to different conditions. This approach, although effective, is low throughput and was not practical for the volumes of strains and conditions included in this study. We therefore sought to develop a more rapid means of quantifying $P$. aeruginosa survival. To do so, we adapted the Bacterial Counting Kit (Molecular Probes, Life Technologies; Grand Island, NY, USA) for use with $P$. aeruginosa cultures and optimised a protocol for assessing $P$. aeruginosa viability using flow cytometry. In short, the Bacteria Counting Kit incorporates a live cell dye (SYTO ${ }^{\circledR}$ $\mathrm{BC}$ ) and microsphere bead standard used in conjunction with propidium iodide ( $\mathrm{PI}$; a "dead cell" stain). SYTO ${ }^{\circledR}$ BC is a cell wall permeable nucleic acid stain that produces a bright green fluorescence in viable cell populations. In contrast, $\mathrm{PI}$ is not cell permeable, and is therefore commonly used to identify dead cell populations in the red-fluorescence channel, allowing differentiation between live and dead cells within a mixed population. The kit includes microsphere standards that are also fluorescently labelled and can be used to quantify the bacteria by determining the ratio of bacterial signals to microsphere signals.

Fluorescence activated cell sorting (FACS) was initially employed to confirm that the cytometric populations indicative of positive $\mathrm{SYTO}^{\circledR} \mathrm{BC}$ staining accurately identified the live bacterial population. Cell sorting was performed by Kylie Price at the Malaghan Institute for Medical Research, such that each cell population (separated by quadrant, Figure 4.2) was collected for recovery on agar and in liquid culture. Although there was overlap in signal from SYTO ${ }^{\circledR} \mathrm{BC}$ and PI populations (quadrant 2 and 3), it was confirmed by CFU counting that these "double positive" populations were indeed representative of a live cell group (Figure 4.2b). 

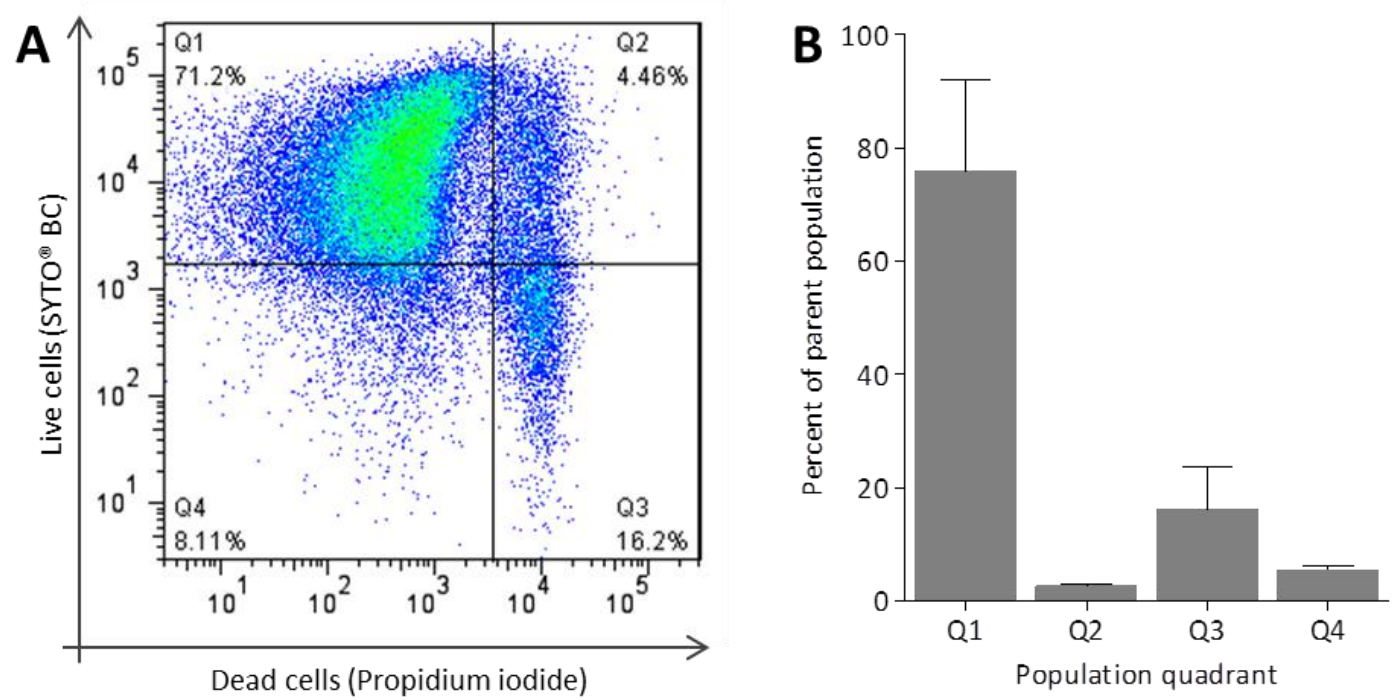

Figure 4.2. $P$. aeruginosa quantification by flow cytometry and CFU

(A) Cytogram representation of PBS-washed PAO1 stained with both $1 \times$ SYTO ${ }^{\circledR}$ BC and $10 \mu \mathrm{g} / \mathrm{mL} \mathrm{PI} \mathrm{in}$ $0.9 \% \mathrm{NaCl}$ and processed on a FACSVantage DiVa cell sorter where quadrant Q1 theoretically represents $\mathrm{SYTO}^{\circledR} \mathrm{BC}+\mathrm{PI}$ - cells, Q2 and Q3 the double positive bacteria, and Q4 the non-labelled bacteria. (B) PAO1 populations from each quadrant were collected to sterile polystyrene tubes containing $5 \mathrm{~mL} 5 \% \mathrm{FCS}$, $0.9 \% \mathrm{NaCl}$. The bacteria were then harvested by centrifugation (4000 $\mathrm{x}$, $4 \mathrm{~min}$, room temperature) and quantified by serial dilution on solid agar for CFU counts. Cell sorting was performed twice with consistent results. This figure presents representative data for (A) one cell sort, and (B) the CFU counts performed in quadruplicate \pm 1 standard deviation.

Quantification via CFU counts post cell sorting validated that the Bacteria Counting Kit could indeed be used to measure $P$. aeruginosa survival. However, due to differences in laser strength and signal output between the commercial cell sorter (FACSVantage DiVa) and the laboratory benchtop analyser (FAC FACSCanto II), two modifications were made to the viable population selection gating on the cytogram. Firstly, because Q2 and Q3 from the cell sort (Figure 4.2A) both appeared to be viable populations, we found that increasing the PI concentration from $10 \mu \mathrm{g} / \mathrm{mL}$ to $20 \mu \mathrm{g} / \mathrm{mL}$ reduced non-specific binding of $\mathrm{PI}$ (commonly observed with $\mathrm{PI}$ in the presence of Syto ${ }^{\circledR}$ dyes; (216-219)) and therefore eliminated the Q3 “double positive" population (data not shown). (216)Secondly, the cytogram population gating methods were adjusted to accommodate increased laser noise associated with routine flow cytometry analysis on a benchtop analyser (complete gating strategy description in Section 2.14.1). The final, optimised, flow cytometry staining solution used in this study consisted of $1 \times$ SYTO $^{\circledR} \mathrm{BC}, 1 \times\left(10^{6}\right)$ microsphere beads, and $20 \mu \mathrm{g} / \mathrm{mL} \mathrm{PI}$ in $0.9 \%$ $\mathrm{NaCl}$. 
To validate this gating protocol, overnight cultures of $P$. aeruginosa were diluted 1:10 in fresh LB and incubated for a further $4 \mathrm{~h}$ before being washed twice in PBS. Washed bacteria were then incubated in either PBS (Live) or $70 \%$ isopropanol (Dead) for $30 \mathrm{~min}$ at room temperature before being washed again in PBS. Triplicates of each sample were then a) serially diluted then plated on LB agar, or b) stained with the flow cytometry staining solution and enumerated using flow cytometry. After normalising the flow count to the bead standards within the Bacteria Counting Kit, the number of bacteria recorded was sufficiently within the standard error of each quantification method (Figure 4.3). This was repeated, with similar results, on multiple occasions.

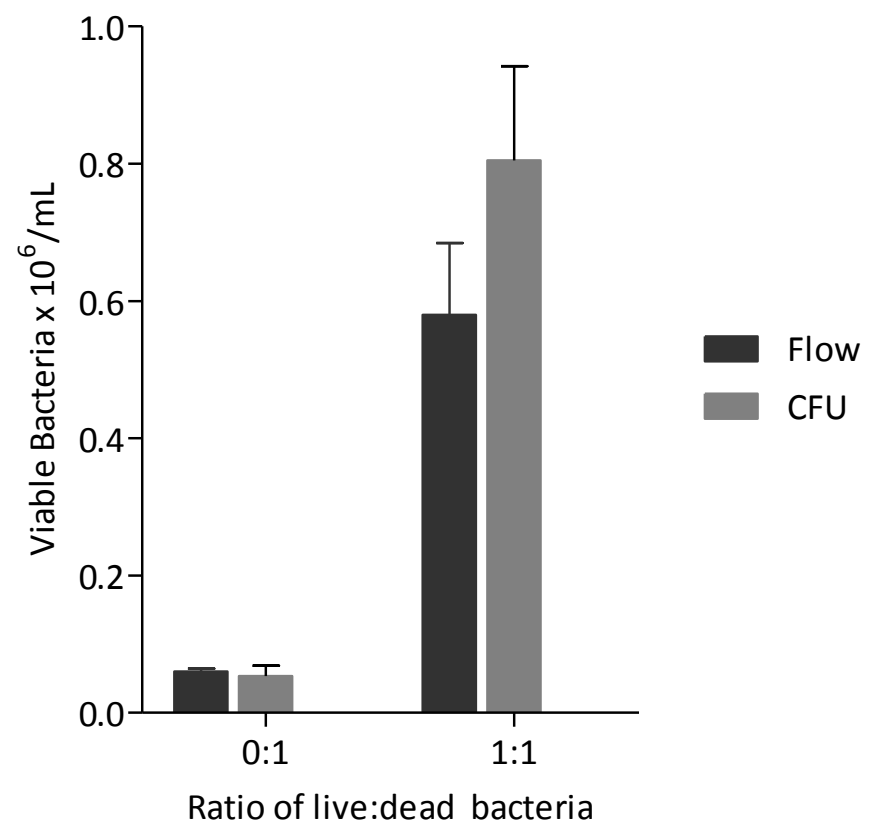

Figure 4.3. Live and dead PAO1 quantification by flow cytometry versus CFU counts Live and dead cell controls were prepared in triplicate by washing overnight cultures of PAO1 twice in PBS then dividing each sample into two replicates. One of these was untreated PAO1 in PBS ("live"), the other PAO1 in 70\% isopropanol ("dead") for $30 \mathrm{~min}$ at room temperature. After incubation, both live and dead cell samples were washed another two times in PBS, combined at ratios of 0:1 and 1:1 (live to dead), stained with the optimised flow cytometry staining solution, and either collected on the FACSCanto II flow cytometer or serially diluted on LB agar for CFU counts. The quantification of flow events was achieved through an optimised flow gating strategy (Section 2.14.1) or by standard CFU calculations and plotted as the average of triplicate samples \pm 1 standard deviation. 


\subsubsection{Proof of Principle: Using flow cytometry and confocal microscopy to quantify antimicrobial properties of nanoparticle-coated wool}

During the optimisation phase of our flow cytometry protocol the opportunity arose to collaborate with Professor Jim Johnston and his team of chemists at VUW, seeking to evaluate the antibacterial properties of wool that they had coated with immobilized palladium nanoparticles. Due to surface plasmon resonance effects, the immobilized nanoparticles confer unusual, attractive and permanent coloration to the wool, as well as an expectation of antimicrobial effects. To assist with their study we proposed that using flow cytometry to enumerate the proportion of viable $E$. coli cells remaining after their standard test (a seven-day room temperature incubation of bacteria that had been washed and resuspended in PBS then adsorbed onto the surface of different wool samples) might prove a far more rapid and equally reliable method of quantification than plating dilution series of bacteria and counting the CFUs.

To test this, the antimicrobial properties of identical preparations of nanoparticle-coated wool were quantified using either (i) conventional CFU measurements; or (ii) flow cytometry, backed up with qualitative analysis by confocal microscopy. Wool was loaded with nanopalladium particles grown at different temperatures ranging from $23{ }^{\circ} \mathrm{C}$ to $100{ }^{\circ} \mathrm{C}$ where temperature directly regulates particle size: the lower the temperature, the bigger the particle (220). We hypothesised that larger sized particles (prepared at $23{ }^{\circ} \mathrm{C}$ ) would have more surface area available and therefore more palladium available for bacterial killing. Samples of wool, prepared under different nanoparticle growth conditions, were subjected to the standard E. coli incubation test as described above, following which the bacteria were collected and assessed for survival by both CFU and flow cytometry measurements (Figure 4.4A). The bacteria stained with the flow cytometry staining solution were also subjected to qualitative assessment using scanning laser confocal microscopy (Figure 4.4B). The survival imaging of nanopalladium wool-challenged $E$. coli was shown to qualitatively reflect CFU / flow cytometry enumeration where sample B and C were most viable and $E$ was least viable post treatment. A detailed summary of the complete study is presented in Appendix A1. 

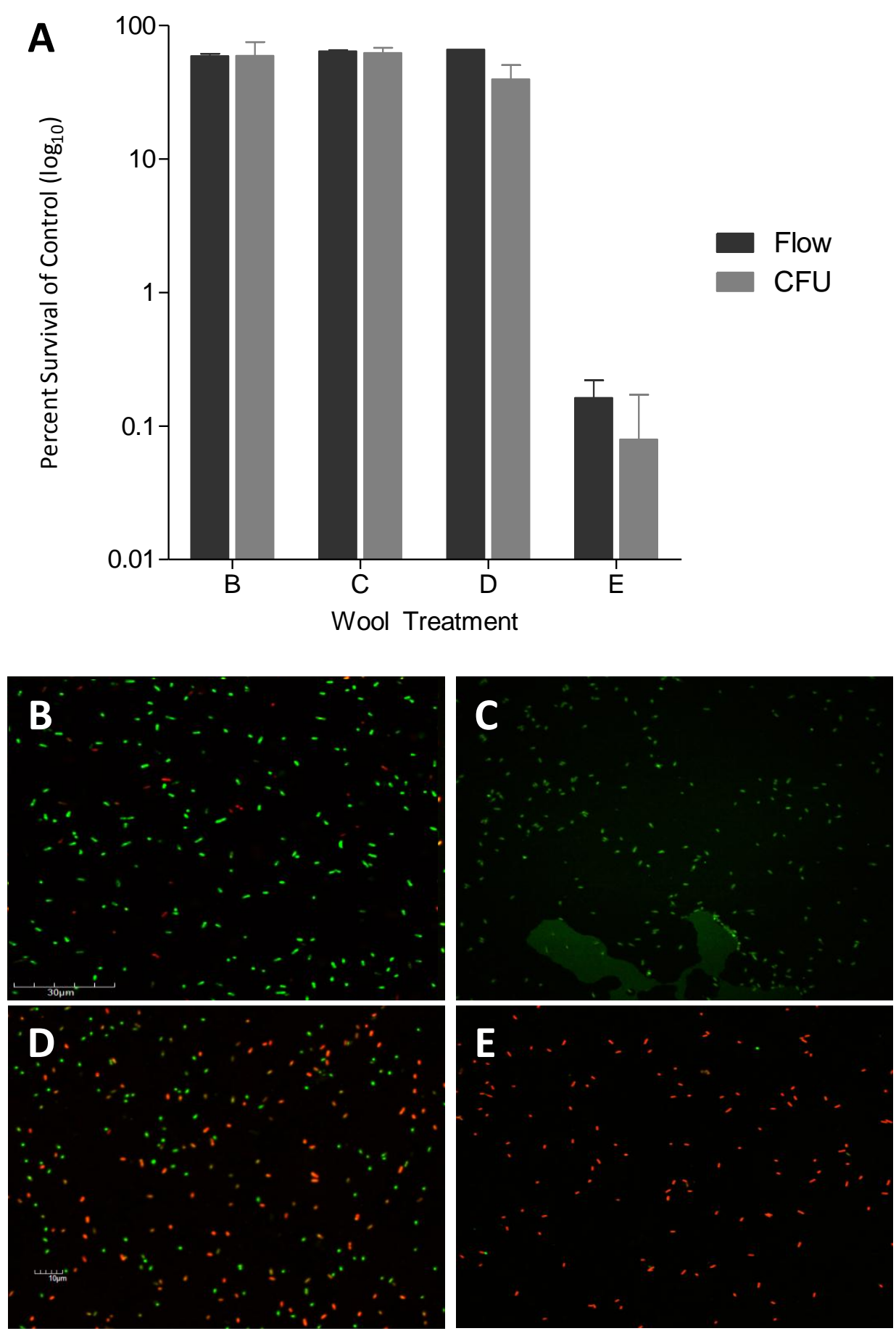

\section{Figure 4.4. Bacterial survival assessment by flow cytometry, CFU and confocal}

\section{microscopy}

(A) Identical samples of $E$. coli W3110 were recovered from nanopalladium coated merino wool, stained with $1 \times$ SYTO $^{\circledR} \mathrm{BC}$ and $10 \mu \mathrm{g} / \mathrm{mL} \mathrm{PI}$ in $0.9 \% \mathrm{NaCl}$, and quantified by either flow cytometry (grey bars) or CFU counts (black bars). Wool treatments $\mathrm{B}-\mathrm{E}$ represent different conditions whereby nanoparticles were immobilised on the wool fibres $\left(100^{\circ} \mathrm{C}, 80^{\circ} \mathrm{C}, 50^{\circ} \mathrm{C}\right.$, and $23^{\circ} \mathrm{C}$ respectively). The data presented is a representative of three experimental replicates and error bars depict \pm 1 standard deviation. (B-E) Corresponding confocal microscopy images of wool-treated W3110 samples were obtained by fixing the stained bacteria (as above) in 2\% (final) paraformaldehyde, PBS and acquiring images by a laser-scanning confocal microscope (FlowView ${ }^{\circledast}$ FV1000, Olympus). Samples were excited sequentially at 473 and 559 $\mathrm{nm}$. Images were processed using Olympus FV10-ASW Viewer Ver.2.0b. 
The reproducibility of the two quantitative methods was high, with no statistical difference between them. Therefore flow cytometry was deemed an acceptable means of bacterial quantification and appropriate for the higher throughput application of assessing viability of bacterial strains in different treatment conditions. In developing the infection model, initial $P$. aeruginosa cultures were also quantified using both methods to confirm that $P$. aeruginosa, like $E$. coli in the wool study, could be reliably quantified with flow cytometry.

\subsubsection{Collection method of macrophage-engulfed $P$. aeruginosa}

Having shown that flow cytometry could be used to accurately quantify bacterial viability, we next sought to develop a means for recovering viable, non-GFP labelled $P$. aeruginosa for assessment. We sought to utilise established murine macrophages for initial method optimisation. For one, the immortal cell lines would provide ease of proliferation and maintenance versus primary cell lines while still exhibiting classic phagocytic and macrophage-like characteristics (221-223). Secondly, the repeated optimisation steps likely to be required would benefit from using genetically identical progeny of the immortalised cell lines. The intended microarray study had a technical requirement of $30 \mu \mathrm{g}$ of RNA for each test sample (10 $\mu \mathrm{g}$ for each of three replicates) in the microarray application. Therefore, the murine macrophage line was chosen based on the ability to recover the greatest number of viable $P$. aeruginosa postphagocytosis.

To achieve this we adapted protocols from multiple sources (Eriksson et al (2003; (176)), Srivastava et al (2007; (178)), and Dietrich et al (2000; (179)) that had previously employed related techniques to study Salmonella and Mycobacterium spp. The key concept was to allow enough time for macrophages to phagocytose $P$. aeruginosa and produce ROS, during which time the bacteria located externally to the macrophage would be removed by antibiotic treatment and washing. Subsequently, the bacteria-containing phagosomes would then be allowed to mature before lysis of the macrophages to harvest viable $P$. aeruginosa.

To achieve this required the optimisation of a number of parameters. In short, the initial infection conditions tested were as follows. Macrophage-like RAW-264.7 or J774A.1 cells were seeded at $8.4 \times 10^{3}$ per well in antibiotic-free complete cell culture 
media (CMEM; 25 mM HEPES Buffer solution, 10\% FCS in basal media Gibco ${ }^{\circledR}$ DMEM; Invitrogen) and incubated at $37^{\circ} \mathrm{C}, 5 \% \mathrm{CO}_{2}$ for $48 \mathrm{~h}$. Overnight cultures of PAO1 in antibiotic-free LB were washed twice in room temperature PBS and diluted to $\mathrm{OD}_{660}$ 1.0 which equates to roughly $1 \times 10^{9}$ bacteria/mL (based on multiple preliminary serial dilution tests, data not shown). A PAO1 multiplicity of infection (MOI) of 100 (i.e. 100 bacteria: 1 macrophage in $100 \mu \mathrm{l}$ per well) in complete CMEM was then applied to a PBS-washed macrophage monolayer. To promote bacteria-macrophage interaction, the culture plates were centrifuged at $1000 \mathrm{x} g$ for $2 \mathrm{~min}$ at room temperature after addition of bacteria-containing media and before commencing a $2 \mathrm{~h}$ infection period at $37^{\circ} \mathrm{C}, 5 \% \mathrm{CO}_{2}$. After infection, gentamycin was added to the CMEM (final concentration $50 \mu \mathrm{g} / \mathrm{mL}$ ) for $45 \mathrm{~min}$ to kill the non-engulfed $P$. aeruginosa. This concentration was sufficient to kill $>98 \%$ of $P$. aeruginosa while having no deleterious effect on macrophage viability (Figure 4.5)
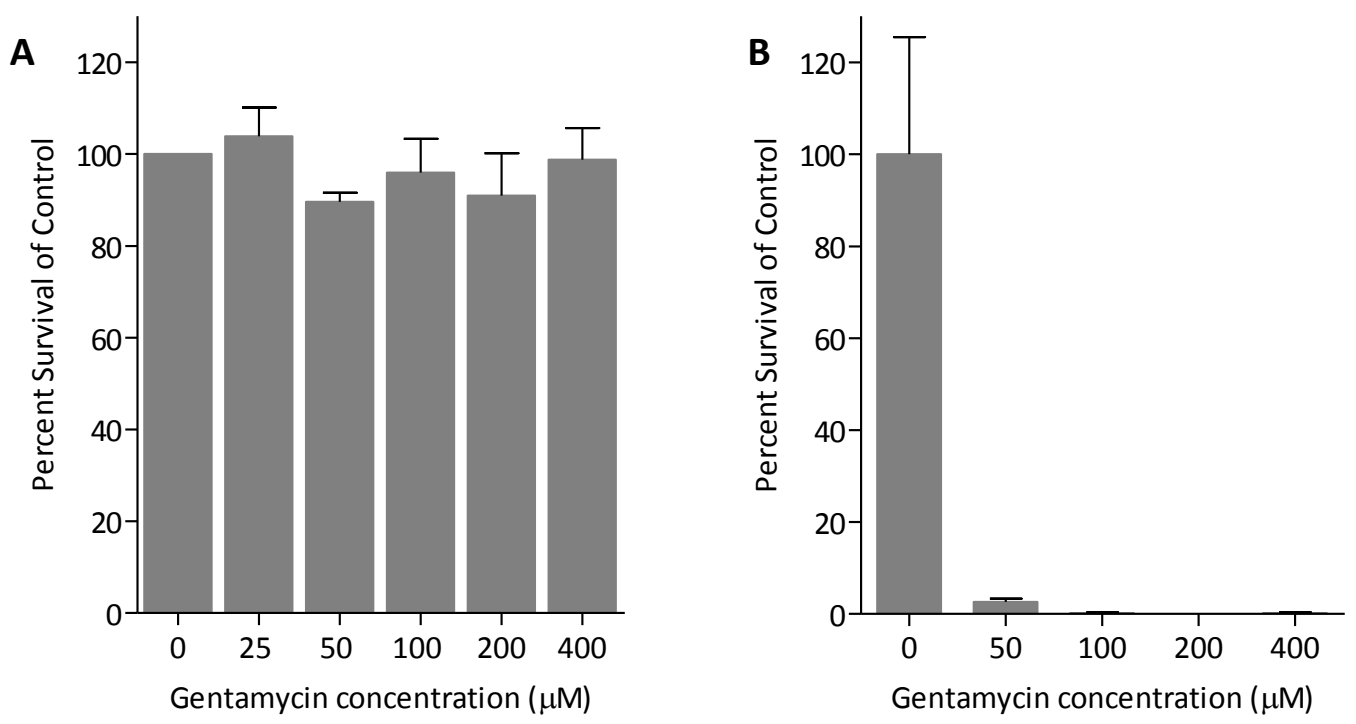

Figure 4.5. Gentamycin protection assay

Replicate cultures of RAW-264.7 macrophages (A) and PAO1 (B) were treated for $45 \mathrm{~min}$ at a range of concentrations of gentamycin $(0 \mu \mathrm{M}-400 \mu \mathrm{M})$ in $\mathrm{CMEM}$ at $37^{\circ} \mathrm{C}, 5 \% \mathrm{CO}_{2}$ and assessed for survival using flow cytometry. Both macrophages and PAO1 were stained with the flow cytometry staining solution ( $1 \times \mathrm{SYTO}^{\circledast} \mathrm{BC}, 20 \mathrm{\mu g} / \mathrm{mL} \mathrm{PI}, 1 \times$ microsphere beads in $0.9 \% \mathrm{NaCl}$ ). The $\mathrm{SYTO}^{\oplus} \mathrm{BC}$ dye is a nucleic acid dye that can stain either eukaryotic or prokaryotic cells but at different fluorescent intensities allowing for both cell types to be assessed. Data is indicative of three replicates \pm 1 standard deviation. Similar observations were made with gentamycin-treated J774A.1 macrophages (data not shown).

After gentamycin treatment, infected macrophage monolayers were washed four times in PBS to remove the extracellular P. aeruginosa. This point equated to time point zero (TO). To subsequently release the internalised bacteria at different time 
points, washed monolayers were lysed for $5 \mathrm{~min}$ in macrophage lysis solution $(0.1 \%$ SDS, $0.1 \%$ Tween-20, $0.9 \% \mathrm{NaCl}$ ). The lysate was then removed in 2 vol PBS and transferred to a fresh 96-well plate. The bacteria were then collected by differential centrifugation, first spinning at 400x $g$ for 4 min to pellet the macrophage cellular debris, then by transferring the bacteria-containing supernatant to a new 96 -well plate and spinning at $4000 \times \mathrm{g}$ for $4 \mathrm{~min}$ to pellet the bacteria. The bacterial pellet was then resuspended in flow cytometry staining solution and incubated in the dark at room temperature for 15 min before being assessed for viability by flow cytometry.

Initially, established RAW-264.7 and J774A.1 macrophage-like cell lines were compared for their ability to internalise $P$. aeruginosa at different $\mathrm{MOI}$ from $5-200$ bacteria per macrophage. Irrespective of the MOI used, a greater number of viable $P$. aeruginosa cells were recovered from the RAW-264.7 than the J774A.1 cells (Figure 4.6). On this basis RAW-264.7 macrophages were chosen for infection model development.

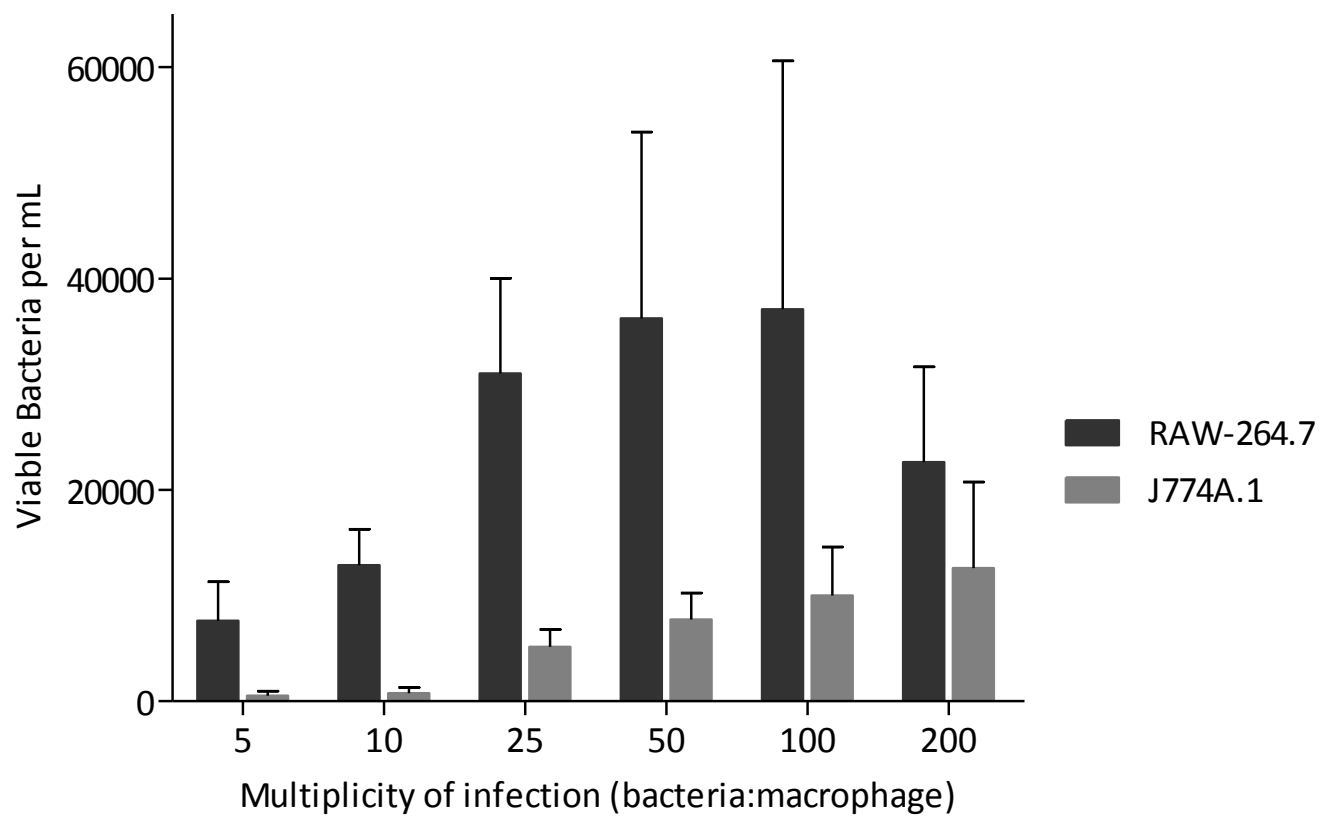

Figure 4.6. Viable P. aeruginosa recovered from RAW-264.7 and J774A.1

\section{macrophages}

Replicate cultures of RAW-264.7 or J774A.1 macrophages were infected for $2 \mathrm{~h}$ with PBS-washed $P$. aeruginosa resuspended in pre-warmed CMEM at MOIs of 5-200. Infection was followed by a $100 \mu \mathrm{g} / \mathrm{mL}$ gentamycin treatment for $45 \mathrm{~min}$. The infected macrophages were washed free of externally located bacteria. The internalised bacteria were then recovered from infected macrophages by application of macrophage lysis solution and resuspension of the pellet from the second stage of differential centrifugation (400x $g$ followed by $4000 \times g$ for $4 \mathrm{~min}$ ). Viable bacteria were then stained with flow cytometry staining solution and quantified using flow cytometry, measuring the ratio of bacteria signals to Bacteria Counting Kit bead signals. The data presented in this graph indicates the mean of three independent experiments \pm 1 standard deviation. 
Preliminary tests indicated no significant statistical differences in the number of viable bacteria recovered from RAW-264.7 macrophages at MOls of 25, 50 and 100; the latter was chosen for this assay because more total prokaryotic RNA was collected at a $\mathrm{MOI}$ of 100 than at a $\mathrm{MOI}$ of 50 according to the percent total area as computationally predicted by the Agilent 2100 Bioanalyzer software (Agilent Technologies; Santa Clara, CA, USA; Figure 4.7).
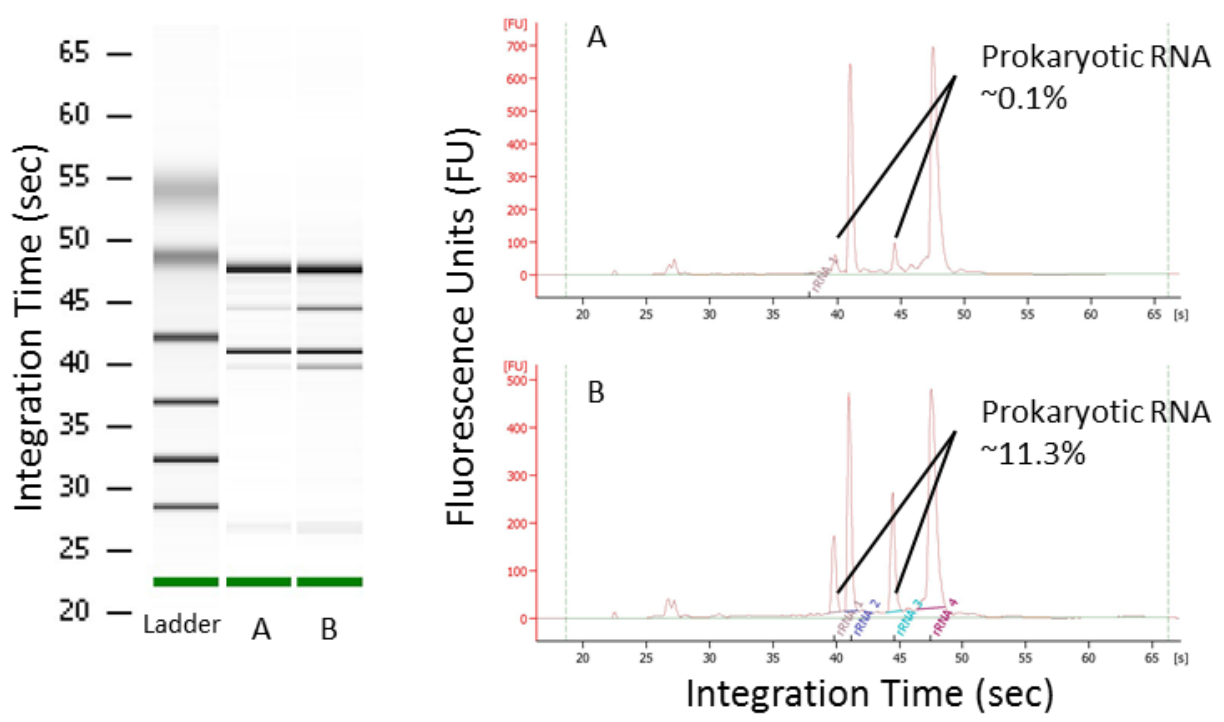

Figure 4.7. Total prokaryotic RNA recovered from RAW-264.7 macrophage-engulfed

\section{$P$. aeruginosa}

Total RNA was recovered by application of $1 \mathrm{~mL}$ Trizol to RAW-264.7 macrophages infected for $2 \mathrm{~h}$ by a PAO1 MOI of 50 (A) and a MOI of 100 (B). After total RNA purification (manufacturer's specifications; QIAGEN RNAeasy Kit), prokaryotic RNA was then enriched using the MICROBEnrich ${ }^{\text {TM }}$ kit with the recommended protocol (Ambion ${ }^{\circledR}$, Life Technologies) and quantified using the Agilent 2100 Bioanalyzer. The enriched bacterial RNA represented by virtual gel (left) or electropherogram (right), showed band/peaks corresponding to the eukaryotic $18 \mathrm{~S}$ and $28 \mathrm{~S}$ and prokaryotic $16 \mathrm{~S}$ and $23 \mathrm{~S}$ ribosomal subunits. RNA abundance of prokaryotic RNA was calculated as the $16 \mathrm{~S}$ plus $23 \mathrm{~S}$ area under the curve as a percentage of total eukaryotic plus prokaryotic surface area. This was repeated in two separate experiments in duplication. This figure presents data from one replicate.

Additionally, internalisation of $P$. aeruginosa by RAW-264.7 macrophages was visually confirmed by confocal microscopy using both SYTO ${ }^{\circledR} \mathrm{BC}$ from the Bacteria Counting Kit and the Image-IT ${ }^{\circledR}$ LIVE Plasma Membrane and Nuclear Labelling Kit Counterstains For GFP-Expressing Cells (Molecular Probes). Pre-infection staining of the bacteria in concentrated $\mathrm{SYTO}^{\circledR} \mathrm{BC}(2 \mathrm{x}$ in $0.9 \% \mathrm{NaCl})$ allowed internalised PAO1 to be visually confirmed via green fluorescence compared to non-infected controls (Figure 4.8). However, because $\mathrm{SYTO}^{\circledR} \mathrm{BC}$ is a permeable nucleic acid dye, background levels of dye were also visible in the cytoplasm of the infected macrophages. 

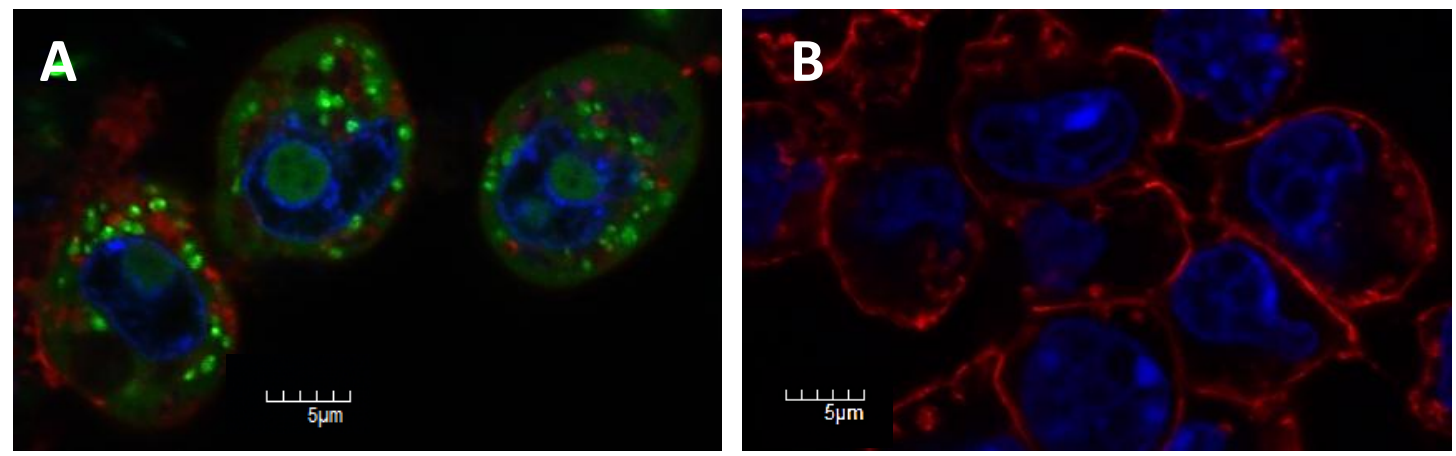

Figure 4.8. Confocal images of RAW-264.7 infected with $P$. aeruginosa

(A) Prior to macrophage infection, $1 \mathrm{~mL}$ of PBS-washed $P$. aeruginosa cultures were pelleted by centrifugation at 10,000 $\mathrm{g}$ for $30 \mathrm{~s}$ and resuspended in 1:500 in 0.9\% $\mathrm{NaCl}$. After staining with SYTO ${ }^{\circledR} \mathrm{BC}$ for $15 \mathrm{~min}$ at room temperature in the dark, the bacteria were diluted to a $\mathrm{MOI}$ of $100 \mathrm{in} 2 \mathrm{~mL}$ prewarmed, phenol red-free CMEM and applied to the macrophage monolayer seeded in a $35 \mathrm{~mm}$ glassbottom FluoroDish ${ }^{\mathrm{TM}}$ cell culture dish (World Precision Instruments). After $2 \mathrm{~h}$ infection followed by 100 $\mu \mathrm{g} / \mathrm{mL}$ gentamycin treatment for $45 \mathrm{~min}$, extracellular bacteria were washed free of the monolayer in PBS and the remaining RAW-264.7 monolayer was then stained with $5 \mu \mathrm{g} / \mathrm{mL}$ Alexa Fluor 594 WGA and $1 \mu \mathrm{M}$ Hoechst 33342 in $1 \mathrm{~mL}$ of phenol red-free CMEM for $10 \mathrm{~min}$ at $37{ }^{\circ} \mathrm{C}, 5 \% \mathrm{CO}_{2}$. Stained live-cell cultures were washed three times in PBS, covered in $1 \mathrm{~mL}$ of phenol red-free CMEM and imaged using a laser-scanning confocal microscope (FlowView ${ }^{\circledR}$ FV1000, Olympus). (B) Uninfected RAW-2654.7 cells were prepared by washing the monolayer in PBS and staining with Alexa Fluor 594 WGA and Hoechst 33342 as above. All samples were excited sequentially at 405, 473 and $559 \mathrm{~nm}$. Images were processed using Olympus FV10-ASW Viewer v2.0b.

\subsubsection{Optimisation of the infection environment}

In a hypothetical infection scenario, human subjects would almost certainly have been previously exposed to $P$. aeruginosa at some point in their lives; as such, bacteria would naturally be opsonised (coated by complement proteins for macrophage recognition) upon entry into the system. We therefore decided to opsonise the $P$. aeruginosa cells prior to macrophage challenge, unless this was found to lead to a substantial reduction in the total number of cells recovered post-internalisation. In this experiment, opsonisation was found to have little effect on the number of viable $P$. aeruginosa recovered (Figure 4.9).

Another common treatment is stimulation of macrophage cultures by addition of interferon-gamma (INF-y) and lipopolysaccharide (LPS) prior to application in an immunological assay. However, in the context of this in vivo cell culture model, the influence of priming RAW-264.7 cells with INF- $\gamma$ caused a significant reduction in the number of $P$. aeruginosa recovered and this pre-treatment was therefore not used in subsequent assays (Figure 4.9). 


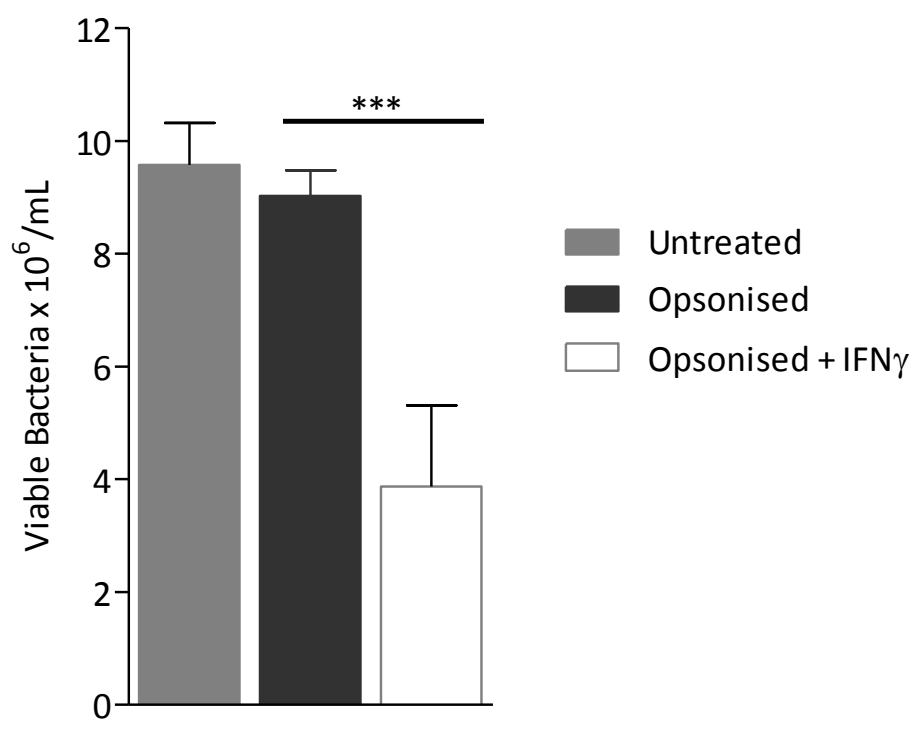

Figure 4.9. Effect of opsonisation and INF-y on P. aeruginosa uptake

RAW- 264.7 macrophages with or without $100 \mathrm{U} / \mathrm{mL}$ IFN-y pre-treatment for $24 \mathrm{~h}$ were infected with either untreated or opsonised $P$. aeruginosa for $2 \mathrm{~h}$. Opsonised bacteria were prepared by resuspending PBS-washed PAO1 in $10 \% \mathrm{C} 57 / \mathrm{BL} 6$ male mouse serum PBS for 30 min at $37{ }^{\circ} \mathrm{C}, 5 \% \mathrm{CO}_{2}$ before diluting to a $\mathrm{MOI}$ of 100 in pre-warmed CMEM for application to the macrophage monolayer. Infection was followed by a $100 \mu \mathrm{g} / \mathrm{mL}$ gentamycin treatment for $45 \mathrm{~min}$. The infected macrophages were then washed free of externally located bacteria and the internalised bacteria. The bacteria were then recovered from infected macrophages by application of macrophage lysis solution and differential centrifugation at $400 \times g(4 \mathrm{~min})$ then $4000 \times g(4 \mathrm{~min})$. Bacteria were then stained with flow cytometry staining solution and the number of viable cells quantified using the ratio of bacteria signals to Bacteria Counting Kit bead signal, measured by flow cytometry. Plotted data indicate the mean data from three independent experiments \pm 1 standard deviation.

After confirming that (i) P. aeruginosa was being internalised by RAW-264.7 macrophages, (ii) viable bacteria could be recovered and quantified post infection, and (iii) pre-infection priming with INF- $\gamma$ was unnecessary (and indeed counter-productive), the next step in method development was to determine the time point at which internalised bacteria would ultimately be isolated to maximise RNA isolation. For this, bacteria from infected RAW-264.7 macrophage replicates were recovered at a range of different times $(0,2,4,8$ and $24 \mathrm{~h})$ post infection. As noted previously, time $0 \mathrm{~h}$ (T0) was taken as the point immediately after $2 \mathrm{~h}$ infection and 45 min gentamycin treatment (T0) which allows the macrophage phagosome to mature (e.g. a series of events ultimately leading to the formation of the phagolysosome), and which can dramatically change the environment to which bacteria are exposed. Phagosomal maturation leading to lysosomal fusion has previously been reported to complete 
between 60 minutes and 4 hours post phagocytosis depending on the infection conditions $(176,224-228)$.

Initial results indicated a significant reduction in the number of viable bacteria between T0 and after $2 \mathrm{~h}$ of phagosomal maturation and even more so at $8 \mathrm{~h}$. However, if allowed to incubate for a total of $24 \mathrm{~h}$, the wild type PAO1 P. aeruginosa was able to recover to near T0 levels (Figure 4.10). PAO1 lacking the KatA catalase $(\Delta k a t A)$ was also tested for viability during phagosomal maturation in tandem with wild type PAO1. Consistent with the in vitro assays in Chapter $3, \Delta k a t A$ was unable to survive infection as effectively as wild type at T0. However, like wild type, $\Delta k a t A$ was able to recover after $24 \mathrm{~h}$ (Figure 4.10). Given the detrimental effect of the subsequent stages of phagosomal maturation on PAO1 viability and our initial goal to investigate early-stage macrophage interactions, the T0 time point was chosen for this study.

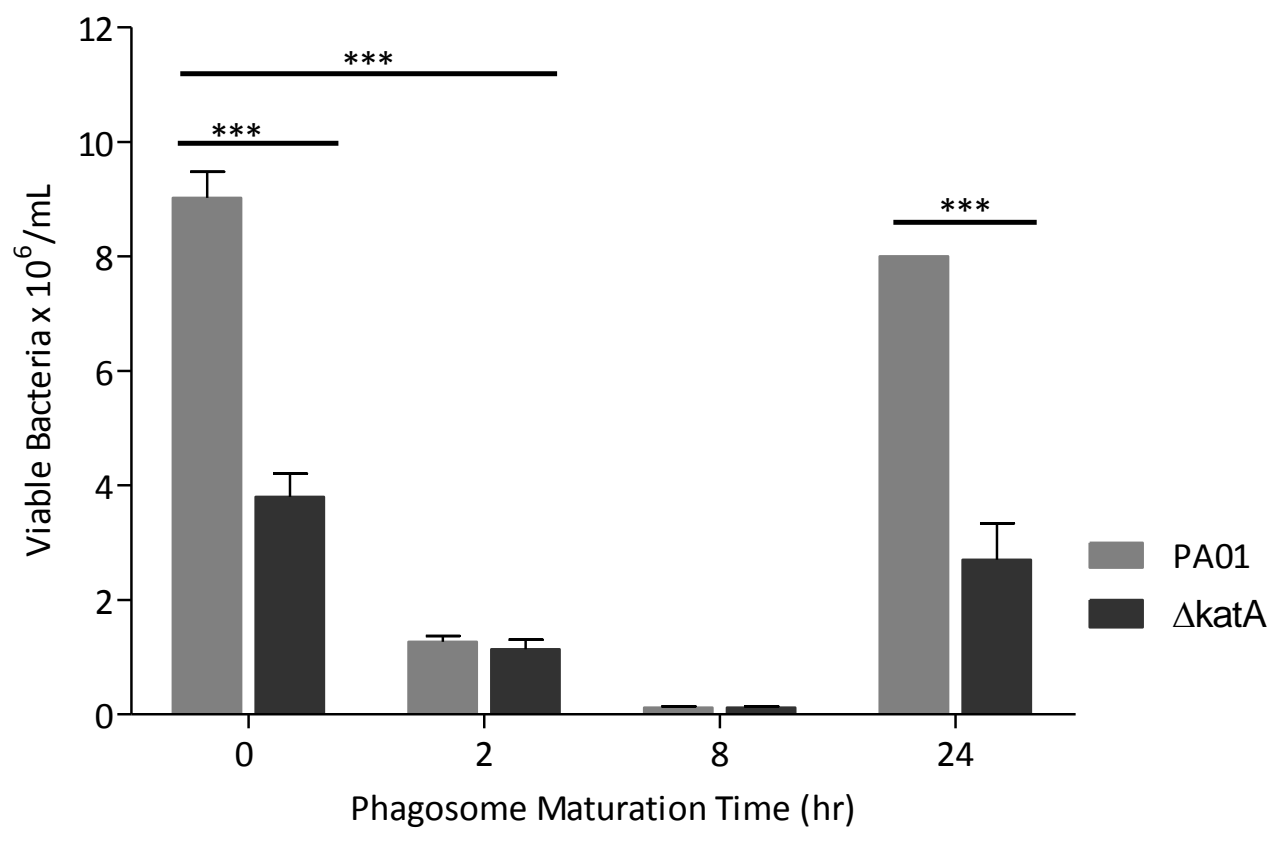

Figure 4.10. Recovery of viable $\boldsymbol{P}$. aeruginosa post-phagosomal maturation RAW-264.7 macrophages were infected for $2 \mathrm{~h}$ with a MOI of 100 of opsonised wild type PAO1 or opsonised $\Delta k a t A$-deletion mutant followed by a $100 \mu \mathrm{g} / \mathrm{mL}$ gentamycin treatment for $45 \mathrm{~min}$. The infected macrophages were washed free of externally located bacteria and the internalised bacteria were allowed to incubate within the maturing phagosomal environment for $0,2,8$ or $24 \mathrm{~h}$. The bacteria were then recovered from infected macrophages by application of macrophage lysis solution and differential centrifugation at $400 \times \mathrm{g}(4 \mathrm{~min})$ then $4000 \times \mathrm{g}(4 \mathrm{~min})$. Bacteria were then stained with flow cytometry staining solution and the number of viable cells quantified using the ratio of bacteria signals to Bacteria Counting Kit bead signal, measured by flow cytometry. Plotted data represent the means of three independent experiments \pm 1 standard deviation. 


\subsubsection{Observations made during phagosomal maturation}

In the process of monitoring $P$. aeruginosa viability across a phagosome maturation time course, two observations were made with respect to (i) the number of viable wild type bacteria collected at $2 \mathrm{~h}$ maturation using a $\mathrm{MOI}$ of 50 compared to a $\mathrm{MOI}$ of 100 , and (ii) the phenotypic profile changes to $P$. aeruginosa during maturation as represented on the flow cytometry visual cytogram output.

As noted previously, wild type $P$. aeruginosa (MOI 100) left to mature within the phagosomal environment for an additional $2 \mathrm{~h}$ post infection showed a significant reduction in viability. This reduction in survival was even more pronounced at $8 \mathrm{~h}$. However, during the process of optimisation, an $\mathrm{MOI}$ of 50 was examined under identical infection and phagosomal maturation conditions, and found to give an entirely different result. At a $\mathrm{MOI}$ of 50 , the number of viable wild type bacteria significantly increased 14 -fold $(p<0.0001)$ at $2 \mathrm{~h}$ phagosomal maturation. By $8 \mathrm{~h}$ this number then dropped to below the number recorded at $0 \mathrm{~h}$ (Figure 4.11). We hypothesise that this effect resulted from differences in the $P$. aeruginosa population density and associated quorum sensing signals relative to the MOI 100 scenario. This observation was not pursued due to lack of relevance to the primary objectives of the project, however it does emphasise the profound difference that seemingly minor changes in an experimental parameter can have on the outcome of a study. 


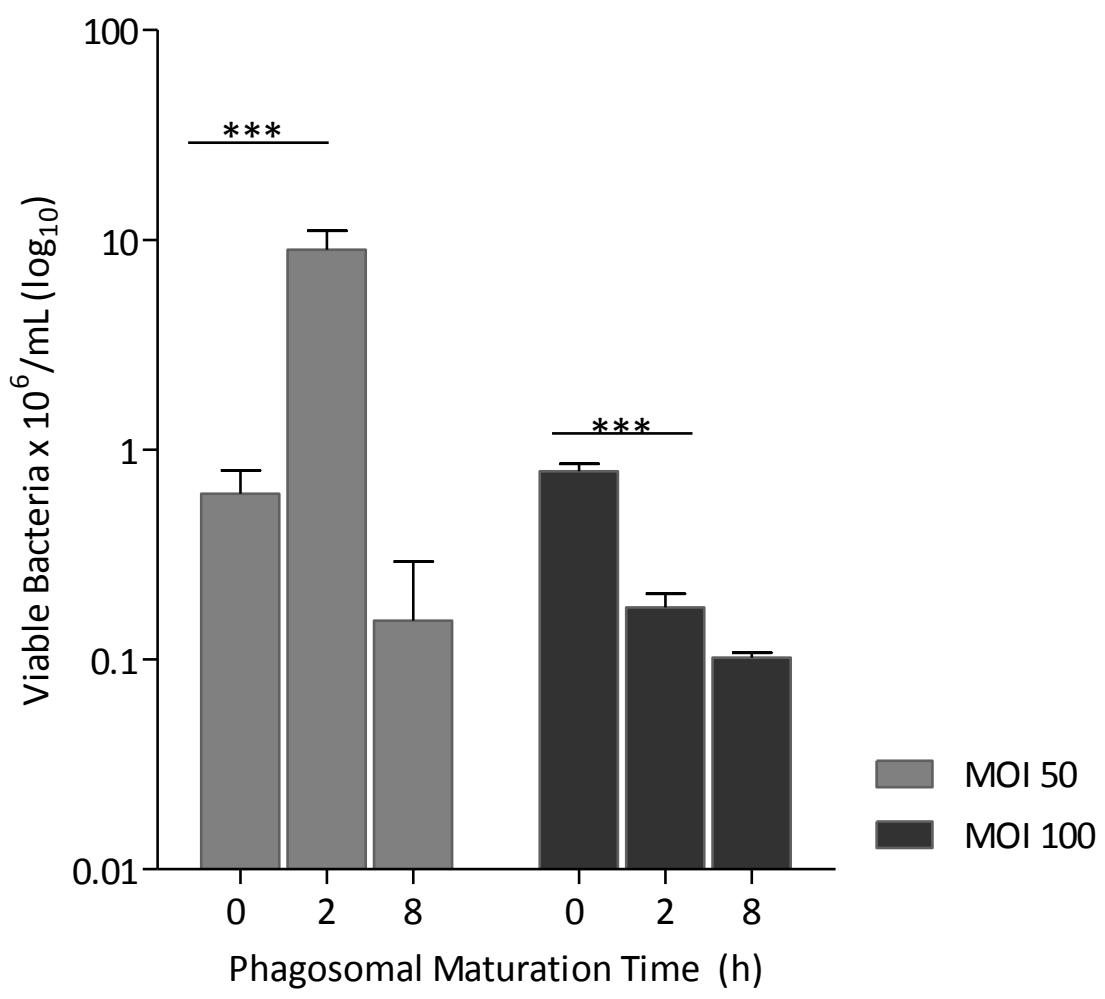

Figure 4.11. Viability of PAO1 during phagosomal maturation at MOIs of $\mathbf{5 0}$ and $\mathbf{1 0 0}$ RAW-264.7 macrophages were infected for $2 \mathrm{~h}$ with a $\mathrm{MOI}$ of 100 opsonised wild type PAO1 followed by a $100 \mu \mathrm{g} / \mathrm{mL}$ gentamycin treatment for $45 \mathrm{~min}$. The infected macrophages were washed free of externally located bacteria and the internalised bacteria were allowed to incubate within the maturing phagosomal environment for 0,2 or $8 \mathrm{~h}$. The bacteria were then recovered from infected macrophages by application of macrophage lysis solution and differential centrifugation at $400 \times \mathrm{g}(4 \mathrm{~min})$ then $4000 \mathrm{x}$ $g(4 \mathrm{~min})$. Bacteria were then stained with flow cytometry staining solution and the number of viable cells quantified using the ratio of bacteria signals to Bacteria Counting Kit bead signal, measured by flow cytometry. Plotted data represent the means of three independent experiments \pm 1 standard deviation.

As per the above maturation profile (Figure 4.10), an interesting observation regarding phenotypic profile changes in the flow cytometry graphical output was also noted for the MOI 50 infection conditions. In short, at $2 \mathrm{~h}$ post phagosomal maturation, the physical location of the wild type PAO1 population on a cytogram of $\mathrm{SYTO}^{\circledR} \mathrm{BC}$ vs. PI was consistently seen to shift from the $\mathrm{SYTO}^{\circledR} \mathrm{BC}+\mathrm{PI}$ - quadrant to the $\mathrm{SYTO}^{\circledR} \mathrm{BC}+\mathrm{PI}+$ quadrant (Figure 4.9). Although this population appears to acquire the dead cell stain at $2 \mathrm{~h}$ post maturation, it was previously determined by cell sorting (Figure 4.2), that the double positive bacterial populations are viable and therefore counted towards total survival. It is unknown why this shift occurred and was possibly related to a staining artefact, biological event or another unknown factor. As this observation was only seen with a $\mathrm{MOI}$ of 50 , it was not deemed relevant and infection studies proceeded with a MOI of 100 as detailed earlier. 

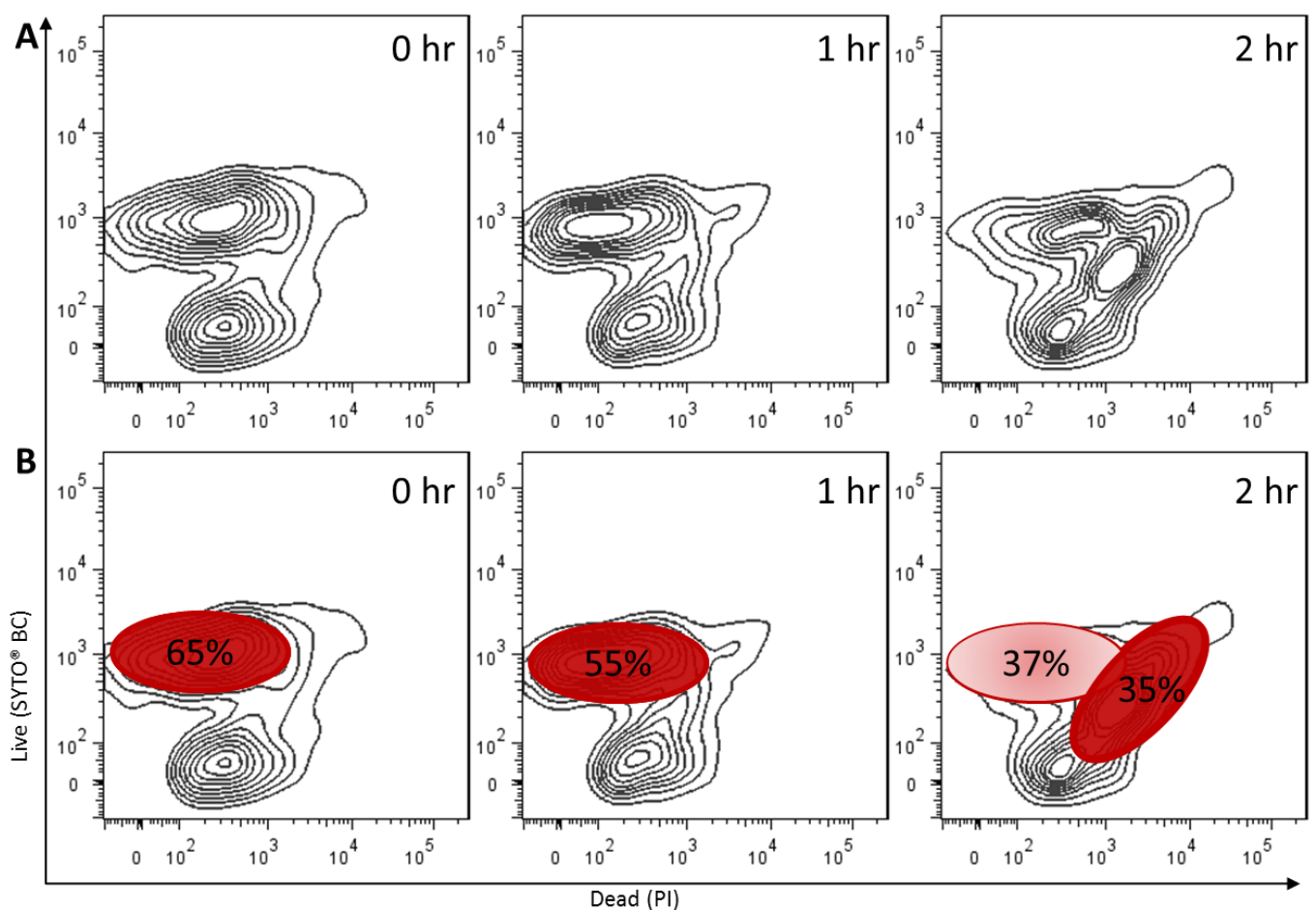

Figure 4.12. Phenotypic changes of PAO1 viabilty (MOI 50) as represented on cytogram

(A) RAW-264.7 macrophages were infected for $2 \mathrm{~h}$ with opsonised wild type PAO1 (MOI 50) followed by a $100 \mu \mathrm{g} / \mathrm{mL}$ gentamycin treatment for $45 \mathrm{~min}$. The infected macrophages were washed free of externally located bacteria and the internalised bacteria were allowed to incubate within the maturing phagosomal environment for 0,1 or $2 \mathrm{~h}$. The bacteria were then recovered from infected macrophages by application of macrophage lysis solution and differential centrifugation at $400 \times \mathrm{g}$ (4 min) then $4000 \mathrm{x}$ $g$ (4 min). The bacteria were stained with flow cytometry staining solution and the number of viable cells quantified using the ratio of bacteria signals to Bacteria Counting Kit bead signal, measured by flow cytometry. This observation appeared in as many as 16 replicates. This figure presents a representative of one replicate. (B) Total live cell events $\left(\mathrm{SYTO}^{\circledR} \mathrm{BC}+\right.$ ) as a percentage of total plotted events at 0,1 , and $2 \mathrm{~h}$ phagosomal maturation were overlaid on the respective cytograms of wild type PAO1 (MOI 50).

In summary, by sequentially testing individual parameters, an in vivo cell culture infection model to evaluate $P$. aeruginosa response to murine macrophages was developed. It was determined that for maximal RNA isolation, RAW-264.7 macrophages were best able to take up opsonised-P. aeruginosa in the absence of IFN$\gamma$ and that an initial MOI of 100 generated the greatest yield of total prokaryotic RNA. Additionally, harvesting the bacteria immediately after an infection of $2 \mathrm{~h}$, plus 45 min gentamycin treatment (i.e. $\mathrm{T}=0$ ), resulted in the greatest harvesting of viable bacteria, as opposed to situations where the phagosomal environment was allowed to mature post infection. Although some surprising apparent changes in cellular responses or phenotype were observed during phagosomal maturation at a $\mathrm{MOI}$ of 50 , these phenomena were not pursued further in this study. Instead, having finalised the 
experimental parameters, the next step was to apply the optimised in vivo cell culture model to (i) characterise the relative survival of NQOR-deleted or over-expressing PAO1 strains (and also clinical isolates) post phagocytosis, and (ii) assess the global transcriptional response of wild type PAO1 post internalisation by RAW-264.7 macrophages.

\subsection{In vivo cell culture infection screening of $P$. aeruginosa strains}

\subsubsection{Clinical isolates}

Several New Zealand $P$. aeruginosa clinical isolates were evaluated for survival post RAW-264.7 macrophage exposure (Figure 4.13). The five clinical isolates tested came from a total of four patients and represented non-mucoid strains identified in numerous biological samples from blood to urine to tracheal aspirates (Chapter 2, Table 2.2). All five clinical isolates were tested in vitro for sensitivity to $\mathrm{H}_{2} \mathrm{O}_{2}$ compared to PAO1 as described in Section 2.10, but this indicated no differential sensitivities (data not shown). Consistent with the in vitro characterisation, macrophage engulfment of clinical isolates ARL1079 (tracheal aspirate), ARL1205 (thigh), and ARL1236 (blood) demonstrated no substantial difference in survival when compared to PAO1. However ARL0011323, isolated from cystic fibrosis sputum, was able to withstand macrophage exposure $>4$ fold better than wild type PAO1 $(p<0.01)$. ARL1024, a sample from a catheter, was also significantly more sensitive to RAW-264.7 macrophage exposure $(p<0.01)$. 


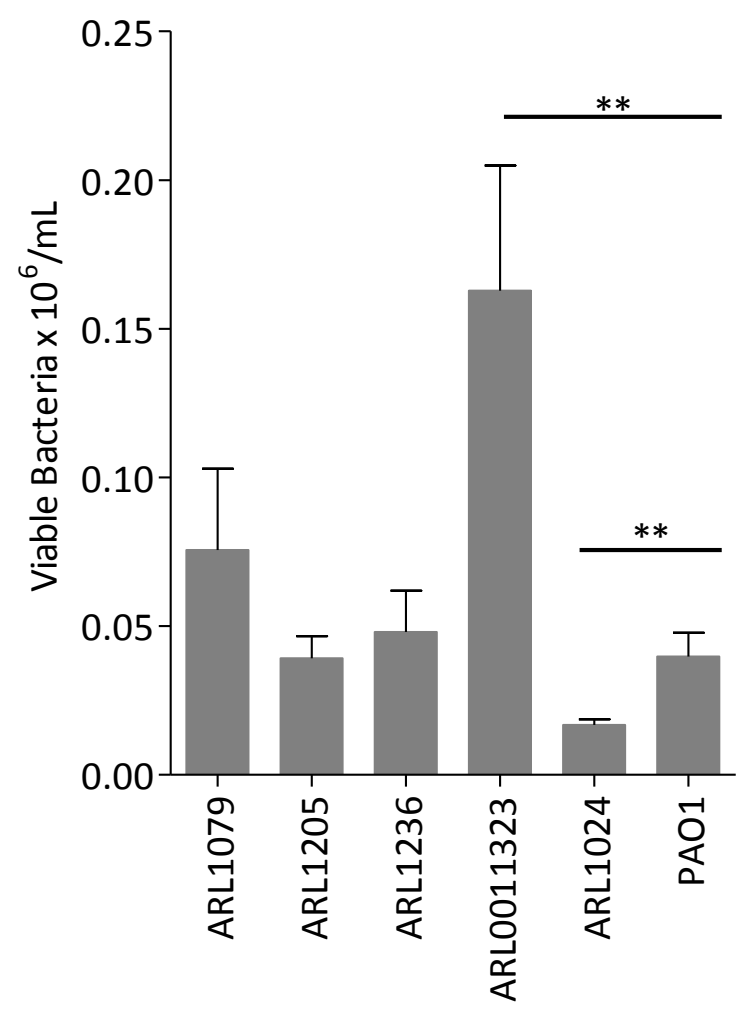

Figure 4.13. Viable $P$. aeruginosa clinical strains recovered from macrophage infection

RAW-264.7 macrophages were infected for $2 \mathrm{~h}$ with a MOI of 100 opsonised clinical $P$. aeruginosa isolates, followed by a $100 \mu \mathrm{g} / \mathrm{mL}$ gentamycin treatment for $45 \mathrm{~min}$. The infected macrophages were then washed free of externally located bacteria. The internalised bacteria were recovered from infected macrophages by application of macrophage lysis solution and differential centrifugation at 400x $g$ (4 $\mathrm{min}$ ) then $4000 \mathrm{xg}(4 \mathrm{~min})$. The bacteria were then stained with flow cytometry staining solution and the number of viable cells quantified using the ratio of bacteria signals to Bacteria Counting Kit bead signal measured by flow cytometry. Plotted data represent the means of three independent experiments \pm 1 standard deviation. Significance is reported where $p<0.01\left({ }^{* *}\right)$.

\subsubsection{Mutant and over-expression strains}

The macrophage model was also used to expand upon the preliminary NQOR enzyme characterisation described in Chapter 3. Although the single mutant $\Delta 1204$ did not previously exhibit heightened sensitivity to $\mathrm{H}_{2} \mathrm{O}_{2}$ challenge in vitro, this mutant did appear to be more sensitive to macrophage infection (Figure $4.14 \mathrm{~A}$ ). When the $\Delta 1204$ mutant was compared to the $\Delta k a t A$ mutant there was no significant difference between either strain; whereas both of these strains were significantly more sensitive than wild type PAO1 ( $p<0.05$ for both the $\Delta 1204$ and $\Delta k a t A$ mutants).

As demonstrated in the number of viable ARL1323 and $\Delta 1204 P$. aeruginosa recovered from infection (Figure 4.13 and Figure 4.14A respectively), compared to wild 
type PAO1, the in vivo cell culture model was able to distinguish differential sensitivities between (i) clinical isolates and (ii) NQOR-deleted strains that had not previously been identified. In contrast, over-expression strains (in a katA-null background) recovered from macrophages exhibited similar patterns of survival as observed in Chapter 3 (Section 3.3.2.2) when over-expression strains were challenged in vitro with $\mathrm{H}_{2} \mathrm{O}_{2}$ (Figure 4.14B). The over expression of PA2580, PA0949, or KatA (PA4236) enabled PAO1 to withstand engulfment by murine macrophages $3.8-5.5$ fold better than wild type PAO1 (Figure 4.14B).
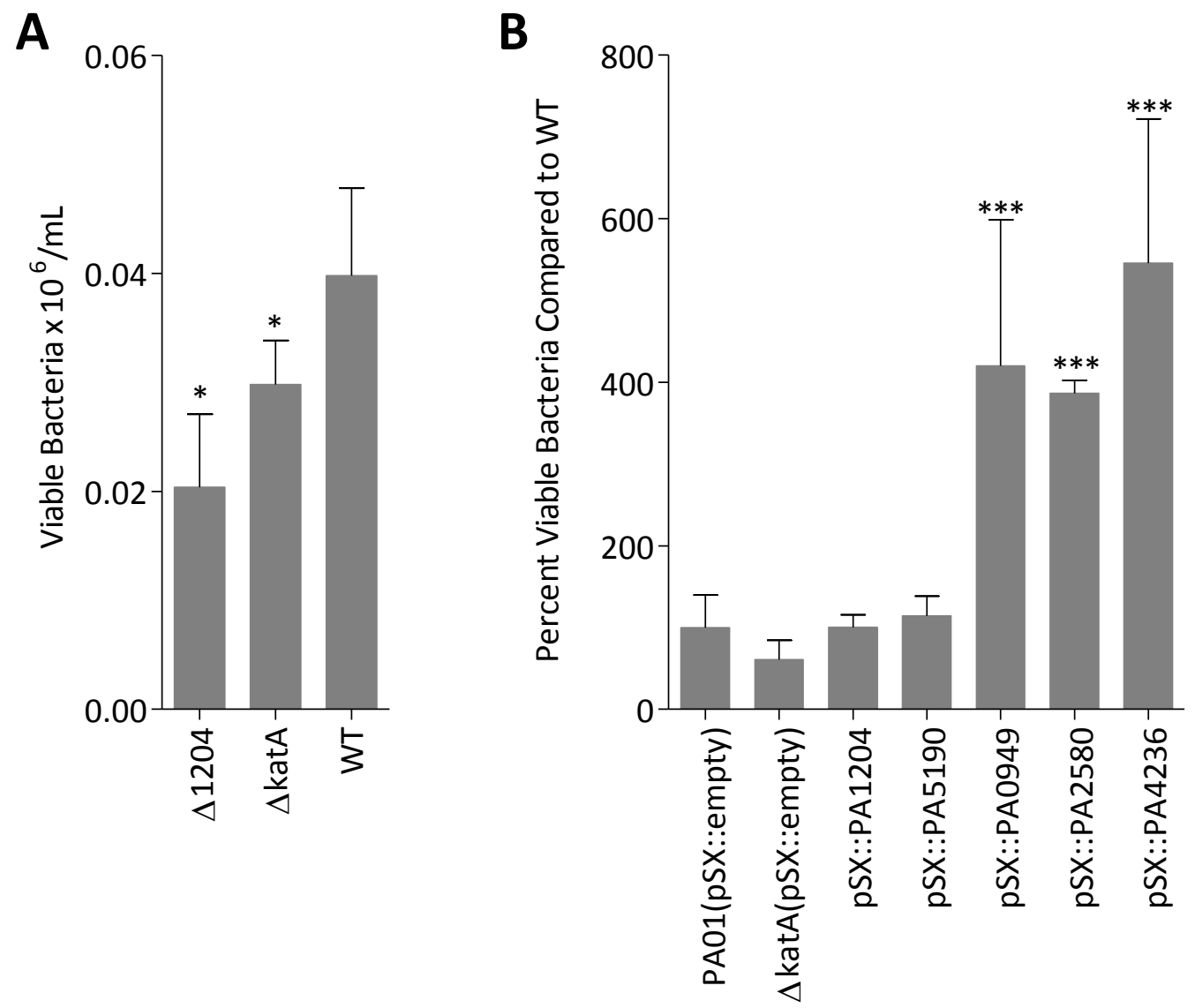

\section{Figure 4.14. Viable $P$. aeruginosa NQOR-deletion and NQOR over-expression strains recovered from macrophage infection}

RAW-264.7 macrophages were infected for $2 \mathrm{~h}$ with a MOI of 100 opsonised $(\mathrm{A})$ NQOR gene-deleted PAO1 strains, or (B) NQOR over-expressing PAO1 strains (including empty pSX vector controls in wild type PAO1 and PAO1, $\triangle$ katA where over-expression was induced and maintained by amending the growth media with $0.25 \mathrm{mM}$ IPTG and $10 \mu \mathrm{g} / \mathrm{mL}$ gentamycin) followed by a $100 \mu \mathrm{g} / \mathrm{mL}$ gentamycin treatment for $45 \mathrm{~min}$. The internalised bacteria were recovered from infected macrophages by application of macrophage lysis solution and differential centrifugation at 400x $g$ (4 min) then 4000x $g$ ( 4 $\mathrm{min})$. The bacteria were then stained with flow cytometry staining solution and the number of viable cells quantified using the ratio of bacteria signals to Bacteria Counting Kit bead signal measured by flow cytometry. Plotted data represent the means of three independent experiments \pm 1 standard deviation. Significance is reported where $p<0.05\left(^{*}\right)$ and $p<0.001\left({ }^{* *}\right)$ are compared to WT (A) and PAO1 (B) controls. Data presented in (B) is indicative of a ratio of viable bacteria to the PAO1 control because of day-to-day variations; between same-day replicates variation was low, and general trends were highly reproducible. 
The NQOR over-expression strains recovered from macrophage infection were additionally phenotypically represented on a cytogram to demonstrate changes in viability of selected strains between 0 and $2 \mathrm{~h}$ of phagosomal maturation (Figure 4.15A). It was noted that the $2 \mathrm{~h}$ phagosome-matured viable populations responded to macrophage infection in a manner consistent with their response to in vitro $1.5 \mathrm{mM}$ $\mathrm{H}_{2} \mathrm{O}_{2}$ challenge as previously described in Chapter 3 (section 3.3.2.2; reproduced in Figure 4.15B). The pSX::PA1204 over-expression strain, like $\Delta$ katA (with pSX::empty) showed sensitivity to macrophage infection after $2 \mathrm{~h}$ phagosomal maturation; whereas the pSX::katA and pSX::PA2580 strains, like PAO1 (with pSX::empty), were able to survived engulfment at the same time point. 

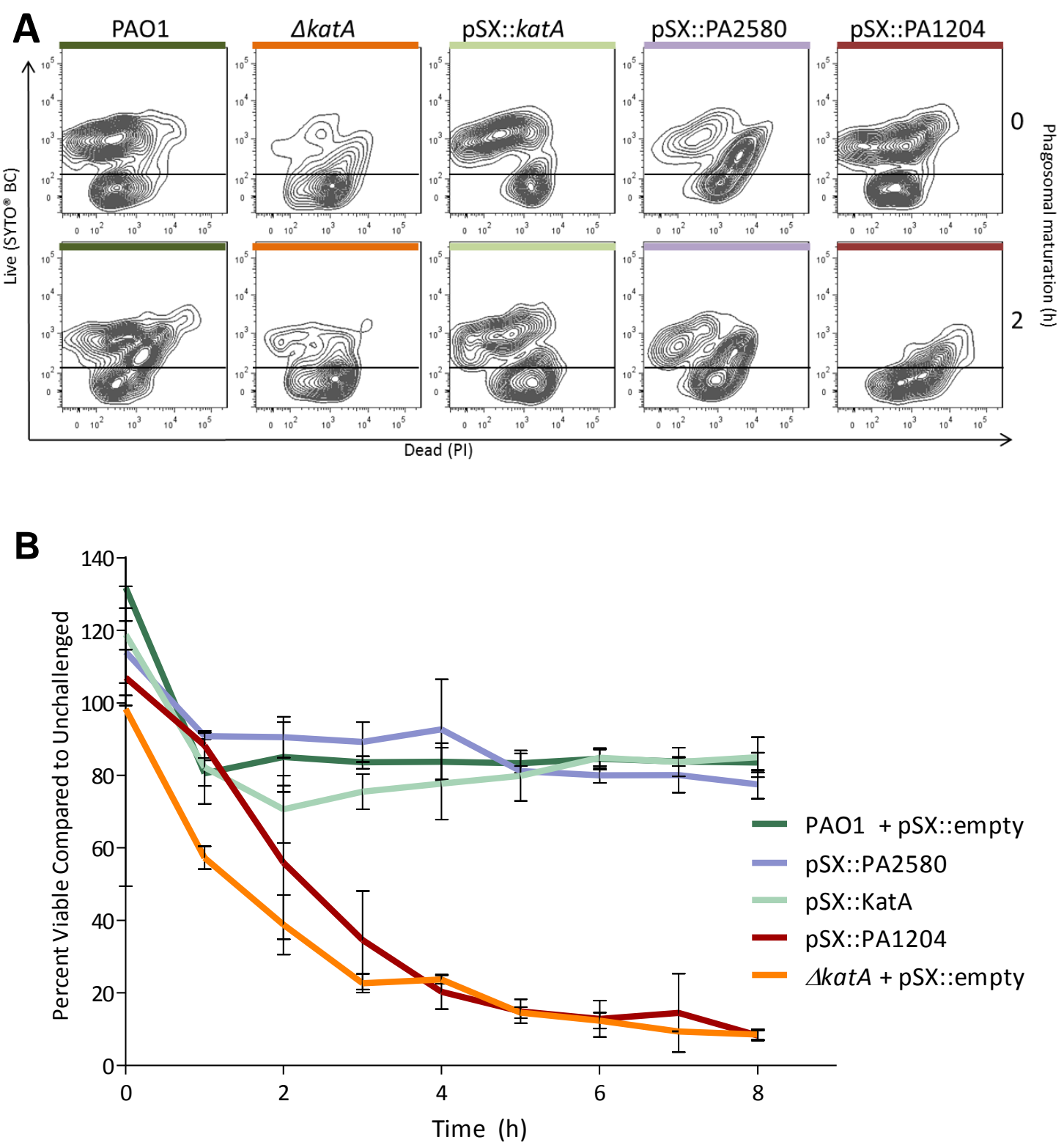

Figure 4.15. Comparison of survival patterns of NQOR over-expression strains following macrophage infection and in vitro $\mathrm{H}_{2} \mathrm{O}_{2}$ challenge

(A) $3 \mathrm{~mL}$ cultures of NQOR over-expression strains were grown overnight in LB supplemented with 0.25 $\mathrm{mM}$ IPTG and $10 \mu \mathrm{g} / \mathrm{mL}$ gentamycin to induce NQOR over-expression. RAW-264.7 macrophages were then infected for $2 \mathrm{~h}$ with PBS-washed opsonised NQOR over-expression strains (MOI 100) in media supplemented with $0.25 \mathrm{mM}$ IPTG. Infection was followed by $100 \mu \mathrm{g} / \mathrm{mL}$ gentamycin treatment for 45 $\min$. The internalised bacteria were then recovered from infected macrophages by application of macrophage lysis solution and differential centrifugation at 400x $g$ (4 min) then 4000x $g$ (4 min). The bacteria were stained with flow cytometry staining solution and the viable bacterial populations were visualised on a cytogram of SYTO ${ }^{\circledR} \mathrm{BC}$ vs. PI. This was repeated in three independent experiments with consistent results. Panel $A$ is a representative cytogram of one replicate. (B) Over-expression strains were inoculated to an initial $O_{660}$ of 0.1 in LB amended with $0.25 \mathrm{mM} \mathrm{IPTG}, 10 \mu \mathrm{g} / \mathrm{mL}$ gentamycin and \pm $1.5 \mathrm{mM} \mathrm{H}_{2} \mathrm{O}_{2}$. The $\mathrm{OD}_{660}$ of challenged and unchallenged cultures was measured hourly for $9 \mathrm{~h}$. The plotted data are means of 3 independent experiments, each performed in duplicate, and error bars are \pm 1 standard deviation. 


\subsection{Modification of infection conditions for RNA isolation}

For gene expression profiling of $P$. aeruginosa by microarray, three distinct samples of RNA were prepared - from: (i) Internal PAO1 - bacteria engulfed by macrophages, (ii) External PAO1 - bacteria exposed but not internalised by macrophages, and (iii) Control PAO1 - bacteria incubated in CMEM free of macrophages for the same duration as (i) and (ii). A total of $30 \mu \mathrm{g}$ of prokaryotic RNA was required for each of the three experimental conditions ( $10 \mu \mathrm{g}$ for each of three replicates) in the microarray application. To maximise the number of bacteria harvested for RNA isolation to achieve $30 \mu \mathrm{g}$, the macrophage monolayer in all experimental conditions (except for Control PAO1) was prepared by seeding 6 -well plates with $2.5 \times 10^{5}$ cells in a volume of $3 \mathrm{~mL} \mathrm{CMEM}$. The MOI of 100 was then maintained by adjusting the concentration of PAO1 to proportionately match the increased number of RAW-264.7 macrophages in culture.

To isolate RNA from Internal PAO1, RAW-264.7 macrophages were infected for $2 \mathrm{~h}$ (including 45 min gentamycin treatment) as described previously. The postinfection washing steps were completed in less than 2 min for each sample (as described in section 4.4.2) before covering the washed monolayer in $1 \mathrm{~mL} \mathrm{TRIzol}{ }^{\circledast} / \mathrm{cm}^{2}$ for $5 \mathrm{~min}$ at room temperature to preserve both eukaryotic and prokaryotic RNA. This was to minimize artefactual changes in RNA expression due to the collection process. The TRIzol ${ }^{\circledR}$-lysed mixtures for each of the 24 wells collected were then combined in an RNase-free $15 \mathrm{~mL}$ conical tube and stored at $-80{ }^{\circ} \mathrm{C}$ overnight prior to RNA purification.

To isolate RNA from External PAO1, macrophages were infected as above, but instead of discarding each of the four PBS washes post infection, the supernatant from the infection and each wash was combined and pelleted by centrifugation at 4,000x $g$ for $5 \mathrm{~min}$ at $4{ }^{\circ} \mathrm{C}$ in a $15 \mathrm{~mL}$ RNase-free conical tube. The pellet was initially resuspended in $1 \mathrm{~mL} \mathrm{TRIzol}{ }^{\circledR}$, then further diluted to a final volume of $3 \mathrm{~mL} \mathrm{TRIzol}{ }^{\circledR}$. This TRIzol ${ }^{\circledR}$-lysate was then stored overnight at $-80^{\circ} \mathrm{C}$.

RNA from Control PAO1 (incubated in CMEM at the same concentration as that administered to achieve a $\mathrm{MOI}$ of 100 when macrophages were present) was prepared by incubating the washed and opsonised PAO1 (as above) in CMEM in 6-well culture plates free of macrophages. These were incubated for $2.75 \mathrm{~h}$ at $37{ }^{\circ} \mathrm{C}, 5 \% \mathrm{CO}_{2}$, such that they were maintained under cell culture conditions for the same duration as the 
Internal PAO1 and External PAO1 bacteria. Control PAO1 were then harvested and treated per the External PAO1 samples, above.

\subsubsection{RNA quality control}

Purified RNA from all three sample types was assessed for concentration, purity and integrity by the Agilent 2100 Bioanalyzer. Concentrations were calculated from the total area of prokaryotic $16 \mathrm{~S}$ and $23 \mathrm{~S}$ ribosomal subunit peaks as a percentage of total surface area (x-axis) of each respective electrophoretic trace. The integrity of each RNA sample was computationally predicted by the Bioanalyzer software using the RNA Integrity Number (RIN) scoring tool. RIN assessment uses the electrophoretic trace of the RNA sample to assign a value of $1-10$, where level 10 represents completely intact RNA. The RIN algorithm is independent of sample concentration, instrument and user bias (229). All RNA samples submitted for this microarray experiment possessed RIN scores $\geq 8.5$. RNA sample preparation purity was visually assessed using the electrophoretic trace (Figure 4.16). Enrichment of Internal PAO1 was found to remove $>90 \%$ of eukaryotic RNA contamination (Figure 4.16AB). The External PAO1 RNA was found to contain minor traces of eukaryotic RNA (Figure 4.16C). Control PAO1 RNA was almost $100 \%$ pure (Figure $4.16 \mathrm{D}$ ).

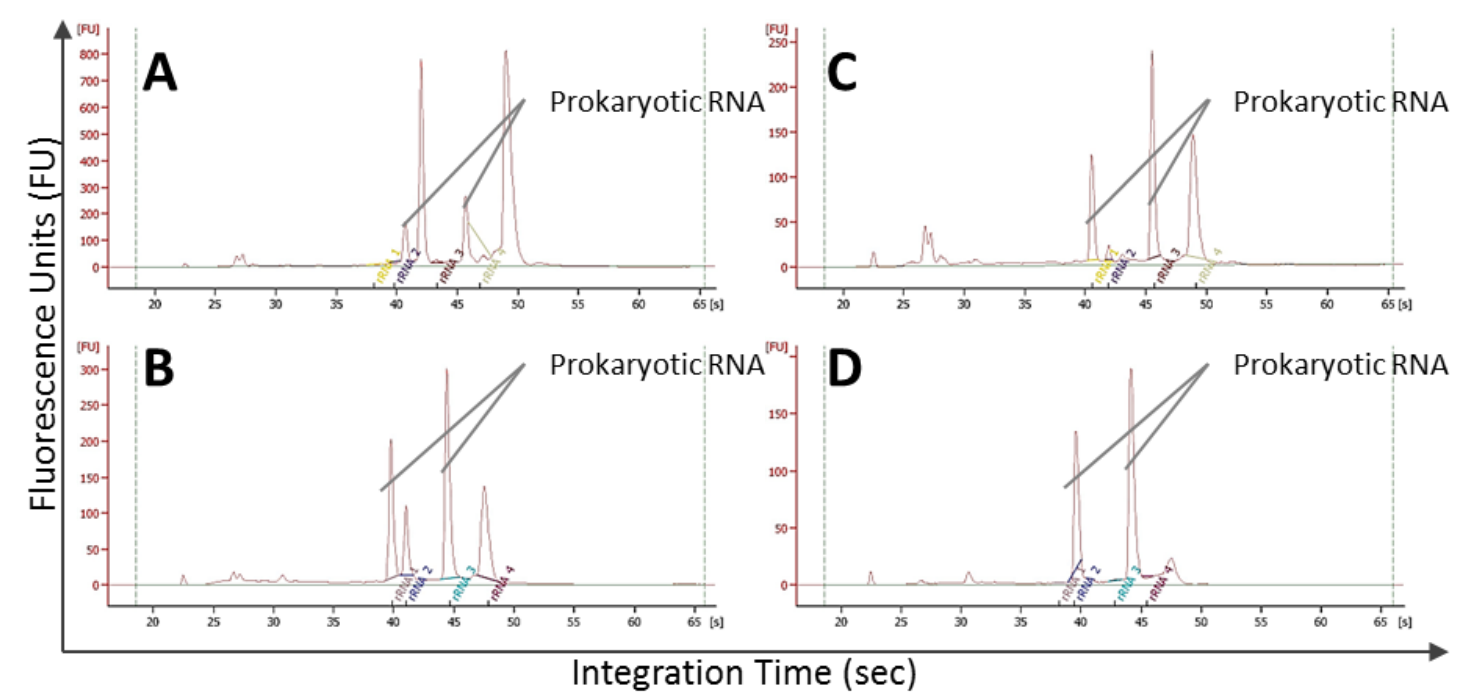

Figure 4.16. RNA quality control

Purified Internal PAO1, External PAO1, and Control PAO1 RNA samples in $1.5 \mu \mathrm{L}$ RNase-free water assessed for concentration, purity and integrity by the Agilent 2100 Bioanalyzer. Electrophoretic traces of each RNA samples indicated quantitative peaks for eukaryotic $18 \mathrm{~S}$ and $28 \mathrm{~S}$ and/or prokaryotic $16 \mathrm{~S}$ and 235 ribosomal subunits by calculating the area under the curve. (A) Internal PAO1 pre-enrichment, (B) Internal PAO1 post-enrichment, (C) External PAO1, (D) Control PAO1. 


\subsection{Transcriptional profile of internalised $P$. aeruginosa}

Purified RNA samples were submitted to the Centre for Genomics, Proteomics and Metabolomics (CGPM) at the University of Auckland for processing and scanning. Raw expression data of triplicate samples, as performed by Dr Donia Macartney-Coxson of ESR, were averaged and expression values normalised by RMA (robust multi-array chip averaging) as instigated in the Affy and Oligo packages in the open source Bioconductor analysis software. Expression fold change for genes in Internal PAO1 and External PAO1 samples were calculated in relation to Control PAO1. Tables of all genes meeting quality control conditions and the statistical cut of $p<0.05$ are listed in Appendix A2 and summarised in Table 4.3. Additionally, each gene, taking the expression fold change into consideration, was assigned a B-score. The B-score is a statistical measurement of the log odds that a gene is differentially expressed where a score of 0 is equal to a $50 \%$ probability of differential expression and 3 is $~ 95 \%$ probability $(230,231)$.

Using generic cut off measures of $>2$ fold expression change (FC) there appeared to be some overlap in genes regulated by $P$. aeruginosa both in Internal PAO1 and External PAO1 samples relative to the unchallenged Control PAO1. However, the vast majority of gene groups were unique to each respective sample with the exception of the down-regulated genes of External PAO1, in which 78 (30.5\%) genes overlap with the 255 Internal PAO1 down-regulated genes (Figure 4.13).

Table 4.3. Gene expression compared to Control PAO1 $(p<0.05)$

\begin{tabular}{lccc}
\hline Sample & $\begin{array}{c}\text { Total genes with } \\
\mathrm{p}<0.05\end{array}$ & $>2 \mathrm{FC}^{\mathrm{a}}$ & $>3 \mathrm{FC}^{\mathrm{a}}$ \\
\hline Internal PAO1 & 1856 & 151 & 30 \\
$\quad$ Up-regulated & 1691 & 255 & 79 \\
$\quad$ Down-regulated $^{b}$ & & & \\
External PAO1 $^{\text {Up-regulated }}$ & 1274 & 105 & 14 \\
Down-regulated $^{b}$ & 1667 & 87 & 11 \\
\hline
\end{tabular}

\footnotetext{
${ }^{a}$ Fold change expression compared to Control PAO1.

b "Up-regulated" and "down-regulated" refer to a positive or negative change in expression fold change respectively.
} 


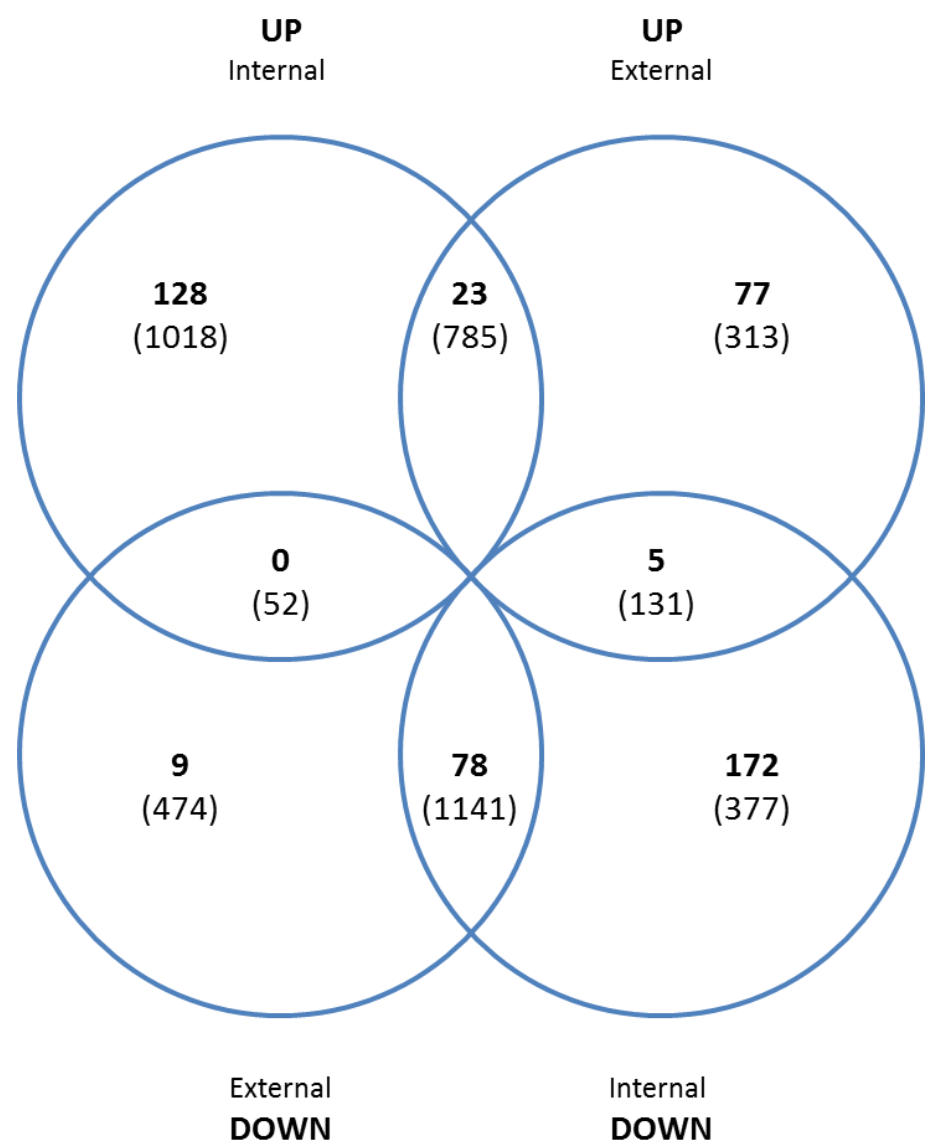

Figure 4.17. Distribution of activated and repressed genes between Internal PAO1 and External PAO1 in relation to Control PAO1

Bold values indicate $>2 \mathrm{FC}$ whereas bracketed numbers indicate total genes up- or down- regulated with $\mathrm{p}<0.05$.

Top tables of expression data were sorted by functional characterisation of the gene groups as annotated in the Pseudomonas Genome Database to identify possible trends in regulation. "Hypothetical, unclassified, unknown" or "Putative" gene annotations made up the bulk of each the four data sets (37.75\%, Internal PAO1-upregulated; 46.52\%, Internal PAO1-down-regulated; 14.05\%, External PAO1-upregulated; 54.46\%, External PAO1-down-regulated) (Appendix A2). For the genes of known function, in response to challenge by macrophages the internalised $P$. aeruginosa appear to be up-regulating transcriptional regulators, two-component regulatory systems, secreted factors, chemotactic proteins, and genes related to motility and attachment - all of which are consistent with a defensive response to a threat (Figure 4.14, complete set Appendix A3). Interestingly, 128 intergenic regions were up-regulated as well. Intergenic regions have largely been thought to contain small non-coding RNAs; however, more recent studies suggest that intergenic regions 
may possess functional small-length coding regions not normally annotated in gene prediction programs (232-234). Microbial genomes have now been found to contain functional elements like insertion sequences (transposable elements) contained within the intergenic regions $(235,236)$.

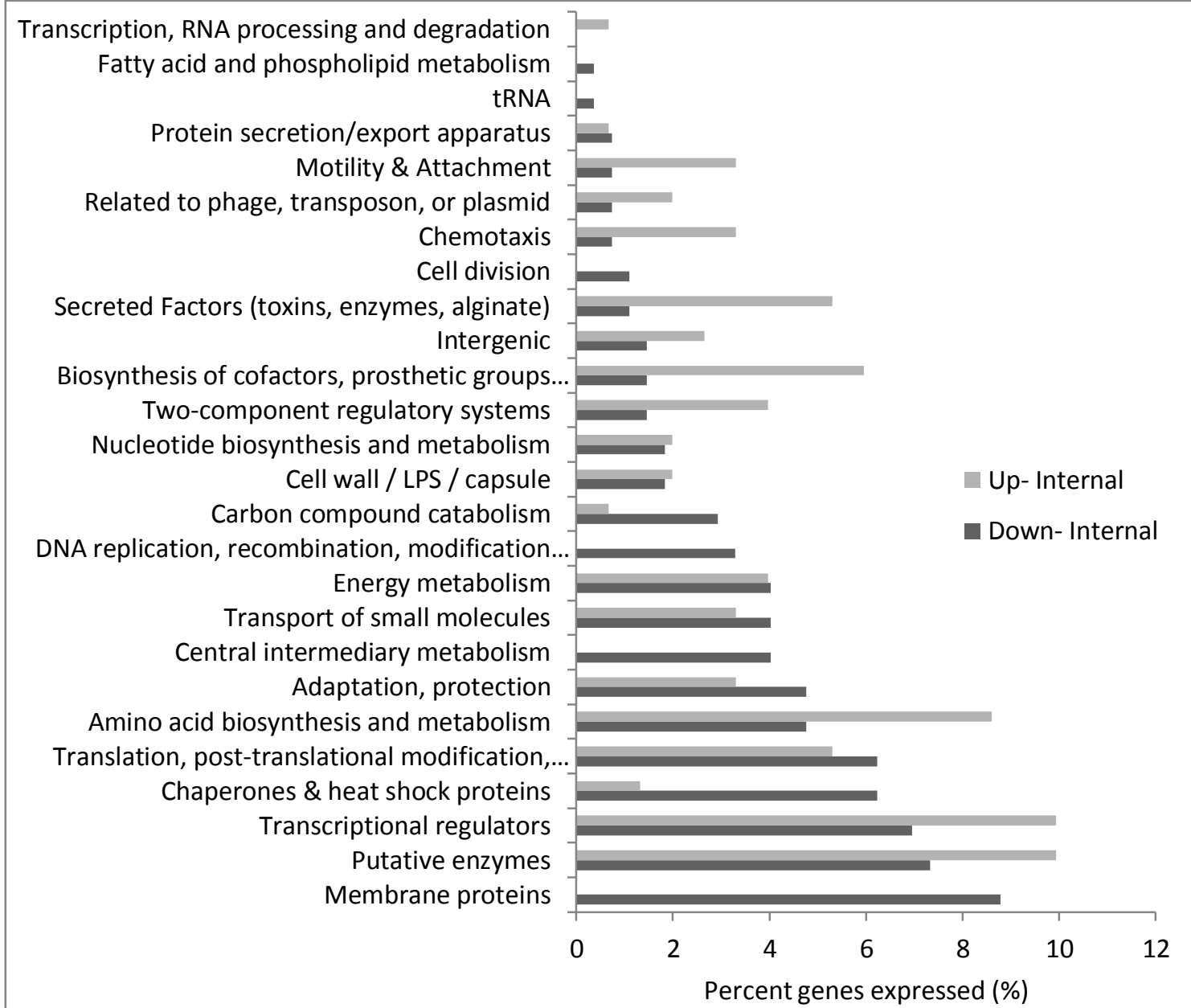

Figure 4.18. Functional classification of Internal PAO1 genes

Internal PAO1 genes with >2 FC expression are listed by functional annotation as a percentage of annotation occurrence within the data set. "Hypothetical, unclassified, unknown" genes were excluded from this analysis. 


\subsubsection{Interpretation and analysis of functional gene groups}

Of the wealth of information provided by the microarray, expression data sets were categorised according to two different strategies. Firstly, genes were grouped by functional relationship using the Database for Annotation, Visualization and Integrated Discovery (DAVID) v6.7. DAVID is a web-based tool that provides functional annotation for large lists of genes and enriches for biological themes and function-related gene groups $(156,237)$. DAVID uses gene ontology $(\mathrm{GO})$-based functional similarity and assigns genes to "functional group" based either on the number of genes within a category or by the EASE-score. The DAVID EASE score estimates the likelihood that a functional association is due to random chance (the higher the score the more functionally enriched (i.e. the more likely the biological significance of the gene group; $(156,237))$. After assigning genes to a category and ranking enrichment by EASE score, individual genes within that group are further assigned a Benjamini score. Benjamini scores are adjusted p-values used to indicate "false discovery in the case of weak induction" meaning that, like the p-value, the lower the Benjamini score the more likely a "true" expression signal (238). The statistical scores (EASE, p-value, and Benjamini), in combination, are used to assess the likelihood each gene within a "cluster" is involved in a similar biological mechanism.

A secondary approach to classifying the $P$. aeruginosa response to macrophages was to match expression data with corresponding operons listed in the Database of prOkaryotic OpeRons (DOOR) v2.0 (239). In bacteria, genes are generally organised into operons or co-regulated clusters. Genes within the same operon typically share only small intergenic regions and are co-expressed. DOOR uses known operon characteristics to computationally predict operons consisting of conserved hypothetical genes. 


\subsubsection{Functional Clustering using DAVID}

Because of the numerous genes in each data set, computationally predicted gene groups which share functional relationships could be diluted. Essentially, the more that genes which share only a small degree of functional congruence are "forced" into a functional relationship, the more generic a functional annotation becomes. To avoid this scenario, functional clustering was performed using only genes that possessed $\geq 3$ FC expression over Control PAO1. The narrowed data set clustering was performed under the highest stringency settings in DAVID.

Using the Internal PAO1-up-regulated data set as an example, of the $>5,500$ open reading frames (ORF) and intergenic regions of internalised $P$. aeruginosa screened, 1856 unique sequences had an Benajmini scores $\leq 0.05$ (Benjamini method to control false discovery rate; (238)). Of those, 30 ORFs were up-regulated $>3 \mathrm{FC}$ and assessed for functional clustering using DAVID. Only 19 of the 30 ORFs were identified as belonging to an enriched functional gene group cluster. This is in part due to the fact that over $27.8 \%$ of the genes present in the Internal PAO1-up-regulated Top Table are currently not annotated in the Pseudomonas Genome Database and are otherwise characterised as being "hypothetical" (Appendix Table A2.1).

The five functional clusters detected in the Internal PAO1-up-regulated data set represent DNA repair proteins, heat shock / chaperone proteins, RNA polymerase sigma factors, the LexA repressor, and alginate regulation (Table 4.4). The two downregulated functional clusters include pyocin-S2, cellular detoxification processes, and fimbrial assembly proteins (Table 4.5). However, these down-regulated groups possessed low enrichment (EASE) scores $<0.1$ suggesting that the functional association between genes within those clusters were more likely to have been due to chance. 
Table 4.4. Internal PAO1 up-regulated functional clustering

\begin{tabular}{|c|c|c|c|c|}
\hline & ID & Gene Name & & \\
\hline \multirow[t]{4}{*}{1} & \multicolumn{2}{|c|}{ DNA Repair/Response to DNA Damage Stimulus } & \multicolumn{2}{|c|}{ Enrichment Score: 1.69} \\
\hline & PA3007_lexA_at & LexA repressor & P_Value & Benjamini \\
\hline & PA3617_recA_at & Protein RecA & $2.20 \mathrm{E}-03$ & $2.10 \mathrm{E}-02$ \\
\hline & PA0404_i_at & Putative Holliday junction resolvase & & \\
\hline \multirow[t]{6}{*}{2} & \multicolumn{2}{|c|}{ Heat shock/Chaperone/Protein folding } & \multicolumn{2}{|c|}{ Enrichment Score: 1.03} \\
\hline & PA4386_groES_at & 10 kDa chaperone & P_Value & Benjamini \\
\hline & PA4760_dnaJ_at & Chaperone protein DnaJ & $6.20 \mathrm{E}-05$ & 7.40E-03 \\
\hline & PA4761_dnaK_at & Chaperone protein DnaK & & \\
\hline & PA1596_htpG_at & Chaperone protein HtpG & & \\
\hline & PA4762_grpE_at & Heat shock protein GrpE & & \\
\hline \multirow[t]{8}{*}{3} & \multicolumn{2}{|c|}{ Transcription Regulation } & \multicolumn{2}{|c|}{ Enrichment Score: 0.46} \\
\hline & PA3161_himD_at & Integration host factor subunit beta & P_Value & Benjamini \\
\hline & PA3007_lexA_at & LexA repressor & $8.40 \mathrm{E}-02$ & 4.70E-01 \\
\hline & PA3006_at & transcriptional regulator PsrA & & \\
\hline & PA3815_at & Iron-sulfur cluster assembly transcription factor & & \\
\hline & PA3617_recA_at & Protein RecA & & \\
\hline & PA0762_algU_at & RNA polymerase sigma-H factor & & \\
\hline & PA0376_rpoH_at & RNA polymerase sigma-32 factor & & \\
\hline \multirow[t]{7}{*}{4} & Metal Ion Binding & & \multicolumn{2}{|c|}{ Enrichment Score: 0.43} \\
\hline & PA4760_dnaJ_at & Chaperone protein DnaJ & P_Value & Benjamini \\
\hline & PA1847_at & $\mathrm{Fe} / \mathrm{S}$ biogenesis protein NfuA & $6.40 \mathrm{E}-01$ & $9.70 \mathrm{E}-01$ \\
\hline & PA0665_at & Iron-sulfur cluster insertion protein ErpA & & \\
\hline & PA3524_gloA1_at & Lactoylglutathione lyase & & \\
\hline & PA3815_at & Iron-sulfur cluster assembly transcription factor & & \\
\hline & PA0376_rpoH_at & RNA polymerase sigma-32 factor & & \\
\hline \multirow[t]{4}{*}{5} & Cell Membrane & & \multicolumn{2}{|c|}{ Enrichment Score: 0.1} \\
\hline & PA3665_at & Putative threonine efflux protein & P_Value & Benjamini \\
\hline & PA0763_mucA_at & Sigma factor AlgU negative regulatory protein & $6.40 \mathrm{E}-01$ & $9.70 \mathrm{E}-01$ \\
\hline & PA2604_at & Conserved hypothetical protein & & \\
\hline
\end{tabular}

Table 4.5. Internal PAO1 down-regulated functional clustering.

\begin{tabular}{|c|c|c|c|c|}
\hline & ID & Gene Name & & \\
\hline \multirow[t]{5}{*}{1} & Metal Ion Binding & & & \\
\hline & PA1155_nrdB_at & Ribonucleotide reductase, beta subunit & \multicolumn{2}{|c|}{ Enrichment Score: 0.1} \\
\hline & PA3520_at & Hypothetical protein & P_Value & Benjamini \\
\hline & PA1150_pys2_at & Pyocin-S2 & 7.70E-01 & $1.00 \mathrm{E}+00$ \\
\hline & PA1317_cyoA_at & Ubiquinol oxidase subunit 2 & & \\
\hline \multirow[t]{4}{*}{2} & ATP Binding & & & \\
\hline & PA1393_cysC_at & Adenylyl-sulfate kinase & \multicolumn{2}{|c|}{ Enrichment Score: 0.03} \\
\hline & PA1386_at & Serine O-acetyltransferase & $8.60 \mathrm{E}-01$ & $1.00 \mathrm{E}+00$ \\
\hline & PA4526_pilB_at & Type 4 fimbrial assembly protein PilB & & \\
\hline
\end{tabular}


In general, according to the functional clusters, internalised $P$. aeruginosa looked to have down-regulated systems required for cell detoxification, DNA repair and motility (potential virulence factor) and up-regulated systems that repress the SOS DNA damage response (LexA repressor), protect from protein aggregates, and signal alginate production. Unlike previously conducted in vitro microarray studies with $P$. aeruginosa and $\mathrm{H}_{2} \mathrm{O}_{2}$ (Table 4.1), the catalase genes, katA, katB and katE, did not make the 3 fold up-regulation cut off for any of the samples. Indeed, all three catalase genes were down-regulated $(-1.25,-1.99$, and -1.53 respectively) which is in direct contrast to published microarray data sets that attempted to model the infectious state by in vitro challenge with $\mathrm{H}_{2} \mathrm{O}_{2}(78,197-199)$. This result was particularly surprising given that KatA has on several accounts been characterised as essential for resistance, osmoprotection and virulence $(111,112)$.

\subsubsection{Expression data cross referenced with annotated operons in DOOR}

Overall the functional clusters above did not indicate many clear themes in up- or down-regulation despite key genes like the lexA SOS repressor and algU alginate sigma factor being represented in functionally-related gene groups. This may be a consequence of overly stringent parameters for recognition of altered expression; however, relaxing these parameters substantially rapidly leads to overwhelming levels of confounding "noise" in the data sets.

As an alternative approach to infer biological relevance from the microarray data, a cross referencing of the expression data with predicted operons in the DOOR database was performed. Using this approach, inclusive of all expression values regardless of fold change, it was revealed that operons or predicted operons were upregulated intermittently throughout the genome (some regions with as many as 110 contiguous genes). Additionally, many of the transcription regulators of the identified operons (as defined by Galán-Vásquez et al (2011; (240)) were also found to be upregulated. In contrast to the functional clustering by DAVID, identification of transcription regulators and their associated operons using the DOOR database seemed to paint a clearer picture of the overall response by the bacterium. Table 4.6 lists the transcriptional regulators and their target(s) as found in the Internal PAO1 upregulated data set. 
Table 4.6. Transcriptional regulators (Internal PAO1 up-regulated data set)

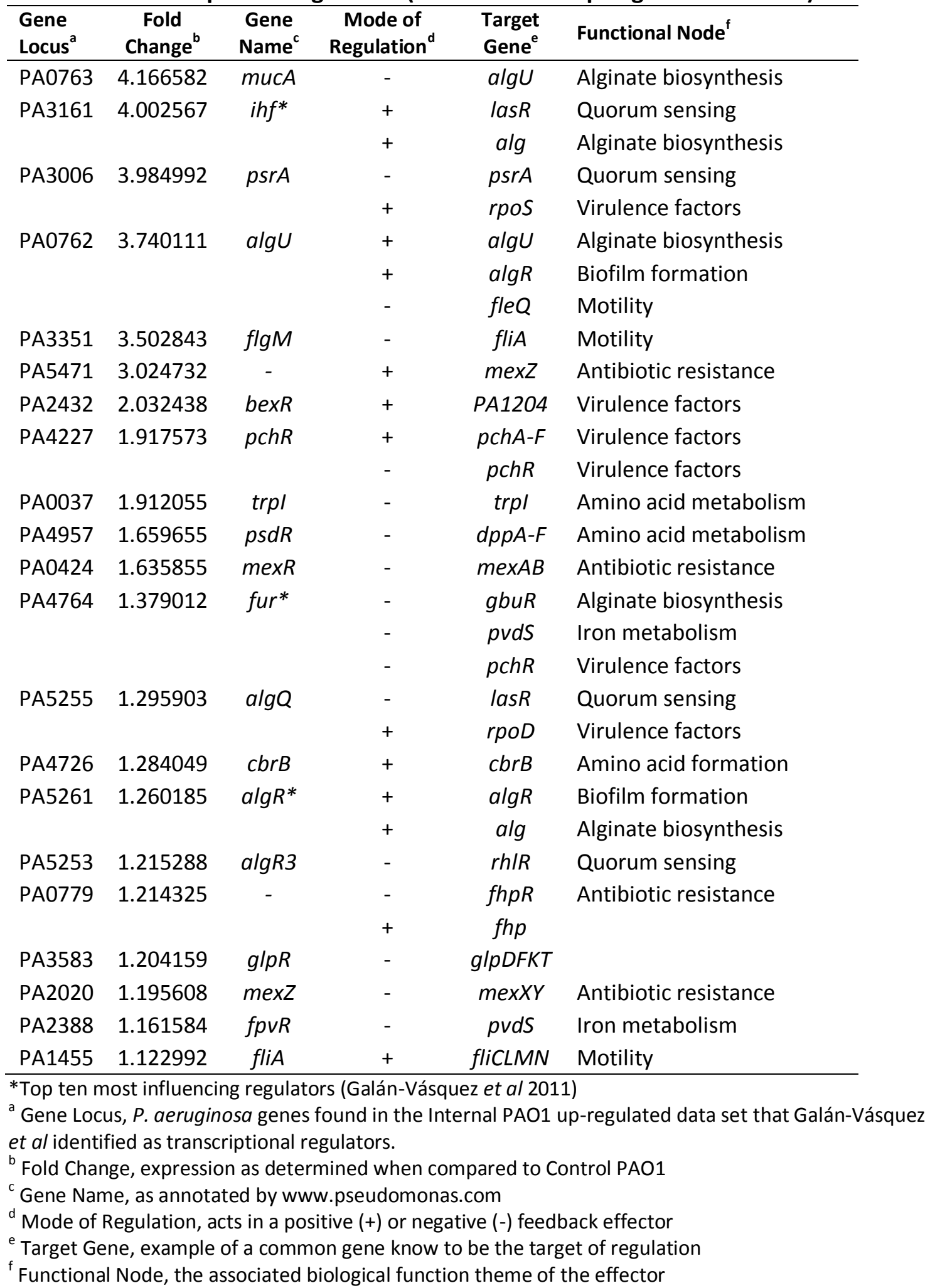




\subsubsection{The three P's: Pyocin, Pyoverdine, and Pyochelin}

Pyocin, a secreted bacteriocin of $P$. aeruginosa, is synthesised when the bacterium encounters the presence of a mutagen such as ROS produced by macrophages (241). Pyocin activation is also known to be a result of increased recA expression or the pyocin activator prtN $(242,243)$. In the event of pyocin production, the bacterium would also be expected to up-regulate the imm 2 immunity protein rendering $P$. aeruginosa protected from its own bacteriocin production $(243,244)$. However, despite both recA and prtN being up-regulated greater than two-fold in both Internal PAO1 and External PAO1 data sets, the entire R-and F-type pyocin ORFs, including the imm2 and S-type pyocin-containing operons, were either down-regulated or not present in the expression data.

Also known to be involved in pathogenesis are the siderophores pyoverdine (Pvd) and pyochelin (Pch) which aid in sequestering iron from the intercellular iron stores in eukaryotic hosts for the purpose of supporting bacterial growth $(245,246)$. Although Pvd has been characterised as critical for $P$. aeruginosa virulence, literature suggests that the role of the Pch siderophore in pathogenesis is minor due to the observations that Pvd-deficient Pch-rich strains exhibit significantly slowed growth under iron limitation, but not vice versa (247-249). Relative to the Control PAO1, the Pvd synthesis genes (PA0610 - PA0650) were repressed in the Internal PAO1, and conversely activated in the External PAO1. In contrast, the $p c h A-F$, flanking operons, including the $p c h$ receptor $f p t A$ and regulator $p c h R$, were activated in the Internal PAO1.

Both the macrophage and bacteria would be expected to produce siderophores and compete with one another for limited iron to support growth $(250,251)$. The host protects its iron by a) storing it intracellularly, and b) binding it to transferrin which can also be used to transport the iron (i) to other host cells, or (ii) into the phagosome where excess amounts of iron contribute to oxidative stress and antimicrobial activity via Fenton chemistry (252-256).

The observation that Pvd was up-regulated in the External PAO1 sample is consistent with previous literature, as Pvd has been shown to acquire iron bound to transferrin during extracellular transport between macrophages $(247,257-260)$. It also makes sense that $P$. aeruginosa might prefer to not activate Pvd within the phagosome 
due to Fenton chemistry (rapid iron-catalysed $\mathrm{H}_{2} \mathrm{O}_{2}$ conversion to the hydroxyl radical). However, there is little in the literature that would indicate an intracellular role for Pch, although extracellularly produced Pch has been shown to be able to enter the cytoplasm (261). In in vitro pulmonary infection studies, Pch has also been implicated in intensifying oxidative damage leading to endothelial cell damage (262-264). Secondary siderophores of Pseudomonas species have previously been implicated in both defensive and virulence roles unrelated to their iron-transporting capabilities (265-267), and it may be that the apparent up-regulation of Pch production in the Internal PAO1 cells reflects a similar role for Pch in this infection model. 


\subsubsection{Oxidoreductases activated by phagocytosis}

One of the original aims of this project was to identify NQORs that may play a role in protecting $P$. aeruginosa from oxidative stress, in particular ROS generated during phagocytosis by immunocompetent cells such as macrophages. Firstly, and irrespective of operons, two hypothetical NQORs were significantly down-regulated in Internal PAO1: PA0804 (-1. 79 FC) and PA2580 (-3.02 FC) with B-scores of 5.8 and 5.6 respectively. PA2580 shares $56 \%$ protein sequence identity with the MdaB homologue in $\mathrm{H}$. pylori which has been shown to confer oxidative stress resistance in the animal model (128). In our over-expression studies (Figure 4.14B), we also demonstrated that PA2580 had potential to defend against $\mathrm{H}_{2} \mathrm{O}_{2}$, however, the native enzyme does not appear to be playing such a role in the in vivo cell culture infection model.

In contrast, twelve hypothetical NQORs (characterised and putative) were activated in the Internal PAO1 when compared to Control PAO1. Genes were determined to be a putative NQOR by shared protein identity (as defined in Chapter 3) and by annotation at www.pseudomonas.com which indicated a cytoplasmic location for the gene product and an $\mathrm{NAD}(\mathrm{P}) \mathrm{H}$ dependency. Additionally, 5 of these redox genes (PA2906, PA3208, PA3567, PA4975, and PA4986) were found to be within predicted operon regions and to possess B-scores greater than 2 (log odds that the gene is differentially expressed where a score of $>0=50 \%$ probability and $>3=95 \%$, Table 4.7). Furthermore, several of the operons were surrounded by contiguous sequences of up-regulated genes. Although many of these adjacent genes are hypothetical, it is possible that these operon-adjacent sequences may be biologically related as bacterial genomes often have functionally related genes located near each other. 
Table 4.7. Putative NQORs identified in Internal PAO1 up-regulated operons

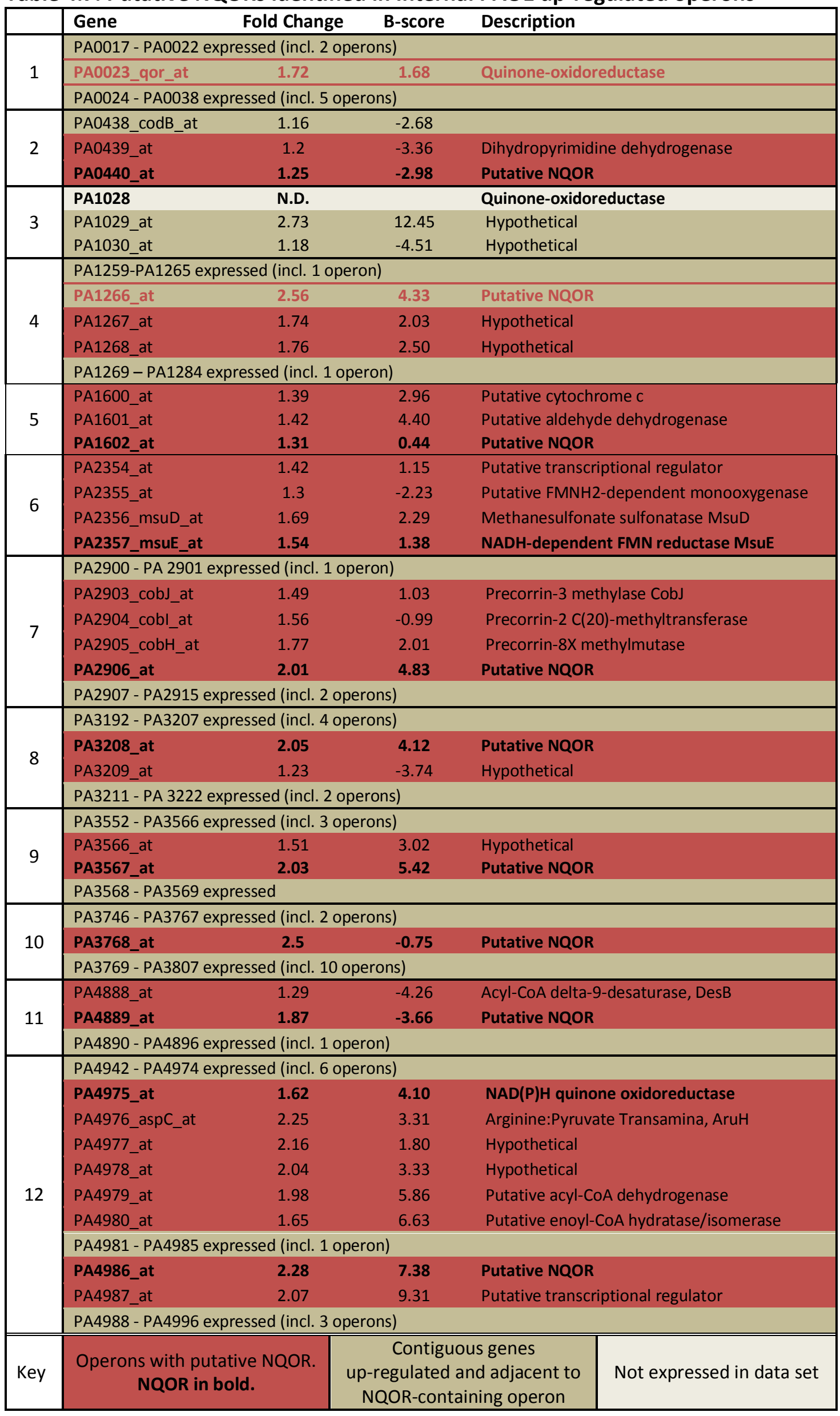




\subsubsection{Miscellaneous observations}

$P$. aeruginosa is capable of hydrogen cyanide biosynthesis during infection, and cyanide, a potent inhibitor of mitochondrial respiration, is known to promote or even be a dominant factor in host cell killing $(58,268)$. During human infection it may be that cyanide production impacts macrophages more severely than neutrophils because macrophages have more mitochondria $(62,269,270)$. Although Internal PAO1 did not appear to activate the cyanide biosynthesis operon (hcnABC), the rhdA-psd (PA4954$4957)$ operon, which catalyses detoxification of cyanide $(271,272)$, was strongly upregulated $(\mathrm{PA} 4954=+2.16 \mathrm{FC}$, and PA4957 $=+1.66 \mathrm{FC}$ with B-scores of 8 and 4 respectively).

$P$. aeruginosa cyanide production has been shown to be controlled by several regulators such as the master regulator $\operatorname{AlgR}(240,273)$ and the anaerobic regulator ANR (274), but most importantly by the quorum-sensing regulators LasR and RhIR $(274,275)$. As with cyanide biosynthesis, quorum sensing can also play an important role in virulence. Two operons known to be activated by quorum-sensing stood out in the Internal PAO1 data set. These two operons (PA2301-PA2305 and PA3327-PA3333) have both been predicted to be activated during quorum sensing (276), and both contain non-ribosomal peptide synthetase (NRPS) genes. NPRSs are large enzymes that condense amino acids in an assembly-line fashion and are of great interest due their role in generating a wide variety of biologically active metabolites (277). Though no literature could be found identifying the end-product of the PA3327 NRPS, one reference was found that indicated preliminary characterisation of the PA2302 NRPS. A doctoral thesis by Yang Fan (2008, National University of Singapore) proposed that the PA2302 NRPS is required to make an endogenous quorum sensing inhibitor "tentatively named PAi", which provides feedback regulation of quorum sensing (278). In support of the "PAi" inhibitor and the up-regulation of the PA2302 NRPS, the quorum sensing regulators LasR and RhIR were both down-regulated in the Internal PAO1 data set (-1.82 FC and -1.20 respectively and both with B-scores of 2 ). 


\subsection{Discussion}

In the 1995 edition of Free Radicals in Biology and Medicine, there was a call to move away from studies of isolated cell systems under non-physiological conditions and from reactions involving constituent $\mathrm{H}_{2} \mathrm{O}_{2}$ in media (70). Although our study is in some respects still an "isolated cell system", we believe it was a positive step in the direction of studying complex $P$. aeruginosa interactions by simulating an in vivo environment for the bacteria, as opposed to in vitro $\mathrm{H}_{2} \mathrm{O}_{2}$ stimulation in nutrient rich culture media as per the studies noted in Table 4.1. We were interested primarily in how $P$. aeruginosa tolerates the oxidative stress encountered during initial contact with the innate immune system; therefore we used established macrophage-like cell lines to optimise a flow cytometry-based method for infecting and recovering viable $P$. aeruginosa that had been engulfed by macrophages. We used this method to recover P. aeruginosa RNA from viable bacteria located within the macrophage, a technical accomplishment that has not previously been reported.

A robust and reliable infection model was developed by successive evaluation of different parameters of infection, selecting the optimal cell line to be infected, a suitable bacterial $\mathrm{MOI}$, whether the bacteria should be opsonised, and the influence that different macrophage stimuli (IFN- $\gamma$ ) have on uptake of $P$. aeruginosa. This advancement in $P$. aeruginosa specific techniques also allowed for the ability to assess multiple gene deletion and over-expression strain variants in parallel, by employing flow cytometry-base enumeration in a high-throughput style assay.

Using the in vivo cell culture infection model, the NQOR over-expression strains, $\triangle k a t A+p S X:: P A 2580$ and $\triangle k a t A+p S X:: P A 0949$, were found to be less sensitive to challenge by activated macrophages than controls. This observation was similar to results obtained by $\mathrm{H}_{2} \mathrm{O}_{2}$ challenge in vitro (Figure 4.15 and section 3.3.2.2). Similarly the NZ clinical isolates ARL1079, ARL1205, and ARL1236 demonstrated no substantial difference in survival when compared to wild type PAO1 in the in vivo cell culture model. This too reflected observations made with in vitro $\mathrm{H}_{2} \mathrm{O}_{2}$ challenge. Moreover, while some of the NQOR over-expression strains or clinical isolates behaved in a consistent fashion in each of the two stress models, some additional NQOR overexpressing or gene-deleted strains that did not appear to differ from the wild type control in in vitro $\mathrm{H}_{2} \mathrm{O}_{2}$ assays did now exhibit a phenotypic difference from wild type 
in response to macrophage challenge. For example, in Chapter 3, the $P$. aeruginosa mutant $\Delta 1204$ did not exhibit heightened sensitivity to $\mathrm{H}_{2} \mathrm{O}_{2}$ even in the absence of the katA gene. Conversely, in the in vivo cell culture model, the $\Delta 1204$ mutant exhibited sensitivity to macrophage infection with katA present. Although any conclusions to be drawn at this stage remain highly speculative, these minor phenotypic differences may suggest that PA1204 does play some role in virulence. Additional support for this hypothesis comes from the observation that the bex $R$ virulence regulator was also upregulated in the Internal PAO1 cells, as BexR is known to positively regulate the 1204 operon $(240,279)$.

In similar fashion, the $\triangle k a t A$ gene deletion both conferred sensitivity to in vitro $\mathrm{H}_{2} \mathrm{O}_{2}$ challenge (section 3.3.2.1) as well as reducing survival to macrophage infection (section 4.5.2). Surprisingly however, during the transcriptional response of wild type PAO1 to macrophage infection (section 4.7.2) levels of katA gene expression as well as katB and katE were down-regulated. One possible explanation for the down-regulation these catalases, as well as other known virulence factors such as pyoverdine and the $m d a B$ orthologue (PA2580), is that $P$. aeruginosa has previously been found to turn off essential virulence determinants in the presence of various stressors thus accelerating its rate of hypermutability (280-282). It has also been reported that mutants lacking DNA mismatch repair systems, such as mutS, mutL, or uvrD, significantly contribute to hypermutation rates found at high frequencies in chronic lung infections $(280,283,284)$. The down-regulation of virulence determinants such as katABE, PA2580, and pyoverdine related genes supports the idea that $P$. aeruginosa is reducing its defence mechanisms in the presence of macrophages to (possibly) enhance the rate of mutation accumulation.

An alternative argument is that the down-regulation of katABE and pyoverdine may be indicative of a need by $P$. aeruginosa to restrict intracellular iron which, as a result, limits the ability of the bacteria to produce these catalases. The reason for this may be that the bacterial cell can "cope" with superoxide and $\mathrm{H}_{2} \mathrm{O}_{2}$ challenge, but generation of substantial levels of the particularly damaging hydroxyl radical species from $\mathrm{H}_{2} \mathrm{O}_{2}$ via iron-catalysed Fenton chemistry might prove too much of a challenge for bacterial survival. This argument is not entirely inconsistent with the "hypermutation" hypothesis presented in the previous paragraph; rather it is just suggesting that $P$. 
aeruginosa may need to carefully control the balance between too little and too much stress-induced damage.

Furthermore, several studies have indicated that $P$. aeruginosa may antagonise macrophages into producing ROS for extended periods thus manipulating the inflammatory response and promoting programed cell death of the macrophage and other phagocytic cells (285-288). It has been well documented that $P$. aeruginosa stimulates the production of ROS by activating the host cell's NADH oxidase $(285,286)$. In early stages of infection, oxidant/antioxidant balance is maintained by the host, however, under prolonged exposure to ROS and the resulting ROS accumulation, the host cell undergoes bacteria-induced apoptosis (287-290). It may therefore be that $P$. aeruginosa-generated ROS in tandem with the down-regulation of DNA repair systems has a two-fold value in that (i) it allows for accumulation of mutations that may bring about beneficial evolutionary changes, and (ii) it provides a means to counter the macrophage defence.

Based on the literature, it also seems possible that $P$. aeruginosa may be preferentially driving uptake of itself by macrophages over neutrophils. Neutrophils, recruited by the presence of $P$. aeruginosa (291), have been shown to be essential for $P$. aeruginosa clearance during infection (292). However, $P$. aeruginosa can interfere with neutrophil function by (i) masking complement deposition through the production of the Psl protein during initial attachment (293-295), (ii) reducing $P$. aeruginosa uptake and ROS production by neutrophils (17), and (iii) stimulating neutrophil apoptosis $(92,211,296-298)$. Additionally, mannose receptors of macrophages facilitate phagocytosis of non-opsonized $P$. aeruginosa (299) and Psl is mannose-rich (300) which brings the story full circle to $P$. aeruginosa preferentially driving uptake of itself by macrophages.

To further elucidate the biological significant of the NQOR PA1204, and other NQORs, or the down-regulation of katABE in Internal PAO1 would require not only quantitative RT-PCR validation of expression levels, but also an extensive investigation into the influence of $P$. aeruginosa population density and macrophage exposure time. As demonstrated in section 4.4.4, P. aeruginosa in the presence of murine macrophages behaved differently at MOIs of 50 and 100 indicating that population density influences the bacterial response. Levels of survival of PAO1 also changed with time. These are important points to consider given that all of the studies in Table 4.1 
used different $P$. aeruginosa densities and $\mathrm{H}_{2} \mathrm{O}_{2}$ challenge times for their investigations, and consequently saw quite different transcriptional profiles. It is well established that $P$. aeruginosa intercellular communication is critical to virulence and that quorum sensing not only senses, but also induces major cellular responses to variations in population density $(2,301)$. A model to elucidate virulence factors contributing to pathogenesis should be able to take this into consideration and with the in vivo cell culture model described above, it is now possible to investigate individual parameters side-by-side in a high-throughput fashion.

While these experiments demonstrated the potential for screening $P$. aeruginosa strains en masse, robust validation through further in vitro and in vivo study should be undertaken before concluding any biological roles for specific NQORs as unexpected results emerged intermittently throughout optimisation. Fluctuations in the bacterial incubation times could easily distort the number of viable bacteria recovered, as could undesirable temperature changes during the extensive wash steps for the phagosomal maturation studies. Additionally the changing "age" of the immortal RAW-264.7 macrophages made it difficult to directly compare bacteria cell numbers between experiments conducted at distant time periods. Variations of cell density and the culture surface of the immortal RAW-264.7 cells also influenced the survival of PAO1. Although we aimed to accommodate many of these factors by instigating rigorously timed protocols, we cannot rule out that unaccounted fluctuations in some or all of these parameters may have influenced some results.

We also hypothesise that this in vivo cell culture method will likely have sufficient flexibility to be adapted for evaluation of the $P$. aeruginosa response to challenge by other phagocytic cell types (e.g. neutrophils) as well as to gauge, in tandem, how the eukaryotic host acts in response to the bacteria by employing established immunological assays. For example, this system might be used to address how common chemical signals in the innate immunological response, like IFN- $\gamma$, can affect the $P$. aeruginosa regulation of virulence factors via the transcriptional response. This may enable further elucidation of how pathogens like $P$. aeruginosa control production of specific virulence factors and identification of key genes involved in pathogenesis. Thus, this infection model has many more potential applications, ranging from pure microbiological studies to investigations into immunological and host-pathogen interactions. 
One of the major caveats of studying bacterial transcription, however, is the instability and rapid half-life of microbial mRNAs (188-192). With bacteria able degrade expressed RNA in less than 2 minutes, and sometimes even mid translation, methods of prokaryotic RNA isolation need to be highly efficient to prevent artefactual results (191,193-195). Thus, any global microbial expression profiles should be carefully considered in the context of their specific experimental design.

In future applications it could be advantageous to incorporate the use of primary cell lines into this infection model. Although we chose to use the established RAW-264.7 cell line for the optimisation of this infection model, the heterogeneous nature of primary cells would most likely have yielded a different transcriptional response by $P$. aeruginosa. Additionally, had sufficient funds been available, it would have been interesting to compare the transcriptional response to primary cell lines by PAO1 over time, to address questions raised in section 4.4 .3 where internalised PAO1 viability changed with time (Figure 4.7 and Figure 4.8). It would also be interesting to compare the laboratory strain PAO1 with the response of NZ clinical isolates to gauge how appropriate the universal PAO1 type strain is for modelling infection. However, microarray analysis is expensive and financial constraints precluded us from considering more than three samples (Internal PAO1, External PAO1, and Control PAO1) to address the basic question of "what genes do $P$. aeruginosa activate when engulfed by macrophages?"

Whilst our study perhaps raises more questions than answers in regard to the $P$. aeruginosa response to challenge by activated macrophages, it nonetheless provides a clear benchmark for future studies to be compared against, as well as contributing a valuable tool with many potential applications for understanding of the greater biological process encompassing the establishment of disease. Current trends in immunology appear to be leaning towards interdisciplinary approaches to investigating host-pathogen interactions; and although macrophage responses to bacterial pathogens have been well characterised, the advancement of microarray technology and the addition of in vivo cell culture models now lend valuable insights as to how the bacteria are in turn responding to macrophages. This is a different approach to the same question and perhaps reflects a changing approach to identification of therapeutic targets for bacterial infections like those caused by $P$. aeruginosa; instead of exploiting immune cell strategies to prevent disease, we are 
now investigating what the bacteria do to cause disease. Now that more experimentation is being conducted on whole systems (addressing the interaction of overlapping virulence systems), therapeutic target identification is moving beyond isolated constituent stressor studies to a more complex map of crosstalk between host and pathogen in the presence of naturally produced immune compounds in physiologically correct conditions. 


\section{Chapter 5: Activation of the nitro-chloromethyl benzindoline prodrug SN 26438 by P. aeruginosa NfsB-like NQOR: A novel enzyme-prodrug pairing for GDEPT}

\subsection{Introduction}

During the course of this research we had the opportunity to test the reductive capacity of $P$. aeruginosa NQORs with a promising next-generation prodrug for GDEPT, and saw some very promising preliminary results. GDEPT is a targeted approach to cancer management wherein tumour cells are sensitised, via selective transgene delivery, to a systemically administered prodrug. An important characteristic of GDEPT is the bystander effect - the ability of activated prodrugs to transport either passively or actively out of the cell of origin, and into neighbouring, non-transfected cells - as this provides an elegant solution to the unavoidable issue of low cell transfection rates, historically viewed as a key limitation of GDEPT $(302,303)$. Studies employing prodrugs with high bystander effects have demonstrated that significant tumour reduction can occur when less than $0.1 \%$ of the tumour population expresses the activating enzyme (304).

Therapeutic genes employed in GDEPT are typically non-human in origin, to minimise the potential for prodrug activation by endogenous enzymes in healthy tissue. Two of the most widely studied genes for GDEPT are herpes simplex virus thymidine kinase, which was evaluated in phase III clinical trials in combination with the nucleotide analogue prodrug ganciclovir (305); and cytosine deaminase from Escherichia coli, which has undergone phase I trial in combination with another nucleoside analogue prodrug, 5-fluorocytosine (306). These systems enjoy the substantial benefit that their prodrugs have each achieved independent clinical utility; but they also suffer from the limitations that only actively dividing cells are targeted, that their activated metabolites inhibit replication of viral vectors, and that their cellto-cell bystander effects are largely dependent on functional gap junctions, which many tumour cells lack $(302,303,307,308)$.

A third enzyme-prodrug combination that has undergone clinical evaluation for GDEPT is E. coli NfsB in combination with CB1954 [5-(aziridin-1-yl)-2,4- 
dinitrobenzamide] $(309,310)$. Successive 2-electron reductions of CB1954 by NfsB results in activated metabolites that can freely diffuse across cell membranes, forming adducts and DNA crosslinks and inducing apoptosis in both replicating and quiescent tumour cells $(136,141,142,311)$. Nonetheless, results of the clinical trials were somewhat equivocal - while there was evidence for mild anti-tumour effects, CB1954 was also discovered to exhibit dose-limiting hepatotoxicity $(309,310)$. In our on-going research we have sought to improve the efficacy of CB1954 GDEPT through identification and engineering of nitroreductases that exhibit superior activity to $\mathrm{NfsB}$ at concentrations of prodrug that are attainable in vivo $(139,312,313)$. However, we have also found that CB1954 exhibits a relatively modest bystander effect relative to other nitro-aromatic prodrugs with potential utility in GDEPT (this work; (314)). Thus, we have a strong interest in identifying superior prodrug substrates for nitroreductase GDEPT.

One class of bioreductive prodrugs that is of particular interest is the nitrochloromethylbenzindolines (nitro-CBIs). These compounds were designed as hypoxiaactivated prodrugs of the cyclopropylindoline anti-tumour antibiotics, exemplified by CC-1065 and duocarmycin SA (315). The potent cytotoxicity of the cyclopropylindoline antibiotics is due to sequence selective alkylation of the N3 of adenine in the minor groove of DNA, generating 'stealth lesions' that are not easily repaired (Figure 5.1; $(315,316))$. The nitro-CBI prodrug analogues of these molecules were generated by replacing the key phenol of the alkylating subunit with nitro functional groups, which can be enzymatically reduced to the DNA alkylating form (317). As the primary human reductases for these molecules act via successive one electron steps, the presence of molecular oxygen establishes a futile reduction cycle, conferring hypoxia-selectivity to these prodrugs. In contrast, $\mathrm{NfsB}$ reduces these molecules via oxygen-independent two electron steps, yielding end metabolites that exhibit substantial bystander effects (317). This finding suggests that the nitro-CBIs are likely to prove effective prodrugs for nitroreductase GDEPT. 
A

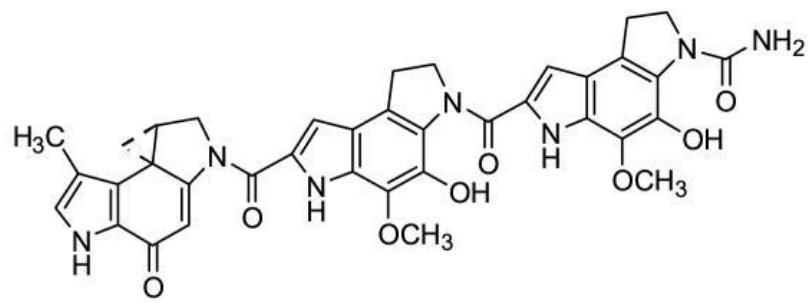<smiles>COc1cc2cc(C(=O)N3CC[C@]4([Al])C3=CC(=O)c3[nH]c(C(C)=O)cc34)[nH]c2c(OC)c1OC</smiles><smiles>CCCCOc1ccc2[nH]c(C(=O)N3CC(CCl)c4c3cc([N+](=O)[O-])c3ccccc43)cc2c1</smiles>

Duocarmycin SA

SN 26438

B

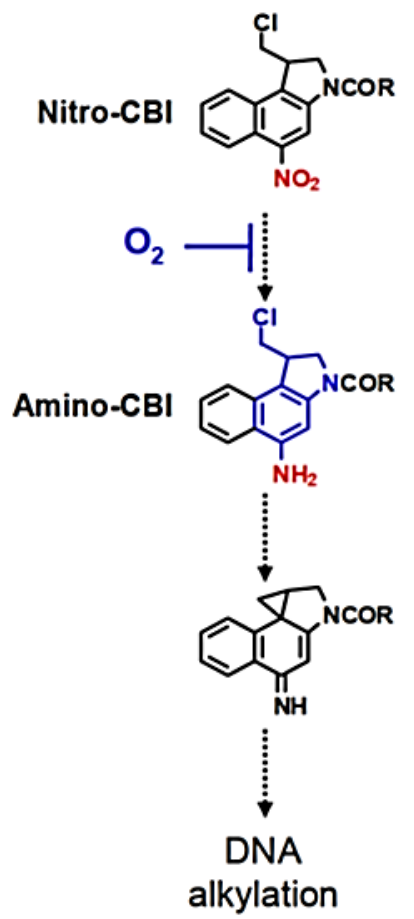

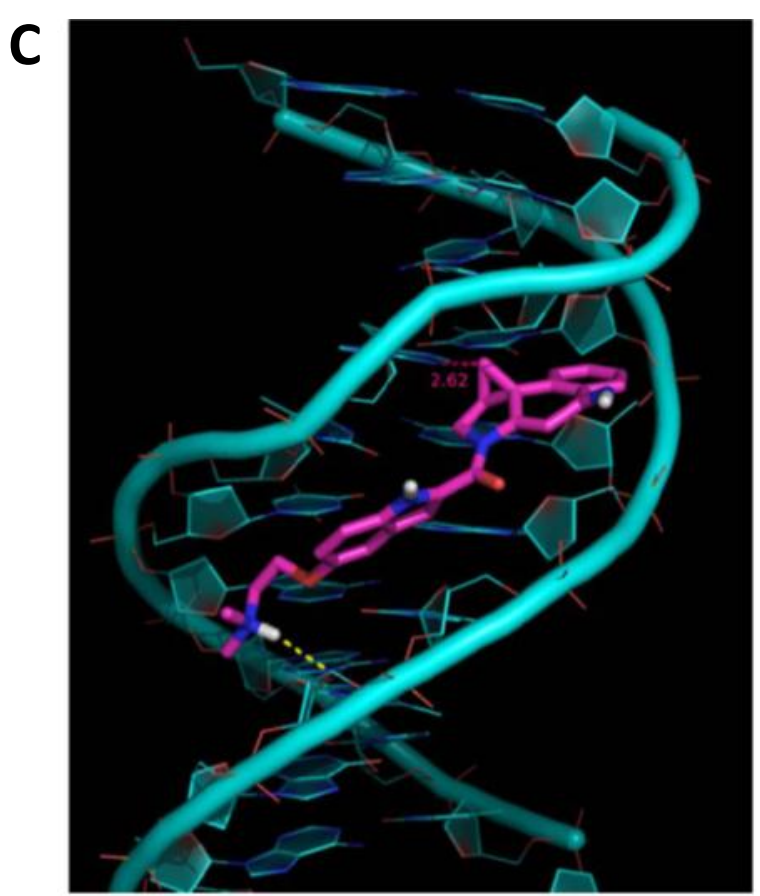

\section{Figure 5.1. Nitro-CBIs}

(A) The structure of two anti-tumour antibiotics and their nitro-CBI analogue, SN 26438. (B) Metabolic activation of nitro- $\mathrm{CBI}$ and $(\mathrm{C})$ computer modelling example of the interaction between the activated nitro-CBI metabolite with adenine. Panels (B) and (C) were reproduced with permission from Dr Sophie Syddall's University of Auckland PhD thesis (318). 
This chapter describes our efforts to identify a suitable nitroreductase to partner with the lead nitro-CBI prodrug, SN 26438 (also known as nitro-CBI-DEI (317)). Preliminary tests indicated that some of the $P$. aeruginosa NQORs were substantially more active with SN 26438 than the previous "gold standard" GDEPT nitroreductase, $E$. coli NfsB, and thus the $P$. aeruginosa NQORs became the focus of a detailed characterisation of their activities with SN 26438 as well as CB1954.

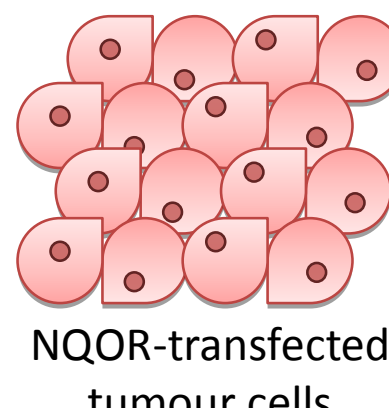

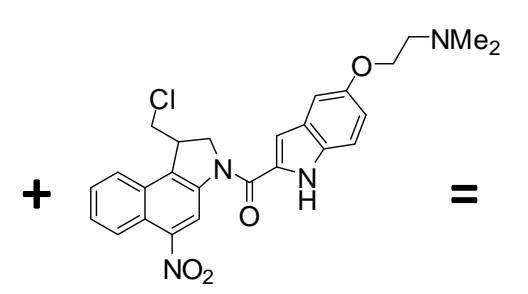

Prodrug

SN 26438

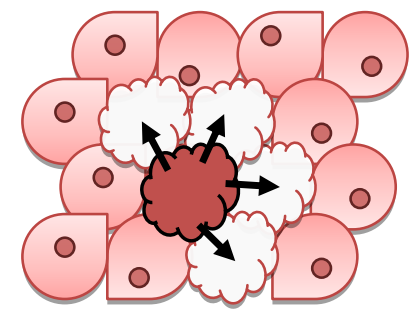

Bystander

Killing

\section{Figure 5.2. Schematic representation of the bystander effect}

Prodrug activated by a single NQOR-expressing tumour cell can be transported either passively or actively out of the cell of origin, and into neighbouring, non-transfected cells, killing them also.

\subsection{Objectives}

- Screen the $P$. aeruginosa NQORs for activity with CB1954 and SN 26438 relative to E. coli NfsA and NfsB.

- Develop a novel model system for measuring the ability of preferred NQOR candidates to sensitise human tumour cells to prodrugs without a requirement for generating stably transfected cell lines.

- Determine the bystander effect of SN 26438 in combination with a preferred NQOR activating enzyme. 


\subsection{Results}

\subsubsection{Evaluation of CB1954 and SN 26438-reducing activity of candidate $P$.} aeruginosa NQORs through their ability to induce the $E$. coli SOS response

Candidate $P$. aeruginosa NQORs were selected on the basis of shared amino acid sequence identity (>25\%) to previously identified $E$. coli nitroreductase candidates (139) and evaluated for CB1954 and SN 26434-reducing activity (Table 5.1). A total of $12 P$. aeruginosa NQOR candidates were selected whose $E$. coli orthologues had previously demonstrated various degrees of reductive capacity when partnered with the prodrug CB1954 (13).

Table 5.1. Candidate $P$. aeruginosa NQOR Selection

\begin{tabular}{llll}
\hline Gene Locus & \% Identity $^{a}$ & $\begin{array}{l}\text { Closest E. coli } \\
\text { Orthologue }\end{array}$ & Accession Number \\
\hline PA5190 & 25 & NfsB & AP_001223 \\
PA2357 & 26 & SsuE & AAC_74023 \\
PA0949 & 39 & WrbA & AP_001635 \\
PA2580 & 64 & MdaB & AP_003578 \\
PA3223 & 45 & AzoR & AP_002037 \\
PA1962 & 41 & AzoR & AP_002037 \\
PA3208 & 42 & Ydja & AP_002384 \\
PA1204 & 45 & YieF & AP_004074 \\
PA4975 & 37 & KefF & AP_000710 \\
PA0853 & 27 & YcaK & AP_001531 \\
PA1225 & 33 & YcaK & AP_001531 \\
PA2932 & 52 & NemA & AP_002272 \\
\hline
\end{tabular}

${ }^{A}$ Shared amino acid identity with the closest $E$. coli ortholouge.

${ }^{\mathrm{B}} E$. coli gene orthologue accession number in the NCBI protein databases.

The selected $P$. aeruginosa NQOR candidates were first tested for the ability to activate CB1954 or SN 26438 in E. coli SOS assays, a quantitative system for measuring the level of activation of genotoxic prodrugs that was previously developed in the Ackerley laboratory. The basis of the SOS assay for prodrug activation is that candidate nitroreductases are cloned into a custom-made expression plasmid ( $\mathrm{PUCX}$ ) and overexpressed in a strain of $E$. coli that has a lacZ reporter gene under control of an SOS responsive promoter (139). This strain is then challenged with sub-lethal levels of a nitro-aromatic prodrug, whereupon nitroreductase activity causes DNA damage, 
inducing the SOS (DNA damage repair) response and production of quantifiable levels of $\beta$-galactosidase. For this study the $E$. coli reporter strain SOS-R2 was used. Whereas the first generation reporter strain SOS-R1 had the native $n f s A$ and $n f s B$ genes deleted from its genome (139), SOS-R2 contains additional nemA and $a z o R$ gene deletions also, as these have been shown to lower background levels of prodrug activation in empty plasmid control cells (Dr David Ackerley, personal communication). Moreover, SOS-R2 also contains a tolC gene deletion. TolC is an outer membrane pore protein that plays a key role in expulsion of some toxins and xenobiotics from the cell (319), and tolC gene deletion has previously been found to improve SOS assay sensitivity for some genotoxic compounds (320,321). Additionally, work by former lab member, Dr Gareth Prosser, exetensively demonstrated that NQORs from a wide range of bacterial hosts are expressed at similar levels in the E. coli SOS-R2 strain $(139,322)$.

The selected $P$. aeruginosa NQOR genes were individually cloned into pUCX and over-expressed in SOS-R2 alongside an empty plasmid negative control and $E$. coli nfs $A$ and $n f s B$ positive controls (Figure 5.3). When these strains were challenged with 20 $\mu \mathrm{M}$ CB1954, both the NfsA and NfsB positive control strains exhibited a strong (ca. 5fold) increase in SOS response relative to the empty plasmid control strain (as measured in Miller Units of LacZ activity resulting from induction of the lacZ reporter gene; Figure 5.4A). However, of the P. aeruginosa NQOR candidates, only PA5190 exhibited a small (2.3-fold) increase in SOS activity with CB1954. In contrast, challenge with $5 \mu \mathrm{M}$ SN 26438 generated a strong (> 11-fold) induction of SOS response by two P. aeruginosa NQORs as well as E. coli NfsB and NfsA (Figure 5.4B).

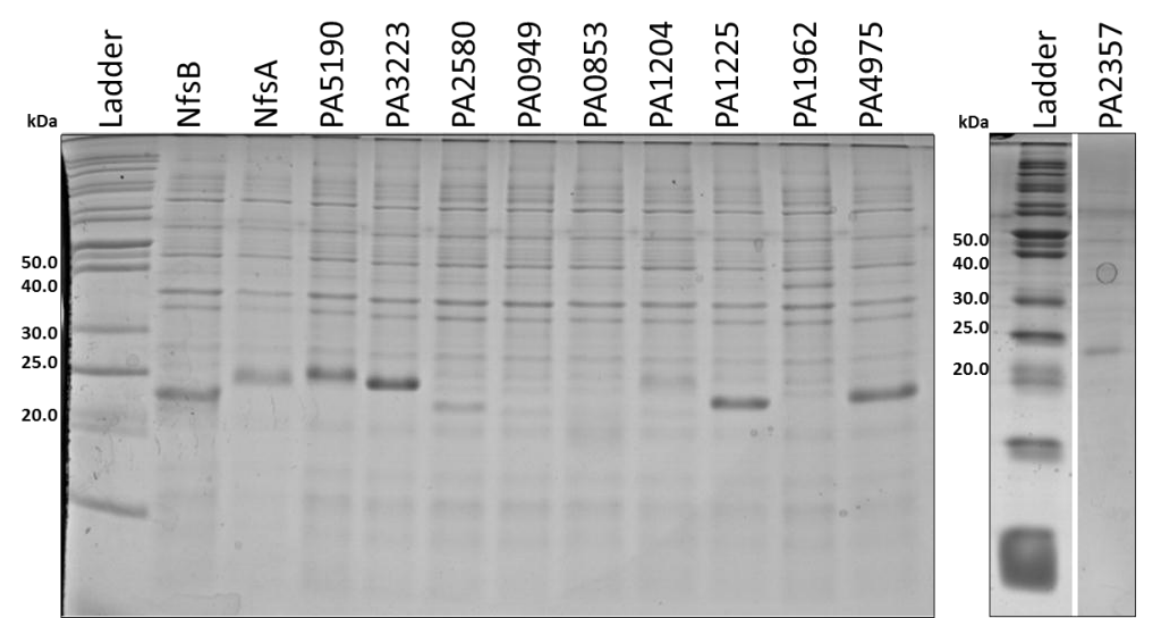

Figure 5.3. Expression of $P$. aeruginosa NQORs in SOS-R2

SDS-PAGE analysis of expression levels of $P$. aerufinosa NQORs from pUCX in SOS-R2. Samples for electrophoresis were taken from a single replicate at 3 hours of IPTG induced expression. Samples were normalised for cell density $\left(O D_{600}\right)$ prior to gel loading. 
A

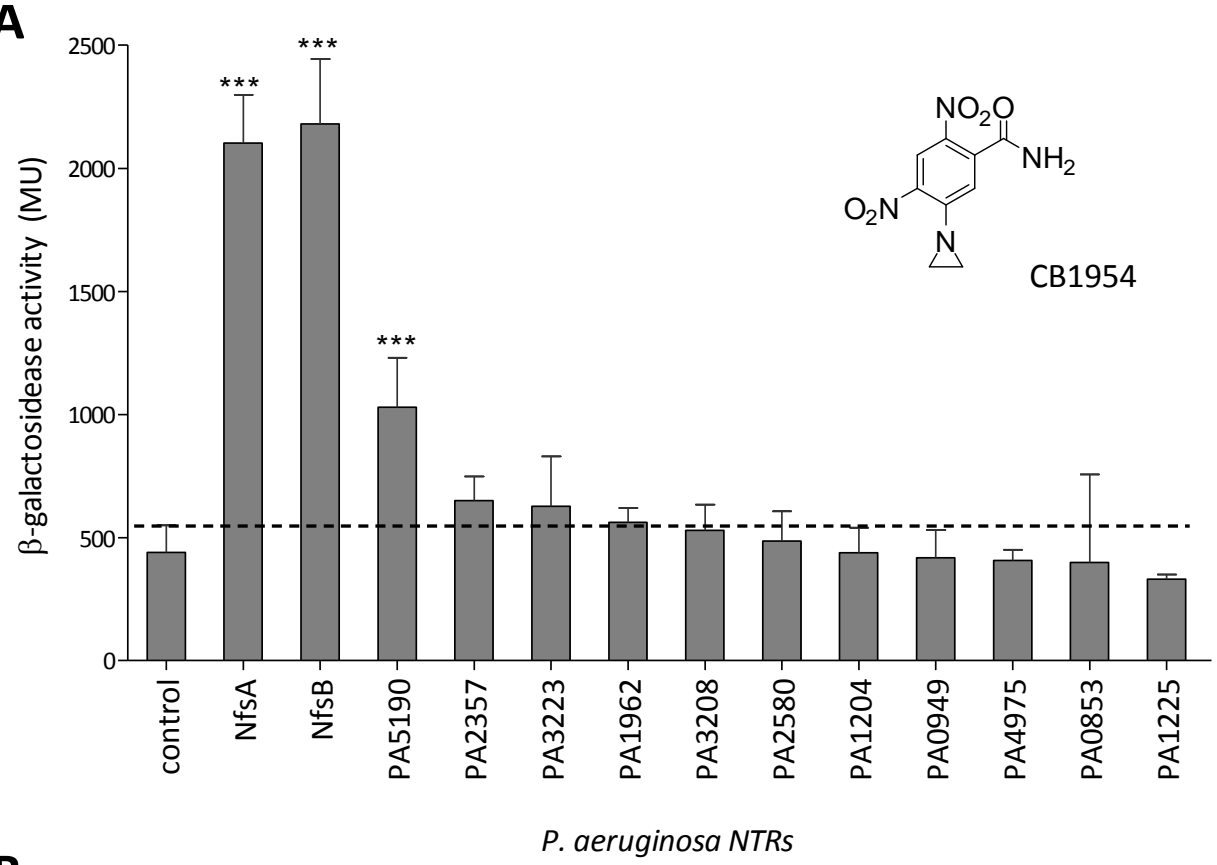

B

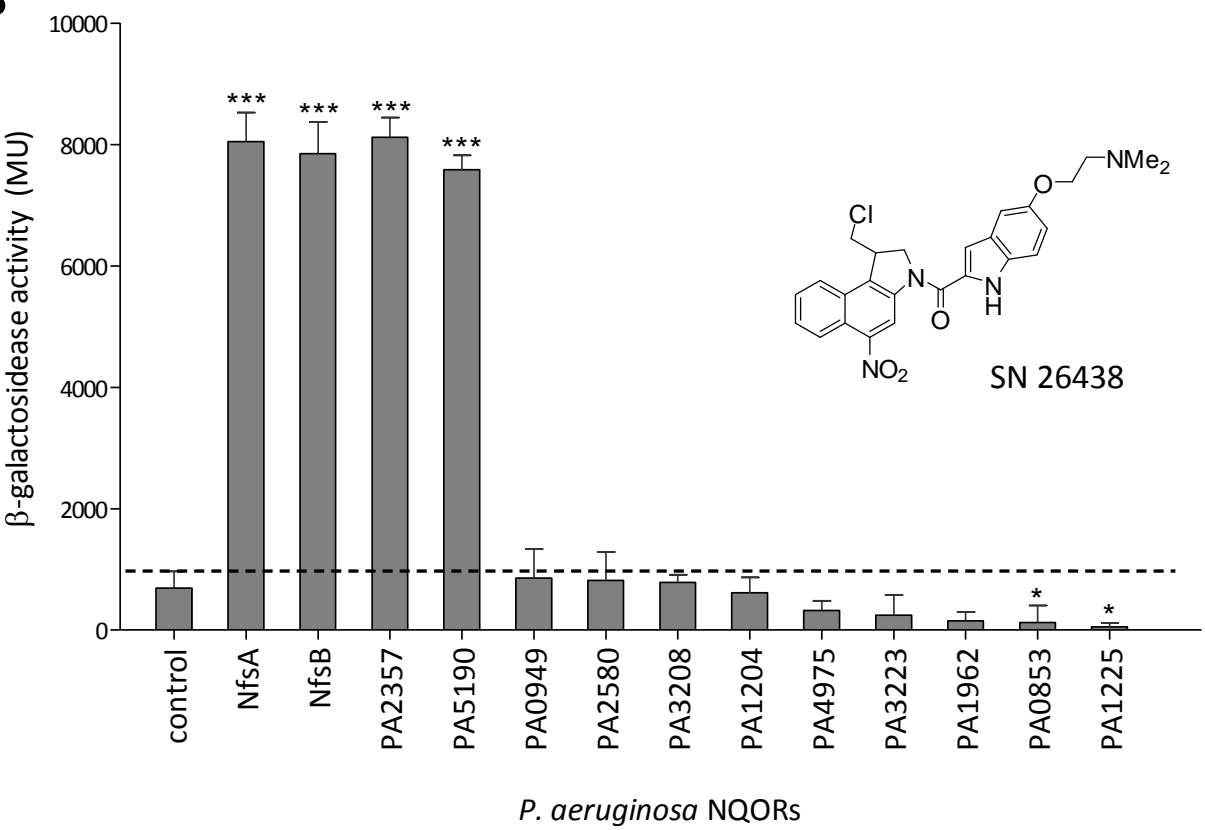

Figure 5.4. CB1954- and SN 26438-induced SOS responses from NQOR overexpression strains of SOS-R2

NQOR over-expressing strains of SOS-R2 were challenged with (A) $20 \mu \mathrm{M}$ CB1954 or (B) $5 \mu \mathrm{M}$ SN 26438 and levels of SOS induction ( $\beta$-galactosidase Miller Units) were measured as described in section 2.18. Data is the mean of three independent experiments each carried out in duplicate and error bars are \pm 1 standard deviation. A black dotted line represents the SOS activity of the empty vector control. 
5.3.2. In vitro kinetics of purified NQOR candidates with CB1954 and SN 26438

Candidate NQORs were cloned into $\mathrm{pET} 28 \mathrm{a}^{+}$vector, expressed as $\mathrm{N}$-terminal $\mathrm{His}_{6}$ tagged proteins in E. coli strain BL21, and purified by nickel affinity chromatography (as described in section 2.8). Apparent steady-state kinetic parameters were measured with CB1954 as substrate as previously reported (139), following the appearance of reduced hydroxylamine metabolites spectrophotometrically at $420 \mathrm{~nm}$ where both the 2- and 4-hydroxylamine end-products have equal absorbance $\left(\varepsilon=1200 \mathrm{M}^{-1} \mathrm{~cm}^{-1}\right.$ (323)). Unfortunately it was not possible to derive any meaningful kinetic data for SN 26438 due to insufficient solubility in aqueous solution.

Consistent with the SOS data, the only $P$. aeruginosa NQOR to exhibit measurable activity with CB1954 in these biochemical assays was PA5190. As a point of comparison the kinetic parameters were also calculated for $\mathrm{Nfs} B$, and it was shown that the E. coli enzyme was almost two orders of magnitude more efficient (in terms of specificity constant; $k_{\text {cat }} / K_{m}$ ) with CB1954 as substrate than its $P$. aeruginosa orthologue (Table 5.2).

Table 5.2. Steady-state kinetics of purified enzymes for CB1954 reduction

\begin{tabular}{llll}
\hline Enzyme & $\boldsymbol{K}_{\boldsymbol{m}}(\boldsymbol{\mu M})^{\mathrm{a}}$ & $\boldsymbol{k}_{\text {cat }}\left(\mathbf{s}^{-1}\right)^{\mathrm{a}}$ & $\boldsymbol{k}_{\text {cat }} / \boldsymbol{K}_{\boldsymbol{m}}\left(\mathbf{M}^{-1} \mathbf{s}^{-1}\right)$ \\
\hline NfsB & $3600 \pm 1100$ & $26 \pm 5$ & 7,200 \\
PA5190 & $580 \pm 210$ & $0.057 \pm 0.006$ & 98 \\
PA2357 & N.D. & N.D. & N.D.
\end{tabular}

\footnotetext{
${ }^{a}$ Apparent steady state kinetic parameters of the corresponding purified recombinant (His6-tagged) enzymes for CB1954 reduction. Data was derived from three individual experiments, and errors are \pm 1 standard deviation.

${ }^{\mathrm{b}}$ No activity detectable by spectroscopic methods.
}

\subsubsection{Sensitisation of the human colon carcinoma cell line HCT-116 to CB1954 and SN 26438 using the in vivo cell culture $\mathrm{IC}_{50}$ assay}

To evaluate the relative efficacy of the $P$. aeruginosa NQORs to sensitizing human tumour cells to SN 26438 and CB1954, we first sought to create human colon carcinoma (HCT-116) cell lines stably transfected with genes encoding these enzymes. However, previous experiences in transfecting HCT-116 have indicated that only a 
subset of NQORs would likely be stably expressed as shown in Figure 5.5 (Gareth Prosser, personal communication and $(139,313))$. As an alternative means to test cytotoxicity of activated prodrug towards human cells, a bacteria delivered enzyme prodrug therapy (BDEPT) assay was developed, using E. coli as a vector; such that NQORs would be stably maintained and expressed by $E$. coli, which would also provide a source of reduced $\mathrm{NAD}(\mathrm{P}) \mathrm{H}$ cofactor, but allow activated prodrug to diffuse out and kill human cells in co-culture.

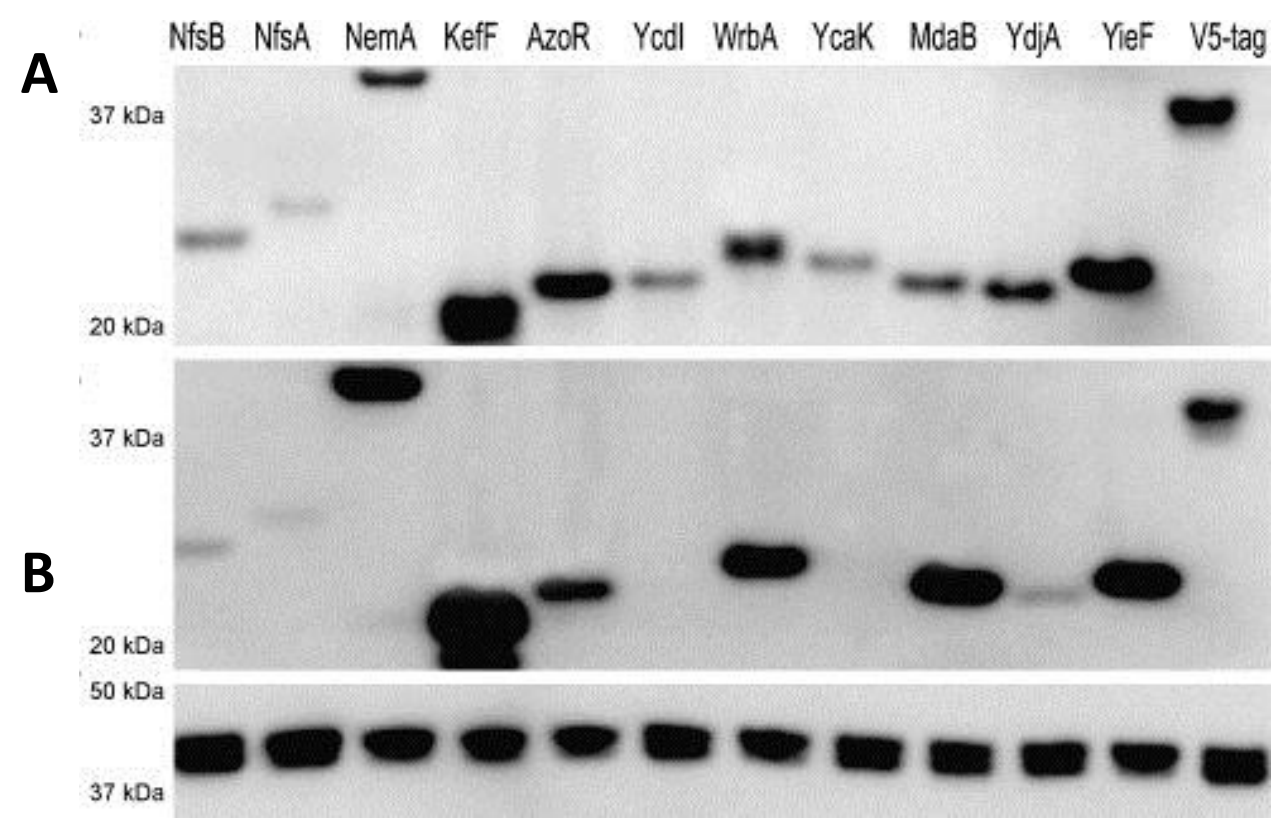

Figure 5.5. Expression of $E$. coli NTRs in the human colon carcinoma cell line HCT-116 as detected by $\mathrm{V} 5$ readthrough western blotting

(A) HCT-116 cells were transiently transfected with F527-V5 vectors containing each of the E. coli NTRs fused to a C-terminal V5 epitope downstream of an amber stop codon. Cells were concurrently infected with Tag-On-Demand ${ }^{\mathrm{TM}}$ Suppressor Supernatant (which enables transient readthrough of the amber stop codon and hence V5 epitope expression), lysed, and immunoblotted for induced C-terminal V5 tag. A 40 kDa V5 tag positive control is included in the right hand lane. (B) HCT-116 cells engineered for stable expression of each of the $E$. coli NQOR candidates were infected, lysed, immunoblotted and quantified as in (A). Note that although all NQORs were expressed transiently to some degree, Ycdl and YcaK were not detectable in stably transfected cell lines. (C) Representative $\beta$-actin loading control (in this instance derived from the transiently transfected immunoblot in (A)). This figure was reproduced from Dr Gareth Prosser's Victoria University of Wellington PhD thesis with permission by Dr David Ackerley (322).

The BDEPT cytotoxicity model towards human cancer cell lines was modified from a previously described protocol wherein purified NQOR proteins were applied to cell culture in media supplemented with prodrug, NADPH and FMN cofactor (138). Rather than applying purified enzymes, we sought to examine the relative potency of candidate NQORs for sensitising HCT-116 human carcinoma cell lines through direct 
application of NQOR-expressing E. coli to the human cells. This adaptation was necessary to overcome (i) difficult-to-purify proteins (313), (ii) unstably expressed NQORs in transfected human cell lines, and (iii) prodrug solubility limitations.

In short, overnight cultures of NQOR over-expressing strains of $E$. coli were initially prepared as for the SOS assays (section 2.18). The day cultures of E. coli and prodrug titrations were, however, prepared in complete cell culture media (CMEM) (without antibiotics, section 2.11) rather than LB amended with ampicillin, IPTG and glucose as for the SOS assays. Mixtures of E. coli were mixed 1:1 with 2-fold dilutions of CB1954 or SN 26438 in CMEM (final concentration ranges of $25 \mu \mathrm{M}$ to $400 \mu \mathrm{M}$ and $0.06 \mu \mathrm{M}$ to $15 \mu \mathrm{M}$, respectively, together with a no prodrug control), and applied to a monolayer of cultured human carcinoma HCT-116 cells. The mixtures were then incubated for $4 \mathrm{~h}$ at $37{ }^{\circ} \mathrm{C}, 5 \% \mathrm{CO}_{2}$, after which the $\mathrm{HCT}-116$ cells were washed several times to remove all bacteria and prodrug. They were then allowed to recover from prodrug-induced DNA damage for $48 \mathrm{~h}$, at which point cellular respiration was measured by formazan production from metabolised tetrazolium compound at $\mathrm{OD}_{490}$ and $\mathrm{IC}_{50}$ values (the concentration of prodrug required to inhibit growth of HCT-116 to $50 \%$ of unchallenged control levels) were calculated (Figure 5.5).

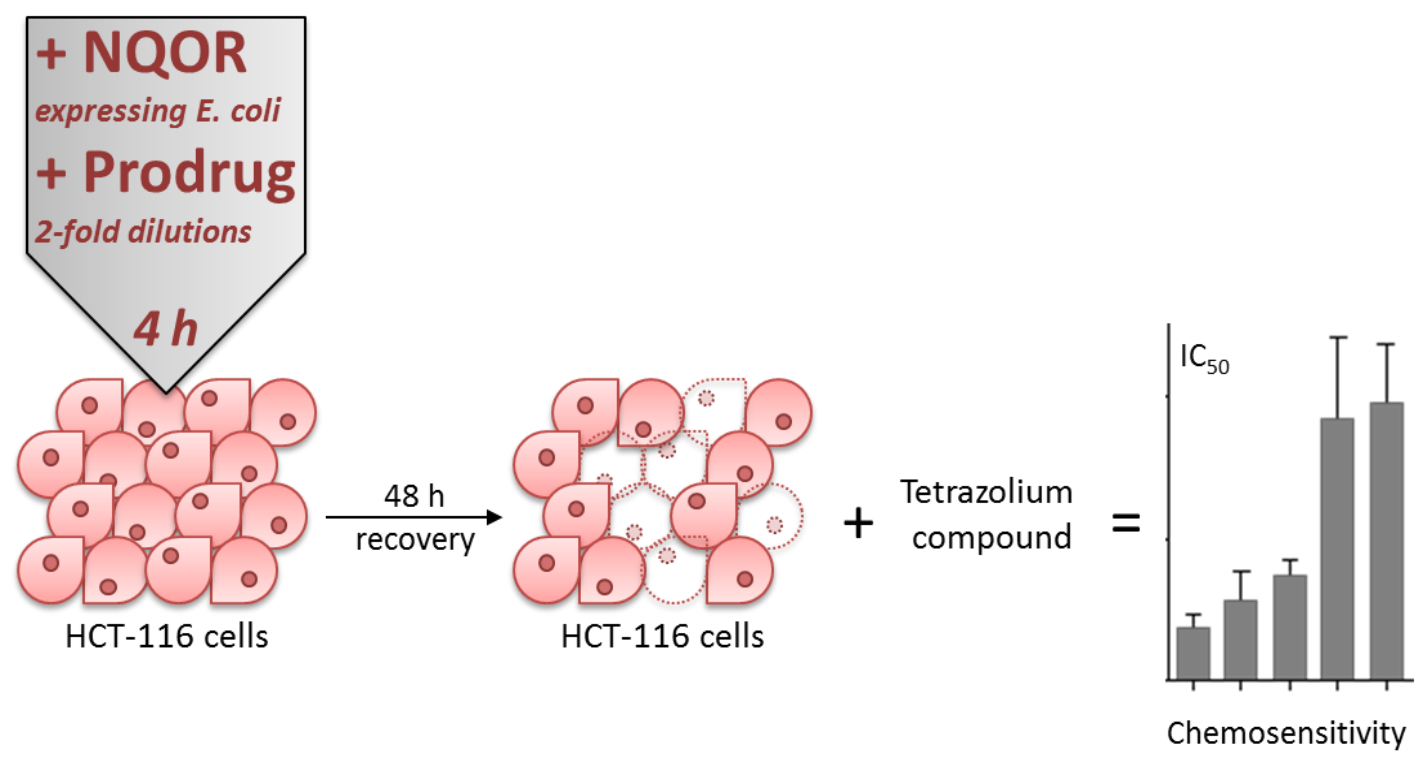

\section{Figure 5.6. Summary of modified BDEPT assay}

The potency of over-expressed NQOR-prodrug partnerships was evaluated by challenging human HCT116 cells for $4 \mathrm{~h}$ with a 1:1 mixture of NQOR-expressing E. coli and 2-fold dilutions of prodrug at $37^{\circ} \mathrm{C}$, $5 \% \mathrm{CO}_{2}$. After washing to remove residual bacteria and prodrug, the HCT-116 cells were allowed to recover from prodrug-induced DNA damage for $48 \mathrm{~h}$ at $37{ }^{\circ} \mathrm{C}, 5 \% \mathrm{CO}_{2}$. Irreversible DNA damage generated by the NQOR-activated prodrug was measured by the cell population's inability to metabolise the tetrazolium compound and enabled calculation of the $\mathrm{IC}_{50}$ for that NQOR-prodrug combination. 
Surprisingly, PA2357 was nearly as effective as NfsB at promoting CB1954mediated killing of the HCT-116 cells ( $\mathrm{IC}_{50}$ of $74.95 \mu \mathrm{M}$ vs. $57 \mu \mathrm{M}$, respectively; Figure 5.6A) even though it did not evoke an E. coli SOS response to CB1954 challenge and purified PA2357 enzyme was not seen to metabolise CB1954 at detectable levels in vitro. PA3223 (AzoR orthologue), PA2580 (MdaB orthologue), and PA2932 (NemA orthologue) enzymes also promoted intermediate levels of killing of HCT-116 cells (IC 50 of $185 \mu \mathrm{M}, 196 \mu \mathrm{M}$, and $212 \mu \mathrm{M}$ respectively, vs. $436 \mu \mathrm{M}$ for the empty plasmid control strain) which had not previously been demonstrated with the SOS or protein assays. However, no $P$. aeruginosa NQORs exhibited improved activity over E. coli NfsB or NfsA in this BDEPT model.

The results of the BDEPT assays with SN 26438 as substrate were more consistent with the previous SOS assays. The $P$. aeruginosa NQORs PA2357 and PA5190 were able to metabolise the SN 26438 prodrug and exhibited significantly improved prodrug-mediated killing of the HCT-116 cells, yielding $\mathrm{IC}_{50}$ values of 0.65 and $0.13 \mu \mathrm{M}$ respectively (relative to $7.8 \mu \mathrm{M}$ for the empty plasmid control). Furthermore, PA5190 showed significantly improved activity $(p<0.005)$ over NfsB in the BDEPT assay (Figure 5.6B). One technical point of difference from the CB1954 BDEPT was that SN 26438 BDEPT was assessed using the NQOR-expression E. coli strain $\triangle 6 K O$, not SOSR-2. The $\triangle 6 K O$ strain, like SOSR-2 lacks the native NQORs $n f s A$ and $n f s B$ but also azoR, yieF, $y c a K$, and $m d a B$; perhaps more importantly, the $\triangle 6 K O$ still possesses an intact tolC outer membrane pore gene. When the BDEPT assay was performed for SN 26438 using SOS-R2 cells, little difference in cytotoxicity was observed for the different NQORexpressing strains, perhaps indicating that TolC plays an important role in efflux of the activated metabolite of SN 26438 from the bacterial cell (a factor that would have less impact on relative $E$. coli SOS measurements than in the BDEPT assay, which mandates a bystander effect to transfer the activated metabolite from the bacterial to the human tumour cells). 


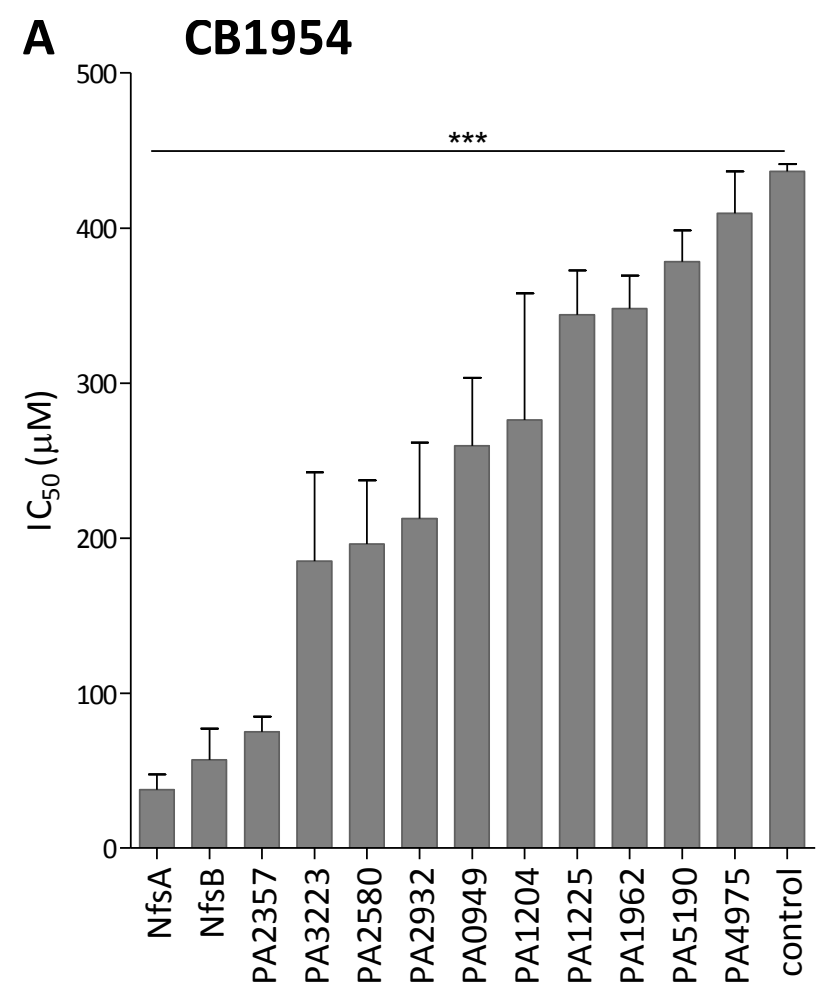

Expressed Enzyme

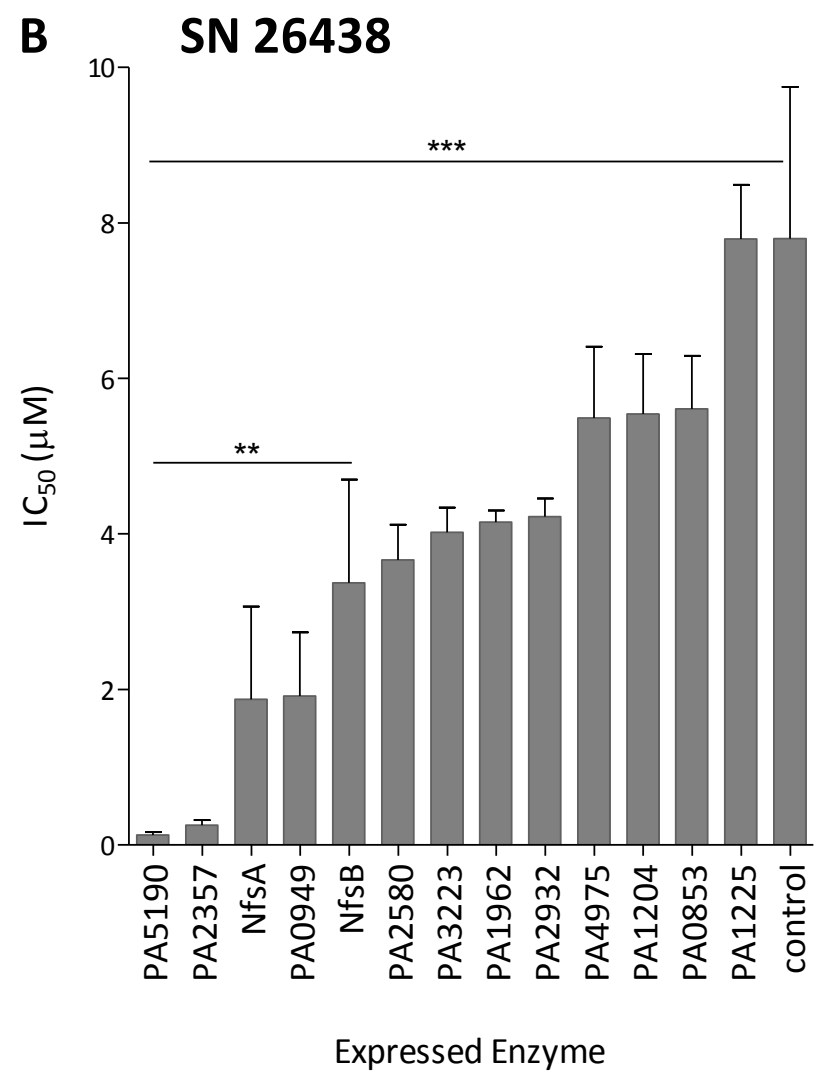

Figure 5.7. HCT-116 human carcinoma BDEPT model assay

HCT-116 colorectal carcinoma cells were challenged with 1:1 mixtures of (A) SOSR-2 NQOR overexpressing strains mixed with $25 \mu \mathrm{M}-400 \mu \mathrm{M}$ CB1954, or (B) $\triangle 6 K O$ strains with $0.06 \mu \mathrm{M}-15 \mu \mathrm{M}$ SN 26438. HCT-116 cells were challenged for $4 \mathrm{~h}$ and then recovered for $48 \mathrm{~h}$ before application of CellTiter $96{ }^{\circledR} \mathrm{AQ}_{\text {ueous }}$ One Solution (Promega) as described in section 2.19. Data is the mean of three independent experiments each carried out in duplicate and error bars are \pm 1 standard deviation. 


\subsubsection{Determination of the $P$. aeruginosa candidate PA5190 bystander effect using three dimensional cell culture assays}

Both NfsB and PA5190 had previously been cloned into the Gateway ${ }^{\mathrm{TM}}$ compatible expression plasmid F527-V5 ${ }^{\text {puro }}$ and transfected into HCT-116 cells. Although previous studies have indicated that, for unknown reasons, only a minority of bacterial nitroreductases are retained and expressed stably using this system, successful stable transfection for both $\mathrm{NfsB}$ and PA5190 was confirmed by concurrent cell infection with TAG stop suppressor adenovirus (Tag-On-Demand ${ }^{\text {TM }}$, Invitrogen) which produced an immunoreactive V5 epitope at the C-terminus of TAG-terminated NQORs detectable by Western Blot (318). Densitometry of the V5 bands in each lane indicated that PA5190 was expressed at approximately 1.4 times the level of NfsB in these cells (318). As we were fortunate to have obtained confirmed stable cell lines expressing NfsB and PA5190, these two NQORs were carried forward to be examined using direct in vitro $\mathrm{IC}_{50}$ and $3 \mathrm{D}$ cell culture (bystander effect) assays. These studies were performed by $\mathrm{Dr}$ Sophie Syddall and our collaborators at the Auckland Cancer Society Research Centre, University of Auckland.

The sensitivity of each of the transfected HCT-116 cell lines towards SN 26438 and CB1954 was evaluated using an in vitro proliferation assay as described by Wilson et al (324). Briefly, replicate monolayers of NfsB, PA5190 or non-transfected HCT-116 cells were evaluated for cell proliferation 5 days post 4-18 $\mathrm{h}$ exposure to a range of concentrations of either CB1954 or SN 26438. The IC $\mathrm{I}_{50}$ of each cell line was determined as the concentration of prodrug required to inhibit cell growth by $50 \%$ relative to untreated controls and was reported as the mean value of two independent experiments (Table 5.3).

Consistent with the results of the BDEPT assay, transfected HCT-116 cell lines over-expressing PA5190 exhibited a greater than 50-fold sensitivity to SN 26438 compared to wild-type controls (Table 5.3). PA5190 also conferred a $>6$-fold increased sensitivity relative to $\mathrm{NfsB}$, again reflecting the trend seen in the BDEPT assay. The results of CB1954 challenge were likewise consistent with the earlier assays in that the NfsB expressing cells were $>7$-fold more sensitive than wild-type controls, but PA5190 was relatively inactive. 
Table 5.3. Fold decrease in IC ${ }_{50}$ of transfected HCT-116 cells $^{*+}$

\begin{tabular}{lll}
\hline Compound & PA5190 & NfsB \\
\hline SN 26438 & $>50$ & 8 \\
CB1954 & 2 & 3000
\end{tabular}

${ }^{*} \mathrm{IC}_{50}$ is reported as the fold decrease in $\mathrm{IC}_{50}$ for transfected cell lines relative to wild-type HCT-116 and NQOR- expressing HCT-116

'This data was reproduced with permission from Dr Sophie Syddall's University of Auckland PhD thesis (318).

The PA5190-SN 26438 partnership was subsequently analysed for ability to generate a bystander effect and thereby kill neighbouring non-transfected cells (Figure 5.1). This was investigated through use of a mixed 3D cell culture model as described by Wilson et al (324). In short, multicellular layers (MCL) of HCT-116 cells were grown on collagen-coated inserts before being challenged with SN 26438. The MCLs were initially seeded with a randomly-distributed mixture of cells which were $10 \%$ NQORexpressing (activators) and 90\% wild type HCT-116 (targets). To determine the bystander effect efficiency (BEE) post-challenge, MCLs were disrupted into a single cell suspension, counted, and then plated in duplicate on to petri dishes to enumerate survival of activators and targets. Determination of clonogenic survival was achieved by treating one of the two petri-dish replicates with puromycin (which the NQORtransfected cells are resistant to, owing to the presence of a puromycin resistance cassette in the $\mathrm{F} 527-\mathrm{V} 5^{\text {puro }}$ plasmid backbone, while the target cells are sensitive). The difference in surviving cell numbers between puromycin treated and untreated cells were used to calculate the clonogenic survival of activators and targets, and therefore the BEE of SN 26438 in tandem with either NfSB or PA5190.

Surprisingly there was a large difference between the bystander activity demonstrated with NfsB-dependent activation of SN 26438 and PA5190-dependent activation (Figure 5.7). Whereas the minority population of PA5190 activator cells was able to almost completely eradicate the target $\mathrm{HCT}-116$ population, the NfsB activators did not appear to exhibit a particularly strong bystander effect (an exact value for the NfsB BEE was unable to be calculated as the co-culture cell killing did not fall below $10 \%$, precluding the $\mathrm{C} 10$ from being estimated accurately). It should be noted that despite both experiments being seeded with $10 \%$ activator cells, a higher population of 
activator cells was ultimately found to be present in the PA5190 co-culture (9\%) relative to the NfsB co-culture (3.7\%). However, the difference in bystander effect cannot be attributed solely to this, as in a preliminary experiment a very high BEE value (84\%) was also achieved for the PA5190 cells when only $2.6 \%$ activator cells were present (results not shown).

A

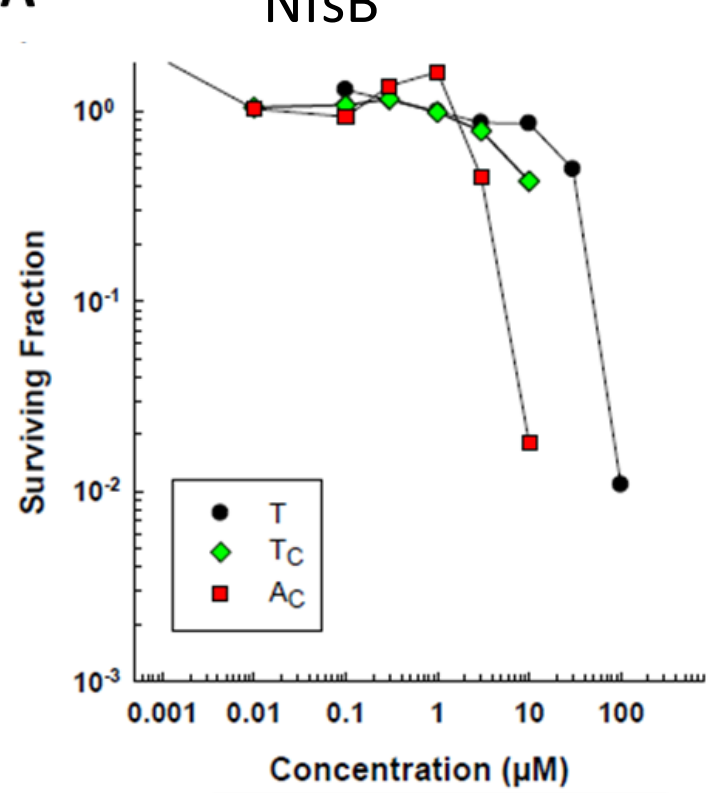

B

\begin{tabular}{|l|c|}
\hline Compound & SN26438 \\
\hline \hline Actual \%NQOR & 3.7 \\
\hline BEE $(\%)$ & N.D. \\
\hline$T(\mu M)$ & 63 \\
\hline$T_{C}(\mu M)$ & $>10$ \\
\hline$A_{C}(\mu M)$ & 5.5 \\
\hline
\end{tabular}

PA5109

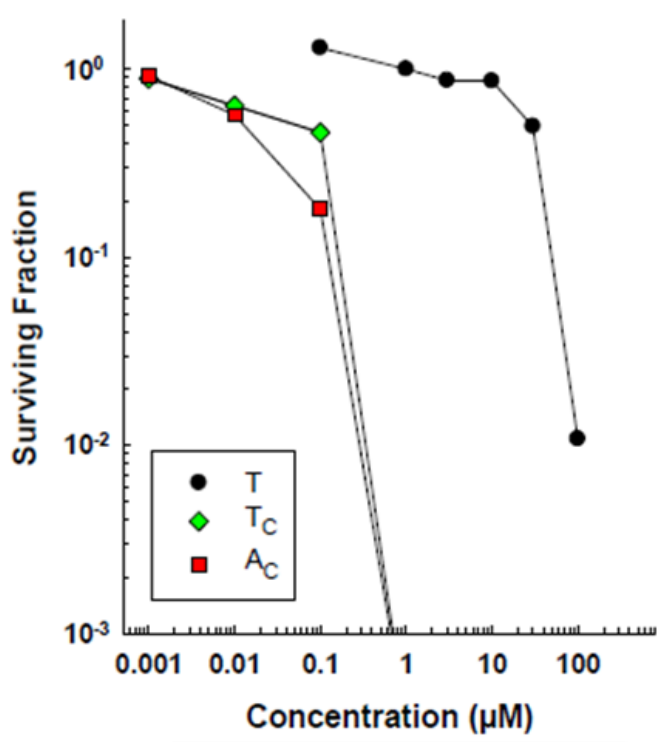

\begin{tabular}{|l|c|}
\hline Compound & SN26438 \\
\hline \hline Actual \%NQOR & 9 \\
\hline BEE $(\%)$ & 94 \\
\hline$T^{T}(\mu \mathrm{M})$ & 63 \\
\hline$T_{C}(\mu M)$ & 0.17 \\
\hline$A_{C}(\mu M)$ & 0.12 \\
\hline
\end{tabular}

Figure 5.8. Enhanced bystander efficiency of SN 26438 was demonstrated with PA5190-dependent activation compared to NfsB

(A) Representative bystander efficiency graphs of NfsB-activation of SN 26438. Values are from one $\mathrm{MCL}$ experiment. The prodrug concentration for $10 \%$ survival (C10) of target cells (wild-type HCT-116) grown without activators (T), targets in co-culture (Tc) and 100\% activators (NQOR-expressing cells) in coculture (Ac) were determined by interpolation. (B) IC $\mathrm{C}_{50}$ and $\mathrm{MCL}$ data from SN 26438 activation. As per Table 5.3 the $\mathrm{IC}_{50}$ is the concentration of prodrug required to inhibit growth by $50 \%$ of untreated controls following $18 \mathrm{~h}$ drug exposure and regrowth for 5 days. BEE bystander effect efficiency calculates the drug bystander effect: ((LogC10T-LogC10TC)/(LogC10T-LogC10AC)). The T/AC ratio is the ratio of the average target $\mathrm{C} 10$ divided by the activator $\mathrm{C} 10$. This data was reproduced with permission from Dr Sophie Syddall's University of Auckland PhD thesis (318). 


\subsection{Discussion}

The primary objective of this work was to identify novel enzymes for GDEPT with greater efficacy than the "gold standard" E. coli enzyme NfsB, which under clinical evaluation with CB1954 was found to have a $K_{m}$ ca. 100-fold higher than the concentration achievable in patient serum at the maximum tolerated dose $(8,9)$. Although PA5190, the most active of the purified $P$. aeruginosa enzymes, did possess a lower apparent $K_{m}$ for CB1954 than NfsB as measured at $250 \mu \mathrm{M} N A D H$, it also had a very low apparent $k_{c a t}$, and was nearly two orders of magnitude less efficient than NfsB in terms of $k_{\text {cat }} / K_{m}$. This low level of activity at a purified enzyme level was mirrored by only low levels of SOS induction observed in SOS-R2 when the PA5190 over-expressing strain was challenged with $20 \mu \mathrm{M}$ CB1954, and by an inability to substantially sensitise HCT-116 cells to this prodrug. In contrast, PA5190 metabolism of the lead nitro-CBI prodrug candidate SN 26438 consistently yielded high levels of activity in all assay systems tested.

Our laboratory has previously demonstrated the effectiveness of the $\beta$ galactosidase based-SOS assay for rapid evaluation of novel nitroreductase candidates for GDEPT $(139,312)$. As an additional method for evaluating the ability of $P$. aeruginosa-derived NQORs to bioreductively activate CB1954 and SN 26438 prodrugs, we developed a BDEPT model to evaluate killing of human tumour cells by different NQOR over-expressing E. coli strains. This model appears to be able to detect low levels of activation of CB1954 and SN 26438 that could not be detected in the SOS assay or by spectrophotometric analysis of purified enzyme activities. Most significantly, exposure of HCT-116 cells to E. coli over-expressing PA5190 or PA2357 resulted in $>26$ fold and $>13$ fold heightened sensitivity to SN 26438, respectively, relative to E. coli over-expressing NfsB. PA2357 also conferred substantially heightened sensitivity to CB1954 in this model, which was unexpected given that this enzyme had not appeared to metabolise CB1954 in either E. coli SOS or purified protein assays. It is unclear whether recombinant $\mathrm{His}_{6}$ tagged PA2357 enzyme might have been inactive in its purified form, therefore limiting kinetic assessment, or if overexpression of PA2357 might have sensitised HCT-116 cells to CB1954 by some indirect process. Irrespective, as even the BDEPT assay did not indicate any of the $P$. aeruginosa NQORs to be superior to either NfsA or NfsB from E. coli in terms of 
CB1954 metabolism, this anomaly was not pursued further. In the BDEPT assays, SN 26438 was also more toxic to HCT-116 than CB1954. While this heightened toxicity is a very attractive feature, what is more important is the therapeutic index of the drug, which will derive from the maximum tolerated dose that can be administered to patients and the maximum concentration that can then be achieved at tumour cells. To the best of our knowledge these measurements have not yet been made in humans using SN 26438. Thus, exactly how promising SN26438 might prove for next generation clinical GDEPT therapies remains to be determined.

Despite the unexplained result with PA2357 and CB1954, we believe that the BDEPT assay is a very useful tool for evaluating enzyme-prodrug combinations for their potential use in GDEPT by circumventing experimental difficulties with protein purification and stable transfection of human cell lines. It may also provide greater sensitivity than the SOS assay in its ability to indicate intermediate levels of prodrug metabolism, however, rigorous evaluation is still required to determine whether this is truly the case. Nonetheless, the BDEPT assay is certainly capable of generating highly reproducible results; but one caveat is that day-to-day values appeared to be directly proportional to the "age" of the HCT-116 cell line, although variations between sameday replicates were low. Because of this, experimental replicates are recommended to be completed with HCT-116 cells lines within 1-3 passages of one another.

Whereas none of the $P$. aeruginosa NQORs were superior to $E$. coli $\mathrm{Nfs} A$ or NfsB in terms of CB1954 metabolism, the BDEPT assay indicated that PA5190 was substantially more active than these enzymes with SN 26438, and this enzyme prodrug combination was taken forward for further analysis alongside NfsB in stably transfected HCT-116 cell lines. Further indication of PA5190 as a superior enzyme for SN 26438 GDEPT applications was evident in multi-cell layered cultures when PA5190 was found to mediate the strongest bystander effect hitherto seen in this system ( $\mathrm{Dr}$ Adam Patterson, personal communication). In contrast, NfsB bystander activity was insufficiently strong to enable a BEE value to be calculated, as the co-culture cell killing did not fall below $10 \%$. This is one of the first experiments to compare two different enzymes for bystander activity with the same prodrug, and to our knowledge, the first time that this has led to profoundly different bystander outcomes. It is possible that PA5190 may metabolise SN 26438 to a different end-metabolite but further 
investigation of this would require liquid chromatography-mass spectrophotometry to define the products generated by each enzyme.

Importantly, the disparity between PA5190 and NfsB cell killing due to SN 26438 activation in the in vitro IC 50 proliferation assay and in the 3D multi-cell layers highlights the importance of evaluating potential GDEPT enzyme-prodrug partnerships in a 3D bystander model, as low cell density may dramatically underestimate cytotoxic potential. We surmise that the cytotoxic potential of high-bystander prodrugs may be dramatically underestimated in low cell density proliferation assays due to washout and loss in the media.

SN 26438 may exhibit a uniquely strong bystander effect due to its lipophilicity and also its target specificity. Reduced nitro-CBI very selectively binds adenine in the minor groove of DNA (Figure 5.1BC; $(317,318)$ ); whereas CB1954-derived DNA lesions appear to result from non-selective crosslinking $(141,142)$. The lipophilicity and target specificity of SN 26438 may contribute to the enhanced bystander efficiency relative to CB1954 (314) in that (i) its reduced metabolites are more easily transferred through lipid membrane of neighbouring cells, and that (ii) fewer targets exist per cell, which may enable the activated SN 26438 metabolite to diffuse through more cells before ultimately reacting with DNA. Further experimentation will be required to determine if this result is unique to SN 26438, and also to evaluate the potential of the PA5190 / SN 26438 combination for GDEPT in in vivo models.

In other work we have shown that the SOS assay can not only be used for enzyme evaluation, but also to improve the catalytic activation of genotoxic prodrug substrates by directed evolution (in this case, a process of random mutation applied at a single gene level followed by cloning of gene variants into a plasmid vector and screening for improved activity in a suitable bacterial reporter strain; (313)). Using the SOS assay along with targeted mutagenesis of PA5190, there is potential to further improve SN 26438 metabolism by generating PA5190 variants with enhanced catalytic efficiency. This could possibly prove effective in enhancing the potency of the PA5190 / SN 26438 paradigm for GDEPT. 


\section{Chapter 6: Summary, conclusions, and future directions}

\subsection{Research Motivation and Summary}

$P$. aeruginosa is a genetically flexible and innately drug-resistant pathogen (2). The ability of this organism to adapt and defend itself in the face of a complex and constantly changing host immune response make $P$. aeruginosa a major contributor to increased morbidity and mortality rates from nosocomial infections (3). As such, there is much interest in characterising the $P$. aeruginosa defence mechanisms that allow the organism to persist and establish disease. As a one of the first major antibacterial assaults by the immune systems includes a concentrated release of $\mathrm{H}_{2} \mathrm{O}_{2}$, oxidative stress tolerance by $P$. aeruginosa is an area of intense research interest.

Soluble quinone oxidoreductases have been identified in mammals as being involved in oxidative stress protection; and although NQORs have been found to be widely distributed throughout bacterial species (119), few bacterial NQORs have been shown to definitively confer a protective phenotype under oxidative stress. One bacterium where such a phenotype was observed, however, was in another species of Pseudomonas, P. putida. Based on this previous work, the research described in this thesis initially aimed to characterise an in vitro biological role for $\mathrm{H}_{2} \mathrm{O}_{2}$ protection by NQORs in $P$. aeruginosa.

Additionally, and in appreciation of the complex interaction between the host and pathogen in disease establishment, an in vivo cell culture infection model was developed. This sought to investigate the role of $P$. aeruginosa NQORs in a more multifaceted scenario whereby the bacteria would not only be exposed to the $\mathrm{H}_{2} \mathrm{O}_{2}$ but also to other naturally released immune effector signals by murine macrophages. We also used this method to recover $P$. aeruginosa RNA from viable bacteria located within the macrophage, a technical accomplishment that has not previously been reported. In doing so, we were able to address the potential of NQORs at the level of a global transcriptional response.

In a separate but related aspect of NQOR activity, the simultaneous two electron reductive mechanism of $P$. aeruginosa NQORs was explored in the therapeutic application of GDEPT. A very attractive emerging focus in the field is the use of oxygenindependent (i.e. divalent) reductase enzymes to leverage the wealth of compounds 
that have been developed to target tumour hypoxia (146), and thereby expand their utility to aerobic tumour tissues also. Whereas the 'gold standard' enzyme E. coli NfsB has been found to have a very high $k_{\text {cat }}$ with nitro-aromatic prodrugs in general, it also tends to have a very high (millimolar) $\mathrm{K}_{\mathrm{m}}$, and this is likely to limit its clinical utility in GDEPT, with most plausible prodrug partners only able to achieve micromolar concentrations in patient serum $(138,139,146,318,322)$. In an effort to improve on this we tested the ability of the $P$. aeruginosa NQORs to activate the lead nitro-CBI prodrug candidate SN 26438, and found that the $P$. aeruginosa NfsB orthologue (PA5190) was greatly superior to $E$. coli NfsB in terms of its ability to generate cytotoxic damage and a high bystander effect; both very important features for GDEPT.

\subsection{Key Findings}

\subsubsection{Potential functional redundancy of NQORS in $P$. aeruginosa}

In an early funding application for this study it was hypothesised that $P$. aeruginosa would possess two soluble quinone oxidoreductase enzymes with antioxidant potential, based on homology searches and the general state of knowledge at the time. Instead, we ultimately identified 10 unique genes with $>25 \%$ amino acid identity to quinone oxidoreductases that have been proposed to play such a role in other bacteria. Using a select subset of NQORs with either (i) shared identity with $P$. putida

ChrR; (ii) shared identity with mammalian DT-diaphorase; or (iii) shared identity with $E$. coli WrbA, MdaB, or NfsB, we found that gene-deletion or over-expression of a single NQOR candidate was unlikely to yield a measurable $\mathrm{H}_{2} \mathrm{O}_{2}$ tolerance phenotype in $P$. aeruginosa due to a masking effect from the potent KatA catalase. Moreover, comparison of NQOR over-expression phenotypes in a katA-null $P$. aeruginosa strain with the effect of over-expressing the same NQORs in a $\Delta c h r R$ strain of $P$. putida indicated that functional redundancy of the numerous endogenous $P$. aeruginosa NQORs might also mask the contribution of any individual NQOR candidate to oxidative stress resistance. If nothing else, these findings collectively indicate that our original goal of finding inhibitors to individual NQORs as a means of combating the beneficial effect of these hypothesised virulence factors was probably misguided, and 
for numerous reasons specific NQOR inhibitors would be unlikely to provide any therapeutic benefit in combatting $P$. aeruginosa infections.

\subsubsection{Development of an in vivo cell culture infection model for the isolation of RNA from viable, macrophage-engulfed $P$. aeruginosa}

We next reported development of an in vivo cell culture infection model whereby viable, macrophage-engulfed $P$. aeruginosa are recovered for analysis. Recovering $P$. aeruginosa RNA from viable bacteria located within the macrophage is a technical accomplishment that has not previously been reported. Additionally, this method demonstrated the potential for evaluating the survival of $P$. aeruginosa strains en masse, using flow cytometry to achieve rapid enumeration.

In one of its first applications the in vivo cell culture model revealed a phenotype for both NQOR gene-deleted and over-expression strains, suggesting that the $\triangle P A 1204$ mutant was more sensitive to macrophage engulfment than wild type PAO1. Given the lack of sensitivity of this mutant strain to $\mathrm{H}_{2} \mathrm{O}_{2}$ challenge in vitro it may be that PA1204 plays some additional role beyond just $\mathrm{H}_{2} \mathrm{O}_{2}$ tolerance in enabling the bacterium to withstand phagocytosis. Other results were more consistent with our observations in vitro, with over-expressed PA2580 and PA0949 both promoting $\mathrm{H}_{2} \mathrm{O}_{2}$ tolerance in vitro (albeit only in the katA-null background) and enhancing survival in the macrophage model. Overall, the results from this aspect of the study strongly support the investigation of $P$. aeruginosa oxidation stress mechanisms in a more complex model rather than an isolated cell system whereby the stressor is a constituent in nutrient rich growth media.

\subsubsection{The transcriptional response of macrophage-engulfed $P$. aeruginosa}

The in vivo cell culture model also enabled microarray analysis of macrophageengulfed $P$. aeruginosa. First, and perhaps most importantly, the transcriptional response of macrophage-engulfed $P$. aeruginosa indicated that the bacteria are, to a degree, turning off or down-regulating mechanisms that are "known" virulence factors. For example, katA catalase, an accepted virulence determinant $(111,325)$ was downregulated in Internal PAO1, as were katB and katE, various DNA repair genes, and 
the putative NQOR PA2580, which we had shown to promote survival in the presence of macrophages. The idea that $P$. aeruginosa intentionally down-regulates "protective" processes is consistent with previous research themes that indicate $P$. aeruginosa may adapt to assault from the immune system by enhancing its own mutation rate to generate variants with enhanced long term survival $(281,283,284,326)$. However, it is also possible to get too much of a bad thing, and it may be that down-regulation of the pyoverdine genes in the macrophage internalised cells was not just a consequence of iron no longer being required to power the (down-regulated) KatA/B/E catalases, but an attempt by the cell to limit iron uptake because of the danger of generating hydroxyl radicals via Fenton reactions.

The transcriptional response also indicated that several potential NQORs were up-regulated in the presence of macrophages. As our primary motivation to do microarray analysis was to facilitate a more targeted investigation of NQOR function, this outcome may facilitate future NQOR characterisation studies.

\subsubsection{PA5190 and SN 26438: A novel enzyme-prodrug pairing for GDEPT}

In a different aspect of this study we explored the potential of $P$. aeruginosa NQORs to activate hypoxia targeting anti-cancer prodrugs, thereby exploiting the simultaneous two electron transfer mechanism noted in Chapter 3. We found that a lead prodrug candidate, SN 26438, became highly toxic upon reduction by the $P$. aeruginosa NQOR PA5190; and that its activation by PA5190 was $>26$ fold more cytotoxic to cultured HCT-116 cells in a BDEPT-like model system than activation by the "gold standard" $E$. coli NfsB. Perhaps the most exciting aspect of this work was the demonstration that PA5190 activation of SN 26438 produced an unprecedented bystander effect, with 94\% transfer of toxicity from a minority of PA5190-expressing cells to the target cell population in a 3D multi-cell layer model. This was one of the first times two different NQORs have been compared for bystander efficiency using the same prodrug. The profoundly different bystander outcomes - whatever the reason for it - further implicates PA5190 as a superior enzyme for GDEPT therapy and suggests that a more thorough investigation into this novel enzyme-prodrug partnership is warranted. 


\subsection{Future Directions}

\subsubsection{Elucidating a biological role for $P$. aeruginosa NQORs}

As noted in section 6.2.1 we believe that there may be functional redundancy between the numerous $P$. aeruginosa NQORs. Although gene-deletion efforts proved troublesome in this study, a next step to elucidating an $\mathrm{H}_{2} \mathrm{O}_{2}$-protective role for NQORs in $P$. aeruginosa could be to generate multiple NQOR deletions in a single (presumably also katA-null) strain. If the bacterium can tolerate a single gene-deletion through functional redundancy, it may be that generating and evaluating different combinations of multi-NQOR knockouts would prove this redundancy. Again, assessing the viability of multiple isogenic deletion strains in parallel would be greatly facilitated by the flow cytometry protocols developed in this work.

Additionally, just because an NQOR deletion does not result in an $\mathrm{H}_{2} \mathrm{O}_{2}$ sensitive growth phenotype it does not mean that other important cellular processes are not being perturbed. Phenotype MicroArrays, developed by Biolog Inc. which can screen over 2000 phenotypes (http://www.biolog.com), are a powerful tool that could aid in understanding the implications of NQOR deletion on a wide range of metabolic processes relevant to $P$. aeruginosa survival. The Biolog Inc. system has the capacity to screen for acquired metabolic and chemical sensitivities under a vast array of culture conditions. Demonstrating the potential of the Phenotype MicroArray, several studies have uncovered new phenotypes using this assay (327-330). Although the Phenotype MicroArray may require a significant financial investment, in the long run, especially with redundant enzyme activities, the "shot-gun" approach may prove to be the most cost-effective and time-effective approach to understanding $P$. aeruginosa NQOR function.

\subsubsection{Implications of research for $\boldsymbol{P}$. aeruginosa therapy}

Intravenous iron treatment has been well documented as increasing the risk of bacterial infection in the clinical setting (331). In $P$. aeruginosa, a known virulence determinant, pyoverdine, aids in sequestering iron from the intercellular iron stores in eukaryotic hosts for the purpose of supporting bacterial growth $(245,246)$; therefore 
the siderophore has been investigated as a potential therapeutic target (332-336). The approaches to siderophore-based therapy encompass (i) iron starvation, (ii) inhibition of siderophore biosynthesis, and (iii) siderophore-mediated drug delivery (336). To date, the only method to show promising antimicrobial activity in animal models is siderophore-mediated drug delivery, whereby a siderophore is used to selectively deliver either a toxic heavy metal or a conjugated cytotoxin to the bacterial cells $(336,337)$.

In our analysis of the transcriptional response of $P$. aeruginosa to phagocytosis we found that 8 of the 11 pyoverdine synthesis genes were uniformly down-regulated. This may suggest that the efforts to combat bacterial infection by iron starvation or inhibition of siderophore biosynthesis are misguided. We speculate that pyoverdine biosynthesis may prove disadvantageous to macrophage-internalised bacteria as the increase in pyoverdine would increase iron levels and therefore increase $\mathrm{H}_{2} \mathrm{O}_{2}$ sensitivity via Fenton chemistry. Although perhaps now moving beyond the bounds of reasonable speculation, it would be interesting indeed if inhalation of iron-loaded pyoverdine as an aerosol were to prove beneficial in combatting $P$. aeruginosa airway infections.

A more reasonable strategy for treating $P$. aeruginosa infection may come from our findings that novel anti-cancer prodrugs can be activated by endogenous $P$. aeruginosa NQORs, in particular PA5190 and PA2357 (MsuE). Findings from our microarray analysis suggest that DNA damage repair mechanisms are being downregulated during infection. We hypothesise that this is to increase hypermutation, however, we may be able to take advantage of $P$. aeruginosa "letting its guard down" by administering DNA damaging prodrugs. For example, both PA5190 and PA2357 were able to metabolise SN 26438 effectively. Obviously the profound bystander effect of the activated drug would not be advantageous for therapy as cytotoxic transfer would also debilitate the host cell and most likely have a negative impact on total bacterial clearance. However, the Ackerley lab has been investigating other nitroaromatic prodrugs that completely lack a bystander effect, which should enable ablation of the bacterial cell without harm to the eukaryote host; and indeed, there are several examples of genotoxic prodrug antibiotics in current use that employ this mechanism (338-341). PA5190 and PA2357 have yet to be tested with these nilbystander prodrugs, but the SOS assay is ideally suited for conducting such an analysis. 
Moreover, according to our microarray data, PA2357 is upregulated at the same time that DNA repair systems are down-regulated in the phagocytosed bacteria, and may therefore prove a particularly suitable target. Better yet, if a nil-bystander prodrug can be identified that is activated by multiple $P$. aeruginosa NQORs, then this would greatly diminish the chances of drug resistance arising during therapy.

\subsection{Concluding Remarks}

In addition to the primary research findings, a number of novel experimental protocols were developed during this study that have already demonstrated relevance to the wider scientific community and have been applied to other research endeavours. The first of these is the rapid flow cytometry protocol for enumeration of viable bacterial cells, which was used to assess the antimicrobial effect of novel fabrics. This data, together with supporting confocal micrographs for qualitative effect, has led to a coauthored manuscript that is currently in preparation (CK Fonseca-Paris, LK Green, JH Johnston, DF Ackerley. Antimicrobial properties of palladium nanoparticles immobilized in and onto wool fibres. Intended for submission to Journal of Biotechnology).

A second co-authored manuscript has arisen from use of the BDEPT model to assess the cytotoxic potential of evolved variants of FRase I (Flavin Reductase I from Vibrio fischeri) in GDEPT, as FRase I, for an unknown reason, was unable to be stably expressed in HCT-116 cells. Application of BDEPT allowed us to determine prodrug activation and cytotoxic phenotypes for an enzyme-prodrug paradigm that was otherwise untestable in our existing models. This work is currently accepted in press in Biochemical Pharmacology (313).

A final example of how this work has already proven relevant to the wider scientific community is the interest our macrophage model has generated. The laboratory of Professor Steffen Thiel (Aarhus University, Denmark) has requested access to our protocol to test the impact of complement deposition on mannanbinding proteins in pathogen recognition. 


\section{Appendix 1: Antimicrobial properties nanoparticle-coated wool fibres}

A collaborative approach with Carla Fonseca-Paris and James Johnston of the Victoria Univeristy of Wellington's School of Chemical and Physical Sciences. Manuscript in preparation for publication.

\section{A1.1. Introduction}

Hospital-acquired infections (HAI) are a major concern for medical providers worldwide as they are a major cause of death and contributor to increased morbidity in patients (342). The financial impact of HAl attributed with extended hospital stays, additional care and infection control was estimated in 2007 to cost approximates USD\$595,000 per individual hospital per annum (343). Particularly problematic are the increasingly drug resistant pathogens like the methicillin-resistant strains of Staphalcoccus aureus, a gram positive bacterium responsible for $>25 \%$ of all nosocomial infections (344). As with S. aureus, many gram negative pathogens like enteropathogenic Escherichia coli are also acquiring antibiotic resistance at elevated rates (345). With increasing rates of resistance, there is currently much commercial interest in developing novel textiles that are able to control the growth of both grampositive and gram-negative bacteria alike (346-349). Incorporating antimicrobial or even microbiocidal material in both hospital bedding and functional wear may have the potential to assist in reducing HAl transmission rates (346).

Silver metal and its compounds have long been known to exhibit a strong toxicity to a wide range of micro-organisms since ancient times. This biocidal property is being used in many commercial products and in biomedical applications like medical implant coatings and wound dressings. Metallic nanoparticles such as silver have increased chemical activity as a result of their large surface to volume ratios and crystallographic surface structures. They also exhibit antibacterial properties due to their strong binding to the electron-donating groups in the surface of the bacterial cells and because of their small size can penetrate into them, resulting in antimicrobial activity (350). This biocidal activity is also exhibited by nanoparticles of gold and palladium. This has promoted research in the activity of gold, palladium and silver nanoparticle-based compounds. 
This section presents and summarises a study were nano-palladium particles immobilised on wool fibres significantly affected the bactericidal qualities of merino wool fibres. It was demonstrated that multifunctional composite materials (metalcoated nanoparticles) could be stably incorporated in to the wool, a natural and environmentally friendly support structure. It was also established that the particle size could be controlled by adjusting the temperature at which heat was applied to wool-palladium solutions for nanoparticle formation and that palladium-loading could be chemically controlled to vary palladium concentration. The bacteria quantification methods in this study were also used a proof of principle that flow cytometry could accurately be used to measure survivability for the Chapter 4 infection model.

\section{A1.2. Summary of findings}

To justify the use of palladium-coated nanoparticles, it was firstly demsonstrated that palladium-loading of merino had increase bacteriocidal qualities over the previously characterised silver hybrid as described by Kelly and Johnston in 2011. Additionally, the effect of nano-palladium concentraion (paritcle size) and particle immobilisation temperature was examined. Antimicrobial testing was performed by washing stationary-phase E. coli W3110 in PBS to remove traces of nutrient media. $50 \mu \mathrm{l}$ of washed bacteria wash then absorbed into $150 \mathrm{mg}$ of wool in triplicate and stored at room temperature protected from light. After 7 days incubation, E. coli was washed free of the woolen plug and examined by CFU, flow cytomentry or confocal microscopy.

The key findings were:

1. Palladium, in comparisoin to silver-based compsites, increased the killing efficiency of merio wool-nanoparticle hybrids by nearly $100 \%$ depending on metal concentration (Figure A2.1).

2. Bacterial viability decreased as palladium concentration increased and composites containing $>65$ ppm Pd-merino (6.50 mg Pd/g-wool) were leathal in all cases (Figure A2.2).

3. Merino-palladium fibres have a greater biocidal properties than crossbredpalladium at $\mathrm{Pd}$ concentrations $>6.5 \mathrm{ppm}$ (Figure A2.3.). 


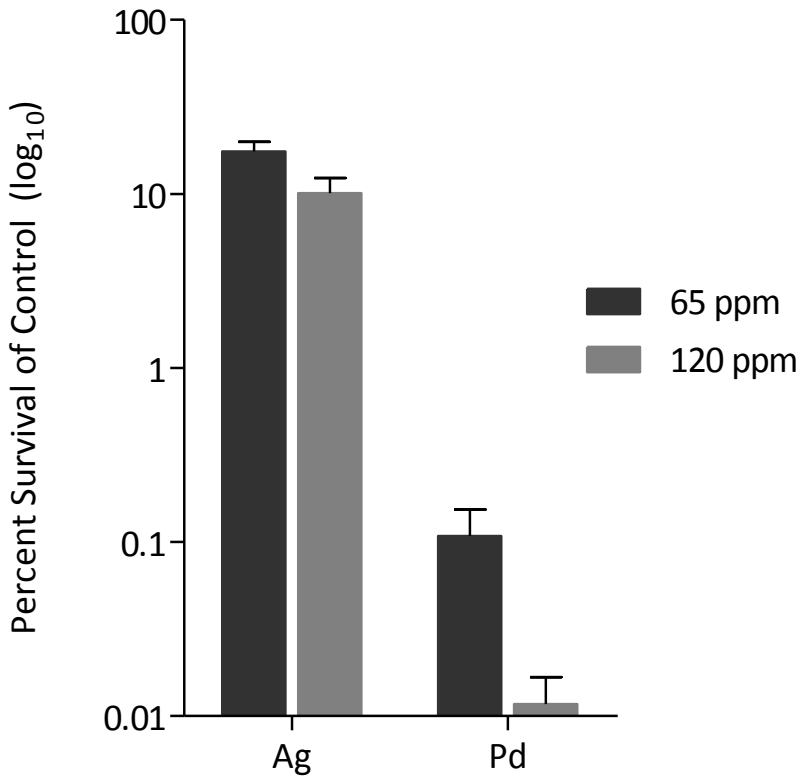

Figure A1.1. E. coli killing ability of silver vs. palladium composites

Nanosilver- and nanopalladium-merino wool composites of $65 \mathrm{ppm}$ and $120 \mathrm{ppm}$ were treated for 7 days with PBS-washed $E$. coli. Bacteria were then quantified by CFU. The data presented is a representative of three experimental replicates and error bars depict \pm 1 standard deviation.

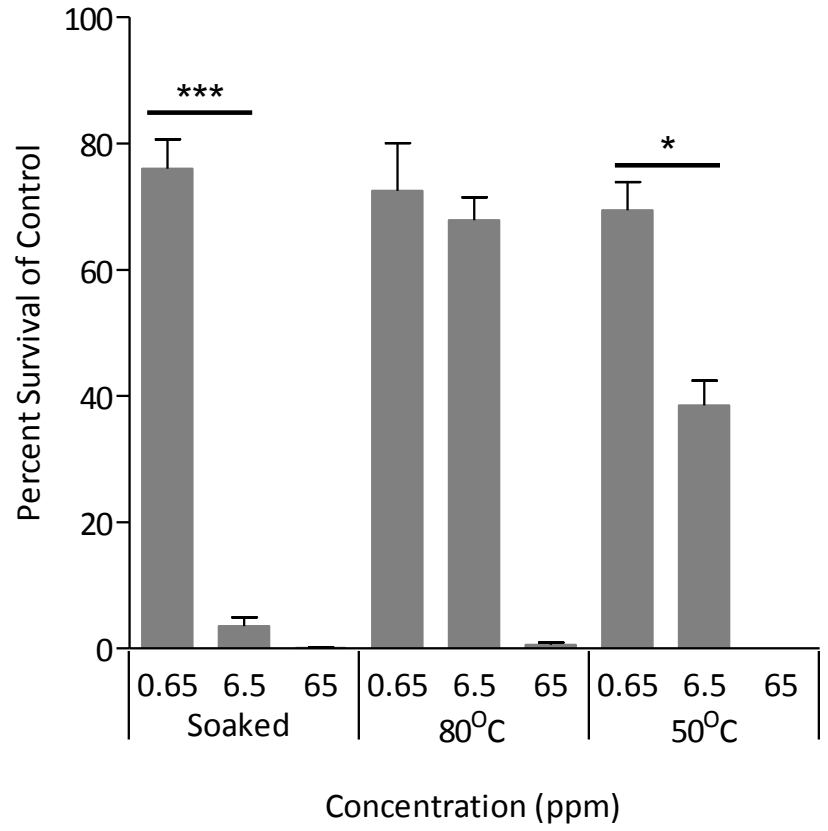

Figure A1.2. Nanopalladium-merino wool composite antimicrobial activity by palladium concentration

Nanopalladium-merino wool composites $(0.65 \mathrm{ppm}, 6.5 \mathrm{ppm}$, and $65 \mathrm{ppm})$ were prepared at room temperature, $80^{\circ} \mathrm{C}$, and $50^{\circ} \mathrm{C}$. The wool composites were treated for 7 days with PBS-washed E. coli. Bacteria were then quantified by CFU. The data presented is a representative of three experimental replicates and error bars depict \pm 1 standard deviation. 


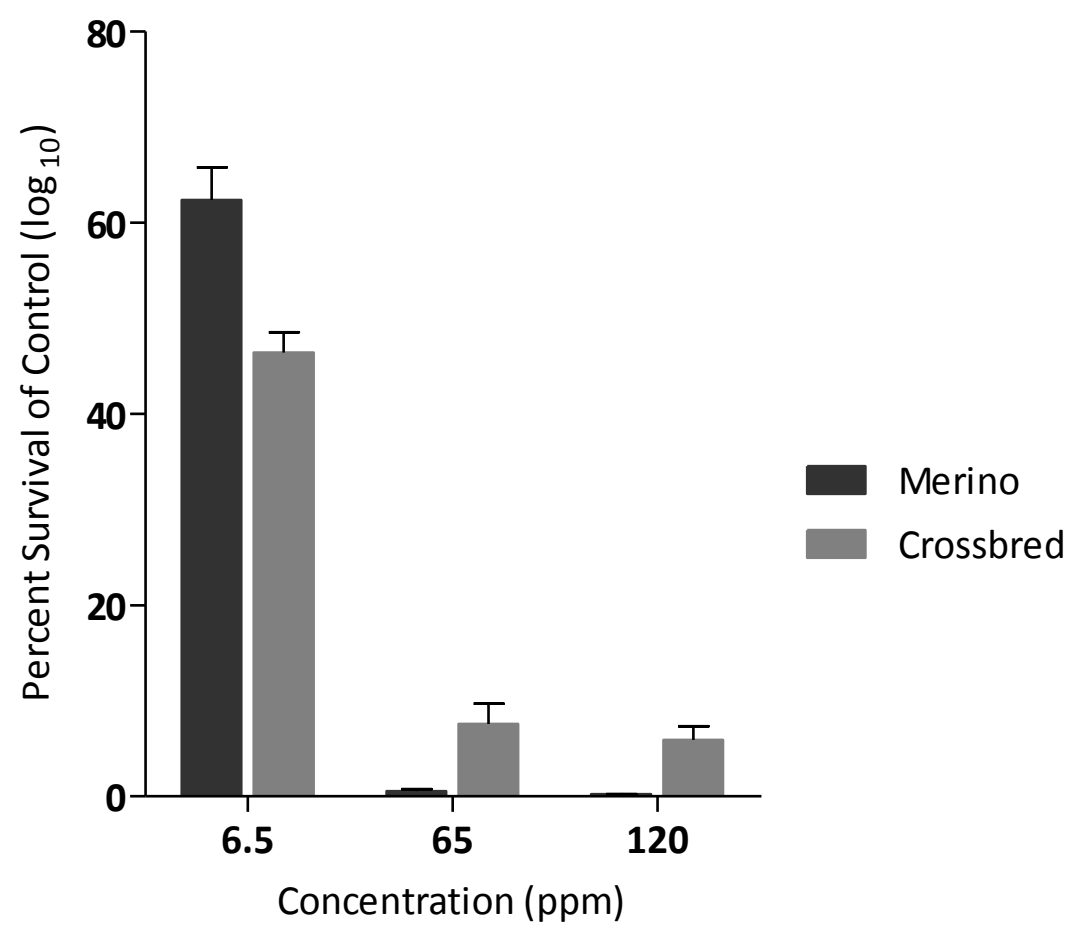

Figure A1.3. Merino- and crossbred-palladium composite biocidal activity of particles prepared at $80^{\circ} \mathrm{C}$

Nanopalladium merino or crossbred wool composites (6.5 ppm, $65 \mathrm{ppm}$, and $120 \mathrm{ppm}$ ) were prepared at $80^{\circ} \mathrm{C}$. The wool composites were treated for 7 days with PBS-washed $E$. coli. Bacteria were then quantified by CFU. The data presented is a representative of three experimental replicates and error bars depict \pm 1 standard deviation.

Bactericidal activity appeared to be positively related to palladium concentration. Particle sizes, relative to preparation temperature, were shown to range in diameter from $2-50 \mathrm{~nm}$ with the larger nanoparticles located mainly at the cuticle edges of the fibres. The larger the particle size the less bacterial killing was seen at any one palladium concentration. This would suggest that the greater the particle size the less palladium becomes available.

The merino fibre, smaller in diameter, possess a smoother surfaces structure than crossbred fibres. Therefore, by weight, more surface area is available on merino fibres for functional palladium groups to be absorbed and for particles to form. The observation by scanning electron microscopy suggested that merino imbedded particles were also more uniform in size and distribution among the fibres. This may indicate that some unknown characteristic of merino wool may also be contributing the antimicrobial qualities of the functional composite hybrids which will require additional investigation.

This project demonstrates that new hybrid materials of wool fibre and 
palladium nanoparticles can be successfully synthesized and control bacterial growth. Nanopalladium-wool fibres therefore provide the opportunity to develop new hybrid materials for application where the antibacterial properties are desired to prevent the proliferation and growth of bacteria that can cause diseases. 


\section{Appendix 2: Microarray Top Tables of Gene Expression}

Table A2.1. Top Table: Internal PAO1 vs. Control PAO1, up-regulated >3FC

\begin{tabular}{|c|c|c|c|c|c|}
\hline Gene ID & Name & $\begin{array}{c}\text { Fold } \\
\text { Change }\end{array}$ & P-value & Protein description and/or interspecies homology & Functional Class \\
\hline PA3126 & $i b p A$ & 12.6 & $8.86 \mathrm{E}-09$ & Heat-shock protein IbpA & Chaperones \& heat shock proteins \\
\hline PA3819 & & 8.05 & 4.97E-07 & Conserved hypothetical, $57 \%$ homologous to ycfJ (E. coli) & Hypothetical, unclassified, unknown; Membrane proteins \\
\hline PA4762 & $\operatorname{grp} E$ & 8.05 & $3.13 \mathrm{E}-08$ & Heat shock protein GrpE & $\begin{array}{l}\text { DNA replication, recombination, modification and repair; Chaperones \& } \\
\text { heat shock proteins }\end{array}$ \\
\hline PA3732 & & 7.79 & $3.38 \mathrm{E}-08$ & Conserved hypothetical & Hypothetical, unclassified, unknown \\
\hline ig_3874653_38738 & 835 & 7.21 & $9.89 \mathrm{E}-07$ & Intergenic region between PA3539 and PA3540 & Intergenic region between PA3539 and PA3540 \\
\hline PA0665 & & 6.67 & $2.36 \mathrm{E}-07$ & $\begin{array}{l}\text { Conserved hypothetical, } 86 \% \text { homologous to iron-sulfur cluster } \\
\text { insertion protein } \operatorname{ErpA}(\text { P. putida) }\end{array}$ & Hypothetical, unclassified, unknown \\
\hline PA5053 & $h s / V$ & 6.58 & $5.60 \mathrm{E}-08$ & Heat shock protein HsIV & Chaperones \& heat shock proteins \\
\hline PA3731 & & 6 & $5.60 \mathrm{E}-08$ & Conserved hypothetical, putative phage shock protein & Hypothetical, unclassified, unknown \\
\hline PA2604 & & 5.06 & 1.07E-06 & $\begin{array}{l}\text { Conserved hypothetical, putative TEGT family carrier/transport } \\
\text { protein, } 81 \% \text { homologous ( } P \text {. fluorescens) }\end{array}$ & Hypothetical, unclassified, unknown \\
\hline PA1592 & & 4.67 & $2.58 \mathrm{E}-07$ & Hypothetical protein, putative lipoprotein, cytochrome c class I & Hypothetical, unclassified, unknown \\
\hline PA0729 & & 4.47 & $3.12 \mathrm{E}-07$ & $\begin{array}{l}\text { Hypothetical protein, } 56 \% \text { homologous to plasmid stabilization } \\
\text { system protein (P. syringae) }\end{array}$ & Hypothetical, unclassified, unknown \\
\hline PA5470 & & 4.25 & $1.70 \mathrm{E}-08$ & Probable peptide chain release factor & Translation, post-translational modification, degradation \\
\hline PA3524 & gloA1 & 4.24 & $3.58 \mathrm{E}-07$ & Lactoylglutathione lyase & Central intermediary metabolism \\
\hline PA0763 & mucA & 4.17 & 1.07E-06 & Anti-sigma factor MucA & Transcriptional regulators \\
\hline PA3533 & & 4.03 & $5.37 \mathrm{E}-06$ & $\begin{array}{l}\text { Conserved hypothetical protein, } 90 \% \text { homologous glutaredoxin- } \\
\text { like protein }(P \text {. putida) }\end{array}$ & Hypothetical, unclassified, unknown \\
\hline PA3161 & $\operatorname{him} D$ & 4 & $3.59 \mathrm{E}-05$ & Integration host factor beta subunit & Cell wall / LPS / capsule \\
\hline PA3006 & & 3.98 & $9.06 \mathrm{E}-06$ & Probable transcriptional regulator & Transcriptional regulators \\
\hline PA4761 & dnak & 3.81 & $1.70 \mathrm{E}-08$ & DnaK protein & $\begin{array}{l}\text { Adaptation, protection; Chaperones \& heat shock proteins; DNA replication, } \\
\text { recombination, modification and repair }\end{array}$ \\
\hline
\end{tabular}




\begin{tabular}{|c|c|c|c|c|}
\hline PA3664 & & 3.81 & $1.36 \mathrm{E}-07$ & $\begin{array}{l}\text { Conserved hypothetical protein, } 81 \% \text { homologous to putative } \\
\text { oxidoreductase, FAD-binding protein ( } P \text {. putida) }\end{array}$ \\
\hline PA0762 & $\operatorname{alg} U$ & 3.74 & $5.36 \mathrm{E}-06$ & Sigma factor AlgU \\
\hline PA0376 & rpoH & 3.72 & $1.90 \mathrm{E}-06$ & Sigma factor RpoH \\
\hline PA3665 & & 3.53 & 3.33E-07 & Hypothetical protein, putative transporter, LysE family \\
\hline PA3351 & $f l g M$ & 3.5 & 2.77E-06 & $\begin{array}{l}\text { Hypothetical protein, negative regulator of flagellin synthesis } \\
\text { (anti-sigma28 factor) }\end{array}$ \\
\hline PA3815 & $i s c R$ & 3.49 & $1.83 \mathrm{E}-05$ & $\begin{array}{l}\text { Conserved hypothetical, iron-sulfur cluster assembly } \\
\text { transcription factor } I s c R\end{array}$ \\
\hline PA1596 & htpG & 3.29 & $5.62 \mathrm{E}-07$ & Heat shock protein HtpG \\
\hline PA2952 & etfB & 3.21 & $1.30 \mathrm{E}-06$ & Electron transfer flavoprotein beta-subunit \\
\hline PA4386 & groES & 3.2 & 4.71E-06 & GroES protein \\
\hline PA3183 & $z w f$ & 3.17 & 3.09E-07 & Glucose-6-phosphate 1-dehydrogenase \\
\hline PA5528 & & 3.07 & $2.13 \mathrm{E}-07$ & $\begin{array}{l}\text { Hypothetical protein, } 74 \% \text { homologous to import inner } \\
\text { membrane translocase, subunit Tim44 (P. syringae) }\end{array}$ \\
\hline PA5471 & & 3.02 & $1.36 \mathrm{E}-07$ & $\begin{array}{l}\text { Hypothetical protein, } 64 \% \text { homologous to RtcB (S. enterica) } \\
\text { distantly related to arsenate reductase }\end{array}$ \\
\hline PA4944 & $h f q$ & 3.02 & $7.08 \mathrm{E}-05$ & Conserved hypothetical protein \\
\hline
\end{tabular}

Hypothetical, unclassified, unknown

Transcriptional regulators

Transcriptional regulators

Hypothetical, unclassified, unknown; Membrane proteins

Hypothetical, unclassified, unknown

Hypothetical, unclassified, unknown

Chaperones \& heat shock proteins

Energy metabolism

Chaperones \& heat shock proteins

Carbon compound catabolism; Energy metabolism

Hypothetical, unclassified, unknown; Membrane proteins

Hypothetical, unclassified, unknown

Hypothetical, unclassified, unknown

\section{Table A2.2. Top Table: Internal PAO1 vs. Control PAO1, down-regulated >3 FC}

\begin{tabular}{|c|c|c|c|c|}
\hline Gene ID & Name & $\begin{array}{c}\text { Fold } \\
\text { Change }\end{array}$ & P-value & Protein description and/or interspecies homology \\
\hline PA0823 & & 6.89 & $1.70 \mathrm{E}-08$ & $\begin{array}{l}\text { Hypothetical protein, } 57 \% \text { homologous to VRR-NUC domain- } \\
\text { containing protein (P. syringae) }\end{array}$ \\
\hline PA0718 & & 6.5 & $2.62 \mathrm{E}-08$ & Hypothetical protein of bacteriophage Pf1 \\
\hline PA3868 & & 6.21 & $3.12 \mathrm{E}-07$ & $\begin{array}{l}\text { Hypothetical protein, } 82 \% \text { homologous to phage integrase } \\
\text { family site specific recombinase ( } P \text {. syringae) }\end{array}$ \\
\hline PA0499 & & 5.92 & $1.36 \mathrm{E}-07$ & Probable pili assembly chaperone \\
\hline PA0621 & & 5.9 & $1.70 \mathrm{E}-08$ & $\begin{array}{l}\text { Conserved hypothetical protein, putative tail fiber assembley } \\
\text { protein }\end{array}$ \\
\hline
\end{tabular}

Functional Class

Hypothetical, unclassified, unknown

Hypothetical, unclassified, unknown; Related to phage, transposon, or plasmid

Hypothetical, unclassified, unknown

Motility \& Attachment; Chaperones \& heat shock proteins

Related to phage, transposon, or plasmid 


\begin{tabular}{|c|c|c|c|c|}
\hline PA0260 & & 5.86 & $5.60 \mathrm{E}-08$ & Hypothetical protein \\
\hline PA1387 & & 5.39 & 5.79E-07 & Hypothetical protein \\
\hline PA0821 & & 5.1 & $2.36 \mathrm{E}-07$ & Hypothetical protein \\
\hline PA0978 & & 5.07 & 2.87E-07 & Conserved hypothetical protein, putative transposase \\
\hline PA0713 & & 5.06 & 1.29E-06 & Hypothetical protein \\
\hline PA2103 & & 5.04 & $5.59 \mathrm{E}-06$ & Probable molybdopterin biosynthesis protein MoeB \\
\hline PA0827 & & 4.88 & $1.36 \mathrm{E}-07$ & Hypothetical protein \\
\hline PA3520 & & 4.74 & 8.07E-07 & $\begin{array}{l}\text { Hypothetical protein, } 39 \% \text { homologous to heavy metal } \\
\text { transport/detoxification protein }(P . \text { putida) }\end{array}$ \\
\hline PA1388 & & 4.69 & $2.98 \mathrm{E}-06$ & Hypothetical protein \\
\hline PA0717 & & 4.67 & 1.77E-05 & Hypothetical protein of bacteriophage Pf1 \\
\hline PA0826 & & 4.59 & $1.27 \mathrm{E}-06$ & Hypothetical protein \\
\hline PA0574 & & 4.58 & $2.12 \mathrm{E}-06$ & $\begin{array}{l}\text { Hypothetical protein, } 87 \% \text { homologous to filamentation induced } \\
\text { by cAMP protein Fic (P. putida) }\end{array}$ \\
\hline PA0261 & & 4.42 & 1.37E-07 & Hypothetical protein \\
\hline PA0726 & & 4.42 & $1.34 \mathrm{E}-06$ & Hypothetical protein of bacteriophage Pf1 \\
\hline PA0979 & & 4.38 & 9.27E-06 & Conserved hypothetical protein, putative transposase \\
\hline PA1394 & & 4.36 & $3.38 \mathrm{E}-07$ & Hypothetical protein \\
\hline PA0992 & & 4.3 & $8.43 \mathrm{E}-06$ & Probable fimbrial subunit protein \\
\hline PA1150 & pys2 & 4.26 & $6.81 \mathrm{E}-06$ & Pyocin S2 \\
\hline PA1391 & & 4.26 & $3.88 \mathrm{E}-06$ & Probable glycosyl transferase \\
\hline PA1383 & & 4.15 & $6.53 \mathrm{E}-07$ & Hypothetical protein \\
\hline PA0727 & & 4.13 & $3.86 \mathrm{E}-07$ & Hypothetical protein from bacteriophage Pf1 \\
\hline PA1395 & & 4.12 & 3.69E-07 & Hypothetical protein \\
\hline PA1153 & & 4.12 & 4.89E-08 & Hypothetical protein \\
\hline PA4526 & pilB & 4.1 & 4.31E-07 & Type 4 fimbrial biogenesis protein PilB \\
\hline PA0819 & & 4.08 & $2.04 \mathrm{E}-07$ & Hypothetical protein \\
\hline PA4528 & pilD & 4.04 & $5.62 \mathrm{E}-07$ & Type 4 prepilin peptidase PilD \\
\hline
\end{tabular}

Membrane proteins; Hypothetical, unclassified, unknown

Hypothetical, unclassified, unknown

Hypothetical, unclassified, unknown

Related to phage, transposon, or plasmid

Hypothetical, unclassified, unknown

Biosynthesis of cofactors, prosthetic groups and carriers

Hypothetical, unclassified, unknown

Hypothetical, unclassified, unknown

Hypothetical, unclassified, unknown

Hypothetical, unclassified, unknown; Related to phage, transposon, or plasmid

Hypothetical, unclassified, unknown

Hypothetical, unclassified, unknown

Hypothetical, unclassified, unknown

Hypothetical, unclassified, unknown; Related to phage, transposon, or plasmid

Related to phage, transposon, or plasmid

Hypothetical, unclassified, unknown

Motility \& Attachment

Adaptation, protection; Secreted Factors (toxins, enzymes, alginate) Putative enzymes

Hypothetical, unclassified, unknown

Hypothetical, unclassified, unknown; Related to phage, transposon, or plasmid

Hypothetical, unclassified, unknown

Hypothetical, unclassified, unknown

Motility \& Attachment

Hypothetical, unclassified, unknown

Motility \& Attachment; Protein secretion/export apparatus; Secreted 


$\begin{array}{lcccl}\text { Pae_tRNA_Gly } & \text { tRNA } & 3.98 & 3.60 \mathrm{E}-05 & \text { tRNA_Glycine } \\ \text { PA1936 } & & 3.9 & 1.12 \mathrm{E}-06 & \text { Hypothetical protein } \\ \text { PA2104 } & 3.9 & 8.31 \mathrm{E}-06 & \text { Probable cysteine synthase } \\ \text { PA0257 } & & 3.86 & 3.38 \mathrm{E}-08 & \text { Hypothetical protein } \\ \text { PA1746 } & & 3.81 & 5.49 \mathrm{E}-06 & \text { Hypothetical protein } \\ \text { PA2570 } & 3.74 & 1.30 \mathrm{E}-06 & \text { PA-l galactophilic lectin } \\ \text { PA0730 } & & 3.73 & 8.93 \mathrm{E}-06 & \text { Probable transferase } \\ \text { PA1155 } & \text { nrdB } & 3.73 & 1.27 \mathrm{E}-06 & \text { Ribonucleoside reductase, small chain } \\ \text { PA0256 } & & 3.72 & 1.36 \mathrm{E}-07 & \text { Hypothetical protein } \\ \text { PA0263 } & \text { hcpC } & 3.72 & 9.33 \mathrm{E}-06 & \text { Secreted protein Hcp } \\ \text { PA2038 } & & 3.71 & 2.44 \mathrm{E}-06 & \text { Hypothetical protein } \\ \text { PA0982 } & & 3.7 & 4.66 \mathrm{E}-06 & \text { Hypothetical protein } \\ \text { PA0714 } & & 3.7 & 5.75 \mathrm{E}-07 & \text { Hypothetical protein } \\ \text { PA0824 } & & 3.69 & 3.38 \mathrm{E}-07 & \text { Hypothetical protein } \\ \text { PA1389 } & & 3.66 & 5.58 \mathrm{E}-07 & \text { Probable glycosyl transferase } \\ \text { PA1386 } & & 3.64 & 4.89 \mathrm{E}-05 & \text { Probable ATP-binding component of ABC transporter } \\ \text { PA3867 } & & 3.6 & 2.76 \mathrm{E}-05 & \text { Probable DNA invertase } \\ \text { PA0987 } & & 3.57 & 2.30 \mathrm{E}-06 & \text { Conserved hypothetical protein } \\ \text { PA0042 } & & 3.53 & 3.35 \mathrm{E}-06 & \text { Hypothetical protein } \\ \text { Pae_tRNA_Asnt } & t R N A & 3.43 & 1.45 \mathrm{E}-05 & \text { tRNA_Asparagine } \\ \text { PA2460 } & & 3.4 & 9.28 \mathrm{E}-05 & \text { Hypothetical protein } \\ \text { PA0620 } & & 3.4 & 3.75 \mathrm{E}-05 & \text { Probable bacteriophage protein } \\ \text { PA0264 } & & 3.38 & 6.28 \mathrm{E}-07 & \text { Hypothetical protein } \\ \text { PA2733 } & 3.36 & 1.12 \mathrm{E}-05 & \text { Conserved hypothetical protein } \\ \text { PA1149 } & 3.35 & 3.12 \mathrm{E}-07 & \text { Hypothetical protein } \\ \text { PA0728 } & & 3.34 & 5.95 \mathrm{E}-06 & \text { Probable bacteriophage integrase } \\ \text { PA0259 } & 3.33 & 1.86 \mathrm{E}-07 & \text { Hypothetical protein } \\ & & & \end{array}$

Factors (toxins, enzymes, alginate)

tRNA_Glycine

Hypothetical, unclassified, unknown

Amino acid biosynthesis and metabolism

Related to phage, transposon, or plasmid

Hypothetical, unclassified, unknown

Adaptation, protection; Motility \& Attachment

Putative enzymes

Nucleotide biosynthesis and metabolism

Hypothetical, unclassified, unknown

Secreted Factors (toxins, enzymes, alginate)

Hypothetical, unclassified, unknown

Hypothetical, unclassified, unknown

Hypothetical, unclassified, unknown

Hypothetical, unclassified, unknown

Putative enzymes

Transport of small molecules

DNA replication, recombination, modification and repair

Related to phage, transposon, or plasmid

Hypothetical, unclassified, unknown

tRNA_Asparagine

Hypothetical, unclassified, unknown

Related to phage, transposon, or plasmid

Hypothetical, unclassified, unknown

Hypothetical, unclassified, unknown

Hypothetical, unclassified, unknown

Putative enzymes; Related to phage, transposon, or plasmid

Hypothetical, unclassified, unknown 


\begin{tabular}{|c|c|c|c|c|}
\hline PA4239 & $\operatorname{rps} D$ & 3.32 & 1.27E-06 & $30 \mathrm{~S}$ ribosomal protein $\mathrm{S} 4$ \\
\hline PA3151 & hisF2 & 3.27 & $1.05 \mathrm{E}-05$ & Imidazoleglycerol-phosphate synthase, cyclase subunit \\
\hline PA0594 & surA & 3.25 & 4.25E-06 & Peptidyl-prolyl cis-trans isomerase SurA \\
\hline PA1317 & cyoA & 3.25 & $2.36 \mathrm{E}-07$ & Cytochrome o ubiquinol oxidase subunit II \\
\hline PA1393 & cysC & 3.24 & $9.29 \mathrm{E}-07$ & Adenosine 5'-phosphosulfate (APS) kinase \\
\hline ig_3526677_35274 & & 3.2 & $2.26 \mathrm{E}-06$ & Intergenic region between PA3159 and PA3160 \\
\hline PA4523 & & 3.17 & $1.68 \mathrm{E}-05$ & Hypothetical protein \\
\hline PA0820 & & 3.17 & $3.66 \mathrm{E}-05$ & Hypothetical protein \\
\hline PA0985 & & 3.14 & 5.59E-05 & Probable colicin-like toxin \\
\hline PA4238 & rpoA & 3.1 & $2.48 \mathrm{E}-05$ & DNA-directed RNA polymerase alpha chain \\
\hline PA4558 & & 3.09 & $8.43 E-06$ & Probable peptidyl-prolyl cis-trans isomerase, FkbP-type \\
\hline PA2670 & & 3.08 & $5.71 \mathrm{E}-06$ & Hypothetical protein \\
\hline PA0619 & & 3.07 & $1.36 \mathrm{E}-07$ & Probable bacteriophage protein \\
\hline PA1935 & & 3.07 & $6.82 \mathrm{E}-06$ & Hypothetical protein \\
\hline PA3488 & & 3.07 & 2.77E-07 & Hypothetical protein \\
\hline PA2105 & & 3.07 & 4.74E-05 & Probable acetyltransferase \\
\hline PA1390 & & 3.06 & $3.38 \mathrm{E}-06$ & Probable glycosyl transferase \\
\hline PA0818 & & 3.05 & $2.04 \mathrm{E}-07$ & Hypothetical protein \\
\hline PA2146 & & 3.04 & 1.05E-06 & Conserved hypothetical protein \\
\hline PA2577 & & 3.03 & $5.05 \mathrm{E}-07$ & Probable transcriptional regulator \\
\hline PA2580 & & 3.02 & $1.76 \mathrm{E}-05$ & Conserved hypothetical protein \\
\hline PA0817 & & 3.01 & $5.60 \mathrm{E}-08$ & Probable ring-cleaving dioxygenase \\
\hline PA0614 & & 3.01 & 2.87E-07 & Hypothetical protein \\
\hline PA0722 & & 3 & 4.73E-08 & Hypothetical protein of bacteriophage Pf1 \\
\hline
\end{tabular}

Translation, post-translational modification, degradation

Amino acid biosynthesis and metabolism

Adaptation, protection; Chaperones \& heat shock proteins; Translation,

post-translational modification, degradation

Energy metabolism

Central intermediary metabolism; Nucleotide biosynthesis and metabolism;

Amino acid biosynthesis and metabolism

Intergenic region between PA3159 and PA3160

Hypothetical, unclassified, unknown

Hypothetical, unclassified, unknown

Membrane proteins; Secreted Factors (toxins, enzymes, alginate)

Transcription, RNA processing and degradation

Chaperones \& heat shock proteins; Translation, post-translational modification, degradation

Hypothetical, unclassified, unknown

Related to phage, transposon, or plasmid

Hypothetical, unclassified, unknown

Hypothetical, unclassified, unknown

Putative enzymes

Putative enzymes

Hypothetical, unclassified, unknown

Hypothetical, unclassified, unknown

Hypothetical, unclassified, unknown; Membrane proteins

Putative enzymes

\section{Putative enzymes}

Hypothetical, unclassified, unknown

Hypothetical, unclassified, unknown; Related to phage, transposon, or plasmid 
Table A2.3. Top Table: External PAO1 vs. Control PAO1, up-regulated $>3$ FC

\begin{tabular}{|c|c|c|c|c|c|}
\hline Gene ID & Name & $\begin{array}{l}\text { Fold } \\
\text { Change }\end{array}$ & P-value & Protein description and/or interspecies homology & Functional Class \\
\hline PA1852 & & 5.36 & $1.85 \mathrm{E}-06$ & Hypothetical protein & Hypothetical, unclassified, unknown \\
\hline PA2412 & & 4.46 & $6.34 \mathrm{E}-06$ & $\begin{array}{l}\text { Conserved hypothetical protein, } 88 \% \text { homologous to } m b t H \\
\text { domain-containing protein }(P \text {. putida) }\end{array}$ & Hypothetical, unclassified, unknown \\
\hline Intergenic & & 4.34 & $1.22 \mathrm{E}-05$ & Intergenic region between PA3539 and PA3540 & Intergenic region between PA3539 and PA3540 \\
\hline PA3151 & hisF2 & 4.29 & 8.57E-06 & Imidazoleglycerol-phosphate synthase, cyclase subunit & Amino acid biosynthesis and metabolism \\
\hline PA3533 & & 4.22 & $1.16 \mathrm{E}-05$ & Conserved hypothetical protein, glutaredoxin-related protein & Hypothetical, unclassified, unknown \\
\hline Pae_tRNA_Tyr & $t R N A$ & 4.21 & 7.63E-06 & tRNA_Tyrosine & tRNA_Tyrosine \\
\hline Pae_tRNA_Asn & tRNA & 4.17 & $1.38 \mathrm{E}-05$ & tRNA_Asparagine & tRNA_Asparagine \\
\hline Pae_tRNA_Lys & tRNA & 3.82 & 7.10E-06 & tRNA_Lysine & tRNA_Lysine \\
\hline PA3266 & $\operatorname{cap} B$ & 3.7 & $2.04 \mathrm{E}-05$ & Cold acclimation protein B & Adaptation, protection; Transcriptional regulators \\
\hline PA0665 & & 3.62 & 7.10E-06 & $\begin{array}{l}\text { Conserved hypothetical protein, } 86 \% \text { homologous to iron-sulfur } \\
\text { cluster insertion protein ErpA (P. putida) }\end{array}$ & Hypothetical, unclassified, unknown \\
\hline PA0579 & rpsU & 3.49 & 7.57E-06 & $30 S$ ribosomal protein $\mathrm{S} 21$ & Hypothetical, unclassified, unknown \\
\hline PA3621 & $f d x A$ & 3.39 & 7.73E-06 & Ferredoxin I & Energy metabolism \\
\hline Pae_tRNA_Gly & $t R N A$ & 3.38 & $1.26 \mathrm{E}-04$ & tRNA_Glycine & tRNA_Glycine \\
\hline PA2967 & $f a b G$ & 3.26 & $1.26 \mathrm{E}-06$ & 3-oxoacyl-[acyl-carrier-protein] reductase & Fatty acid and phospholipid metabolism \\
\hline PA4753 & & 3.19 & 1.47E-05 & Conserved hypothetical protein, putative RNA-binding protein & Hypothetical, unclassified, unknown \\
\hline PA4466 & & 3.13 & $3.32 \mathrm{E}-06$ & Probable phosphoryl carrier protein & Transport of small molecules \\
\hline PA2667 & mvaU & 3.07 & 7.10E-06 & $\begin{array}{l}\text { Conserved hypothetical protein, putative transcriptional } \\
\text { regulator }\end{array}$ & Transcriptional regulators \\
\hline PA5049 & rpmE & 3 & 3.19E-04 & $50 \mathrm{~S}$ ribosomal protein $\mathrm{L} 31$ & Translation, post-translational modification, degradation \\
\hline PA2970 & & 3 & $3.34 \mathrm{E}-04$ & $50 S$ ribosomal protein $L 32$ & Translation, post-translational modification, degradation \\
\hline
\end{tabular}


Table A2.4. Top Table: External PAO1 vs. Control PAO1, down-regulated >3 FC

\begin{tabular}{|c|c|c|c|c|c|}
\hline Gene ID & Name & $\begin{array}{c}\text { Fold } \\
\text { Change }\end{array}$ & P-value & Protein description and/or interspecies homology & Functional Class \\
\hline PA0718 & & 4.16 & $9.57 \mathrm{E}-07$ & Hypothetical protein of bacteriophage Pf1 & $\begin{array}{l}\text { Hypothetical, unclassified, unknown; Related to phage, transposon, or } \\
\text { plasmid }\end{array}$ \\
\hline PA0717 & & 4.01 & $5.66 \mathrm{E}-05$ & Hypothetical protein of bacteriophage Pf1 & $\begin{array}{l}\text { Hypothetical, unclassified, unknown; Related to phage, transposon, or } \\
\text { plasmid }\end{array}$ \\
\hline PA1746 & & 3.98 & $1.18 \mathrm{E}-05$ & Hypothetical protein & Hypothetical, unclassified, unknown \\
\hline PA2146 & & 3.96 & $1.63 E-06$ & $\begin{array}{l}\text { Conserved hypothetical protein, } 83 \% \text { homologous to yciG ( } K \text {. } \\
\text { pneumoniae) positively regulated by rpos }\end{array}$ & Hypothetical, unclassified, unknown \\
\hline PA1387 & & 3.25 & $1.18 \mathrm{E}-05$ & Hypothetical protein & Hypothetical, unclassified, unknown \\
\hline PA3049 & $r m f$ & 3.24 & $3.58 \mathrm{E}-05$ & Ribosome modulation factor & Translation, post-translational modification, degradation \\
\hline PA0713 & & 3.24 & $1.71 \mathrm{E}-05$ & Hypothetical protein & Hypothetical, unclassified, unknown \\
\hline PA0714 & & 3.21 & $5.42 \mathrm{E}-06$ & Hypothetical protein phrD & Hypothetical, unclassified, unknown \\
\hline PA0728 & & 3.15 & $1.72 \mathrm{E}-05$ & Probable bacteriophage integrase & Putative enzymes; Related to phage, transposon, or plasmid \\
\hline PA0726 & & 3.09 & $1.52 \mathrm{E}-05$ & Hypothetical protein of bacteriophage Pf1 & $\begin{array}{l}\text { Hypothetical, unclassified, unknown; Related to phage, transposon, or } \\
\text { plasmid }\end{array}$ \\
\hline Gene ID & Name & $\begin{array}{c}\text { Fold } \\
\text { Change }\end{array}$ & P-value & Protein description and/or interspecies homology & Functional Class \\
\hline PA3126 & $i b p a$ & 32.54 & $1.45 \mathrm{E}-08$ & Heat-shock protein IbpA & Chaperones \& heat shock proteins \\
\hline PA3732 & & 9.71 & $1.48 \mathrm{E}-08$ & Conserved hypothetical protein & Hypothetical, unclassified, unknown \\
\hline PA4762 & grpE & 9.66 & $1.45 \mathrm{E}-08$ & Heat shock protein GrpE & $\begin{array}{l}\text { DNA replication, recombination, modification and repair; Chaperones \& } \\
\text { heat shock proteins }\end{array}$ \\
\hline PA3731 & & 8.06 & $4.51 \mathrm{E}-08$ & Conserved hypothetical protein & Hypothetical, unclassified, unknown \\
\hline PA3819 & & 6.05 & $1.05 \mathrm{E}-06$ & Conserved hypothetical protein & Hypothetical, unclassified, unknown; Membrane proteins \\
\hline PA1029 & & 5.87 & 7.93E-08 & Hypothetical protein & Hypothetical, unclassified, unknown \\
\hline PA5053 & $h s / V$ & 5.58 & $3.61 \mathrm{E}-08$ & Heat shock protein HsIV & Chaperones \& heat shock proteins \\
\hline PA5470 & & 5.25 & $3.95 \mathrm{E}-08$ & Probable peptide chain release factor & Translation, post-translational modification, degradation \\
\hline
\end{tabular}




$\begin{array}{lllll}\text { PA4761 } & \text { dnak } & 5.18 & 1.78 \mathrm{E}-08 & \text { DnaK protein } \\ \text { PA0763 } & \text { mucA } & 4.99 & 1.48 \mathrm{E}-07 & \text { Anti-sigma factor MucA } \\ \text { PA2604 } & & 4.91 & 1.41 \mathrm{E}-07 & \text { Conserved hypothetical protein } \\ \text { PA1596 } & \text { htpG } & 4.61 & 3.59 \mathrm{E}-07 & \text { Heat shock protein HtpG } \\ \text { PA5471 } & & 4.06 & 2.54 \mathrm{E}-07 & \text { Hypothetical protein } \\ \text { PA0729 } & & 4.01 & 2.27 \mathrm{E}-08 & \text { Hypothetical protein } \\ \text { PA3007 } & \text { lexA } & 3.77 & 3.29 \mathrm{E}-07 & \text { Repressor protein LexA } \\ \text { PA4542 } & \text { clpB } & 3.71 & 7.93 \mathrm{E}-08 & \text { ClpB protein } \\ \text { PA2952 } & \text { etfB } & 3.46 & 1.65 \mathrm{E}-07 & \text { Electron transfer flavoprotein beta-subunit } \\ \text { PA4739 } & & 3.41 & 1.92 \mathrm{E}-07 & \text { Conserved hypothetical protein } \\ \text { PA0762 } & \text { algU } & 3.38 & 2.45 \mathrm{E}-06 & \text { Sigma factor AlgU } \\ \text { PA5528 } & & 3.33 & 1.97 \mathrm{E}-06 & \text { Hypothetical protein } \\ \text { PA4386 } & \text { groES } & 3.27 & 8.28 \mathrm{E}-08 & \text { GroES protein } \\ \text { PA0610 } & \text { prtN } & 3.09 & 1.31 \mathrm{E}-06 & \text { Transcriptional regulator PrtN } \\ \text { PA3006 } & & 3.01 & 1.05 \mathrm{E}-06 & \text { Probable transcriptional regulator }\end{array}$

Adaptation, protection; Chaperones \& heat shock proteins; DNA replication, recombination, modification and repair

Transcriptional regulators

Hypothetical, unclassified, unknown

Chaperones \& heat shock proteins

Hypothetical, unclassified, unknown

Hypothetical, unclassified, unknown

Adaptation, protection; Translation, post-translational modification,

degradation

Translation, post-translational modification, degradation

Energy metabolism

Hypothetical, unclassified, unknown

Transcriptional regulators

Hypothetical, unclassified, unknown; Membrane proteins

Chaperones \& heat shock proteins

Transcriptional regulators

Transcriptional regulators

Table A2.6. Top Table: Internal PAO1 vs. External PA01, down-regulated >3 FC

\begin{tabular}{|c|c|c|c|c|c|}
\hline Gene ID & Name & $\begin{array}{c}\text { Fold } \\
\text { Change }\end{array}$ & P-value & Protein description and/or interspecies homology & Functional Class \\
\hline PA3151 & hisF2 & 20.98 & $1.45 \mathrm{E}-08$ & Imidazoleglycerol-phosphate synthase, cyclase subunit & Amino acid biosynthesis and metabolism \\
\hline Pae_tRNA_Asn & $t R N A$ & 19.7 & 1.87E-08 & tRNA_Asparagine & tRNA_Asparagine \\
\hline Pae_tRNA_Gly & tRNA & 16.68 & $2.16 \mathrm{E}-08$ & tRNA_Glycine & tRNA_Glycine \\
\hline Pae_tRNA_Tyr & $t R N A$ & 10.86 & $3.78 \mathrm{E}-07$ & tRNA_Tyrosine & tRNA_Tyrosine \\
\hline PA3150 & $w b p G$ & 9.7 & $1.41 \mathrm{E}-07$ & LPS biosynthesis protein WbpG & Cell wall / LPS / capsule \\
\hline PA3149 & $w b p H$ & 8.8 & $1.65 \mathrm{E}-07$ & Probable glycosyltransferase $\mathrm{WbpH}$ & Cell wall / LPS / capsule; Putative enzymes \\
\hline PA3152 & hisH2 & 8.7 & $1.55 \mathrm{E}-08$ & Glutamine amidotransferase & Amino acid biosynthesis and metabolism \\
\hline PA2460 & & 8.29 & $1.48 \mathrm{E}-08$ & Hypothetical protein & Hypothetical, unclassified, unknown \\
\hline
\end{tabular}




\begin{tabular}{|c|c|c|c|c|}
\hline PA4239 & $r p s D$ & 7.39 & $1.60 \mathrm{E}-07$ & $30 S$ ribosomal protein $\mathrm{S} 4$ \\
\hline PA0252 & & 7.35 & $8.46 \mathrm{E}-08$ & Hypothetical protein \\
\hline PA2970 & $r p m F$ & 7.05 & $2.76 \mathrm{E}-06$ & $50 S$ ribosomal protein $\mathrm{L} 32$ \\
\hline PA4525 & pilA & 6.33 & $1.89 \mathrm{E}-07$ & Type 4 fimbrial precursor PilA \\
\hline PA3148 & wbpl & 6.2 & $5.86 \mathrm{E}-07$ & Probable UDP-N-acetylglucosamine 2-epimerase Wbpl \\
\hline PA2386 & $p v d A$ & 6.16 & $3.29 \mathrm{E}-07$ & L-ornithine N5-oxygenase \\
\hline PA0985 & & 6.05 & 4.07E-05 & Probable colicin-like toxin \\
\hline PA4238 & rpoA & 5.99 & $2.64 \mathrm{E}-07$ & DNA-directed RNA polymerase alpha chain \\
\hline PA4237 & rplQ & 5.87 & $8.28 \mathrm{E}-08$ & $50 S$ ribosomal protein $\mathrm{L} 17$ \\
\hline PA5568 & & 5.85 & $1.48 \mathrm{E}-08$ & Conserved hypothetical protein \\
\hline PA4247 & $r p / R$ & 5.75 & $5.84 \mathrm{E}-06$ & $50 S$ ribosomal protein L18 \\
\hline PA4242 & rpmJ & 5.35 & $1.29 \mathrm{E}-05$ & $50 S$ ribosomal protein L36 \\
\hline PA0579 & $r p s U$ & 5.35 & $1.05 \mathrm{E}-06$ & $30 S$ ribosomal protein S21 \\
\hline PA5555 & $\operatorname{atpG}$ & 5.34 & $1.55 \mathrm{E}-08$ & ATP synthase gamma chain \\
\hline PA2730 & & 5.31 & $6.20 \mathrm{E}-07$ & Hypothetical protein \\
\hline PA2761 & & 5.31 & $1.01 \mathrm{E}-06$ & Hypothetical protein \\
\hline PA4245 & $r p m D$ & 5.29 & $3.78 \mathrm{E}-07$ & $50 S$ ribosomal protein L30 \\
\hline PA4240 & rpsK & 5.23 & $1.71 \mathrm{E}-07$ & $30 S$ ribosomal protein S11 \\
\hline PA1431 & $r s a L$ & 5.17 & $1.65 \mathrm{E}-07$ & Regulatory protein RsaL \\
\hline PA1713 & exs $A$ & 5.14 & $1.26 \mathrm{E}-07$ & Transcriptional regulator ExsA \\
\hline PA2398 & $f p v A$ & 5.13 & $1.43 \mathrm{E}-05$ & Ferripyoverdine receptor \\
\hline PA3266 & $c a p B$ & 5.02 & $1.86 \mathrm{E}-06$ & Cold acclimation protein B \\
\hline PA4407 & ftsZ & 4.94 & $1.65 \mathrm{E}-07$ & Cell division protein FtsZ \\
\hline PA1155 & $n r d B$ & 4.88 & 7.93E-06 & Ribonucleoside reductase, small chain \\
\hline PA2412 & & 4.82 & $6.37 \mathrm{E}-06$ & Conserved hypothetical protein \\
\hline PA1159 & & 4.82 & $2.03 \mathrm{E}-07$ & Probable cold-shock protein \\
\hline PA5562 & spoOJ & 4.79 & $1.55 \mathrm{E}-08$ & Chromosome partitioning protein SpoOJ \\
\hline
\end{tabular}

Translation, post-translational modification, degradation Hypothetical, unclassified, unknown

Translation, post-translational modification, degradation Motility \& Attachment

Cell wall / LPS / capsule; Putative enzymes

Adaptation, protection

Membrane proteins; Secreted Factors (toxins, enzymes, alginate)

Transcription, RNA processing and degradation

Translation, post-translational modification, degradation Hypothetical, unclassified, unknown; Membrane proteins Translation, post-translational modification, degradation Translation, post-translational modification, degradation Hypothetical, unclassified, unknown

Energy metabolism

Hypothetical, unclassified, unknown

Hypothetical, unclassified, unknown; Membrane proteins Translation, post-translational modification, degradation Translation, post-translational modification, degradation Adaptation, protection; Transcriptional regulators; Secreted Factors (toxins, enzymes, alginate)

Protein secretion/export apparatus; Transcriptional regulators

Transport of small molecules

Adaptation, protection; Transcriptional regulators

Cell division

Nucleotide biosynthesis and metabolism

Hypothetical, unclassified, unknown

Adaptation, protection; Transcriptional regulators

Cell division 


\begin{tabular}{|c|c|c|c|c|}
\hline PA1150 & pys2 & 4.76 & $3.59 \mathrm{E}-07$ & Pyocin S2 \\
\hline PA4246 & rpsE & 4.74 & $2.44 \mathrm{E}-06$ & $30 \mathrm{~S}$ ribosomal protein $\mathrm{S} 5$ \\
\hline PA5554 & $a t p D$ & 4.74 & 1.69E-07 & ATP synthase beta chain \\
\hline PA4670 & prs & 4.63 & $5.40 \mathrm{E}-07$ & Ribose-phosphate pyrophosphokinase \\
\hline PA2967 & $f a b G$ & 4.62 & $1.69 \mathrm{E}-07$ & 3-oxoacyl-[acyl-carrier-protein] reductase \\
\hline PA4558 & & 4.61 & $5.12 \mathrm{E}-06$ & Probable peptidyl-prolyl cis-trans isomerase, FkbP-type \\
\hline PA1852 & & 4.6 & $5.30 \mathrm{E}-07$ & Hypothetical protein \\
\hline PA0668 & tyrZ & 4.57 & $8.25 \mathrm{E}-06$ & Tyrosyl-tRNA synthetase 2 \\
\hline PA5556 & $\operatorname{atpA}$ & 4.57 & $3.58 \mathrm{E}-06$ & ATP synthase alpha chain \\
\hline PA1584 & $s d h B$ & 4.53 & $5.98 \mathrm{E}-06$ & Succinate dehydrogenase (B subunit) \\
\hline PA1588 & succ & 4.51 & $1.18 \mathrm{E}-06$ & Succinyl-CoA synthetase beta chain \\
\hline PA4272 & $r p / J$ & 4.48 & $9.02 \mathrm{E}-08$ & $50 \mathrm{~S}$ ribosomal protein $\mathrm{L} 10$ \\
\hline PA2731 & & 4.42 & $1.43 \mathrm{E}-05$ & Hypothetical protein \\
\hline PA4254 & $r p s Q$ & 4.4 & 2.49E-06 & $30 \mathrm{~S}$ ribosomal protein S17 \\
\hline PA4526 & pilB & 4.4 & $3.78 \mathrm{E}-07$ & Type 4 fimbrial biogenesis protein PilB \\
\hline PA3645 & $f a b Z$ & 4.37 & $1.33 \mathrm{E}-07$ & (3R)-hydroxymyristoyl-[acyl carrier protein] dehydratase \\
\hline PA4429 & & 4.34 & $3.62 \mathrm{E}-07$ & Probable cytochrome c1 precursor \\
\hline PA4671 & & 4.34 & $3.86 \mathrm{E}-06$ & Probable ribosomal protein $\mathrm{L} 25$ \\
\hline PA4269 & rpoc & 4.27 & $9.43 \mathrm{E}-07$ & DNA-directed RNA polymerase beta* chain \\
\hline PA1776 & & 4.24 & $1.06 \mathrm{E}-07$ & Probable sigma-70 factor, ECF subfamily \\
\hline PA4528 & pilD & 4.24 & $1.15 \mathrm{E}-06$ & Type 4 prepilin peptidase PilD \\
\hline PA3809 & $f d \times 2$ & 4.23 & 3.39E-07 & Ferredoxin [2Fe-2S] \\
\hline PA2396 & & 4.21 & $1.89 \mathrm{E}-07$ & Hypothetical protein \\
\hline Pae_tRNA_Lys & tRNA & 4.19 & $5.69 \mathrm{E}-06$ & tRNA_Lysine \\
\hline PA0594 & surA & 4.18 & $9.43 \mathrm{E}-07$ & Peptidyl-prolyl cis-trans isomerase SurA \\
\hline PA4248 & $r p / F$ & 4.17 & 8.41E-07 & 50 S ribosomal protein $\mathrm{L} 6$ \\
\hline
\end{tabular}

Adaptation, protection; Secreted Factors (toxins, enzymes, alginate)

Translation, post-translational modification, degradation

Energy metabolism

Carbon compound catabolism; Nucleotide biosynthesis and metabolism Fatty acid and phospholipid metabolism

Chaperones \& heat shock proteins; Translation, post-translational

modification, degradation

Hypothetical, unclassified, unknown

Amino acid biosynthesis and metabolism; Translation, post-translational modification, degradation

Energy metabolism

Energy metabolism

Energy metabolism

Translation, post-translational modification, degradation

Hypothetical, unclassified, unknown

Translation, post-translational modification, degradation

Motility \& Attachment

Cell wall / LPS / capsule; Fatty acid and phospholipid metabolism

Energy metabolism

Adaptation, protection; Translation, post-translational modification,

degradation

Transcription, RNA processing and degradation

Transcriptional regulators

Motility \& Attachment; Protein secretion/export apparatus; Secreted

Factors (toxins, enzymes, alginate)

Energy metabolism

Hypothetical, unclassified, unknown

tRNA_Lysine

Adaptation, protection; Chaperones \& heat shock proteins; Translation, post-translational modification, degradation

Translation, post-translational modification, degradation 


\begin{tabular}{|c|c|c|c|c|c|}
\hline PA4241 & $r p s M$ & 4.17 & 5.53E-06 & $30 \mathrm{~S}$ ribosomal protein S13 & Translation, post-translational modification, degradation \\
\hline PA1553 & & 4.12 & $1.65 \mathrm{E}-07$ & Probable cytochrome c oxidase subunit & Energy metabolism \\
\hline PA4466 & & 4.11 & $2.54 \mathrm{E}-07$ & Probable phosphoryl carrier protein & Transport of small molecules \\
\hline PA2733 & & 4.07 & $1.29 \mathrm{E}-05$ & Conserved hypothetical protein & Hypothetical, unclassified, unknown \\
\hline PA3158 & $w b p B$ & 4.06 & $1.66 \mathrm{E}-07$ & Probable oxidoreductase WpbB & Cell wall / LPS / capsule; Putative enzymes \\
\hline PA0456 & & 3.98 & 2.77E-07 & Probable cold-shock protein & Adaptation, protection; Transcriptional regulators \\
\hline PA1710 & excC & 3.97 & $5.81 \mathrm{E}-05$ & Exoenzyme $\mathrm{S}$ synthesis protein $\mathrm{C}$ precursor & $\begin{array}{l}\text { Protein secretion/export apparatus; Translation, post-translational } \\
\text { modification, degradation }\end{array}$ \\
\hline Intergenic & & 3.96 & $1.01 \mathrm{E}-06$ & Intergenic region between PA3159 and PA3160 & Intergenic region between PA3159 and PA3160 \\
\hline PA4244 & rplO & 3.92 & $3.62 \mathrm{E}-07$ & $50 S$ ribosomal protein L15 & Translation, post-translational modification, degradation \\
\hline PA4243 & $\sec Y$ & 3.89 & $6.09 \mathrm{E}-07$ & Secretion protein SecY & Membrane proteins; Protein secretion/export apparatus \\
\hline PA1151 & $i m m 2$ & 3.81 & $5.93 E-06$ & Pyocin S2 immunity protein & Adaptation, protection \\
\hline PA4694 & $i l v C$ & 3.8 & $2.96 \mathrm{E}-05$ & Ketol-acid reductoisomerase & $\begin{array}{l}\text { Amino acid biosynthesis and metabolism; Biosynthesis of cofactors, } \\
\text { prosthetic groups and carriers }\end{array}$ \\
\hline PA2667 & & 3.73 & $9.43 \mathrm{E}-07$ & Conserved hypothetical protein & Transcriptional regulators \\
\hline PA3147 & $w b p J$ & 3.68 & $2.83 \mathrm{E}-06$ & Probable glycosyl transferase WbpJ & Putative enzymes; Cell wall / LPS / capsule \\
\hline PA3163 & $\mathrm{cmk}$ & 3.66 & $5.73 \mathrm{E}-06$ & Cytidylate kinase & Nucleotide biosynthesis and metabolism \\
\hline PA4271 & $r p / L$ & 3.63 & $1.69 \mathrm{E}-07$ & $50 S$ ribosomal protein L7 / L12 & Translation, post-translational modification, degradation \\
\hline Intergenic & & 3.62 & 4.12E-06 & Intergenic region between PA4581 and PA4582 & Intergenic region between PA4581 and PA4582 \\
\hline Intergenic & & 3.58 & $1.02 \mathrm{E}-05$ & Intergenic region between PA2583 and PA2584 & Intergenic region between PA2583 and PA2584 \\
\hline PA1589 & $\operatorname{sucD}$ & 3.57 & $2.24 \mathrm{E}-06$ & Succinyl-CoA synthetase alpha chain & Energy metabolism \\
\hline PA4550 & fimU & 3.54 & $3.60 \mathrm{E}-05$ & Type 4 fimbrial biogenesis protein FimU & Motility \& Attachment \\
\hline PA2397 & $p v d E$ & 3.53 & 5.79E-05 & Pyoverdine biosynthesis protein $\mathrm{PvdE}$ & Adaptation, protection; Membrane proteins; Transport of small molecules \\
\hline PA4266 & fusA1 & 3.49 & $6.07 \mathrm{E}-06$ & Elongation factor $\mathrm{G}$ & Translation, post-translational modification, degradation \\
\hline PA1554 & & 3.45 & $3.16 \mathrm{E}-06$ & Probable cytochrome oxidase subunit (cbb3-type) & Energy metabolism \\
\hline PA3190 & & 3.42 & $6.47 \mathrm{E}-07$ & Probable binding protein component of $A B C$ sugar transporter & Transport of small molecules \\
\hline PA0260 & & 3.41 & $2.00 \mathrm{E}-07$ & Hypothetical protein & Membrane proteins; Hypothetical, unclassified, unknown \\
\hline PA2394 & & 3.37 & $1.52 \mathrm{E}-06$ & Probable aminotransferase & Putative enzymes \\
\hline PA4275 & nus $G$ & 3.36 & $3.32 \mathrm{E}-07$ & Transcription antitermination protein NusG & Transcription, RNA processing and degradation \\
\hline
\end{tabular}




\begin{tabular}{|c|c|c|c|c|}
\hline Pae_tRNA_Leu & $t R N A$ & 3.35 & $3.59 \mathrm{E}-07$ & tRNA_Leucine \\
\hline PA3155 & $w b p E$ & 3.35 & 8.93E-07 & Probable aminotransferase WbpE \\
\hline PA2619 & $\operatorname{infA}$ & 3.34 & $5.40 \mathrm{E}-07$ & Initiation factor \\
\hline PA0982 & & 3.33 & 4.21E-06 & Hypothetical protein \\
\hline PA3644 & Ipxa & 3.33 & $5.45 \mathrm{E}-06$ & UDP-N-acetylglucosamine acyltransferase \\
\hline PA3646 & IpxD & 3.31 & 3.54E-06 & UDP-3-O-[3-hydroxylauroyl] glucosamine $\mathrm{N}$-acyltransferase \\
\hline PA5553 & $\operatorname{atpC}$ & 3.31 & $9.36 \mathrm{E}-06$ & ATP synthase epsilon chain \\
\hline PA4672 & & 3.3 & $5.34 \mathrm{E}-06$ & Peptidyl-tRNA hydrolase \\
\hline PA2453 & & 3.27 & $1.66 \mathrm{E}-06$ & Hypothetical protein \\
\hline PA3046 & & 3.26 & 7.91E-07 & Conserved hypothetical protein \\
\hline PA2950 & & 3.25 & $4.66 \mathrm{E}-07$ & Hypothetical protein \\
\hline PA0499 & & 3.24 & $6.17 \mathrm{E}-05$ & Probable pili assembly chaperone \\
\hline PA4563 & rpst & 3.22 & $8.95 \mathrm{E}-07$ & 30 S ribosomal protein S20 \\
\hline PA0823 & & 3.19 & $5.35 \mathrm{E}-07$ & Hypothetical protein \\
\hline PA3162 & rpsA & 3.17 & 2.77E-07 & $30 S$ ribosomal protein S1 \\
\hline Intergenic & & 3.16 & $3.71 \mathrm{E}-07$ & Intergenic region between PA0668 and PA0669 \\
\hline PA4276 & secE & 3.16 & $1.93 \mathrm{E}-06$ & Secretion protein SecE \\
\hline PA1533 & & 3.16 & $1.12 \mathrm{E}-06$ & Conserved hypothetical protein \\
\hline PA0821 & & 3.16 & $1.55 \mathrm{E}-06$ & Hypothetical protein \\
\hline PA0992 & & 3.15 & $1.13 \mathrm{E}-05$ & Probable fimbrial subunit protein \\
\hline PA0970 & tolR & 3.15 & $2.26 \mathrm{E}-05$ & TolR protein \\
\hline PA4441 & & 3.14 & $2.09 \mathrm{E}-06$ & Hypothetical protein \\
\hline PA0837 & slyD & 3.13 & $3.54 \mathrm{E}-06$ & Peptidyl-prolyl cis-trans isomerase SlyD \\
\hline Pae_tRNA_Trp & $t R N A$ & 3.11 & 7.75E-06 & tRNA_Tryptophan \\
\hline PA0820 & & 3.04 & $1.10 \mathrm{E}-06$ & Hypothetical protein \\
\hline PA3531 & $b f r B$ & 3.02 & $8.96 \mathrm{E}-06$ & Bacterioferritin \\
\hline PA4273 & $r p / A$ & 3 & $5.78 \mathrm{E}-06$ & $50 S$ ribosomal protein $\mathrm{L} 1$ \\
\hline
\end{tabular}

tRNA_Leucine

Cell wall / LPS / capsule; Putative enzymes

Translation, post-translational modification, degradation Hypothetical, unclassified, unknown

Cell wall / LPS / capsule

Cell wall / LPS / capsule

Energy metabolism

Translation, post-translational modification, degradation

Hypothetical, unclassified, unknown

Hypothetical, unclassified, unknown

Hypothetical, unclassified, unknown

Motility \& Attachment; Chaperones \& heat shock proteins

Translation, post-translational modification, degradation; Central intermediary metabolism

Hypothetical, unclassified, unknown

Translation, post-translational modification, degradation

Intergenic region between PA0668 and PA0669

Protein secretion/export apparatus

Hypothetical, unclassified, unknown

Hypothetical, unclassified, unknown

Motility \& Attachment

Transport of small molecules

Hypothetical, unclassified, unknown

Chaperones \& heat shock proteins; Translation, post-translational modification, degradation

tRNA_Tryptophan

Hypothetical, unclassified, unknown

Transport of small molecules; Adaptation, protection

Translation, post-translational modification, degradation 


\section{Appendix 3: Microarray Data Sets by Functional Classification}

Genes with >2 FC expression compared to Control PAO1 are listed by functional annotation as a percentage of annotation occurrence within the data set.

"Hypothetical, unclassified, unknown" genes were excluded from this analysis.

Figure A3.1. Percent Internal PAO1 up-regulated vs. down-regulated

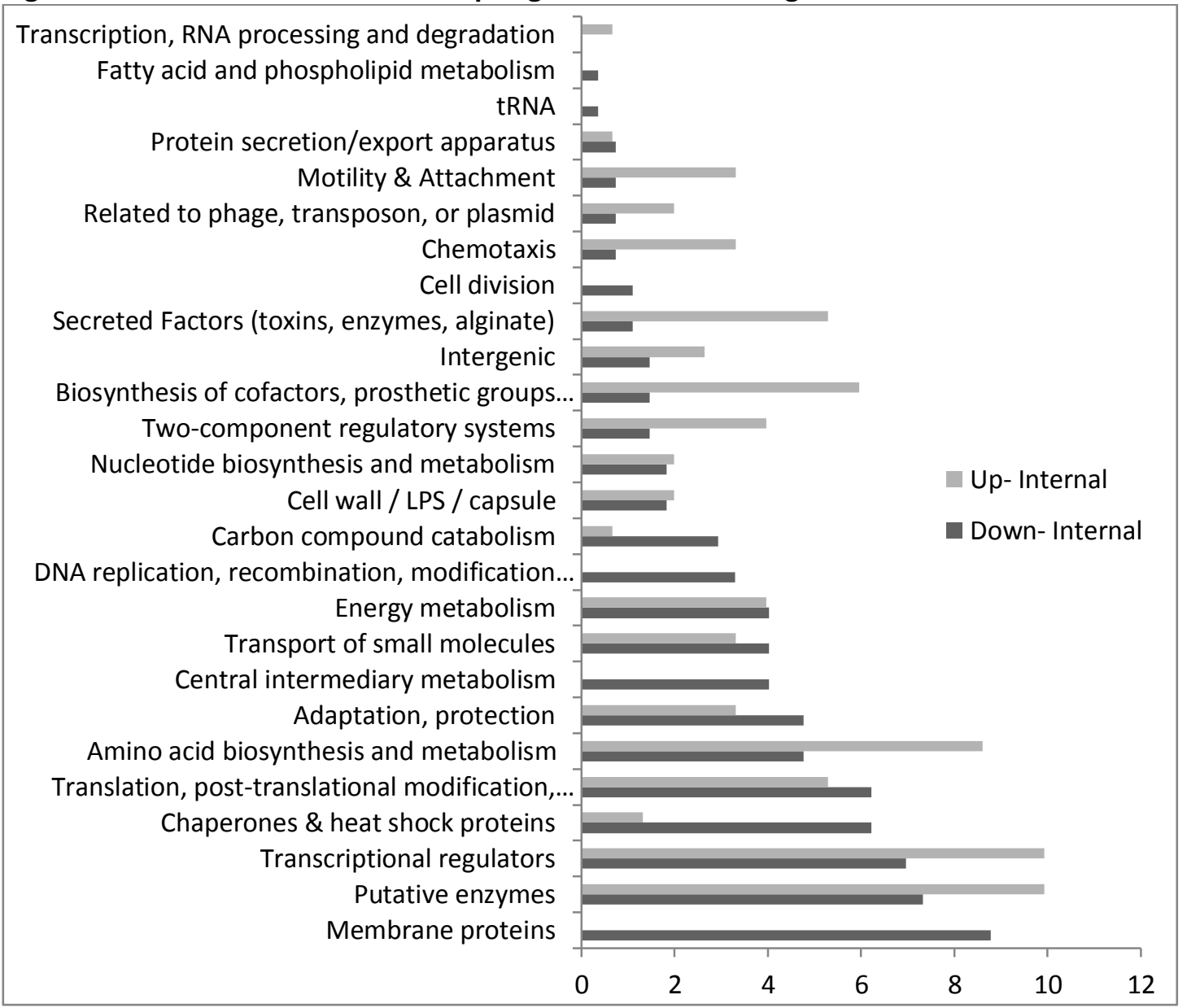


Figure A3.2. Percent External PAO1 up-regulated vs. down-regulated

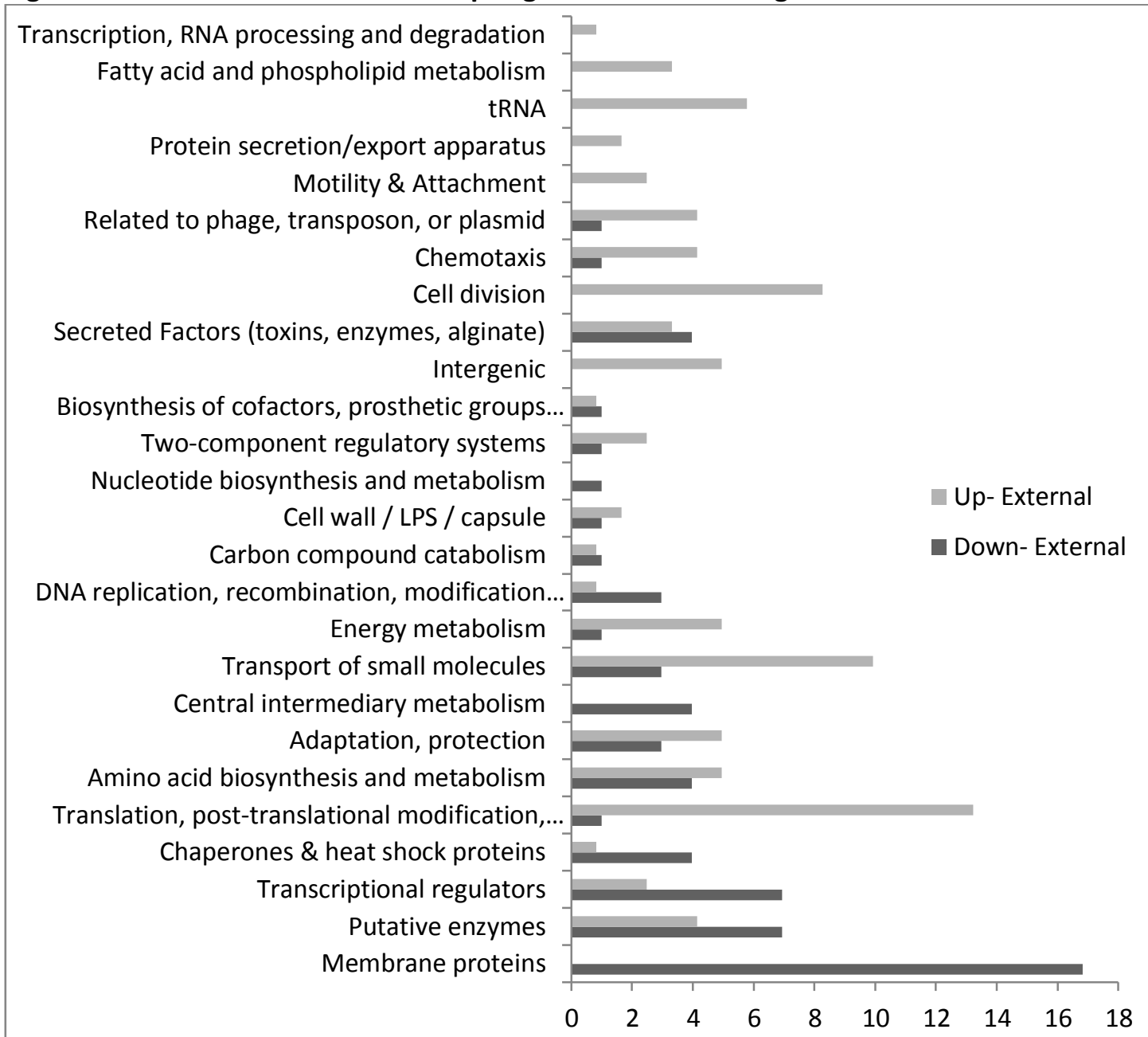


Figure A3.3. Percent Internal PAO1 vs. External PAO1 up-regulated and down-regulated

Transcription, RNA processing and degradation

Fatty acid and phospholipid metabolism

tRNA

Protein secretion/export apparatus

Motility \& Attachment

Related to phage, transposon, or plasmid

Chemotaxis

Cell division

Secreted Factors (toxins, enzymes, alginate)

Intergenic

Biosynthesis of cofactors, prosthetic groups...

Two-component regulatory systems

Nucleotide biosynthesis and metabolism

Cell wall / LPS / capsule

Carbon compound catabolism

DNA replication, recombination, modification.

Energy metabolism

Transport of small molecules

Central intermediary metabolism

Adaptation, protection

Amino acid biosynthesis and metabolism

Translation, post-translational modification,..

Chaperones \& heat shock proteins

Transcriptional regulators

Putative enzymes

Membrane proteins

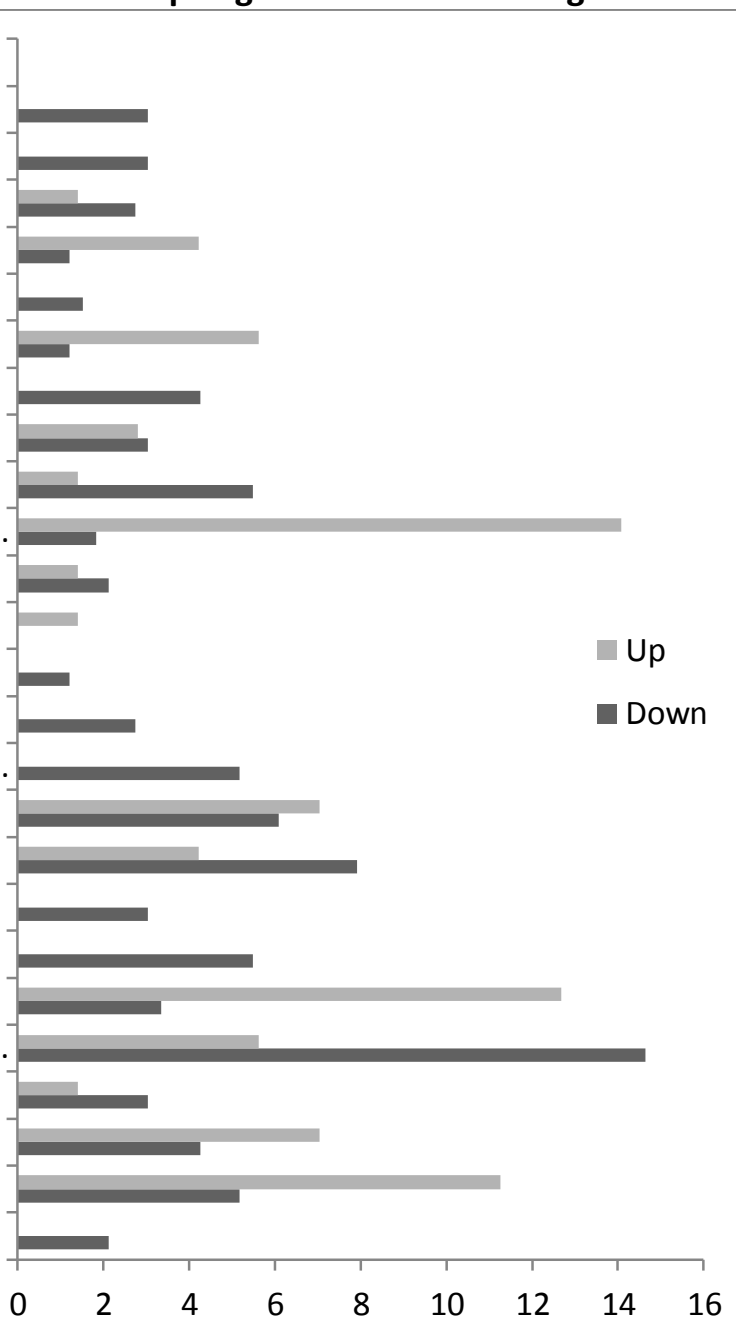


Figure A3.4. Percent up-regulated genes Internal PAO1 vs. External PAO1

Transcription, RNA processing and degradation

Fatty acid and phospholipid metabolism

tRNA

Protein secretion/export apparatus

Motility \& Attachment

Related to phage, transposon, or plasmid

Chemotaxis

Cell division

Secreted Factors (toxins, enzymes, alginate)

Intergenic

Biosynthesis of cofactors, prosthetic groups..

Two-component regulatory systems

Nucleotide biosynthesis and metabolism

Cell wall / LPS / capsule

Carbon compound catabolism

DNA replication, recombination, modification.

Energy metabolism

Transport of small molecules

Central intermediary metabolism

Adaptation, protection

Amino acid biosynthesis and metabolism

Translation, post-translational modification,..

Chaperones \& heat shock proteins

Transcriptional regulators

Putative enzymes

Membrane proteins
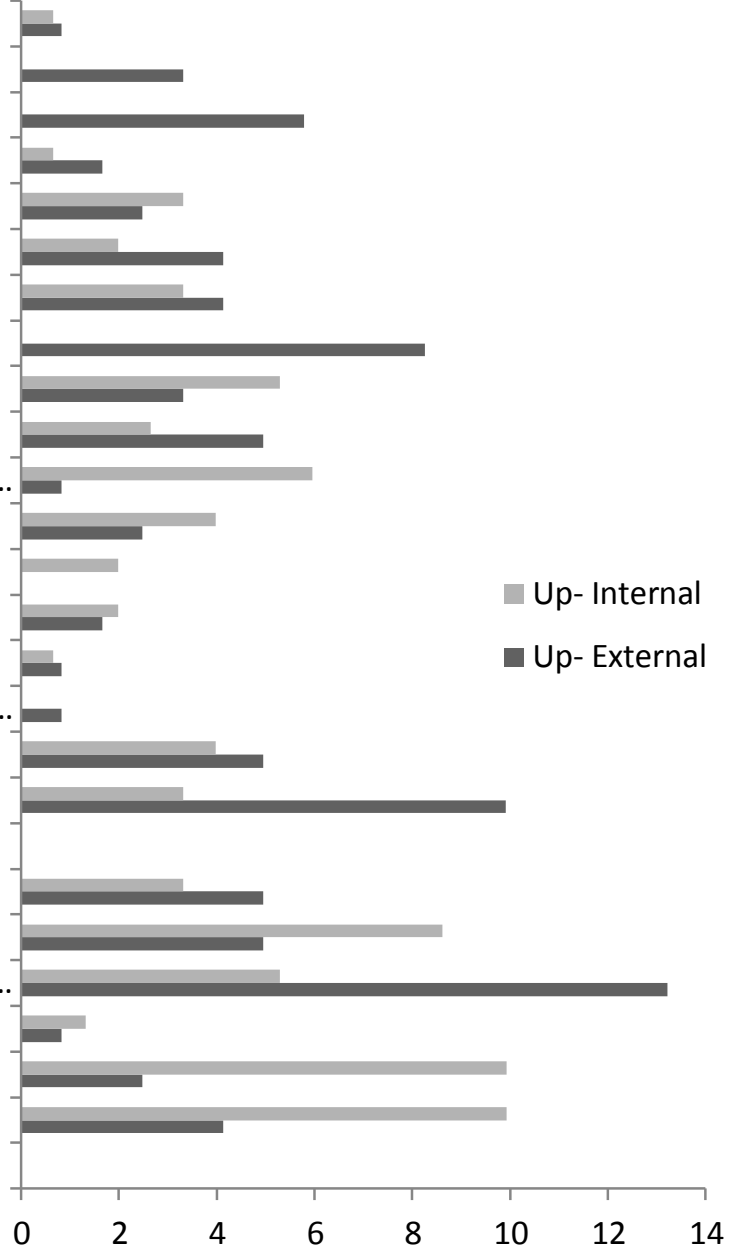
Figure A3.5. Precent down-regulated genes Internal PAO1 vs. External PAO1

Transcription, RNA processing and degradation

Fatty acid and phospholipid metabolism

tRNA

Protein secretion/export apparatus Motility \& Attachment

Related to phage, transposon, or plasmid Chemotaxis

Cell division

Secreted Factors (toxins, enzymes, alginate) Intergenic

Biosynthesis of cofactors, prosthetic groups...

Two-component regulatory systems

Nucleotide biosynthesis and metabolism

Cell wall / LPS / capsule

Carbon compound catabolism

DNA replication, recombination, modification...

Energy metabolism

Transport of small molecules

Central intermediary metabolism

Adaptation, protection

Amino acid biosynthesis and metabolism

Translation, post-translational modification,...

Chaperones \& heat shock proteins

Transcriptional regulators

Putative enzymes

Membrane proteins

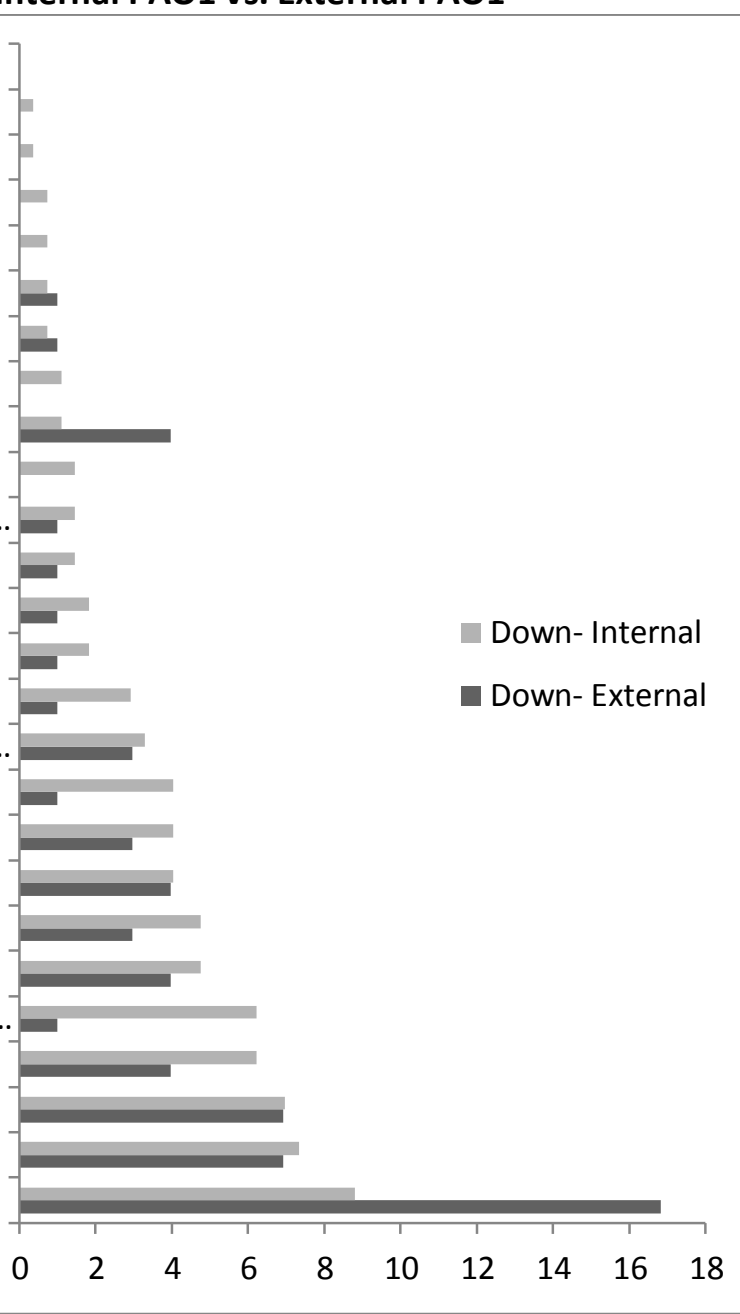

Table A3.1. Percent "Hypothetical, Unclassified, Unknown" genes by data set

\begin{tabular}{|c|c|c|c|c|c|c|}
\hline Direction & Down- & Down- & Down & Up- & Up- & Up \\
\hline Sample & $\begin{array}{c}\text { External }^{a} \\
\text { PAO1 }\end{array}$ & $\begin{array}{c}\text { Internal }^{\mathrm{a}} \\
\text { PAO1 }\end{array}$ & $\begin{array}{c}\text { Internal- } \\
\text { External }^{\text {b }} \\
\text { PAO1 }\end{array}$ & $\begin{array}{c}\text { External }^{\mathrm{a}} \\
\text { PAO1 }\end{array}$ & $\begin{array}{c}\text { Internal }^{\mathrm{a}} \\
\text { PAO1 }\end{array}$ & $\begin{array}{c}\text { Internal- } \\
\text { External }^{b} \\
\text { PAO1 }\end{array}$ \\
\hline$\%$ & 47.52 & 39.19 & 19.82 & 9.92 & 27.81 & 25.35 \\
\hline
\end{tabular}

${ }^{a}$ Internal PAO1 or External PAO1 as compared to Control PAO1 data set.

${ }^{b}$ Internal PAO1 as compared to External PAO1 data set. 


\section{References}

1. National Nosocomial Infections Surveillance (NNIS) System Report, data summary from January 1992 through June 2004, issued October 2004. Am J Infect Control. 2004 Dec;32(8):470-85.

2. Van Delden C, Iglewski BH. Cell-to-cell signalling and Pseudomonas aeruginosa infections. 1998;

3. Stover CK, Pham XQ, Erwin AL, Mizoguchi SD, Warrener P, Hickey MJ, et al. Complete genome sequence of Pseudomonas aeruginosa PAO1, an opportunistic pathogen. Nature. 2000 Aug 31;406(6799):959-64.

4. Scott R. The direct medical costs of healthcare-associated infections in U.S. hospitals and the benefits of prevention [Internet]. Centers for Disease Control and Prevention. 2009. Available from:

http://www.cdc.gov/hai/pdfs/hai/scott_costpaper.pdf

5. CDC - NHSN [Internet]. [cited 2011 Nov 27]. Available from: http://www.cdc.gov/nhsn/

6. International Federation of Infection Control. The Costs of Hospital Infection [Internet]. Available from: http://www.ific.narod.ru/Manual/cost.htm

7. Lynch JP. Hospital-acquired pneumonia: risk factors, microbiology, and treatment. Chest. 2001;119(2 Suppl):373S-384S.

8. Davis PB, Di Sant'Agnese PA. Assisted Ventilation for Patients With Cystic Fibrosis. JAMA. 1978 May 5;239(18):1851-4.

9. Kremer TM, Zwerdling RG, Michelson PH, O'Sullivan P. Intensive care management of the patient with cystic fibrosis. J Intensive Care Med. 2008 Jun;23(3):159-77.

10. Rello J, Ollendorf DA, Oster G, Vera-Llonch M, Bellm L, Redman R, et al. Epidemiology and Outcomes of Ventilator-Associated Pneumonia in a Large US Database*. Chest. 2002 Jan 12;122(6):2115-21.

11. Fujitani S, Sun H-Y, Yu VL, Weingarten JA. Pneumonia Due to Pseudomonas Aeruginosa Part I: Epidemiology, Clinical Diagnosis, and Source. Chest. 2011 Jan 4;139(4):909-19.

12. Murray TS, Egan M, Kazmierczak BI. Pseudomonas aeruginosa chronic colonization in cystic fibrosis patients. Curr Opin Pediatr. 2007;19(1):83-8.

13. Chastre J, Fagon J-Y. Ventilator-Associated Pneumonia. Am. J. Respir. Crit. Care Med. 2002 Jan 4;165(7):867-903. 
14. Flanagan JL, Brodie EL, Weng L, Lynch SV, Garcia O, Brown R, et al. Loss of Bacterial Diversity During Antibiotic Treatment of Intubated Patients Colonized with Pseudomonas Aeruginosa. J. Clin. Microbiol. 2007 Jan 6;45(6):1954-62.

15. McGowan JE. Resistance in nonfermenting gram-negative bacteria: multidrug resistance to the maximum. Am J Med. 2006;119(6 Suppl 1):S29-36; discussion S62-70.

16. Parham P. The Immune System. Garland Publishing/Elseiver Science Ltd.; 2000.

17. Mishra M, Byrd MS, Sergeant S, Azad AK, Parsek MR, McPhail L, et al. Pseudomonas aeruginosa Psl polysaccharide reduces neutrophil phagocytosis and the oxidative response by limiting complement-mediated opsonization. Cellular Microbiology. 2012 Jan 1;14(1):95-106.

18. Todar K. Pseudomonas aeruginosa. Todar's Online Textbook of Bacteriology. 2004;

19. Eisele NA, Anderson DM. Host Defense and the Airway Epithelium: Frontline Responses That Protect against Bacterial Invasion and Pneumonia. Journal of Pathogens. 2011;2011:1-16.

20. Davies JA, Garrod DR. Molecular aspects of the epithelial phenotype. BioEssays. 1997;19(8):699-704.

21. Lee A, Chow D, Haus B, Tseng W, Evans D, Fleiszig S, et al. Airway epithelial tight junctions and binding and cytotoxicity of Pseudomonas aeruginosa. American Journal of Physiology - Lung Cellular and Molecular Physiology. 1999 Jul 1;277(1):L204-L217.

22. Arnold RR, Cole MF, McGhee. A Bactericidal Effect for Human Lactoferrin. Science. 1977 Jul 15;197(4300):263-5.

23. Reiter B, Brock JH, Steel ED. Inhibition of Escherichia coli by bovine colostrum and post-colostral milk. II. The bacteriostatic effect of lactoferrin on a serum susceptible and serum resistant strain of E. coli. Immunology. 1975 Jan;28(1):83-95.

24. Ellison RT, Giehl TJ, LaForce FM. Damage of the Outer Membrane of Enteric Gram-Negative Bacteria by Lactoferrin and Transferrin. Infect. Immun. 1988 Jan $11 ; 56(11): 2774-81$.

25. Kinnula VL, Adler KB, Ackley NJ, Crapo JD. Release of reactive oxygen species by guinea pig tracheal epithelial cells in vitro. Am. J. Physiol. 1992 Jun;262(6 Pt 1):L708-712.

26. Yang $\mathrm{F}$, Jin $\mathrm{S}, \mathrm{Xu} \mathrm{Y}$, Lu Y. Comparisons of IL-8, ROS and $\mathrm{p} 53$ responses in human lung epithelial cells exposed to two extracts of PM2.5 collected from an e-waste recycling area, China. Environmental Research Letters. 2011 Apr 1;6(2):024013. 
27. Boots AW, Hristova M, Kasahara DI, Haenen GRMM, Bast A, van der Vliet A. ATPmediated activation of the NADPH oxidase DUOX1 mediates airway epithelial responses to bacterial stimuli. J. Biol. Chem. 2009 Jun 26;284(26):17858-67.

28. Harder J, Meyer-Hoffert U, Teran LM, Schwichtenberg L, Bartels J, Maune S, et al. Mucoid Pseudomonas aeruginosa, TNF-alpha, and IL-1beta, but not IL-6, induce human beta-defensin-2 in respiratory epithelia. Am. J. Respir. Cell Mol. Biol. 2000 Jun;22(6):714-21.

29. Suntres ZE, Omri A, Shek PN. Pseudomonas aeruginosa-induced lung injury: role of oxidative stress. Microb. Pathog. 2002 Jan;32(1):27-34.

30. Walport MJ. Complement. First of two parts. N. Engl. J. Med. 2001 Apr 5;344(14):1058-66.

31. Underhill DM, Ozinsky A. PHAGOCYTOSIS OF MICROBES : Complexity in Action. Annual Review of Immunology. 2002 Apr;20:825-52.

32. Aderem A, Underhill DM. Mechanisms of Phagocytosis in Macrophages. Annual Review of Immunology. 1999;17(1):593-623.

33. Peiser L, Gordon S. Phagocytosis: Enhancement. eLS [Internet]. John Wiley \& Sons, Ltd; 2001 [cited 2012 Jun 10]. Available from:

http://onlinelibrary.wiley.com/doi/10.1002/9780470015902.a0001214.pub2/ab stract

34. Jucker BA, Harms H, Zehnder AJ. Adhesion of the Positively Charged Bacterium Stenotrophomonas (Xanthomonas) Maltophilia 70401 to Glass and Teflon. J. Bacteriol. 1996 Jan 9;178(18):5472-9.

35. van Oss CJ, Absolom DR, Neumann AW. Interaction of Phagocytes with Other Blood Cells and with Pathogenic and Nonpathogenic Microbes. Annals of the New York Academy of Sciences. 1983;416(1):332-50.

36. Hirsch JG, Strauss B. Studies on Heat-Labile Opsonin in Rabbit Serum. J Immunol. 1964 Jan 1;92(1):145-54.

37. Peterson PK, Gekker G, Shapiro R, Freiberg M, Keane WF. Polyamino Acid Enhancement of Bacterial Phagocytosis by Human Polymorphonuclear Leukocytes and Peritoneal Macrophages. Infect. Immun. 1984 Jan 2;43(2):5616.

38. Weiss J, Victor M, Elsbach P. Role of Charge and Hydrophobic Interactions in the Action of Bactericidal/Permeability-increasing Protein of Neutrophils on Gramnegative Bacteria. J Clin Invest. 1983 Mar;71(3):540-9.

39. Guan E, Robinson SL, Goodman EB, Tenner AJ. Cell-Surface Protein Identified on Phagocytic Cells Modulates the C1q-Mediated Enhancement of Phagocytosis. J Immunol. 1994 Apr 15;152(8):4005-16. 
40. Sadikot RT, Blackwell TS, Christman JW, Prince AS. Pathogen-host interactions in Pseudomonas aeruginosa pneumonia. Am. J. Respir. Crit. Care Med. 2005 Jun 1;171(11):1209-23.

41. Feldman M, Bryan R, Rajan S, Scheffler L, Brunnert S, Tang H, et al. Role of Flagella in Pathogenesis of Pseudomonas aeruginosa Pulmonary Infection. Infect Immun. 1998 Jan;66(1):43-51.

42. Chi E, Mehl T, Nunn D, Lory S. Interaction of Pseudomonas aeruginosa with A549 pneumocyte cells. Infection and Immunity. 1991 Mar 1;59(3):822 -828.

43. Soong G, Reddy B, Sokol S, Adamo R, Prince A. TLR2 is mobilized into an apical lipid raft receptor complex to signal infection in airway epithelial cells. J Clin Invest. 2004 May 15;113(10):1482-9.

44. Hauser AR, Fleiszig S, Kang PJ, Mostov K, Engel JN. Defects in Type III Secretion Correlate with Internalization of Pseudomonas aeruginosa by Epithelial Cells. Infect Immun. 1998 Apr;66(4):1413-20.

45. Culver DA, Barna BP, Raychaudhuri B, Bonfield TL, Abraham S, Malur A, et al. Peroxisome proliferator-activated receptor gamma activity is deficient in alveolar macrophages in pulmonary sarcoidosis. Am. J. Respir. Cell Mol. Biol. 2004 Jan;30(1):1-5.

46. Kipnis E, Sawa T, Wiener-Kronish J. Targeting mechanisms of Pseudomonas aeruginosa pathogenesis. Med Mal Infect. 2006 Feb;36(2):78-91.

47. Azghani AO, Connelly JC, Peterson BT, Gray LD, Collins ML, Johnson AR. Effects of Pseudomonas aeruginosa elastase on alveolar epithelial permeability in guinea pigs. Infect Immun. 1990 Feb;58(2):433-8.

48. Azghani AO, Gray LD, Johnson AR. A bacterial protease perturbs the paracellular barrier function of transporting epithelial monolayers in culture. Infect Immun. 1993 Jun;61(6):2681-6.

49. Tziortzioti V, Petropoulou H, Tsaganos T, Spyridaki A, Raftogiannis M, Giamarellos-Bourboulis EJ, et al. Skin Biopsy is Predictive of Outcome in Experimental Sepsis by Multidrug- Resistant Pseudomonas aeruginosa. The Open Dermatology Journal. 2007;(1):1-5.

50. Seeger W, Walmrath D, Neuhof H, Lutz F. Pulmonary Microvascular Injury Induced by Pseudomonas Aeruginosa Cytotoxin in Isolated Rabbit Lungs. Infect. Immun. 1986 Jan 6;52(3):846-52.

51. Bragonzi $A$, Worlitzsch $D$, Pier GB, Timpert $P$, Ulrich $M$, Hentzer $M$, et al. Nonmucoid Pseudomonas aeruginosa expresses alginate in the lungs of patients with cystic fibrosis and in a mouse model. J. Infect. Dis. 2005 Aug 1;192(3):4109. 
52. Govan JR, Deretic V. Microbial pathogenesis in cystic fibrosis: mucoid Pseudomonas aeruginosa and Burkholderia cepacia. Microbiol Rev. 1996 Sep;60(3):539-74.

53. Oliver AM, Weir DM. The effect of Pseudomonas alginate on rat alveolar macrophage phagocytosis and bacterial opsonization. Clin Exp Immunol. 1985 Jan;59(1):190-6.

54. Tang S, Leung JCK, Abe K, Chan KW, Chan LYY, Chan TM, et al. Albumin stimulates interleukin-8 expression in proximal tubular epithelial cells in vitro and in vivo. J Clin Invest. 2003 Feb 15;111(4):515-27.

55. Ghafoor A, Hay ID, Rehm BHA. Role of Exopolysaccharides in Pseudomonas aeruginosa Biofilm Formation and Architecture $\nabla$. Appl Environ Microbiol. 2011 Aug;77(15):5238-46.

56. Nivens DE, Ohman DE, Williams J, Franklin MJ. Role of alginate and its $\mathrm{O}$ acetylation in formation of Pseudomonas aeruginosa microcolonies and biofilms. J. Bacteriol. 2001 Feb;183(3):1047-57.

57. Pier GB, Coleman F, Grout M, Franklin M, Ohman DE. Role of alginate $\mathrm{O}$ acetylation in resistance of mucoid Pseudomonas aeruginosa to opsonic phagocytosis. Infect. Immun. 2001 Mar;69(3):1895-901.

58. Hogardt M, Heesemann J. Adaptation of Pseudomonas aeruginosa during persistence in the cystic fibrosis lung. International Journal of Medical Microbiology. 2010 Dec;300(8):557-62.

59. Babior BM. Activation of the respiratory burst oxidase. Environ Health Perspect. 1994;102 Suppl 10:53-6.

60. Patterson-Delafield J, Martinez RJ, Lehrer RI. Microbicidal cationic proteins in rabbit alveolar macrophages: a potential host defense mechanism. Infect. Immun. 1980 Oct;30(1):180-92.

61. Spitznagel JK. Nonoxidative antimicrobial reactions of leukocytes. Contemp Top Immunobiol. 1984;14:283-343.

62. Babior BM. Superoxide: a two-edged sword. Braz. J. Med. Biol. Res. 1997 Feb;30(2):141-55.

63. Babior BM. The respiratory burst of phagocytes. J Clin Invest. 1984 Mar;73(3):599-601.

64. Folkes LK, Candeias LP, Wardman P. Kinetics and mechanisms of hypochlorous acid reactions. Arch. Biochem. Biophys. 1995 Oct 20;323(1):120-6.

65. Schraufstätter IU, Browne K, Harris A, Hyslop PA, Jackson JH, Quehenberger O, et al. Mechanisms of hypochlorite injury of target cells. J. Clin. Invest. 1990 Feb;85(2):554-62. 
66. Klebanoff S. Phagocytic cells: products of oxygen metabolism. Inflammation: basic principles and clinical correlates [Internet]. New York: Raven Press; 1988 [cited 2012 May 8]. p. 391-443. Available from: http://www.springerlink.com/content/p72u74867px66008/abstract/

67. Rossi F, Zabucchi G, Dri P, Bellavite $\mathrm{P}$, Berton G. O2- and $\mathrm{H} 2 \mathrm{O} 2$ production during the respiratory burst in alveolar macrophages. Adv. Exp. Med. Biol. 1979;121(A):53-74.

68. Breen AP, Murphy JA. Reactions of oxyl radicals with DNA. Free Radic. Biol. Med. 1995 Jun;18(6):1033-77.

69. von Sonntag $C$. New aspects in the free-radical chemistry of pyrimidine nucleobases. Free Radic. Res. Commun. 1987;2(4-6):217-24.

70. Halliwell B, Gutteridge JM, editors. Free Radicals in Biology and Medicine. 3rd ed. New York: Oxford University Press; 1999.

71. Altman SA, Zastawny TH, Randers-Eichhorn L, Cacciuttolo MA, Akman SA, Dizdaroglu $\mathrm{M}$, et al. Formation of DNA-protein cross-links in cultured mammalian cells upon treatment with iron ions. Free Radic. Biol. Med. 1995 Dec;19(6):897-902.

72. Mollnes TE, Brekke O-L, Fung M, Fure H, Christiansen D, Bergseth G, et al. Essential role of the $\mathrm{C} 5 \mathrm{a}$ receptor in $\mathrm{E}$ coli-induced oxidative burst and phagocytosis revealed by a novel lepirudin-based human whole blood model of inflammation [Internet]. [cited 2012 Jun 30]. Available from: http://bloodjournal.hematologylibrary.org

73. Villegas J, Schulz M, Soto L, Sanchez R. Bacteria induce expression of apoptosis in human spermatozoa. Apoptosis. 2005;10(1):105-10.

74. Hyslop PA, Hinshaw DB, Scraufstatter IU, Cochrane CG, Kunz S, Vosbeck K. Hydrogen peroxide as a potent bacteriostatic antibiotic: implications for host defense. Free Radic Biol Med. 1995;19(1):31-7.

75. Sies H. Oxidative Stress. 1985.

76. Babior BM. Neutrophil function as related to neutrophil-endothelial cell interactions. Nouv Rev Fr Hematol. 1992;34 Suppl:S29-35.

77. Belousov VV, Fradkov AF, Lukyanov KA, Staroverov DB, Shakhbazov KS, Terskikh $A V$, et al. Genetically encoded fluorescent indicator for intracellular hydrogen peroxide. Nat Methods. 2006;3(4):281-6.

78. Salunkhe P, Topfer T, Buer J, Tummler B. Genome-Wide Transcriptional Profiling of the Steady-State Response of Pseudomonas aeruginosa to Hydrogen Peroxide. J. Bacteriol. 2005 Apr 15;187(8):2565-72. 
79. Neumann M, Kownatzki E. The effect of adherence on the generation of reactive oxygen species by human neutrophilic granulocytes. Agents Actions. 1989 Jan;26(1-2):183-5.

80. Demple B. A bridge to control. Science. 1998 Mar 13;279(5357):1655-6.

81. Barja G. Oxygen radicals, a failure or a success of evolution? Free Radic. Res. Commun. 1993;18(2):63-70.

82. Matz C. Biofilms as Refuge against Predation. The Biofilm Mode of Life: Mechanisms and Adaptation [Internet]. Horizon Bioscience; 2007. Available from: http://www.open-access-biology.com/biofilms/biofilmsch11.pdf

83. Twining SS, Kirschner SE, Mahnke LA, Frank DW. Effect of Pseudomonas aeruginosa elastase, alkaline protease, and exotoxin $A$ on corneal proteinases and proteins. Invest. Ophthalmol. Vis. Sci. 1993 Aug;34(9):2699-712.

84. Vabulas RM, Raychaudhuri S, Hayer-Hartl M, Hartl FU. Protein Folding in the Cytoplasm and the Heat Shock Response. Cold Spring Harb Perspect Biol [Internet]. 2010 Jan 12 [cited 2012 Jun 10];2(12). Available from: http://cshperspectives.cshlp.org/content/2/12/a004390

85. Shinnick TM. Heat shock proteins as antigens of bacterial and parasitic pathogens. Curr. Top. Microbiol. Immunol. 1991;167:145-60.

86. Steinman L. Escape from 'horror autotoxicus': pathogenesis and treatment of autoimmune disease. Cell. 1995 Jan 13;80(1):7-10.

87. Theofilopoulos AN. The basis of autoimmunity: Part I. Mechanisms of aberrant self-recognition. Immunol. Today. 1995 Feb;16(2):90-8.

88. Wand-Württenberger A, Schoel B, Ivanyi J, Kaufmann SH. Surface expression by mononuclear phagocytes of an epitope shared with mycobacterial heat shock protein 60. Eur. J. Immunol. 1991 Apr;21(4):1089-92.

89. Kaufmann SH, Schoel B, van Embden JD, Koga T, Wand-Württenberger A, Munk $\mathrm{ME}$, et al. Heat-shock protein 60: implications for pathogenesis of and protection against bacterial infections. Immunol. Rev. 1991 Jun;121:67-90.

90. O'Malley YQ, Reszka KJ, Rasmussen GT, Abdalla MY, Denning GM, Britigan BE. The Pseudomonas secretory product pyocyanin inhibits catalase activity in human lung epithelial cells. Am. J. Physiol. Lung Cell Mol. Physiol. 2003 Nov;285(5):L1077-1086.

91. O’Malley YQ, Abdalla MY, McCormick ML, Reszka KJ, Denning GM, Britigan BE. Subcellular localization of Pseudomonas pyocyanin cytotoxicity in human lung epithelial cells. Am. J. Physiol. Lung Cell Mol. Physiol. 2003 Feb;284(2):L420-430.

92. Usher LR, Lawson RA, Geary I, Taylor CJ, Bingle CD, Taylor GW, et al. Induction of neutrophil apoptosis by the Pseudomonas aeruginosa exotoxin pyocyanin: a 
potential mechanism of persistent infection. J. Immunol. 2002 Feb 15;168(4):1861-8.

93. Tateda K, Ishii Y, Horikawa M, Matsumoto T, Miyairi S, Pechere JC, et al. The Pseudomonas aeruginosa Autoinducer N-3-Oxododecanoyl Homoserine Lactone Accelerates Apoptosis in Macrophages and Neutrophils. Infect Immun. 2003 Oct;71(10):5785-93.

94. Baron SS, Rowe JJ. Antibiotic Action of Pyocyanin. Antimicrob. Agents Chemother. 1981 Jan 12;20(6):814-20.

95. Sano $Y$, Matsui $H$, Kobayashi M, Kageyama M. Molecular structures and functions of pyocins S1 and S2 in Pseudomonas aeruginosa. J. Bacteriol. 1993 May;175(10):2907-16.

96. Sano $\mathrm{Y}$, Kageyama M. A novel transposon-like structure carries the genes for pyocin AP41, a Pseudomonas aeruginosa bacteriocin with a DNase domain homology to E2 group colicins. Mol. Gen. Genet. 1993 Feb;237(1-2):161-70.

97. Firoved AM, Deretic V. Microarray Analysis of Global Gene Expression in Mucoid Pseudomonas aeruginosa. J. Bacteriol. 2003 Feb 1;185(3):1071-81.

98. Firoved AM, Ornatowski W, Deretic V. Microarray analysis reveals induction of lipoprotein genes in mucoid Pseudomonas aeruginosa: implications for inflammation in cystic fibrosis. Infect. Immun. 2004 Sep;72(9):5012-8.

99. Mathee K, Ciofu O, Sternberg C, Lindum PW, Campbell JI, Jensen P, et al. Mucoid conversion of Pseudomonas aeruginosa by hydrogen peroxide: a mechanism for virulence activation in the cystic fibrosis lung. Microbiology (Reading, Engl.). 1999 Jun;145 ( Pt 6):1349-57.

100. Learn DB, Brestel EP, Seetharama S. Hypochlorite scavenging by Pseudomonas aeruginosa alginate. Infect. Immun. 1987 Aug;55(8):1813-8.

101. Fridovich I. The biology of oxygen radicals. Science. 1978 Sep 8;201(4359):87580.

102. Gregory EM, Fridovich I. Induction of superoxide dismutase by molecular oxygen. J. Bacteriol. 1973 May;114(2):543-8.

103. Fridovich I. Superoxide radical and superoxide dismutases. Annu. Rev. Biochem. 1995;64:97-112.

104. Hassett DJ, Charniga L, Bean K, Ohman DE, Cohen MS. Response of Pseudomonas Aeruginosa to Pyocyanin: Mechanisms of Resistance, Antioxidant Defenses, and Demonstration of a Manganese-Cofactored Superoxide Dismutase. Infect. Immun. 1992 Jan 2;60(2):328-36.

105. Hassett DJ, Woodruff WA, Wozniak DJ, Vasil ML, Cohen MS, Ohman DE. Cloning and characterization of the Pseudomonas aeruginosa sod $A$ and sodB genes encoding manganese- and iron-cofactored superoxide dismutase: 
demonstration of increased manganese superoxide dismutase activity in alginate-producing bacteria. J. Bacteriol. 1993 Dec;175(23):7658-65.

106. Polack B, Dacheux D, Delic-Attree I, Toussaint B, Vignais PM. Role of manganese superoxide dismutase in a mucoid isolate of Pseudomonas aeruginosa: adaptation to oxidative stress. Infect. Immun. 1996 Jun;64(6):2216-9.

107. Britigan BE, Miller RA, Hassett DJ, Pfaller MA, McCormick ML, Rasmussen GT. Antioxidant enzyme expression in clinical isolates of Pseudomonas aeruginosa: identification of an atypical form of manganese superoxide dismutase. Infect. Immun. 2001 Dec;69(12):7396-401.

108. Hassett DJ, Schweizer HP, Ohman DE. Pseudomonas aeruginosa sodA and sodB mutants defective in manganese- and iron-cofactored superoxide dismutase activity demonstrate the importance of the iron-cofactored form in aerobic metabolism. J Bacteriol. 1995 Nov;177(22):6330-7.

109. Brown SM, Howell ML, Vasil ML, Anderson AJ, Hassett DJ. Cloning and characterization of the katB gene of Pseudomonas aeruginosa encoding a hydrogen peroxide-inducible catalase: purification of KatB, cellular localization, and demonstration that it is essential for optimal resistance to hydrogen peroxide. J Bacteriol. 1995 Nov;177(22):6536-44.

110. Ma J-F, Ochsner UA, Klotz MG, Nanayakkara VK, Howell ML, Johnson Z, et al. Bacterioferritin A Modulates Catalase A (KatA) Activity and Resistance to Hydrogen Peroxide in Pseudomonas Aeruginosa. J. Bacteriol. 1999 Jun 15;181(12):3730-42.

111. Lee JS, Heo YJ, Lee JK, Cho YH. KatA, the major catalase, is critical for osmoprotection and virulence in Pseudomonas aeruginosa PA14. Infect Immun. 2005;73(7):4399-403.

112. Shin D-H, Choi Y-S, Cho Y-H. Unusual Properties of Catalase A (KatA) of Pseudomonas aeruginosa PA14 Are Associated with Its Biofilm Peroxide Resistance. J Bacteriol. 2008 Apr;190(8):2663-70.

113. Elkins JG, Hassett DJ, Stewart PS, Schweizer HP, McDermott TR. Protective Role of Catalase in Pseudomonas Aeruginosa Biofilm Resistance to Hydrogen Peroxide. Appl. Environ. Microbiol. 1999 Jan 10;65(10):4594-600.

114. Scandalios JG. Oxidative stress: molecular perception and transduction of signals triggering antioxidant gene defenses. Brazilian Journal of Medical and Biological Research. 2005 Jul;38(7):995-1014.

115. Cadenas E, Hochstein P, Ernster L. Pro- and antioxidant functions of quinones and quinone reductases in mammalian cells. Adv. Enzymol. Relat. Areas Mol. Biol. 1992;65:97-146.

116. Lin AJ, Cosby LA, Shansky CW, Sartorelli AC. Potential bioreductive alkylating agents. 1. Benzoquinone derivatives. J. Med. Chem. 1972 Dec;15(12):1247-52. 
117. Siegel D, Bolton EM, Burr JA, Liebler DC, Ross D. The reduction of alphatocopherolquinone by human $\mathrm{NAD}(\mathrm{P}) \mathrm{H}$ : quinone oxidoreductase: the role of alpha-tocopherolhydroquinone as a cellular antioxidant. Mol Pharmacol. 1997;52(2):300-5.

118. Carey J, Brynda J, Wolfová J, Grandori R, Gustavsson T, Ettrich R, et al. WrbA bridges bacterial flavodoxins and eukaryotic NAD(P)H:quinone oxidoreductases. Protein Sci. 2007 Oct;16(10):2301-5.

119. Liochev $\mathrm{SI}$, Hausladen A, Fridovich I. Nitroreductase A is regulated as a member of the soxRS regulon of Escherichia coli. PNAS. 1999 Mar 30;96(7):3537-9.

120. Ernster L, Navazio R. Soluble diaphorase in animal tissues. Acta Chem Scand. 1958;(12):595.

121. Siegel D, Gustafson DL, Dehn DL, Han JY, Boonchoong P, Berliner L, et al. $\mathrm{NAD}(\mathrm{P}) \mathrm{H}$ :quinone oxidoreductase 1: role as a superoxide scavenger. Mol Pharmacol. 2004;65(5):1238-47.

122. Cadenas E. Antioxidant and prooxidant functions of DT-diaphorase in quinone metabolism. Biochem Pharmacol. 1995;49(2):127-40.

123. Lind C, Cadenas E, Hochstein P, Ernster L. DT-diaphorase: purification, properties, and function. Methods Enzymol. 1990;186:287-301.

124. Yang W, Ni L, Somerville RL. A stationary-phase protein of Escherichia coli that affects the mode of association between the trp repressor protein and operatorbearing DNA. Proc. Natl. Acad. Sci. U.S.A. 1993 Jun 15;90(12):5796-800.

125. Grandori R, Carey J. Six new candidate members of the $\alpha / \beta$ twisted open-sheet family detected by sequence similarity to flavodoxin. Protein Science. 1994;3(12):2185-93.

126. Persson B, Zigler JS, Jörnvall H. A Super-Family of Medium-Chain Dehydrogenases/Reductases (MDR). European Journal of Biochemistry. 1994;226(1):15-22.

127. Ackerley DF, Gonzalez CF, Park CH, Blake R 2nd, Keyhan M, Matin A. Chromatereducing properties of soluble flavoproteins from Pseudomonas putida and Escherichia coli. Appl. Environ. Microbiol. 2004 Feb;70(2):873-82.

128. Wang G, Maier RJ. An NADPH quinone reductase of Helicobacter pylori plays an important role in oxidative stress resistance and host colonization. Infect Immun. 2004;72(3):1391-6.

129. Adams MA, Jia Z. Structural and biochemical evidence for an enzymatic quinone redox cycle in Escherichia coli: identification of a novel quinol monooxygenase. J Biol Chem. 2005;280(9):8358-63. 
130. Gonzalez CF, Ackerley DF, Lynch SV, Matin A. ChrR, a soluble quinone reductase of Pseudomonas putida that defends against H2O2. J Biol Chem.

2005;280(24):22590-5.

131. Akhtar P, Srivastava S, Srivastava A, Srivastava M, Srivastava BS, Srivastava R. Rv3303c of Mycobacterium tuberculosis protects tubercle bacilli against oxidative stress in vivo and contributes to virulence in mice. Microbes Infect. 2006;8(14-15):2855-62.

132. Patridge EV, Ferry JG. WrbA from Escherichia coli and Archaeoglobus fulgidus is an NAD(P)H:quinone oxidoreductase. J Bacteriol. 2006;188(10):3498-506.

133. Guina T, Radulovic D, Bahrami AJ, Bolton DL, Rohmer L, Jones-Isaac KA, et al. MgIA regulates Francisella tularensis subsp. novicida (Francisella novicida) response to starvation and oxidative stress. J Bacteriol. 2007;189(18):6580-6.

134. Lind C, Hochstein P, Ernster L. DT-diaphorase as a quinone reductase: a cellular control device against semiquinone and superoxide radical formation. Arch Biochem Biophys. 1982;216(1):178-85.

135. Hajos AKD, Winston GW. Dinitropyrene nitroreductase activity of purified $\mathrm{NAD}(\mathrm{P}) \mathrm{H}$-quinone oxidoreductase: Role in rat liver cytosol and induction by Aroclor-1254 pretreatment. Carcinogenesis. 1991;12(4):697-702.

136. Anlezark GM, Melton RG, Sherwood RF, Coles B, Friedlos F, Knox RJ. The bioactivation of 5-(aziridin-1-yl)-2,4-dinitrobenzamide (CB1954)--I. Purification and properties of a nitroreductase enzyme from Escherichia coli--a potential enzyme for antibody-directed enzyme prodrug therapy (ADEPT). Biochem. Pharmacol. 1992 Dec 15;44(12):2289-95.

137. Barak Y, Thorne SH, Ackerley DF, Lynch SV, Contag CH, Matin A. New enzyme for reductive cancer chemotherapy, YieF, and its improvement by directed evolution. Mol. Cancer Ther. 2006 Jan;5(1):97-103.

138. Vass SO, Jarrom D, Wilson WR, Hyde El, Searle PF. E. coli NfsA: an alternative nitroreductase for prodrug activation gene therapy in combination with CB1954. Br J Cancer. 2009 Jun 16;100(12):1903-11.

139. Prosser GA, Copp JN, Syddall SP, Williams EM, Smaill JB, Wilson WR, et al. Discovery and evaluation of Escherichia coli nitroreductases that activate the anti-cancer prodrug CB1954. Biochemical Pharmacology. 2010 Mar 1;79(5):67887.

140. Denny WA. Prodrug strategies in cancer therapy. European Journal of Medicinal Chemistry. 2001 Aug;36(7-8):577-95.

141. Knox RJ, Friedlos F, Jarman M, Roberts JJ. A new cytotoxic, DNA interstrand crosslinking agent, 5-(aziridin-1-yl)-4-hydroxylamino-2-nitrobenzamide, is formed from 5-(aziridin-1-yl)-2,4-dinitrobenzamide (CB 1954) by a nitroreductase enzyme in Walker carcinoma cells. Biochem. Pharmacol. 1988 Dec 15;37(24):4661-9. 
142. Friedlos F, Quinn J, Knox RJ, Roberts JJ. The properties of total adducts and interstrand crosslinks in the DNA of cells treated with CB 1954. Exceptional frequency and stability of the crosslink. Biochem. Pharmacol. $1992 \mathrm{Mar}$ $17 ; 43(6): 1249-54$.

143. Ross D, Kepa JK, Winski SL, Beall HD, Anwar A, Siegel D. NAD(P)H:quinone oxidoreductase 1 (NQO1): chemoprotection, bioactivation, gene regulation and genetic polymorphisms. Chemico-Biological Interactions. 2000 Dec 1;129(12):77-97.

144. Springer CJ, Niculescu-Duvaz I. Approaches to Gene-Directed Enzyme Prodrug Therapy (GDEPT). In: Habib NA, editor. Cancer Gene Therapy [Internet]. Springer US; 2002 [cited 2012 Jun 12]. p. 403-9. Available from: http://www.springerlink.com/content/g2705r8p7578954w/abstract/

145. Xu G, McLeod HL. Strategies for Enzyme/Prodrug Cancer Therapy. Clin Cancer Res. 2001 Jan 11;7(11):3314-24.

146. Denny WA. Nitroreductase-based GDEPT. Curr. Pharm. Des. 2002;8(15):134961.

147. McNeish I., Searle P., Young L., Kerr D. Gene directed enzyme prodrug therapy for cancer. Advanced Drug Delivery Reviews. 1997 Jul 7;26(2-3):173-84.

148. Holloway BW. Genetic recombination in Pseudomonas aeruginosa. J. Gen. Microbiol. 1955 Dec;13(3):572-81.

149. Hoang TT, Karkhoff-Schweizer RR, Kutchma AJ, Schweizer HP. A broad-hostrange Flp-FRT recombination system for site-specific excision of chromosomallylocated DNA sequences: application for isolation of unmarked Pseudomonas aeruginosa mutants. Gene. 1998;212(1):77-86.

150. Milton DL, O'Toole R, Horstedt P, Wolf-Watz H. Flagellin A is essential for the virulence of Vibrio anguillarum. J. Bacteriol. 1996 Mar;178(5):1310-9.

151. Owen JG, Ackerley DF. Characterization of pyoverdine and achromobactin in Pseudomonas syringae pv. phaseolicola 1448a. BMC Microbiol. 2011 Oct 3;11:218.

152. Altschul SF, Gish W, Miller W, Myers EW, Lipman DJ. Basic local alignment search tool. J. Mol. Biol. 1990 Oct 5;215(3):403-10.

153. Winsor GL, Lam DKW, Fleming L, Lo R, Whiteside MD, Yu NY, et al. Pseudomonas Genome Database: improved comparative analysis and population genomics capability for Pseudomonas genomes. Nucleic Acids Res. 2011 Jan;39(Database issue):D596-600.

154. Larkin MA, Blackshields G, Brown NP, Chenna R, McGettigan PA, McWilliam H, et al. Clustal W and Clustal X version 2.0. Bioinformatics. 2007 Nov 1;23(21):29478. 
155. Zhao Y, Simon R. BRB-ArrayTools Data Archive for Human Cancer Gene Expression: A Unique and Efficient Data Sharing Resource. Cancer Inform. 6:915.

156. Huang DW, Sherman BT, Lempicki RA. Systematic and integrative analysis of large gene lists using DAVID bioinformatics resources. Nat Protoc. 2009;4(1):4457.

157. GeneChip P. aeruginosa Genome Array [Internet]. Available from: http://www.affymetrix.com/browse/products.jsp?productld=131484\&navMode $=34000 \&$ navAction=jump\&ald=productsNav\#1_1

158. Klebanoff SJ. Oxygen Metabolism and the Toxic Properties of Phagocytes. Ann Intern Med. 1980 Sep 1;93(3):480-9.

159. Fielden EM, Roberts PB, Bray RC, Lowe DJ, Mautner GN, Rotilio G, et al. The mechanism of action of superoxide dismutase from pulse radiolysis and electron paramagnetic resonance. Evidence that only half the active sites function in catalysis. Biochem J. 1974 Apr;139(1):49-60.

160. Dolman D, Newell GA, Thurlow MD. A kinetic study of the reaction of horseradish peroxidase with hydrogen peroxide. Canadian journal of biochemistry. 1975;53(5):495-501.

161. Hassett DJ, Imlay JA. Oxidative Stress Systems in Bacteria: Four Model Organisms. In: Nickerson CA, Schurr MJ, editors. Molecular Paradigms of Infectious Disease [Internet]. Springer US; 2006 [cited 2012 Jun 3]. p. 544-74. Available from: http://www.springerlink.com/content/w3t2512p25777286/abstract/

162. Ardissone $S$, Frendo $P$, Laurenti E, Jantschko W, Obinger C, Puppo A, et al. Purification and Physical-Chemical Characterization of the Three Hydroperoxidases from the Symbiotic Bacterium Sinorhizobium melilotit. Biochemistry. 2004;43(39):12692-9.

163. Cohen G, Hochstein P. Glutathione Peroxidase: The Primary Agent for the Elimination of Hydrogen Peroxide in Erythrocytes*. Biochemistry. 1963;2(6):1420-8.

164. Nakamura J, Purvis ER, Swenberg JA. Micromolar concentrations of hydrogen peroxide induce oxidative DNA lesions more efficiently than millimolar concentrations in mammalian cells. Nucleic Acids Res. 2003 Mar 15;31(6):17905 .

165. Goor G, Glenneberg J, Jacobi S. Hydrogen Peroxide. Ullmann's Encyclopedia of Industrial Chemistry [Internet]. Wiley-VCH Verlag GmbH \& Co. KGaA; 2000 [cited 2012 Jun 27]. Available from: http://onlinelibrary.wiley.com/doi/10.1002/14356007.a13_443.pub2/abstract

166. Behishti N, McIntosh A. A Biometric Study of the Explosive Discharge of the Bombardier Beetle. International Journal of Design and Nature. 2006;1(1):1-9. 
167. Monks TJ, Lau SS. The Pharmacology and Toxicology of Polyphenolic-Glutathione Conjugates. Annual Review of Pharmacology and Toxicology. 1998;38(1):229-55.

168. Zenno S, Koike H, Tanokura M, Saigo K. Gene cloning, purification, and characterization of $\mathrm{NfsB}$, a minor oxygen-insensitive nitroreductase from Escherichia coli, similar in biochemical properties to FRase I, the major flavin reductase in Vibrio fischeri. J. Biochem. 1996 Oct;120(4):736-44.

169. Rau J, Stolz A. Oxygen-insensitive nitroreductases NfsA and NfsB of Escherichia coli function under anaerobic conditions as lawsone-dependent Azo reductases. Appl. Environ. Microbiol. 2003 Jun;69(6):3448-55.

170. Hong Y, Wang G, Maier RJ. The NADPH quinone reductase MdaB confers oxidative stress resistance to Helicobacter hepaticus. Microb. Pathog. 2008 Feb;44(2):169-74.

171. Gorman J, Shapiro L. Crystal structures of the tryptophan repressor binding protein WrbA and complexes with flavin mononucleotide. Protein Science. 2005;14(12):3004-12.

172. Grandori R, Khalifah P, Boice JA, Fairman R, Giovanielli K, Carey J. Biochemical characterization of WrbA, founding member of a new family of multimeric flavodoxin-like proteins. J. Biol. Chem. 1998 Aug 14;273(33):20960-6.

173. Chang D-E, Smalley DJ, Conway T. Gene expression profiling of Escherichia coli growth transitions: an expanded stringent response model. Mol. Microbiol. 2002 Jul;45(2):289-306.

174. PA14 Transposon Insertion Mutant Library [Internet]. Available from: http://ausubellab.mgh.harvard.edu/cgi-bin/pa14/home.cgi

175. Electron-Transport Chains and Their Proton Pumps - Molecular Biology of the Cell - NCBI Bookshelf [Internet]. [cited 2012 Jun 30]. Available from: http://www.ncbi.nlm.nih.gov/books/NBK26904/

176. Eriksson S, Lucchini S, Thompson A, Rhen M, Hinton JC. Unravelling the biology of macrophage infection by gene expression profiling of intracellular Salmonella enterica. Mol Microbiol. 2003;47(1):103-18.

177. Covert J, Eskra L, Splitter G. Isolation of Brucella abortus total RNA from B. abortus-infected murine RAW macrophages. J Microbiol Methods. 2005;60(3):383-93.

178. Srivastava V, Rouanet C, Srivastava R, Ramalingam B, Locht C, Srivastava BS. Macrophage-specific Mycobacterium tuberculosis genes: identification by green fluorescent protein and kanamycin resistance selection. Microbiology. 2007;153(Pt 3):659-66.

179. Dietrich G, Schaible UE, DiehI KD, Mollenkopf H, Wiek S, Hess J, et al. Isolation of RNA from mycobacteria grown under in vitro and in vivo conditions. FEMS Microbiol Lett. 2000;186(2):177-80. 
180. Lynch MD, Gill RT, Stephanopoulos G. Mapping phenotypic landscapes using DNA micro-arrays. Metab. Eng. 2004 Jul;6(3):177-85.

181. GeneChip ${ }^{\circledR}$ Microarray Curriculum Activity \#2 - Structure \& Function of GeneChip Microarrays [Internet]. www.affymetrix.com. 2005. Available from: http://media.affymetrix.com/about_affymetrix/outreach/lesson_plan/downloa ds/student_manual_activities/activity2/activity2_structure_function.pdf

182. Benfey PN, Protopapas AD. Genomics. Prentice-Hall; 2005.

183. Choe SE, Boutros M, Michelson AM, Church GM, Halfon MS. Preferred analysis methods for Affymetrix GeneChips revealed by a wholly defined control dataset. Genome Biology. 2005;6(2):R16.

184. Li C, Wong WH. Model-Based Analysis of Oligonucleotide Arrays: Expression Index Computation and Outlier Detection. PNAS. 2001 Feb 1;98(1):31-6.

185. Trevino V, Falciani F, Barrera-Saldaña HA. DNA Microarrays: a Powerful Genomic Tool for Biomedical and Clinical Research. Mol Med. 2007;13(9-10):527-41.

186. Leiske DL, Karimpour-Fard A, Hume PS, Fairbanks BD, Gill RT. A comparison of alternative 60-mer probe designs in an in-situ synthesized oligonucleotide microarray. BMC Genomics. 2006;7:72.

187. Peeters JK, Van der Spek PJ. Growing applications and advancements in microarray technology and analysis tools. Cell Biochem. Biophys. 2005;43(1):149-66.

188. Lewin B. The Messenger RNA Template. Oxford: Oxford University Press; 1990.

189. Deutscher MP. Degradation of RNA in bacteria: comparison of mRNA and stable RNA. Nucleic Acids Res. 2006;34(2):659-66.

190. Baker KE, Condon C. Under the Tucson Sun: A Meeting in the Desert on mRNA Decay. RNA. 2004 Jan 11;10(11):1680-91.

191. Mangan JA, Sole KM, Mitchison DA, Butcher PD. An Effective Method of RNA Extraction from Bacteria Refractory to Disruption, Including Mycobacteria. Nucl. Acids Res. 1997 Jan 2;25(3):675-6.

192. Deutscher MP. Degradation of Stable RNA in Bacteria. J. Biol. Chem. 2003 Nov $14 ; 278(46): 45041-4$.

193. Levy SB. Very stable prokaryotic messenger RNA in chromosomeless Escherichia coli minicells. Proceedings of the National Academy of Sciences. 1975;72:29004.

194. Alifano P, Bruni CB, Carlomagno MS. Control of mRNA processing and decay in prokaryotes. Genetica. 1994;94(2-3):157-72.

195. Deana A, Belasco JG. Lost in Translation: The Influence of Ribosomes on Bacterial mRNA Decay. Genes Dev. 2005 Jan 11;19(21):2526-33. 
196. Edgar R, Domrachev M, Lash AE. Gene Expression Omnibus: NCBI Gene Expression and Hybridization Array Data Repository. Nucl. Acids Res. 2002 Jan 1;30(1):207-10.

197. Palma M, DeLuca D, Worgall S, Quadri LEN. Transcriptome Analysis of the Response of Pseudomonas aeruginosa to Hydrogen Peroxide. J Bacteriol. 2004 Jan;186(1):248-52.

198. Palma M, Zurita J, Ferreras JA, Worgall S, Larone DH, Shi L, et al. Pseudomonas aeruginosa SoxR does not conform to the archetypal paradigm for SoxRdependent regulation of the bacterial oxidative stress adaptive response. Infect Immun. 2005;73(5):2958-66.

199. Chang W, Small DA, Toghrol F, Bentley WE. Microarray analysis of Pseudomonas aeruginosa reveals induction of pyocin genes in response to hydrogen peroxide. Bmc Genomics. 2005;6:115.

200. Goldová J, Ulrych A, Hercík K, Branny P. A eukaryotic-type signalling system of Pseudomonas aeruginosa contributes to oxidative stress resistance, intracellular survival and virulence. BMC Genomics. 2011;12(1):437.

201. Mackenzie EL, Iwasaki K, Tsuji Y. Intracellular Iron Transport and Storage: From Molecular Mechanisms to Health Implications. Antioxid Redox Signal. 2008 Jun;10(6):997-1030.

202. Dekimpe V, Déziel E. Revisiting the Quorum-Sensing Hierarchy in Pseudomonas Aeruginosa: The Transcriptional Regulator RhIR Regulates LasR-Specific Factors. Microbiology. 2009 Jan 3;155(3):712-23.

203. Frisk A, Schurr JR, Wang G, Bertucci DC, Marrero L, Hwang SH, et al. Transcriptome Analysis of Pseudomonas aeruginosa after Interaction with Human Airway Epithelial Cells. Infect. Immun. 2004 Sep 1;72(9):5433-8.

204. Chugani S, Greenberg EP. The Influence of Human Respiratory Epithelia on Pseudomonas aeruginosa Gene Expression. Microb Pathog. 2007 Jan;42(1):2935.

205. Alhede $M$, Bjarnsholt T, Jensen P $\varnothing$, Phipps RK, Moser C, Christophersen L, et al. Pseudomonas aeruginosa recognizes and responds aggressively to the presence of polymorphonuclear leukocytes. Microbiology. 2009 Nov 1;155(11):3500 3508.

206. Di Carlo E, Forni G, Lollini P, Colombo MP, Modesti A, Musiani P. The Intriguing Role of Polymorphonuclear Neutrophils in Antitumor Reactions. Blood. 2001 Jan 15;97(2):339-45.

207. Reynolds HY. Lung Inflammation: Normal Host Defense or a Complication of Some Diseases? Annual Review of Medicine. 1987;38(1):295-323.

208. Nelson DJ, McMenamin C, McWilliam AS, Brenan M, Holt PG. Development of the airway intraepithelial dendritic cell network in the rat from class II major 
histocompatibility (la)-negative precursors: differential regulation of la expression at different levels of the respiratory tract. J. Exp. Med. 1994 Jan 1;179(1):203-12.

209. Nathan C, Shiloh MU. Reactive oxygen and nitrogen intermediates in the relationship between mammalian hosts and microbial pathogens. Proc Natl Acad Sci U S A. 2000 Aug 1;97(16):8841-8.

210. Hampton MB, Kettle AJ, Winterbourn CC. Inside the Neutrophil Phagosome: Oxidants, Myeloperoxidase, and Bacterial Killing. Blood. 1998 Nov 1;92(9):300717.

211. Nauseef WM. How human neutrophils kill and degrade microbes: an integrated view. Immunological Reviews. 2007 Oct 1;219(1):88-102.

212. Rosenberger CM, Finlay BB. Phagocyte sabotage: disruption of macrophage signalling by bacterial pathogens. Nat Rev Mol Cell Biol. 2003;4(5):385-96.

213. Fux CA, Shirtliff M, Stoodley P, Costerton JW. Can laboratory reference strains mirror 'real-world' pathogenesis? Trends Microbiol. 2005;13(2):58-63.

214. Morrow DM, Entezari-Zaher T, Romashko J, Azghani AO, Javdan M, Ulloa L, et al. Antioxidants preserve macrophage phagocytosis of Pseudomonas aeruginosa during hyperoxia. Free Radic Biol Med. 2007;42(9):1338-49.

215. Small DA, Chang W, Toghrol F, Bentley WE. Toxicogenomic analysis of sodium hypochlorite antimicrobial mechanisms in Pseudomonas aeruginosa. Appl Microbiol Biotechnol. 2007;74(1):176-85.

216. Stewart PS, Franklin MJ. Physiological heterogeneity in biofilms. Nature Reviews Microbiology. 2008 Mar 1;6(3):199-210.

217. Berney M, Hammes F, Bosshard F, Weilenmann H-U, Egli T. Assessment and interpretation of bacterial viability by using the LIVE/DEAD BacLight Kit in combination with flow cytometry. Appl. Environ. Microbiol. 2007 May;73(10):3283-90.

218. Biggerstaff JP, Le Puil M, Weidow BL, Prater J, Glass K, Radosevich M, et al. New methodology for viability testing in environmental samples. Mol. Cell. Probes. 2006 Apr;20(2):141-6.

219. Stocks SM. Mechanism and use of the commercially available viability stain, BacLight. Cytometry A. 2004 Oct;61(2):189-95.

220. Fiedler SL, Izvekov S, Violi A. The effect of temperature on nanoparticle clustering. Carbon. 2007 Aug;45(9):1786-94.

221. Li YM, Mitsuhashi T, Wojciechowicz D, Shimizu N, Li J, Stitt A, et al. Molecular identity and cellular distribution of advanced glycation endproduct receptors: relationship of p60 to OST-48 and p90 to $80 \mathrm{~K}-\mathrm{H}$ membrane proteins. Proc. Natl. Acad. Sci. U.S.A. 1996 Oct 1;93(20):11047-52. 
222. Ralph P, Nakoinz I. Antibody-dependent killing of erythrocyte and tumor targets by macrophage-related cell lines: enhancement by PPD and LPS. J. Immunol. 1977 Sep;119(3):950-4.

223. Raschke WC, Baird S, Ralph P, Nakoinz I. Functional macrophage cell lines transformed by Abelson leukemia virus. Cell. 1978 Sep;15(1):261-7.

224. Di A, Brown ME, Deriy LV, Li C, Szeto FL, Chen Y, et al. CFTR regulates phagosome acidification in macrophages and alters bactericidal activity. Nature Cell Biology. 2006;8(9):933-44.

225. Lamothe J, Valvano MA. Burkholderia cenocepacia-induced delay of acidification and phagolysosomal fusion in cystic fibrosis transmembrane conductance regulator (CFTR)-defective macrophages. Microbiology. 2008 Jan 12;154(12):3825-34.

226. Porto PD, Cifani N, Guarnieri S, Di Domenico EG, Mariggiò MA, Spadaro F, et al. Dysfunctional CFTR Alters the Bactericidal Activity of Human Macrophages against Pseudomonas aeruginosa. PLoS ONE. 2011 May 18;6(5):e19970.

227. Vieira OV, Botelho RJ, Grinstein S. Phagosome maturation: aging gracefully. Biochem J. 2002 Sep 15;366(Pt 3):689-704.

228. Haas A. The Phagosome: Compartment with a License to Kill. Traffic. 2007;8(4):311-30.

229. Schroeder A, Mueller O, Stocker S, Salowsky R, Leiber M, Gassmann M, et al. The RIN: an RNA integrity number for assigning integrity values to RNA measurements. BMC Mol Biol. 2006 Jan 31;7:3.

230. Smyth GK. Linear models and empirical bayes methods for assessing differential expression in microarray experiments. Stat Appl Genet Mol Biol. 2004;3:Article3.

231. Gentleman RC, Carey VJ, Bates DM, Bolstad B, Dettling M, Dudoit S, et al. Bioconductor: open software development for computational biology and bioinformatics. Genome Biol. 2004;5(10):R80.

232. Brent MR, Guigó R. Recent advances in gene structure prediction. Curr. Opin. Struct. Biol. 2004 Jun;14(3):264-72.

233. Sridhar J, Sabarinathan R, Balan SS, Rafi ZA, Gunasekaran P, Sekar K. Junker: An Intergenic Explorer for Bacterial Genomes. Genomics, Proteomics \& Bioinformatics. 2011 Oct;9(4-5):179-82.

234. Fu LM. Functional Analysis of Intergenic Regions for Gene Discovery. In: Lopes H, editor. Computational Biology and Applied Bioinformatics [Internet]. InTech; 2011 [cited 2012 May 24]. Available from: http://www.intechopen.com/books/computational-biology-and-appliedbioinformatics/functional-analysis-of-intergenic-regions-for-gene-discovery 
235. Plague GR. Intergenic Transposable Elements Are Not Randomly Distributed in Bacteria. Genome Biol Evol. 2010 Jan 1;2:584-90.

236. Cooper VS, Schneider D, Blot M, Lenski RE. Mechanisms Causing Rapid and Parallel Losses of Ribose Catabolism in Evolving Populations of Escherichia Coli B. J. Bacteriol. 2001 Jan 5;183(9):2834-41.

237. Huang DW, Sherman BT, Lempicki RA. Bioinformatics enrichment tools: paths toward the comprehensive functional analysis of large gene lists. Nucleic Acids Res. 2009 Jan;37(1):1-13.

238. Benjamini Y, Hochberg Y. Controlling the False Discovery Rate: A Practical and Powerful Approach to Multiple Testing. Journal of the Royal Statistical Society. Series B (Methodological). 1995 Jan 1;57(1):289-300.

239. Mao F, Dam P, Chou J, Olman V, Xu Y. DOOR: a database for prokaryotic operons. Nucleic Acids Research. 2009 Jan 1;37(Database):D459-D463.

240. Galán-Vásquez E, Luna B, Martínez-Antonio A. The Regulatory Network of Pseudomonas aeruginosa. Microbial Informatics and Experimentation. 2011 Jun 14;1(1):3.

241. al-Sarireh B, Eremin O. Tumour-associated macrophages (TAMS): disordered function, immune suppression and progressive tumour growth. J R Coll Surg Edinb. 2000 Feb;45(1):1-16.

242. Parret AH., De Mot R. Bacteria killing their own kind: novel bacteriocins of Pseudomonas and other $\mathrm{p}$-proteobacteria. Trends in Microbiology. $2002 \mathrm{Mar}$ 1;10(3):107-12.

243. Michel-Briand $Y$, Baysse $C$. The pyocins of Pseudomonas aeruginosa. Biochimie. 2002;84(5-6):499-510.

244. Nakayama K, Takashima K, Ishihara H, Shinomiya T, Kageyama M, Kanaya S, et al. The R-type pyocin of Pseudomonas aeruginosa is related to P2 phage, and the F-type is related to lambda phage. Mol. Microbiol. 2000 Oct;38(2):213-31.

245. Huston WM, Potter AJ, Jennings MP, Rello J, Hauser AR, McEwan AG. Survey of Ferroxidase Expression and Siderophore Production in Clinical Isolates of Pseudomonas aeruginosa. J Clin Microbiol. 2004 Jun;42(6):2806-9.

246. Cox CD, Adams P. Siderophore Activity of Pyoverdin for Pseudomonas Aeruginosa. Infect. Immun. 1985 Jan 4;48(1):130-8.

247. Meyer JM, Neely A, Stintzi A, Georges C, Holder IA. Pyoverdin is essential for virulence of Pseudomonas aeruginosa. Infect. Immun. 1996 Feb;64(2):518-23.

248. Ankenbauer R, Sriyosachati S, Cox CD. Effects of Siderophores on the Growth of Pseudomonas Aeruginosa in Human Serum and Transferrin. Infect. Immun. 1985 Jan 7;49(1):132-40. 
249. Sriyosachati $S$, Cox CD. Siderophore-Mediated Iron Acquisition from Transferrin by Pseudomonas Aeruginosa. Infect. Immun. 1986 Jan 6;52(3):885-91.

250. Schmidt-Ott KM, Mori K, Li JY, Kalandadze A, Cohen DJ, Devarajan P, et al. Dual Action of Neutrophil Gelatinase-Associated Lipocalin. JASN. 2007 Jan 2;18(2):407-13.

251. Miethke M, Marahiel MA. Siderophore-based iron acquisition and pathogen control. Microbiol. Mol. Biol. Rev. 2007 Sep;71(3):413-51.

252. Gruenheid S, Canonne-Hergaux F, Gauthier S, Hackam DJ, Grinstein S, Gros P. The iron transport protein NRAMP2 is an integral membrane glycoprotein that colocalizes with transferrin in recycling endosomes. J. Exp. Med. 1999 Mar 1;189(5):831-41.

253. Henle ES, Linn S. Formation, prevention, and repair of DNA damage by iron/hydrogen peroxide. J. Biol. Chem. 1997 Aug 1;272(31):19095-8.

254. Kuhn DE, Lafuse WP, Zwilling BS. Iron Transport into Mycobacterium AviumContaining Phagosomes from an Nramp1Gly169-Transfected RAW264.7 Macrophage Cell Line. J Leukoc Biol. 2001 Jan 1;69(1):43-9.

255. Wagner D, Maser J, Moric I, Boechat N, Vogt S, Gicquel B, et al. Changes of the Phagosomal Elemental Concentrations by Mycobacterium Tuberculosis Mramp. Microbiology. 2005 Jan 1;151(1):323-32.

256. Zhong W, Lafuse WP, Zwilling BS. Infection with Mycobacterium aviumDifferentially Regulates the Expression of Iron Transport Protein mRNA in Murine Peritoneal Macrophages. Infect. Immun. 2001 Jan 11;69(11):6618-24.

257. Wooldridge KG, Williams PH. Iron uptake mechanisms of pathogenic bacteria. FEMS Microbiology Reviews. 1993;12(4):325-48.

258. Xiao R, Kisaalita WS. Iron acquisition from transferrin and lactoferrin by Pseudomonas aeruginosa pyoverdin. Microbiology (Reading, Engl.). 1997 Jul;143 ( Pt 7):2509-15.

259. Wolz C, Hohloch K, Ocaktan A, Poole K, Evans RW, Rochel N, et al. Iron release from transferrin by pyoverdin and elastase from Pseudomonas aeruginosa. Infect. Immun. 1994 Sep;62(9):4021-7.

260. Takase H, Nitanai H, Hoshino K, Otani T. Impact of Siderophore Production onPseudomonas Aeruginosa Infections in Immunosuppressed Mice. Infect. Immun. 2000 Jan 4;68(4):1834-9.

261. Michel L, González N, Jagdeep S, Nguyen-Ngoc T, Reimmann C. PchR-box recognition by the AraC-type regulator PchR of Pseudomonas aeruginosa requires the siderophore pyochelin as an effector. Mol. Microbiol. 2005 Oct;58(2):495-509. 
262. Britigan BE, Rasmussen GT, Cox CD. Pseudomonas siderophore pyochelin enhances neutrophil-mediated endothelial cell injury. AM.J.PHYSIOL. 1994;266(2 part 1):L192-L198.

263. Britigan BE, Roeder TL, Rasmussen GT, Shasby DM, McCormick ML, Cox CD. Interaction of the Pseudomonas aeruginosa secretory products pyocyanin and pyochelin generates hydroxyl radical and causes synergistic damage to endothelial cells. Implications for Pseudomonas-associated tissue injury. Journal of Clinical Investigation. 1992;90(6):2187-96.

264. Britigan BE, Rasmussen GT, Cox CD. Binding of Iron and Inhibition of IronDependent Oxidative Cell Injury by the 'Calcium Chelator' 1,2-Bis(2Aminophenoxy)Ethane $\mathrm{N}, \mathrm{N}, \mathrm{N}^{\prime}, \mathrm{N}^{\prime}$-tetraacetic Acid (BAPTA). Biochemical Pharmacology. 1998 Feb 1;55(3):287-95.

265. Braud A, Hoegy F, Jezequel K, Lebeau T, Schalk IJ. New insights into the metal specificity of the Pseudomonas aeruginosa pyoverdine-iron uptake pathway. Environ. Microbiol. 2009 May;11(5):1079-91.

266. Schalk IJ, Hannauer M, Braud A. New roles for bacterial siderophores in metal transport and tolerance. Environmental Microbiology. 2011;13(11):2844-54.

267. Matthijs S, Tehrani KA, Laus G, Jackson RW, Cooper RM, Cornelis P. Thioquinolobactin, a Pseudomonas siderophore with antifungal and antiPythium activity. Environ. Microbiol. 2007 Feb;9(2):425-34.

268. Gallagher LA, Manoil C. Pseudomonas aeruginosa PAO1 Kills Caenorhabditis elegans by Cyanide Poisoning. J Bacteriol. 2001 Nov;183(21):6207-14.

269. Ryan RW, Tilton RC. The isolation of rhodanese from Pseudomonas aeruginosa by affinity chromatography. J. Gen. Microbiol. 1977 Nov;103(1):197-9.

270. Solomonson L. Cyanide as a metabolic inhibitor. Cyanide Biology. London; 1981. p. 11-28.

271. Cipollone R, Frangipani E, Tiburzi F, Imperi F, Ascenzi P, Visca P. Involvement of Pseudomonas Aeruginosa Rhodanese in Protection from Cyanide Toxicity. Appl. Environ. Microbiol. 2007 Jan 15;73(2):390-8.

272. Cipollone R, Ascenzi P, Frangipani E, Visca P. Cyanide detoxification by recombinant bacterial rhodanese. Chemosphere. 2006 May;63(6):942-9.

273. Carterson AJ, Morici LA, Jackson DW, Frisk A, Lizewski SE, Jupiter R, et al. The transcriptional regulator AlgR controls cyanide production in Pseudomonas aeruginosa. J. Bacteriol. 2004 Oct;186(20):6837-44.

274. Pessi G, Haas D. Transcriptional Control of the Hydrogen Cyanide Biosynthetic Genes hcnABC by the Anaerobic Regulator ANR and the Quorum-Sensing Regulators LasR and RhIR inPseudomonas Aeruginosa. J. Bacteriol. 2000 Dec 15;182(24):6940-9. 
275. Girard G, Bloemberg GV. Central role of quorum sensing in regulating the production of pathogenicity factors in Pseudomonas aeruginosa. Future Microbiol. 2008 Feb;3(1):97-106.

276. Schuster M, Lostroh CP, Ogi T, Greenberg EP. Identification, Timing, and Signal Specificity of Pseudomonas aeruginosa Quorum-Controlled Genes: a Transcriptome Analysis. J Bacteriol. 2003 Apr;185(7):2066-79.

277. Strieker M, Tanović A, Marahiel MA. Nonribosomal peptide synthetases: structures and dynamics. Curr. Opin. Struct. Biol. 2010 Apr;20(2):234-40.

278. Fan Y. Quorum Sensing Signal Interference within and across the Kingdoms [Internet] [Doctoral]. [Singapore]: National University of Singapore; 2008. Available from: http://scholarbank.nus.edu.sg/bitstream/handle/10635/12886/yfthesis2008.pdf?sequence $=1$

279. Turner KH, Vallet-Gely I, Dove SL. Epigenetic Control of Virulence Gene Expression in Pseudomonas aeruginosa by a LysR-Type Transcription Regulator. PLoS Genet. 2009 Dec 18;5(12):e1000779.

280. Rodriguez-Rojas A, Blazquez J. The Pseudomonas aeruginosa pfpl Gene Plays an Antimutator Role and Provides General Stress Protection. J. Bacteriol. 2009 Feb 1;191(3):844-50.

281. Nguyen D, Singh PK. Evolving stealth: genetic adaptation of Pseudomonas aeruginosa during cystic fibrosis infections. Proc. Natl. Acad. Sci. U.S.A. 2006 May 30;103(22):8305-6.

282. Hogardt M, Roeder M, Schreff AM, Eberl L, Heesemann J. Expression of Pseudomonas aeruginosa exoS is controlled by quorum sensing and RpoS. Microbiology (Reading, Engl.). 2004 Apr;150(Pt 4):843-51.

283. Maciá MD, Blanquer D, Togores B, Sauleda J, Pérez JL, Oliver A. Hypermutation Is a Key Factor in Development of Multiple-Antimicrobial Resistance in Pseudomonas Aeruginosa Strains Causing Chronic Lung Infections. Antimicrob. Agents Chemother. 2005 Jan 8;49(8):3382-6.

284. Oliver A, Levin BR, Juan C, Baquero F, Blazquez J. Hypermutation and the preexistence of antibiotic-resistant Pseudomonas aeruginosa mutants: Implications for susceptibility testing and treatment of chronic infections. Antimicrobial Agents and Chemotherapy. 2004;48(11):4226-33.

285. Bianchi SM, Prince LR, McPhillips K, Allen L, Marriott HM, Taylor GW, et al. Impairment of apoptotic cell engulfment by pyocyanin, a toxic metabolite of Pseudomonas aeruginosa. Am. J. Respir. Crit. Care Med. 2008 Jan 1;177(1):3543.

286. DeLeo FR. Modulation of phagocyte apoptosis by bacterial pathogens. Apoptosis. 2004 Jul;9(4):399-413. 
287. Monick MM, Powers LS, Barrett CW, Hinde S, Ashare A, Groskreutz DJ, et al. Constitutive ERK MAPK activity regulates macrophage ATP production and mitochondrial integrity. J. Immunol. 2008 Jun 1;180(11):7485-96.

288. Zhang Y, Li X, Carpinteiro A, Gulbins E. Acid sphingomyelinase amplifies redox signaling in Pseudomonas aeruginosa-induced macrophage apoptosis. J Immunol. 2008;181(6):4247-54.

289. Schmitz C, Axmacher B, Zunker U, Korr H. Age-related changes of DNA repair and mitochondrial DNA synthesis in the mouse brain. Acta Neuropathologica. 1999;97(1):71-81.

290. Slater AF, Nobel CS, Orrenius S. The role of intracellular oxidants in apoptosis. Biochim. Biophys. Acta. 1995 May 24;1271(1):59-62.

291. Glauser MP, Zanetti G, Baumgartner JD, Cohen J. Septic shock: pathogenesis. Lancet. 1991 Sep 21;338(8769):732-6.

292. Tsai WC, Strieter RM, Mehrad B, Newstead MW, Zeng X, Standiford TJ. CXC Chemokine Receptor CXCR2 Is Essential for Protective Innate Host Response in Murine Pseudomonas aeruginosa Pneumonia. Infect Immun. 2000 Jul;68(7):4289-96.

293. Friedman L, Kolter R. Two Genetic Loci Produce Distinct Carbohydrate-Rich Structural Components of the Pseudomonas Aeruginosa Biofilm Matrix. J. Bacteriol. 2004 Jul 15;186(14):4457-65.

294. Jackson KD, Starkey M, Kremer S, Parsek MR, Wozniak DJ. Identification of Psl, a Locus Encoding a Potential Exopolysaccharide That Is Essential for Pseudomonas Aeruginosa PAO1 Biofilm Formation. J. Bacteriol. 2004 Jul 15;186(14):4466-75.

295. Matsukawa M, Greenberg EP. Putative Exopolysaccharide Synthesis Genes Influence Pseudomonas Aeruginosa Biofilm Development. J. Bacteriol. 2004 Jul 15;186(14):4449-56.

296. Haslett C. Granulocyte apoptosis and its role in the resolution and control of lung inflammation. Am. J. Respir. Crit. Care Med. 1999 Nov;160(5 Pt 2):S5-11.

297. Mahajan-Miklos S, Tan M-W, Rahme LG, Ausubel FM. Molecular Mechanisms of Bacterial Virulence Elucidated Using a Pseudomonas aeruginosa-Caenorhabditis elegans Pathogenesis Model. Cell. 1999 Jan 8;96(1):47-56.

298. Mai GT, Seow WK, Pier GB, McCormack JG, Thong YH. Suppression of lymphocyte and neutrophil functions by Pseudomonas aeruginosa mucoid exopolysaccharide (alginate): reversal by physicochemical, alginase, and specific monoclonal antibody treatments. Infect Immun. 1993 Feb;61(2):559-64.

299. Speert DP, Wright SD, Silverstein SC, Mah B. Functional characterization of macrophage receptors for in vitro phagocytosis of unopsonized Pseudomonas aeruginosa. Journal of Clinical Investigation. 1988 Sep 1;82(3):872-9. 
300. Byrd MS, Sadovskaya I, Vinogradov E, Lu H, Sprinkle AB, Richardson SH, et al. Genetic and biochemical analyses of the Pseudomonas aeruginosa Psl exopolysaccharide reveal overlapping roles for polysaccharide synthesis enzymes in Psl and LPS production. Mol. Microbiol. 2009 Aug;73(4):622-38.

301. Fuqua WC, Winans SC, Greenberg EP. Quorum sensing in bacteria: the LuxR-LuxI family of cell density-responsive transcriptional regulators. J Bacteriol. 1994 Jan;176(2):269-75.

302. Dachs GU, Hunt MA, Syddall S, Singleton DC, Patterson AV. Bystander or No Bystander for Gene Directed Enzyme Prodrug Therapy. Molecules. 2009 Nov 10;14(11):4517-45.

303. Portsmouth D, Hlavaty J, Renner M. Suicide genes for cancer therapy. Mol. Aspects Med. 2007 Feb;28(1):4-41.

304. Gadi VK, Alexander SD, Kudlow JE, Allan P, Parker WB, Sorscher EJ. In vivo sensitization of ovarian tumors to chemotherapy by expression of E. coli purine nucleoside phosphorylase in a small fraction of cells. Gene Ther. 2000 Oct;7(20):1738-43.

305. Rainov NG. A phase III clinical evaluation of herpes simplex virus type 1 thymidine kinase and ganciclovir gene therapy as an adjuvant to surgical resection and radiation in adults with previously untreated glioblastoma multiforme. Hum. Gene Ther. 2000 Nov 20;11(17):2389-401.

306. Freytag SO, Khil M, Stricker H, Peabody J, Menon M, DePeralta-Venturina M, et al. Phase I study of replication-competent adenovirus-mediated double suicide gene therapy for the treatment of locally recurrent prostate cancer. Cancer Res. 2002 Sep 1;62(17):4968-76.

307. Patterson AV, Saunders MP, Greco O. Prodrugs in genetic chemoradiotherapy. Curr. Pharm. Des. 2003;9(26):2131-54.

308. Tanaka T, Duflot-Dancer A, Tiraby M, Piccoli C, Tiraby G, Yamasaki H, et al. Bystander effect from cytosine deaminase and uracil phosphoribosyl transferase genes in vitro: a partial contribution of gap junctions. Cancer Lett. 2009 Sep 8;282(1):43-7.

309. Chung-Faye G, Palmer D, Anderson D, Clark J, Downes M, Baddeley J, et al. Virusdirected, enzyme prodrug therapy with nitroimidazole reductase: a phase I and pharmacokinetic study of its prodrug, CB1954. Clin. Cancer Res. 2001 Sep;7(9):2662-8.

310. Patel P, Young JG, Mautner V, Ashdown D, Bonney S, Pineda RG, et al. A phase I/II clinical trial in localized prostate cancer of an adenovirus expressing nitroreductase with CB1954 [correction of CB1984]. Mol. Ther. 2009 Jul;17(7):1292-9. 
311. Mitchell DJ, Minchin RF. E. coli nitroreductase/CB1954 gene-directed enzyme prodrug therapy: role of arylamine N-acetlytransferase 2. Cancer Gene Ther. 2008 Nov;15(11):758-64.

312. Prosser GA, Patterson AV, Ackerley DF. uvrB gene deletion enhances SOS chromotest sensitivity for nitroreductases that preferentially generate the 4hydroxylamine metabolite of the anti-cancer prodrug CB1954. Journal of Biotechnology. 2010 Oct 1;150(1):190-4.

313. Swe P, Copp J, Green L, Guise C, Mowday A, Smaill J, et al. Targeted Mutagenesis of the Vibrio fischeri Flavin Reductase FRase I to Improve Activation of the Anticancer Prodrug CB1954. Biochemical Pharmacology. (In Press).

314. Wilson WR, Hicks KO, Pullen SM, Ferry DM, Helsby NA, Patterson AV. Bystander effects of bioreductive drugs: potential for exploiting pathological tumor hypoxia with dinitrobenzamide mustards. Radiat. Res. 2007 Jun;167(6):625-36.

315. Boger DL, Zhou J, Cai H. Demonstration and definition of the noncovalent binding selectivity of agents related to CC-1065 by an affinity cleavage agent: noncovalent binding coincidental with alkylation. Bioorg. Med. Chem. 1996 Jun;4(6):859-67.

316. Gunz D, Hess MT, Naegeli H. Recognition of DNA adducts by human nucleotide excision repair. Evidence for a thermodynamic probing mechanism. J. Biol. Chem. 1996 Oct 11;271(41):25089-98.

317. Wilson WR, Stribbling SM, Pruijn FB, Syddall SP, Patterson AV, Liyanage HDS, et al. Nitro-chloromethylbenzindolines: hypoxia-activated prodrugs of potent adenine N3 DNA minor groove alkylators. Mol Cancer Ther. 2009 Oct 1;8(10):2903-13.

318. Syddall SP. Novel Bacterial Nitroreductases and Bioreductive Substrates for Gene Therapy Applications. [New Zealand]: University of Auckland; 2009.

319. Koronakis V, Eswaran J, Hughes C. Structure and function of TolC: the bacterial exit duct for proteins and drugs. Annu. Rev. Biochem. 2004;73:467-89.

320. Davidov $Y$, Rozen R, Smulski DR, Van Dyk TK, Vollmer AC, Elsemore DA, et al. Improved bacterial SOS promoter\&Colon; lux fusions for genotoxicity detection. Mutat. Res. 2000 Mar 3;466(1):97-107.

321. Shapiro E, Baneyx F. Stress-based identification and classification of antibacterial agents: second-generation Escherichia coli reporter strains and optimization of detection. Antimicrob. Agents Chemother. 2002 Aug;46(8):2490-7.

322. Prosser G. Discovery and Optimisation of Bacterial Nitroreductases for use in Anti-Cancer Gene Therapy. [New Zealand]: Victoria Univeristy of Wellington; 2011.

323. Race PR, Lovering AL, White SA, Grove JI, Searle PF, Wrighton CW, et al. Kinetic and Structural Characterisation of Escherichia coli Nitroreductase Mutants 
Showing Improved Efficacy for the Prodrug Substrate CB1954. Journal of Molecular Biology. 2007 Apr 27;368(2):481-92.

324. Wilson WR, Pullen SM, Hogg A, Helsby NA, Hicks KO, Denny WA. Quantitation of bystander effects in nitroreductase suicide gene therapy using threedimensional cell cultures. Cancer Res. 2002 Mar 1;62(5):1425-32.

325. Heo Y-J, Chung I-Y, Cho W-J, Lee B-Y, Kim J-H, Choi K-H, et al. The Major Catalase Gene (katA) of Pseudomonas aeruginosa PA14 Is under both Positive and Negative Control of the Global Transactivator OxyR in Response to Hydrogen Peroxide. J Bacteriol. 2010 Jan;192(2):381-90.

326. Jayaraman R. Hypermutation and stress adaptation in bacteria. J. Genet. 2011 Aug;90(2):383-91.

327. Funchain P, Yeung A, Stewart JL, Lin R, Slupska MM, Miller JH. The consequences of growth of a mutator strain of Escherichia coli as measured by loss of function among multiple gene targets and loss of fitness. Genetics. 2000 Mar;154(3):95970.

328. Zhou L, Lei X-H, Bochner BR, Wanner BL. Phenotype MicroArray Analysis of Escherichia coli K-12 Mutants with Deletions of All Two-Component Systems. J. Bacteriol. 2003 Aug 15;185(16):4956-72.

329. Hart I. Identifying Unusual Infectious Organisms in Immunocompromised Patients. Reprint from European Clinical Laboratory. 2002;

330. Pitulle C, Citron DM, Bochner B, Barbers R, Appleman MD. Novel bacterium isolated from a lung transplant patient with cystic fibrosis. J. Clin. Microbiol. 1999 Dec;37(12):3851-5.

331. Teehan GS, Bahdouch D, Ruthazer R, Balakrishnan VS, Snydman DR, Jaber BL. Iron Storage Indices: Novel Predictors of Bacteremia in Hemodialysis Patients Initiating Intravenous Iron Therapy. Clin Infect Dis. 2004 Apr 15;38(8):1090-4.

332. Miller MJ, Zhu H, Xu Y, Wu C, Walz AJ, Vergne A, et al. Utilization of microbial iron assimilation processes for the development of new antibiotics and inspiration for the design of new anticancer agents. Biometals. 2009 Feb;22(1):61-75.

333. Ferreras JA, Ryu J-S, Di Lello F, Tan DS, Quadri LEN. Small-molecule inhibition of siderophore biosynthesis in Mycobacterium tuberculosis and Yersinia pestis. Nat. Chem. Biol. 2005 Jun;1(1):29-32.

334. Neres J, Labello NP, Somu RV, Boshoff HI, Wilson DJ, Vannada J, et al. Inhibition of siderophore biosynthesis in Mycobacterium tuberculosis with nucleoside bisubstrate analogues: structure-activity relationships of the nucleobase domain of 5'-O-[N-(salicyl)sulfamoyl]adenosine. J. Med. Chem. 2008 Sep 11;51(17):5349-70. 
335. Quadri LEN. Strategic paradigm shifts in the antimicrobial drug discovery process of the 21st century. Infect Disord Drug Targets. 2007 Sep;7(3):230-7.

336. Wencewicz TA, Möllmann U, Long TE, Miller MJ. Is drug release necessary for antimicrobial activity of siderophore-drug conjugates? Syntheses and biological studies of the naturally occurring salmycin 'Trojan Horse' antibiotics and synthetic desferridanoxamine-antibiotic conjugates. Biometals. 2009 Aug;22(4):633-48.

337. Banin E, Lozinski A, Brady KM, Berenshtein E, Butterfield PW, Moshe M, et al. The potential of desferrioxamine-gallium as an anti-Pseudomonas therapeutic agent. PNAS. 2008 Oct 28;105(43):16761-6.

338. Hiraku Y, Sekine A, Nabeshi H, Midorikawa K, Murata M, Kumagai Y, et al. Mechanisms of carcinogenesis induced by a veterinary antimicrobial drug, nitrofurazone, via oxidative DNA damage and cell proliferation. Cancer Letters. 2004;215:141-50.

339. Karamanakos PN. Furazolidone and Serotonin Syndrome: Is there any Association? Clinics. 2008 Aug;63(4):553-4.

340. Muller M. Mode of action of metronidazole on anaerobic bacteria and protozoa. Surgery. 1983;93:165-71.

341. McOsker C, Fitzpatrick P. Nitrofurantoin: mechanism of action and implications for resistance development in common uropathogens. The Journal of antimicrobial chemotherapy. 1994;33(Suppl A):23-30.

342. Ducel G, Fabry J, Nicolle L, editors. WHO | Prevention of hospital-acquired infections: A practical guide. 2002 [cited 2012 May 2]; Available from: http://www.who.int/csr/resources/publications/drugresist/WHO_CDS_CSR_EPH _2002_12/en/

343. Anderson DJ, Kirkland KB, Kaye KS, Thacker PA 2nd, Kanafani ZA, Auten G, et al. Underresourced hospital infection control and prevention programs: penny wise, pound foolish? Infect Control Hosp Epidemiol. 2007 Jul;28(7):767-73.

344. Mintjes-de Groot AJ, van Hassel CA, Kaan JA, Verkooyen RP, Verbrugh HA. Impact of hospital-wide surveillance on hospital-acquired infections in an acutecare hospital in the Netherlands. J. Hosp. Infect. 2000 Sep;46(1):36-42.

345. Bean DC, Krahe D, Wareham DW. Antimicrobial resistance in community and nosocomial Escherichia coli urinary tract isolates, London 2005 - 2006. Annals of Clinical Microbiology and Antimicrobials. 2008 Jun 18;7(1):13.

346. Li Y, Leung P, Yao L, Song QW, Newton E. Antimicrobial effect of surgical masks coated with nanoparticles. Journal of Hospital Infection. 2006 Jan;62(1):58-63.

347. Han S, Yang Y. Antimicrobial activity of wool fabric treated with curcumin. Dyes and Pigments. 2005 Feb;64(2):157-61. 
348. Gupta D, Khare SK, Laha A. Antimicrobial properties of natural dyes against Gram-negative bacteria. Coloration Technology. 2006 Jun 22;120(4):167-71.

349. Liu Z, Wang F, Bai XE. Research Progress of Antibacterial Fiber and Fabric Used in Clothing. Advanced Materials Research. 2011 Sep;332-334:1790-3.

350. Zhang $\mathrm{Y}$, Peng $\mathrm{H}$, Huang $\mathrm{W}$, Zhou $\mathrm{Y}$, Yan D. Facile preparation and characterization of highly antimicrobial colloid Ag or Au nanoparticles. Journal of Colloid and Interface Science. 2008 Sep 15;325(2):371-6. 